UNIVERSIDADE DE SÃO PAULO

FACULDADE DE MEDICINA DE RIBEIRÃO PRETO

DEPARTAMENTO DE MEDICINA SOCIAL

Avaliação de Métodos Estatísticos Aplicados ao Estudo de Testes Diagnósticos na Presença do Viés de Verificação

DAVI CASALE ARAGON 


\section{Avaliação de Métodos Estatísticos Aplicados ao Estudo de Testes Diagnósticos na Presença do Viés de Verificação}

Dissertação apresentada ao Departamento de Medicina Social da Faculdade de Medicina de Ribeirão Preto da Universidade de São Paulo para a obtenção do título de Mestre em Saúde na Comunidade.

Área de Concentração: Saúde na Comunidade

Orientador: Prof. Dr. Edson Zangiacomi Martinez

Ribeirão Preto 


\section{Ficha Catalográfica}

Aragon, Davi Casale

Avaliação de métodos estatísticos aplicados ao estudo de testes diagnósticos na presença do viés de verificação. Ribeirão Preto, 2007. 114 p.:il.; $30 \mathrm{~cm}$

Dissertação de Mestrado apresentada à Faculdade de Medicina de Ribeirão Preto - USP. Área de concentração: Saúde na Comunidade. Orientador: Martinez, Edson Zangiacomi

1. Viés de verificação. 2. Testes diagnósticos. 3. Métodos bayesianos. 


\section{Folha de Aprovação}

Davi Casale Aragon

Avaliação de métodos estatísticos aplicados ao estudo de testes dignósticos na presença do viés de verificação

Dissertação apresentada ao Departamento de Medicina Social da Faculdade de Medicina de Ribeirão Preto da Universidade de São Paulo para a obtenção do título de Mestre em Saúde na Comunidade.

Área de Concentração: Saúde na Comunidade

Aprovado em:

\section{Banca Examinadora}

Prof.(a) Dr.(a):

Instituição: Assinatura:

Prof.(a) Dr.(a):

Instituição: Assinatura:

Prof.(a) Dr.(a):

Instituição: Assinatura: 


\section{Dedicatória}

Aos meus pais, Flávio e Teresinha, com amor e gratidão, por nunca pouparem esforços para que seus filhos alcançassem seus objetivos. 


\section{Agradecimentos}

A Deus, primeiramente, pela presença constante na minha vida.

Aos meus pais, pelos conselhos, apoio e amor incondicional.

Ao meu orientador, Prof. Dr. Edson Zangiacomi Martinez, pelas oportunidades oferecidas e pela dedicação, segurança e competência demonstradas no desenvolvimento deste trabalho.

Ao amigo Edson Zangiacomi Martinez, pela compreensão e ajuda nos meus não raros momentos de instabilidade emocional. Obrigado por ter acreditado em mim.

Ao Prof. Dr. Jorge Alberto Achcar, pelo incentivo e contribuição para o meu crescimento científico.

Aos meus irmãos, pelas conversas descontraídas, risadas e até brigas (por que não?), nos raros fins de semana de convívio.

Aos meus amigos, pela presença, sempre tão oportuna, naqueles momentos em que é preciso deixar de lado os estudos.

Aos amigos Emílio, Miburge e Roberto. Obrigado por fazerem parte da minha vida.

À Carol, Mônica, Regina e Solange, do Departamento de Medicina Social, pela prontidão e pela boa-vontade demonstradas durante a elaboração deste trabalho.

À Fundação de Apoio ao Ensino, Pesquisa e Assistência, pelo auxílio financeiro. 
"Chamar o especialista em Estatística depois que o experimento foi feito, pode ser o mesmo que pedir a ele para fazer um exame post mortem. Talvez ele consiga dizer do que foi que o experimento morreu."

R. A. Fisher 


\title{
Resumo
}

\author{
ARAGON, D. C. Avaliação de métodos estatísticos aplicados ao estudo de testes \\ diagnósticos na presença do viés de verificação. 2007. 114f. Dissertação (mestrado) \\ - Faculdade de Medicina de Ribeirão Preto, Universidade de São Paulo, Ribeirão Preto, \\ 2007.
}

O estudo de métodos estatísticos na avaliação de métodos diagnósticos tem aumentado consideravelmente nas últimas décadas. Desde o início, quando Yerushalmy (1947) publicou seu trabalho sobre confiabilidade do roentgenograma na identificação da tuberculose, novas metodologias surgiram para que fosse possível a obtenção de valores de sensibilidade e especificidade de testes diagnósticos. A sensibilidade é definida como a probabilidade de o teste sob investigação fornecer um resultado positivo, dado que o indivíduo é realmente portador da enfermidade. A especificidade, por sua vez, é definida como a probabilidade de o teste fornecer um resultado negativo, dado que o indivíduo está livre da enfermidade. Na prática, é comum ocorrerem situações em que uma proporção de indivíduos selecionados não pode ter o estado real da doença verificado, por se tratar de procedimentos invasivos, como no diagnóstico de câncer de pulmão, ou quaisquer outros casos em que são envolvidos riscos, portanto não praticáveis, nem éticos, ou ainda por serem de alto custo. Assim, em vez de se contornar o problema, muitos estudos de avaliação de performance de testes diagnósticos são elaborados apenas com informações de indivíduos verificados. Esse procedimento pode levar a resultados viesados. É o chamado viés de verificação, que consiste no cálculo de estimativas de sensibilidade e especificidade de testes diagnósticos quando apenas os indivíduos verificados pelo padrão ouro são inseridos na análise e os não verificados são descartados ou considerados livres de doença. Este trabalho apresenta uma revisão das metodologias já propostas para se calcularem a sensibilidade e a especificidade quando existe o viés de verificação, bem como uma análise detalhada da influência da proporção de indivíduos não verificados, o efeito do tamanho amostral e a escolha de distribuições a priori, quando utilizada a metodologia bayesiana, no cálculo dessas estimativas. Também foi introduzida uma metodologia, sob enfoque bayesiano, para a estimação das medidas de desempenho de dois testes diagnósticos, na presença do viés de verificação.

Palavras - chave: Viés de verificação, Testes diagnósticos, Métodos bayesianos. 


\title{
Abstract
}

\author{
ARAGON, D. C. Evaluation of statistical methods applied to diagnostics tests \\ in the presence of the verification bias. 2007. 114p. Dissertation (master degree) - \\ Faculty of Medicine of Ribeirão Preto, University of São Paulo, Ribeirão Preto, 2007.
}

The study of statistical methods on diagnostic tests evaluation has increased in the last decades. Since the beginning, when Yerushalmy (1947) published his work about trustworthiness of the roentgenogram in the identification of the tuberculosis, new methodologies had appeared and so that it was possible to get values of sensitivity and specificity of diagnostic tests. Sensitivity is defined as the probability of the test under inquiry supply a positive result, since that the individual is really carrying on the disease. The specificity, in the other hand, is defined as the probability of the test supply a negative result, since that the individual is free of the disease. In practice, it is usual to occur situations where a proportion of selected individuals cannot have verified the real state of the illness, to the fact that the verification test can be an invasive procedure, as in the diagnosis of lung cancer, or any other cases where risks are involved, therefore not practicable, nor ethical, or still procedures with high cost. Thus, instead of solve the problem, many studies of evaluation of performance of diagnostic tests are elaborated only using the information of verified individuals. This procedure can leads to biased results. This is known as verification bias, that consists of the calculation of estimates of sensitivity and specificity of diagnostic tests when only the individuals verified by the gold standard test are inserted in the analysis and the unverified ones, discarded or considered that they are free of the disease. This work presents a revision of the methodologies already proposed to calculate sensitivity and the specificity in the presence of the verification bias, as well as a detailed analysis of the influence of the proportion of individuals not verified, the effect of the sample size and the influence of choosing different prior densities, when using the bayesian methodology, in the calculation of these estimates. It was also introduced a bayesian methodology to estimate performance measures of two diagnostic tests when the verification bias is present.

Keywords: Verification bias, Diagnostic tests, Bayesian methods. 


\section{Lista de Figuras}

1 Valores preditivos positivos e negativos, de acordo com a prevalência. . . . 24

2 Gráficos de autocorrelações . . . . . . . . . . . . . . . . . . . . . . . 113

3 Gráficos de convergência . . . . . . . . . . . . . . . . . . . . 114 


\section{Lista de Tabelas}

1 Freqüência de indivíduos no cruzamento dos resultados do padrão ouro e teste diagnóstico. . . . . . . . . . . . . . . . . . . . . . . . . . . 17

2 Freqüências dos resultados do teste de tolerância ao exercício e diagnóstico de doença coronariana. . . . . . . . . . . . . . . . . . . 23

3 Freqüências dos resultados do $\mathrm{FEV}_{1}$ e diagnóstico correto. . . . . . . . . . 24

4 Freqüências dos resultados dos relatos médicos e informações das certidões de nascimento. . . . . . . . . . . . . . . . . . 25

5 Freqüências dos resultados do CAGE e grupos de alcoólatras e não alcoólatras. 25

6 Freqüências dos resultados laboratoriais e presença de cocaína. . . . . . . . 26

7 Freqüências dos resultados do nível sérico de digoxina e diagnóstico de intoxicação. . . . . . . . . . . . . . . . . . . 26

8 Freqüências dos resultados da ventriculografia radionuclídica e doença da artéria coronária. . . . . . . . . . . . . . . . . . . . . . 27

9 Freqüências dos resultados dos raios-X e tuberculose. . . . . . . . . . . . 27

10 Freqüências dos resultados da cintilografia e diagnóstico de doença hepática. 29

11 Freqüências dos resultados do diagnóstico histológico e do HC-II. . . . . . . 30

12 Freqüências absolutas para o cruzamento das variáveis $D, T$ e $V$. . . . . 35

13 Probabilidades para o cruzamento das variáveis $D, T$ e $V$. . . . . . . . 38

14 Estimativas de sensibilidade e especificidade - Método de Begg e Greenes. . 45

15 Limites para sensibilidade e especificidade - Método de Zhou. . . . . . . . . 46

16 Estimativas de sensibilidade e especificidade - Método de Begg e Greenes. . 47

17 Limites para sensibilidade e especificidade - Método de Zhou. . . . . . . . . 47

18 Estimativas de sensibilidade e especificidade - Método de Begg e Greenes. . 48

19 Limites para sensibilidade e especificidade - Método de Zhou. . . . . . . . . 49

20 Sumários a posteriori e intervalos de credibilidade 95\% (ICr 95\%): (a) resultados obtidos a partir de distribuições a priori não informativas; (b) resultados obtidos a partir de distribuições a priori informativas para $S_{E}$

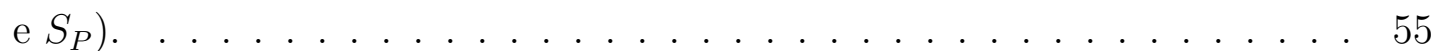

21 Sumários a posteriori e intervalos de credibilidade 95\% (ICr 95\%): (a) resultados obtidos a partir de distribuições a priori não informativas; (b) resultados obtidos a partir de distribuições a priori informativas para $S_{E}$

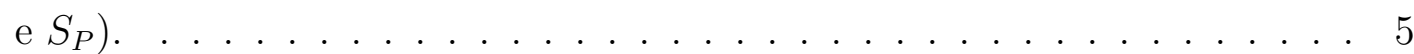

22 Resultados das simulações para cada porcentagem de verificação - dados de WEINER et al. (1979) - distribuições a priori não informativas. . . . . 58

23 Resultados das simulações para cada porcentagem de verificação - dados de WEINER et al. (1979) - disribuições a priori informativas (amostra aleatória de 10\%). 
24 Resultados das simulações para cada porcentagem de verificação - dados de WEINER et al. (1979) - distribuições a priori informativas (amostra aleatória de 20\%). . . . . . . . . . . . . . . . . 5 5

25 Resultados das simulações para cada porcentagem de verificação - dados de WEINER et al. (1979) - distribuições a priori informativas (amostra aleatória de 30\%).

26 Resultados das simulações para cada porcentagem de verificação - dados de WEINER et al. (1979) - distribuições a priori informativas (amostra aleatória de 40\%).

27 Resultados das simulações para cada porcentagem de verificação - dados de HIGGINS e KELLER (1973) - distribuições a priori não informativas.

28 Resultados das simulações para cada porcentagem de verificação - dados de HIGGINS e KELLER (1973) - distribuições a priori informativas (amostra aleatória de 10\%).

29 Resultados das simulações para cada porcentagem de verificação - dados de HIGGINS e KELLER (1973) - distribuições a priori informativas (amostra aleatória de 20\%).

30 Resultados das simulações para cada porcentagem de verificação - dados de HIGGINS e KELLER (1973) - distribuições a priori informativas (amostra aleatória de 30\%).

31 Resultados das simulações para cada porcentagem de verificação - dados de HIGGINS e KELLER (1973) - distribuições a priori informativas (amostra aleatória de 40\%).

32 Resultados das simulações para cada porcentagem de verificação - dados de BEGG e MCNEIL (1988) - distribuições a priori não informativas.

33 Resultados das simulações para cada porcentagem de verificação - dados de BEGG e MCNEIL (1988) - distribuições a priori informativas (amostra aleatória de 10\%). . . . . . . . . . . . . . . .

34 Resultados das simulações para cada porcentagem de verificação - dados de BEGG e MCNEIL (1988) - distribuições a priori informativas (amostra aleatória de 20\%).

35 Resultados das simulações para cada porcentagem de verificação - dados de BEGG e MCNEIL (1988) - distribuições a priori informativas (amostra aleatória de $30 \%)$.

36 Resultados das simulações para cada porcentagem de verificação - dados de BEGG e MCNEIL (1988) - distribuições a priori informativas (amostra aleatória de 40\%). 
37 Resultados das simulações para cada porcentagem de verificação - dados de WEINER et al. (1979) - distribuições a priori não informativas (diferentes probabilidades de verificação). . . . . . . . . . . . . . . . . . 67

38 Resultados das simulações para cada porcentagem de verificação - dados de WEINER et al. (1979) - distribuições a priori não informativas (diferentes probabilidades de verificação).

39 Resultados das simulações para cada porcentagem de verificação - dados de WEINER et al. (1979) - distribuições a priori não informativas (diferentes probabilidades de verificação).

40 Resultados das simulações para cada porcentagem de verificação - dados de WEINER et al. (1979) - distribuições a priori não informativas (diferentes probabilidades de verificação).

41 Resultados das simulações para cada porcentagem de verificação - dados de HIGGINS e KELLER (1973) - distribuições a priori não informativas (diferentes probabilidades de verificação). . . . . . . . . . . . . . .

42 Resultados das simulações para cada porcentagem de verificação - dados de HIGGINS e KELLER (1973) - distribuições a priori não informativas (diferentes probabilidades de verificação).

43 Resultados das simulações para cada porcentagem de verificação - dados de HIGGINS e KELLER (1973) - distribuições a priori não informativas (diferentes probabilidades de verificação).

44 Resultados das simulações para cada porcentagem de verificação - dados de HIGGINS e KELLER (1973) - distribuições a priori não informativas (diferentes probabilidades de verificação).

45 Resultados das simulações para cada porcentagem de verificação - dados de BEGG e MCNEIL (1988) - distribuições a priori não informativas (diferentes probabilidades de verificação). . . . . . . . . . . . . . . .

46 Resultados das simulações para cada porcentagem de verificação - dados de BEGG e MCNEIL (1988) - distribuições a priori não informativas (diferentes probabilidades de verificação).

47 Resultados das simulações para cada porcentagem de verificação - dados de BEGG e MCNEIL (1988) - distribuições a priori não informativas (diferentes probabilidades de verificação).

48 Resultados das simulações para cada porcentagem de verificação - dados de BEGG e MCNEIL (1988) - distribuições a priori não informativas (diferentes probabilidades de verificação) . . . . . . . . . . . . . . 72

49 Combinações de resultados dos testes 1 e 2, de acordo com $D$ e $V$. . . . . 78

50 Contribuições de todas as possíveis combinações entre as variáveis $V, D, T_{1}$ e $T_{2}$ à verossimilhança. . . . . . . . . . . . . . . . . . . . . 
51 Dados do estudo de HALL et al. (1996) sobre o diagnóstico da doença de Alzheimer. . . . . . . . . . . . . . . . . . 85

52 Dados do estudo de RATNAM et al. (2000) sobre o rastreamento do câncer cervical. . . . . . . . . . . . . . . . . . . 85

53 Resultados a posteriori para os dados de HALL et al. (1996) - distribuições a priori não informativas. . . . . . . . . . . . . . . . . . . 86

54 Resultados a posteriori para os dados de HALL et al. (1996). . . . . . . . 87

55 Resultados a posteriori para os dados de HALL et al. (1996). . . . . . . . 87

56 Resultados a posteriori para os dados de HALL et al. (1996). . . . . . . . 88

57 Resultados a posteriori para os dados de HALL et al. (1996). . . . . . . . . 88

58 Resultados a posteriori para os dados de HALL et al. (1996). . . . . . . . 89

59 Resultados a posteriori para os dados de HALL et al. (1996). . . . . . . . 89

60 Resultados a posteriori para os dados de RATNAM et al. (2000) - distribuições a priori não informativas. . . . . . . . . . . . . . . . . . . . . . . 90

61 Resultados a posteriori para os dados de RATNAM et al. (2000). . . . . . 90

62 Resultados a posteriori para os dados de RATNAM et al. (2000). . . . . . 91

63 Resultados a posteriori para os dados de RATNAM et al. (2000). . . . . . 91

64 Resultados a posteriori para os dados de RATNAM et al. (2000). . . . . . 92

65 Resultados a posteriori para os dados de RATNAM et al. (2000). . . . . . 92

66 Resultados a posteriori para os dados de RATNAM et al. (2000). . . . . . 93

67 Freqüência do cruzamento entre $D$ e $T$ - dados completos. . . . . . . . . . 93

68 Freqüência do cruzamento entre $D, T$ e $V$ - dados simulados. . . . . . . . . 94

69 Resultados a posteriori - dados simulados - distribuições a priori não informativas. . . . . . . . . . . . . . . . . . . . 95

70 Resultados a posteriori - dados simulados - distribuições a priori não informativas. . . . . . . . . . . . . . . . . . . 95

71 Resultados a posteriori - dados simulados - distribuições a priori não informativas. . . . . . . . . . . . . . . . . . 96

72 Resultados a posteriori - dados simulados - distribuições a priori não informativas. . . . . . . . . . . . . . . . . . . . 96

73 Resultados a posteriori - dados simulados - distribuições a priori não informativas. . . . . . . . . . . . . . . . . . . 97

74 Resultados a posteriori - dados simulados - distribuições a priori informativas. . . . . . . . . . . . . . . . . . 97

75 Resultados a posteriori - dados simulados - distribuições a priori informativas. . . . . . . . . . . . . . . . . . . . . . 98

76 Resultados a posteriori - dados simulados - distribuições a priori informativas. . . . . . . . . . . . . . . . . . . . . . . 98 
77 Resultados a posteriori - dados simulados - distribuições a priori informativas. . . . . . . . . . . . . . . . . . . . 99

78 Resultados a posteriori - dados simulados - distribuições a priori informativas. . . . . . . . . . . . . . . . . . . . 99

79 Resultados a posteriori - dados simulados - distribuições a priori informativas. . . . . . . . . . . . . . . . . . . . . 100

80 Resultados a posteriori - dados simulados - distribuições a priori informativas. . . . . . . . . . . . . . . . . . . . . . 100

81 Resultados a posteriori - dados simulados - distribuições a priori informativas. . . . . . . . . . . . . . . . . . . . . 100

82 Resultados a posteriori - dados simulados - distribuições a priori informativas. . . . . . . . . . . . . . . . . . . . . 101

83 Contribuições de todas as possíveis combinações entre as variáveis $V, D, T_{1}$ e $T_{2}$ à verossimilhança. . . . . . . . . . . . . . . . . . . . . . . . . . . . . 102

84 Freqüência do cruzamento entre $D, T$ e $V$ - dados simulados. . . . . . . . . 102

85 Resultados a posteriori - dados simulados - distribuições a priori não informativas - testes dependentes. . . . . . . . . . . . . . . . . . 103

86 Resultados a posteriori - dados simulados - distribuições a priori informativas - testes dependentes. . . . . . . . . . . . . . . . . . . . . . 104

87 Resultados a posteriori - dados simulados - distribuições a priori nãoinformativas - testes dependentes. . . . . . . . . . . . . . . . . . . 104

88 Resultados a posteriori - dados simulados - distribuições a priori informativas - testes dependentes. . . . . . . . . . . . . . . . . . . 105 


\section{Lista de Abreviaturas e Siglas}

IC........... Intervalo de confiança

VPP........ Valor preditivo positivo

VPN....... Valor preditivo negativo

RV .......... Razão de verossimilhanças

ROC........ Receiver operation curve

DPOC...... Doença pulmonar obstrutiva crônica

$F E V_{1} \ldots \ldots$. Volume forçado de fluxo expiratório em 1 segundo

CAGE..... Cut down, annoyed by criticism, guilty and eye-opener

HPV ........ Papilomavírus humano

DNA........ Ácido desoxirribonucléico

HC-II....... Segunda geração de captura híbrida

TN......... Translucência nucal

PSA........ Prostate-specific antigen

3MS......... Modified mini-mental state examination

B\&G....... Begg e Greenes

SPECT.... Single photon emission computed tomographic

MCAR.... Missing completely at random

MAR...... Missing at random

NI........... Non-ignorable mising

ICr $95 \% \ldots$ Intevalo de credibilidade $95 \%$

P.O......... Padrão ouro 


\section{Sumário}

1 Introdução 16

1.1 Sensibilidade e Especificidade . . . . . . . . . . . . . . . . . . . 17

1.2 Valores Preditivos . . . . . . . . . . . . . . . . . . . . . . . . . 19

1.3 Razão de Verossimilhanças . . . . . . . . . . . . . . . . . . . . . 22

1.4 Exemplos da Literatura Médica . . . . . . . . . . . . . . . . . . . . 23

1.5 O Viés de Verificação . . . . . . . . . . . . . . . . . . . . . . . . . . . 28

1.6 Tipos de Missing . . . . . . . . . . . . . . . . . . . . . . . . . . . 31

1.7 Métodos Estatísticos nos Estudos em que o Viés de Verificação está Presente 32

2 Avaliação de Testes Diagnósticos na Presença do Viés de Verificação 35

2.1 Notação . . . . . . . . . . . . . . . . . . . . . . . . . 35

2.2 Método de Begg e Greenes . . . . . . . . . . . . . . . . . . . . . . . . . . . . . . . . . . . . . . . .

2.3 Método de Zhou . . . . . . . . . . . . . . . . . . . . . . . . 37

2.4 Exemplos . . . . . . . . . . . . . . . . . . . . . . . . 44

2.5 Método Bayesiano . . . . . . . . . . . . . . . . . . 49

2.5.1 Especificação das Distribuições a priori . . . . . . . . . . . . . . 54

2.6 Exemplos . . . . . . . . . . . . . . . . . . . 55

2.7 Simulações . . . . . . . . . . . . . . . . . . . . . . 56

2.7.1 Diferentes Distribuições a priori . . . . . . . . . . . . . . . 57

2.7.2 Diferentes Probabilidades de Verificação dos Indivíduos . . . . . . . 66

3 Avaliação de Dois Testes Diagnósticos na Presença do Viés de Verificação $\quad 74$

3.1 Método de Zhou . . . . . . . . . . . . . . . . . . . . . . . . 75

3.2 Método Bayesiano (testes independentes) . . . . . . . . . . . . . . . 81

3.2.1 Simulações . . . . . . . . . . . . . . . . . . . . . . 85

3.3 Método Bayesiano (testes dependentes) . . . . . . . . . . . . . . . 101

3.3.1 Simulações . . . . . . . . . . . . . . . . . . . . . . 103

4 Conclusão $\quad 106$

$\begin{array}{ll}\text { Referências Bibliográficas } & 107\end{array}$

$\begin{array}{ll}\text { Apêndice } & 113\end{array}$

$\begin{array}{ll}\text { A Gráficos de autocorrelações } & 113\end{array}$

$\begin{array}{lr}\text { B Gráficos de convergência } & 114\end{array}$ 


\section{Introdução}

Diariamente, os profissionais da área médica buscam o diagnóstico de doenças utilizando diversos métodos, como exames físicos, e procedimentos mais sofisticados, como ressonância magnética (MEDRONHO, 2004). É importante saber o quão confiáveis são os resultados desses exames, pois, havendo uma interpretação correta dos mesmos, o tratamento pode ser mais adequado. Resulta daí o interesse de se conhecer a qualidade dos instrumentos com que são feitas essas medidas (FRANCO e PASSOS, 2005).

Um teste diagnóstico, na literatura médica, é um procedimento que indica se um paciente tem ou não uma característica ou doença de interesse. A sensibilidade e a especificidade são medidas que traduzem o desempenho desse procedimento.

A sensibilidade $\left(S_{E}\right)$ é definida como a probabilidade de o teste sob investigação fornecer um resultado positivo, dado que o indivíduo é realmente portador da enfermidade, ou seja, se um paciente é portador do HIV, um teste de alta sensibilidade é aquele que, também com alta probabilidade, acusa a presença do vírus nesse indivíduo; ou, se um paciente é hipertenso, ele acusaria valores de pressão arterial acima dos normais.

A especificidade $\left(S_{P}\right)$, porém, é definida como a probabilidade de o teste fornecer um resultado negativo, dado que o indivíduo está livre da enfermidade. A partir dos exemplos anteriores, um instrumento de alta especificidade é aquele que, com alta probabilidade, fornece resultado negativo para HIV e valores de pressão nos níveis normais, para indivíduos que, respectivamente, não são portadores do vírus, nem são hipertensos.

Essas medidas de desempenho de um método diagnóstico têm grande importância para a saúde coletiva, pois a partir delas, por exemplo, estratégias de rastreamento de uma doença em populações específicas podem ser otimizadas. Quando o rastreamento de uma doença utiliza um método diagnóstico ou laboratorial com sensibilidade e especificidade conhecidas, a chance de um indivíduo classificado como doente portar a doença é dada por uma simples aplicação do Teorema de Bayes, bem conhecido entre os estudiosos dos métodos quantitativos em saúde, que trata de probabilidades condicionais. Essa 
chance é chamada de valor preditivo positivo do método (ALTMAN e BLAND, 1994), que será discutido posteriormente. Dessa maneira, nota-se que, na fase de planejamento do rastreamento, o conhecimento prévio de $S_{E}, S_{P}$ e da prevalência da doença $(\xi)$ permite uma estimativa da porcentagem de indivíduos doentes, detectada dentre os indivíduos com resultado positivo ao método.

\subsection{Sensibilidade e Especificidade}

A utilização de métodos estatísticos na avaliação de métodos diagnósticos teve origem a partir do trabalho de Yerushalmy (1947), que tratava da confiabilidade do roentgenograma na identificação da tuberculose.

A sensibilidade $\left(S_{E}\right)$ e a especificidade $\left(S_{P}\right)$ são as medidas mais comuns para se avaliar o desempenho de um teste diagnóstico, em que $S_{E}$ é a probabilidade de um indivíduo doente ser identificado corretamente pelo teste, e $S_{P}$ é a probabilidade de um indivíduo saudável ser identificado pelo mesmo teste. Essas duas medidas são comparadas, usualmente, com os resultados de um teste de referência chamado de "padrão ouro", que é um procedimento capaz de classificar corretamente indivíduos doentes e não doentes. Seja $T$ o resultado positivo para o teste, $\bar{T}$ o resultado negativo, $D$ o estado positivo para a doença e $\bar{D}$ o estado negativo. A Tabela 1 apresenta as freqüências dos possíveis resultados do cruzamento entre o teste em questão e o padrão ouro.

Tabela 1: Freqüência de indivíduos no cruzamento dos resultados do padrão ouro e teste diagnóstico.

\begin{tabular}{ccccc}
\hline & \multicolumn{3}{c}{ Padrão Ouro } & Total \\
\cline { 2 - 4 } & & $D$ & $D$ & \\
\hline Teste Diagnóstico & $T$ & $a$ & $b$ & $a+b$ \\
& $\bar{T}$ & $c$ & $d$ & $c+d$ \\
\hline Total & & $a+c$ & $b+d$ & $a+b+c+d$ \\
\hline
\end{tabular}

A sensibilidade pode ser escrita como

$$
S_{E}=P(T \mid D)=\frac{P(T D)}{P(D)}
$$

em que $P(D)$ é a prevalência, denotada por $\xi$. 
Considerando a Tabela 1, uma estimativa de $S_{E}$ é obtida por

$$
\widehat{S_{E}}=\frac{a}{a+c}
$$

Já a especificidade, que é a proporção de verdadeiros negativos, identificada corretamente pelo teste, é dada por

$$
S_{P}=P(\bar{T} \mid \bar{D})=\frac{P(\overline{T D})}{P(\bar{D})}
$$

e pode ser estimada por

$$
\widehat{S_{P}}=\frac{d}{b+d}
$$

Intervalos de confiança (IC) para sensibilidade e especificidade podem ser calculados. Para valores entre 0,3 e 0,7, Fleiss et al. (2003) mostraram que uma aproximação assintótica para o $\mathrm{IC}_{(1-\alpha) 100 \%}$ é dada por

$$
\widehat{p}-z_{\alpha / 2} \sqrt{\frac{\widehat{p}(1-\widehat{p})}{n_{A}}}-\frac{1}{2 n_{A}} ; \widehat{p}+z_{\alpha / 2} \sqrt{\frac{\widehat{p}(1-\widehat{p})}{n_{A}}}+\frac{1}{2 n_{A}},
$$

em que $\widehat{p}$ é a estimativa da proporção de interesse (sensibilidade ou especificidade), $n_{A}=a+c$, no caso da sensibilidade, e $n_{A}=b+d$, no caso da especificidade; $z$ é o valor que limita uma área de $\alpha / 2$ na extremidade superior da distribuição normal padrão. O termo $1 / 2 n_{A}$ é uma correção de continuidade utilizada, para que haja uma melhor aproximação assintótica à distribuição normal padrão. Nota-se que a correção de continuidade é útil quando utilizadas amostras pequenas. Quando $n_{A}$ é grande, a correção de continuidade aproxima-se de zero. Nos problemas de avaliação de desempenho de testes diagnósticos esses intervalos não são indicados, dado que, freqüentemente, há testes com sensibilidade e/ou especificidade superior a 70\% (ver FLEISS, et al., 2003, pág. 28). Além do mais, quando são encontrados valores muito altos para essas medidas, o limite superior pode ultrapassar o 100\%, sendo necessário truncar o intervalo nesse valor. Quando isso acontece, a probabilidade de cobertura passa a não ser mais a nominal. Alternativamente, os limites 
inferiores e superiores (LI e LS) dos IC para sensibilidade e especificidade, obtidos pelo método da quantidade pivotal, são dados, respectivamente, por

$$
L I_{p}=\frac{\left(2 n_{A} \widehat{p}+z_{\alpha / 2}^{2}-1\right)-z_{\alpha / 2} \sqrt{z_{\alpha / 2}^{2}-\left(2+\frac{1}{n_{A}}\right)+4 \widehat{p}\left[n_{A}(1-\widehat{p})+1\right]}}{2\left(n_{A}+z_{\alpha / 2}^{2}\right)}
$$

e

$$
L S_{p}=\frac{\left(2 n_{A} \widehat{p}+z_{\alpha / 2}^{2}+1\right)+z_{\alpha / 2} \sqrt{z_{\alpha / 2}^{2}+\left(2+\frac{1}{n_{A}}\right)+4 \widehat{p}\left[n_{A}(1-\widehat{p})-1\right]}}{2\left(n_{A}+z_{\alpha / 2}^{2}\right)}
$$

Em casos em que o interesse é a obtenção do diagnóstico precoce de uma doença (rastreamentos), é necessário um teste com alta sensibilidade. Como exemplo, podem ser citados os bancos de sangue, no intuito de se diagnosticar hepatite $\mathrm{C}$ em doadores (FRANCO e PASSOS, 2005).

Já em situações em que o diagnóstico implica em um procedimento mais invasivo, o interesse maior pode ser direcionado para a especificidade. Assim, um teste sorológico para HIV deve ter alta especificidade, dadas as conseqüências do diagnóstico para o paciente, tanto no aspecto médico quanto no social (FRANCO e PASSOS, 2005).

\subsection{Valores Preditivos}

Na prática clínica e em planejamentos de políticas de saúde, dado o resultado do teste, convém conhecer a probabilidade de um indivíduo ter ou não uma doença. A sensibilidade e a especificidade não são capazes de fornecer essa informação. Para isso, são utilizados os valores preditivos. Um resultado falso positivo ou um falso negativo está, freqüentemente, associado a maiores gastos com saúde coletiva (KOSINSKI e BARNHART, 2003).

O valor preditivo positivo $(V P P)$ é dado pela proporção de indivíduos com resultado positivo para o teste e que realmente estão doentes. A partir do Teorema de Bayes,

$$
V P P=P(D \mid T)=\frac{P(D, T)}{P(T)}=\frac{P(T \mid D) P(D)}{P(T \mid D) P(D)+[1-P(\bar{T} \mid \bar{D})][1-P(D)]},
$$

ou seja, 


$$
V P P=\frac{S_{E} \xi}{S_{E} \xi+\left(1-S_{P}\right)(1-\xi)}
$$

O intervalo de confiança $(100-\alpha) \%$, assintótico, para o VPP, obtido a partir do método delta, é dado por

$$
\widehat{V P P}-z_{\alpha / 2} \sqrt{\operatorname{Var} \widehat{(V P P})} ; \widehat{V P P}+z_{\alpha / 2} \sqrt{\operatorname{Var} \widehat{(V P P})}
$$

em que

$$
\operatorname{Var} \widehat{(V P P})=\frac{\left[\widehat{S_{E}}\left(1-\widehat{S_{P}}\right)\right]^{2}[\widehat{\xi}(1-\widehat{\xi}) / n]}{\left[\widehat{S_{E}} \widehat{\xi}+\left(1-\widehat{S_{P}}\right)(1-\widehat{\xi})\right]^{4}}+\frac{\left[\hat{\xi}(1-\widehat{\xi})\left(1-\widehat{S_{P}}\right)\right]^{2}\left[\widehat{S_{E}}\left(1-\widehat{S_{E}}\right) / n\right]}{\left[\widehat{S_{E}} \widehat{\xi}+\left(1-\widehat{S_{P}}\right)(1-\widehat{\xi})\right]^{4}}+\frac{\left[\widehat{S_{E}} \widehat{\xi}(1-\widehat{\xi})\right]^{2}\left[\widehat{S_{P}}\left(1-\widehat{S_{P}}\right) / n\right]}{\left[\widehat{S_{E}} \widehat{\xi}+\left(1-\widehat{S_{P}}\right)(1-\widehat{\xi})\right]^{4}}
$$

O valor preditivo negativo $(V P N)$ é a proporção de indivíduos com resultado negativo para o teste e que realmente não estão doentes. É dado por

$$
V P N=P(\bar{D} \mid \bar{T})=\frac{P(\bar{D}, \bar{T})}{P(\bar{T})}=\frac{P(\bar{T} \mid \bar{D})[1-P(D)]}{P(\bar{T} \mid \bar{D})[1-P(D)]+[1-P(T \mid D)] P(D)},
$$

ou seja

$$
V P N=\frac{S_{P}(1-\xi)}{S_{P}(1-\xi)+\left(1-S_{E}\right) \xi}
$$

O intervalo de confiança $(100-\alpha) \%$, assintótico, para o $V P N$, obtido a partir do método delta, é dado por

$$
\widehat{V P N}-z_{\alpha / 2} \sqrt{\operatorname{Var(VPN)}} ; \widehat{V P N}+z_{\alpha / 2} \sqrt{\operatorname{Var(VPN)}}
$$

em que

$$
\operatorname{Var} \widehat{(V P} P)=\frac{\left.\left[\widehat{S_{P}}\left(1-\widehat{S_{E}}\right)\right]^{2} \widehat{\xi}(1-\widehat{\xi}) / n\right]}{\left[\widehat{S_{P}}(1-\widehat{\xi})+\left(1-\widehat{S_{E}}\right) \hat{\xi}\right]^{4}}+\frac{\left\{\widehat{\xi}\left[\widehat{S_{P}}(1-\widehat{\xi})\right]\right\}^{2}\left[\widehat{S_{E}}\left(1-\widehat{S_{E}}\right) / n\right]}{\left[\widehat{S_{P}}(1-\widehat{\xi})+\left(1-\widehat{S_{E}}\right) \widehat{\xi}\right]^{4}}+\frac{\left[(1-\widehat{\xi})\left(1-\widehat{S_{E}}\right) \widehat{\xi}\right]^{2}\left[\widehat{S_{P}}\left(1-\widehat{S_{P}}\right) / n\right]}{\left[\widehat{S_{P}}(1-\widehat{\xi})+\left(1-\widehat{S_{E}}\right) \widehat{\xi}\right]^{4}}
$$

As estimativas de $\operatorname{Var}(V P P)$ e de $\operatorname{Var}(V P N)$ foram obtidas a partir da expansão de 
séries de Taylor. Seja a função $f$ infinitamente diferenciável em um intervalo aberto $J$ e seja $h$ um número em $J$. Então a série de Taylor para $f$ em $h$ é dada por:

$$
f(x)=f(h)+f^{\prime}(a)(x-h)+\frac{f^{\prime \prime}(h)(x-h)^{2}}{2 !}+\ldots+\frac{f^{(n)}(h)(x-h)^{n}}{n !} .
$$

Seja $X$ uma variável aleatória com $E(X)=\mu_{x}$ e $\operatorname{Var}(X)=\sigma_{x}^{2}$. Seja, também, $Y=$ $H(x)$, em que $H$ é uma função derivável, pelo menos, no ponto $x=\mu_{x}$. Desenvolvendo $H$ em série de Taylor, em $x=\mu_{x}$, tem-se

$$
Y=H\left(\mu_{x}\right)+(X-\mu) H^{\prime}\left(\mu_{x}\right)+\varepsilon
$$

em que $\varepsilon$ é um resto. Se $\varepsilon$ for desconsiderado, então tem-se

$$
\begin{aligned}
\operatorname{Var}(Y) & \simeq \operatorname{Var}\left[H\left(\mu_{x}\right)+(X-\mu) H^{\prime}\left(\mu_{x}\right)\right] \\
& =\left[H^{\prime}\left(\mu_{x}\right)\right]^{2} \operatorname{Var}(X-\mu)=\left[H^{\prime}\left(\mu_{x}\right)\right]^{2} \sigma_{x}^{2} .
\end{aligned}
$$

Assim, por exemplo, $\sigma_{V P P}^{2}$ seria dado por

$$
\sigma_{V P P}^{2}=\left(\frac{\partial V P P}{\partial \xi}\right)^{2} \sigma_{\xi}^{2}+\left(\frac{\partial V P P}{\partial S_{E}}\right)^{2} \sigma_{S_{E}}^{2}+\left(\frac{\partial V P P}{\partial S_{P}}\right)^{2} \sigma_{S_{P}}^{2}
$$

Os intervalos de confiança para os valores preditivos não podem ser obtidos a partir de (4) porque o $V P P$ e o $V P N$ são calculados utilizando-se a $S_{E}$, a $S_{P}$ e ainda a $\xi$, então deve-se levar em conta a variabilidade amostral destas três medidas para se obter os intervalos de confiança.

Assim, pode-se verificar, a partir de (5), que, se a prevalência ( $\xi$ ) é muito baixa, o $V P P$ não vai ser próximo de 1 , mesmo que $S_{E}$ e $S_{P}$ sejam altas. Portanto, nos rastreamentos em uma população, é inevitável haver muitos indivíduos com resultado positivo para o teste, mas que são falsos positivos. A partir das equações para os cálculos dos valores preditivos, pode-se afirmar que, se a $S_{E}$ for baixa, sua contribuição para o $V P P$ também será baixa; se a prevalência for baixa, maior será a contribuição da $S_{P}$, no cálculo do $V P P$. 
Conclui-se, então, que, se a utilização prática do teste exige um alto $V P P$, é importante que, para um bom desempenho diagnóstico, a decisão de quão altas devem ser a $S_{E}$ e a $S_{P}$ do teste seja fundamentada em uma estimativa prévia da prevalência da doença.

A prevalência pode ser interpretada como a probabilidade de um indivíduo ter uma doença, mas antes de o teste ser aplicado e é chamada de probabilidade de doença a priori. Os $V P P$ e $V P N$ são conhecidos como probabilidade de doença a posteriori.

\subsection{Razão de Verossimilhanças}

Para qualquer teste, é possível comparar a probabilidade de se obter um resultado positivo, dado que um indivíduo realmente tem uma doença, com a probabilidade do teste dar positivo, dado que ele é saudável. A razão dessas probabilidades é chamada razão de verossimilhanças $(R V)$ (ver FLEISS et al., 2003) e é dada por

$$
R V=\frac{P(T \mid D)}{[1-P(\bar{T} \mid \bar{D})]}
$$

ou seja

$$
R V=\frac{S_{E}}{1-S_{P}}
$$

Assim, um valor alto de $R V$ indica que o teste é útil, mas não necessariamente que um resultado positivo seja um bom indicador da presença da doença. Quando $R V=1$, o teste em questão se mostra sem utilidade alguma, já que tende a produzir os mesmos resultados, tanto para doentes como para não-doentes. Por meio do método delta, é possível obter-se o intervalo de confiança para a $R V$ (ver SIMEL et al., 1991), dado por

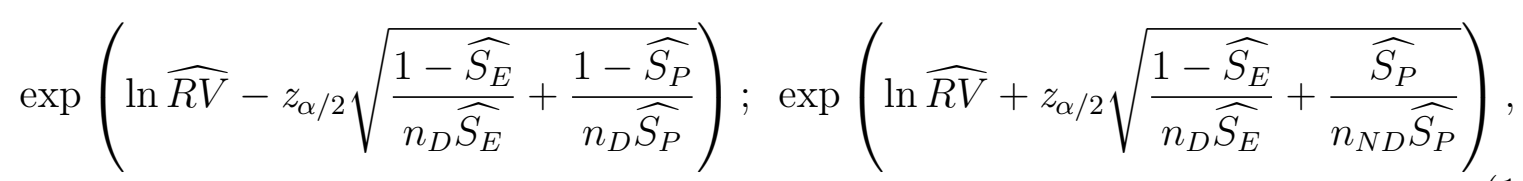

em que $n_{D}$ e $n_{N D}$ são, respectivamente, o número total de doentes e de não-doentes, e $z_{\alpha / 2}$ é o percentil da distribuição normal padrão para um coeficiente $(1-\alpha) 100 \%$ de confiança. 
Até agora, considerou-se que os testes diagnósticos são baseados em respostas binárias, do tipo "positivo" ou "negativo". Muitos deles, entretanto, são quantitativos, dados em escala contínua. A mesma abordagem estatística citada anteriormente pode ser utilizada se um ponto de corte puder ser estabelecido entre os indivíduos normais e os doentes, o que não é algo trivial. A curva ROC (receiver operating characteristic) é uma maneira de se obter esse ponto, por meio de um gráfico da sensibilidade versus 1 - especificidade, obtidas para cada observação. Como esse não é o objetivo maior deste trabalho, essa metodologia apenas será citada aqui.

\subsection{Exemplos da Literatura Médica}

A seguir, são dados alguns exemplos de aplicações reais utilizando-se os conceitos de sensibilidade e especifidade.

Exemplo 1) Weiner et al. (1979) publicaram um estudo em que foram comparados, por meio de um teste de tolerância ao exercício, indivíduos com e sem doença coronariana.

Tabela 2: Freqüências dos resultados do teste de tolerância ao exercício e diagnóstico de doença coronariana.

\begin{tabular}{cccc}
\hline & \multicolumn{3}{c}{ Doença Coronariana } \\
\cline { 2 - 4 } Teste de Tolerância ao Exercício & Presente $(+)$ & Ausente $(-)$ & Total \\
\hline Presente (+) & 815 & 115 & 930 \\
Ausente (-) & 208 & 327 & 535 \\
\hline Total & 1023 & 442 & 1465 \\
\hline
\end{tabular}

Os valores para a sensibilidade, especificidade e seus intervalos de confiança, obtidos a partir da equação dada em (4), são:

$$
\begin{aligned}
\widehat{S_{E}} & =\frac{815}{1023}=0,797 ; I C_{S_{E} 95 \%}(0,770 ; 0,820) \\
\widehat{S_{P}} & =\frac{327}{442}=0,740 ; I C_{S_{P} 95 \%}(0,696 ; 0,777)
\end{aligned}
$$

A Figura 1 mostra a influência do valor da prevalência nos valores preditivos, já discutida anteriormente. Nota-se que quando a prevalência é menor que 10\%, o VPN é a alto, mas o VPP é baixo. 


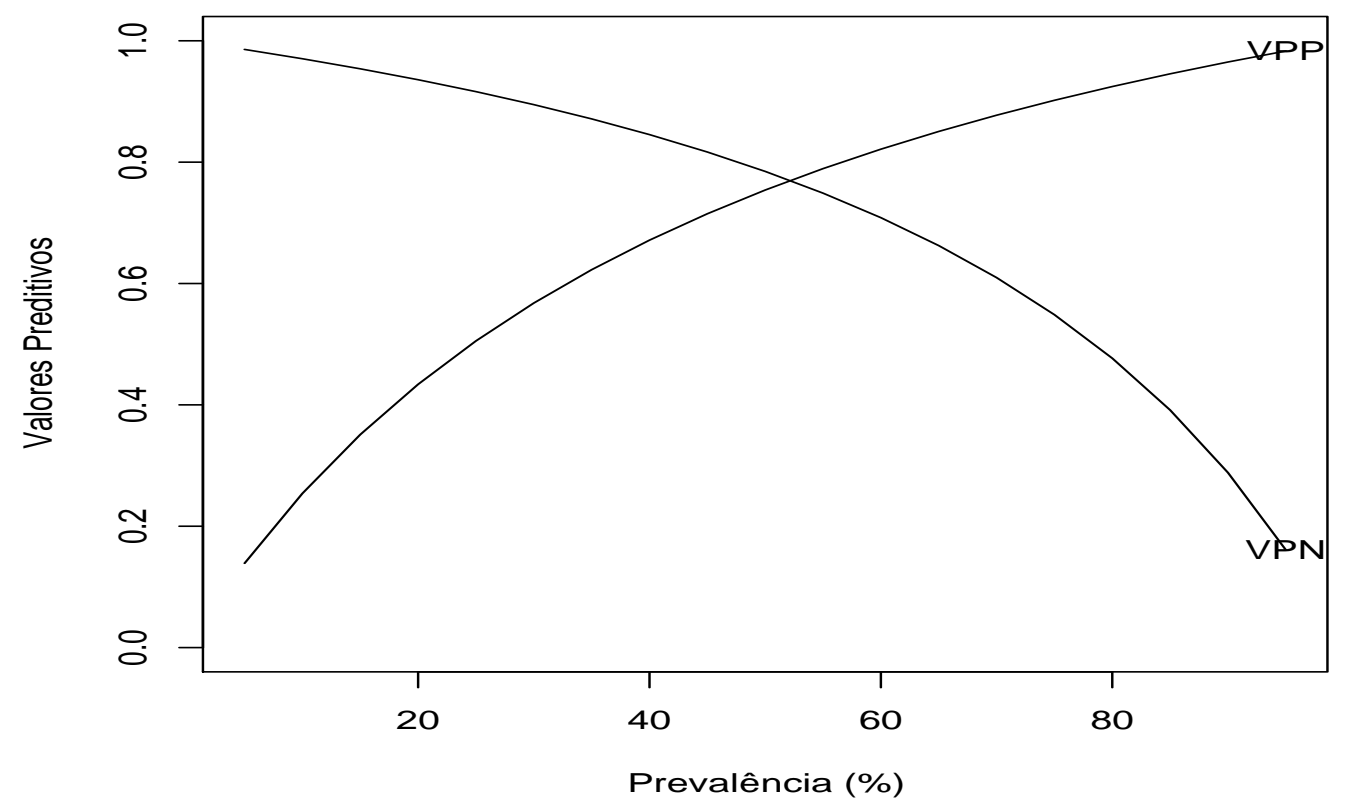

Figura 1: Valores preditivos positivos e negativos, de acordo com a prevalência.

Exemplo 2) Higgins e Keller (1973) apresentaram um trabalho em que residentes de uma cidade participaram de um rastreamento para doença pulmonar obstrutiva crônica (DPOC), diagnosticada a partir do volume forçado de fluxo expiratório em um segundo $\left(\mathrm{FEV}_{1}\right)$.

Tabela 3: Freqüências dos resultados do $\mathrm{FEV}_{1}$ e diagnóstico correto.

\begin{tabular}{cccc}
\hline & \multicolumn{3}{c}{ DPOC } \\
\cline { 2 - 4 } FEV $_{1}$ & Presente $(+)$ & Ausente (-) & Total \\
\hline$<80(+)$ & 65 & 134 & 199 \\
$\geq 80(-)$ & 256 & 1409 & 1665 \\
\hline Total & 321 & 1543 & 1864 \\
\hline
\end{tabular}

Os valores para a sensibilidade, especificidade e seus intervalos de confiança são:

$$
\begin{aligned}
& \widehat{S_{E}}=\frac{65}{321}=0,202 ; I C_{S_{E} 95 \%}(0,161 ; 0,248) \\
& \widehat{S_{P}}=\frac{1409}{1543}=0,913 ; I C_{S_{P} 95 \%}(0,898 ; 0,926)
\end{aligned}
$$

Nesse exemplo, nota-se que o teste para a doença não traz uma resposta binária, mas, sim, um valor dentro de uma escala contínua. É usual, nessas situações, buscar-se um ponto de corte ótimo para classificação dos indivíduos como positivos ou negativos. 
Também é interessante observar que, os autores do estudo, se tomassem um ponto de corte diferente de $80 \%$, poderiam obter maior sensibilidade, mas menor especificidade do teste.

Exemplo 3) Selvin (1996) mostra, em um estudo, que relatos médicos, cuidadosamente investigados a respeito de uma específica má-formação em recém nascidos, foram comparados com as informações contidas nas certidões de nascimento dos mesmos. Admite-se que os relatos médicos são mais completos e corretos.

Tabela 4: Freqüências dos resultados dos relatos médicos e informações das certidões de nascimento.

\begin{tabular}{cccc}
\hline & \multicolumn{3}{c}{ Relatos Médicos } \\
\cline { 2 - 4 } Certidão de Nascimento & Presente $(+)$ & Ausente (-) & Total \\
\hline Presente (+) & 8 & 13 & 21 \\
Ausente (-) & 19 & 374 & 393 \\
\hline Total & 27 & 387 & 414 \\
\hline
\end{tabular}

Os valores para a sensibilidade, especificidade e seus intervalos de confiança são:

$$
\begin{aligned}
\widehat{S_{E}} & =\frac{8}{27}=0,296 ; I C_{S_{E} 95 \%}(0,145 ; 0,466) \\
\widehat{S_{P}} & =\frac{374}{387}=0,966 ; I C_{S_{P} 95 \%}(0,942 ; 0,979)
\end{aligned}
$$

Exemplo 4) Um questionário (CAGE) elaborado para captar a percepção do próprio entrevistado sobre ingestão excessiva de álcool foi aplicado a pessoas, na cidade de São Paulo (MASUR e MONTEIRO, 1983). A partir de referências na literatura, ele é capaz de identificar alcoólatras e não-alcoólatras na maior parte dos casos. Havendo um grupo de alcoólatras e outro de não-alcoólatras, devidamente confirmados, obtiveram-se as seguintes freqüências:

Tabela 5: Freqüências dos resultados do CAGE e grupos de alcoólatras e não alcoólatras.

\section{Grupos}

\begin{tabular}{cccc} 
CAGE & Alcoólatras (+) & Não Alcoólatras (-) & Total \\
\hline Positivo (+) & 60 & 8 & 68 \\
Negativo (-) & 8 & 38 & 46 \\
\hline Total & 68 & 46 & 144 \\
\hline
\end{tabular}


Os valores para a sensibilidade, especificidade e seus intervalos de confiança são:

$$
\begin{aligned}
& \widehat{S_{E}}=\frac{60}{68}=0,882 ; I C_{S_{E} 95 \%}(0,776 ; 0,934) \\
& \widehat{S_{P}}=\frac{38}{46}=0,826 ; I C_{S_{P} 95 \%}(0,680 ; 0,901)
\end{aligned}
$$

Exemplo 5) Hansen et al. (1985) apresentaram um estudo cego em que treze laboratórios foram avaliados quanto à acurácia em detectar certas drogas por meio do exame de urina em pessoas. Dentre $30 \%$ e $40 \%$ das amostras avaliadas, a quantidade de droga era conhecida. No caso da cocaína, foram encontradas as seguintes freqüências:

Tabela 6: Freqüências dos resultados laboratoriais e presença de cocaína.

\begin{tabular}{cccc}
\hline & \multicolumn{3}{c}{ Cocaína } \\
\cline { 2 - 4 } Laboratórios & Presente $(+)$ & Ausente $(-)$ & Total \\
\hline Positivo (+) & 150 & 8 & 158 \\
Negativo (-) & 266 & 785 & 1051 \\
\hline Total & 416 & 793 & 1209 \\
\hline
\end{tabular}

Os valores para a sensibilidade, especificidade e seus intervalos de confiança são:

$$
\begin{aligned}
& \widehat{S_{E}}=\frac{150}{416}=0,361 ; I C_{S_{E} 95 \%}(0,315 ; 0,407) \\
& \widehat{S_{P}}=\frac{785}{793}=0,990 ; I C_{S_{P} 95 \%}(0,979 ; 0,994)
\end{aligned}
$$

Exemplo 6) Beller et al. (1971) apresentaram um trabalho em que, por meio do nível sérico de digoxina no sangue de pacientes com problemas cardíacos, puderam definir a presença ou não de intoxicação. Foi estabelecido um ponto de corte de $1,7 \mathrm{ng} / \mathrm{ml}$ para separar os normais dos intoxicados. As freqüências encontradas foram as seguintes.

Tabela 7: Freqüências dos resultados do nível sérico de digoxina e diagnóstico de intoxicação.

\begin{tabular}{cccc}
\hline & \multicolumn{3}{c}{ Intoxicação } \\
\cline { 2 - 4 } Nível de Digoxina & Presente $(+)$ & Ausente (-) & Total \\
\hline Alterado (+) & 25 & 14 & 39 \\
Normal (-) & 18 & 78 & 96 \\
\hline Total & 43 & 92 & 135 \\
\hline
\end{tabular}


Os valores para a sensibilidade, especificidade e seus intervalos de confiança são:

$$
\begin{aligned}
\widehat{S_{E}} & =\frac{25}{43}=0,581 ; I C_{S_{E} 95 \%}(0,422 ; 0,706) \\
\widehat{S_{P}} & =\frac{78}{92}=0,848 ; I C_{S_{P} 95 \%}(0,754 ; 0,903)
\end{aligned}
$$

Exemplo 7) Os dados seguintes foram tomados de um estudo (BEGG e MCNEIL, 1988) que investigou o uso de uma técnica chamada ventriculografia radionuclídica como teste de diagnóstico para se detectar doença da artéria coronária.

Tabela 8: Freqüências dos resultados da ventriculografia radionuclídica e doença da artéria coronária.

\begin{tabular}{cccc}
\hline & \multicolumn{3}{c}{ Doença Coronariana } \\
\cline { 2 - 4 } Teste & Presente $(+)$ & Ausente $(-)$ & Total \\
\hline Positivo (+) & 302 & 80 & 382 \\
Negativo (-) & 179 & 372 & 551 \\
\hline Total & 481 & 452 & 933 \\
\hline
\end{tabular}

Os valores para a sensibilidade, especificidade e seus intervalos de confiança são:

$$
\begin{aligned}
& \widehat{S_{E}}=\frac{302}{481}=0,628 ; I C_{S_{E} 95 \%}(0,583 ; 0,669) \\
& \widehat{S_{P}}=\frac{372}{452}=0,823 ; I C_{S_{P} 95 \%}(0,784 ; 0,854)
\end{aligned}
$$

Exemplo 8) Em um estudo apresentado por Yerushalmy (1947), foram obtidos raios$\mathrm{X}$ do tórax de pacientes, sendo que era sabido quais tinham tuberculose. As seguintes frequiências foram encontradas:

Tabela 9: Freqüências dos resultados dos raios-X e tuberculose.

\begin{tabular}{cccc}
\hline & \multicolumn{3}{c}{ Tuberculose } \\
\cline { 2 - 4 } Raio-X & Presente $(+)$ & Ausente (-) & Total \\
\hline Positivo (+) & 22 & 51 & 73 \\
Negativo (-) & 8 & 1739 & 1747 \\
\hline Total & 30 & 1790 & 1820 \\
\hline
\end{tabular}


Os valores para a sensibilidade, especificidade e seus intervalos de confiança são:

$$
\begin{aligned}
\widehat{S_{E}} & =\frac{22}{30}=0,733 ; I C_{S_{E} 95 \%}(0,538 ; 0,846) \\
\widehat{S_{P}} & =\frac{1739}{1790}=0,972 ; I C_{S_{P} 95 \%}(0,962 ; 0,978)
\end{aligned}
$$

\subsection{O Viés de Verificação}

Na prática, é comum ocorrerem situações em que uma proporção de indivíduos selecionados não pode ter o estado real da doença verificado, por se tratar de procedimentos invasivos (biópsia), como no diagnóstico de câncer de próstata (GUPTA e ROEHRBORN, 2004), ou quaisquer outros casos que envolvam riscos, portanto não praticáveis, nem éticos, ou, ainda, procedimentos com alto custo (SOX, 1986). Assim, em vez de se contornar o problema, muitos estudos de avaliação de desempenho de testes diagnósticos são elaborados apenas com informações de indivíduos verificados. Esse procedimento pode levar a resultados viesados. São casos em que ocorre o viés de verificação (também conhecido por work-up bias) (ver BEGG, 1987; BEGG e MC NEIL, 1988; KOSINSKI e BARNHART, 2003; FEINSTEIN, 2002).

Gupta e Roehrborn (2004) apontaram dois tipos de viés de verificação. Um primeiro caso a ser citado é o em que pacientes com resultado negativo para o teste, tidos como livres da doença, não são designados para o teste de referência. Assim, são considerados como verdadeiros negativos e incorporados na análise, levando a um aumento dos valores da sensibilidade, especificidade e valor preditivo negativo. Outro caso é aquele em que os pacientes com resultado negativo para o teste, que também não são verificados pelo teste de referência (falsos negativos e verdadeiros negativos), são excluídos da análise. Isso pode levar a um aumento no valor da sensibilidade e a um decréscimo da especificidade.

Por exemplo, Drum e Christacapoulos (1972) publicaram um estudo no qual avaliaram a eficiência da cintilografia no diagnóstico de uma patologia hepática. Participaram 650 pacientes, dentre os quais 429 tinham resultado positivo para o teste, mas apenas 263 foram relacionados para verificação; 221 tiveram indicação negativa e 81 foram relacionados 
para verificação. A Tabela 10 ilustra a situação.

Tabela 10: Freqüências dos resultados da cintilografia e diagnóstico de doença hepática.

\begin{tabular}{ccccc}
\hline & \multicolumn{2}{c}{ Verificados } & & \\
\cline { 2 - 3 } Nintilografia & \multicolumn{2}{c}{ Doença Hepática } & Verificados & \\
\cline { 2 - 3 } & Presente (+) & Ausente (-) & & Total \\
\hline Positivo (+) & 231 & 32 & 166 & 429 \\
Negativa (-) & 27 & 54 & 140 & 221 \\
\hline Total & 258 & 86 & 306 & 650 \\
\hline
\end{tabular}

Se os indivíduos não verificados fossem simplesmente desprezados, a sensibilidade estimada seria dada por $231 / 258=89,5 \%$, e a especificidade seria $54 / 86=62,8 \%$. Já, se os indivíduos com resultado negativo para a cintilografia e não verificados fossem considerados como verdadeiros negativos, os valores de sensibilidade e especificidade seriam estimados, respectivamente, por $231 / 258=89,5 \%$ e $194 / 226=85,8 \%$.Observa-se, portanto, diferentes estimativas de $S_{E}$ e $S_{P}$ nestas situações, mas ambas são tendenciosas.

Na literatura médica, é estabelecido que o papilomavírus humano (HPV) está relacionado com o câncer cervical. A relação entre o HPV e o estado biológico da doença está associada com o tipo do DNA viral. O HC-II (segunda geração de Captura Híbrida) é um método recente para detecção do DNA e da carga viral, considerado um teste diagnóstico com valores satisfatórios para sensibilidade e especificidade e também de fácil aplicabilidade. Estudos de desempenho do HC-II, na detecção de neoplasia cervical, consideram, geralmente, o diagnóstico histológico da biópsia como um padrão ouro. Entretanto, apenas mulheres com diagnóstico anormal de colposcopia ou de citologia, com suspeita de lesão de alto grau, são encaminhadas para a biópsia. Portanto, esses estudos estão sujeitos ao viés de verificação. A única maneira de se evitar esse viés é fazer com que todas as mulheres passem pelo padrão ouro, o que pode ser considerado antiético; assim, é necessário estimarem-se a $S_{E}$ e a $S_{P}$ com base em freqüências de indivíduos verificados e não verificados.

Em um segundo exemplo, dado na Tabela 11, encontram-se os resultados de um estudo realizado com 105 mulheres não gestantes, com exame sugestivo de neoplasia intraepitelial/HPV (SANTOS et al., 2003) . Todas as mulheres passaram pelo HPV-DNA HC-II e foram classificadas como negativas ou positivas para alto risco de HPV. 
Tabela 11: Freqüências dos resultados do diagnóstico histológico e do HC-II.

\begin{tabular}{ccccc}
\hline & \multicolumn{2}{c}{ Verificados } & & \\
\cline { 2 - 3 } HPV-DNA HC-II & \multicolumn{2}{c}{ Diagnóstico Histológico } & Não Verificados & \\
\cline { 2 - 3 } & Presente $(+)$ & Ausente (-) & & Total \\
\hline Positivo (+) & 17 & 36 & 3 & 56 \\
Negativo (-) & 3 & 35 & 11 & 49 \\
\hline Total & 20 & 71 & 14 & 105 \\
\hline
\end{tabular}

Se os indivíduos não verificados fossem simplesmente desprezados, a sensibilidade estimada seria dada por $17 / 20=85,0 \%$, e a especificidade seria $35 / 71=49,3 \%$. Já, se os indivíduos com resultado negativo para o HPV-DNA HC-II e não verificados fossem considerados como verdadeiros negativos, os valores de sensibilidade e especificidade seriam estimados, respectivamente, por $17 / 20=85,0 \%$ e $46 / 82=56,1 \%$.

A espessura da translucência nucal (TN), desde o começo dos anos 90, começou a ser utilizada como uma marcador para a detecção da Síndrome de Down, mas o seu uso ainda é motivo de discussão entre pesquisadores da área devido ao fato de existirem muitos estudos elaborados para se avaliar o desempenho da TN. Mol et al. (1999) observaram, a partir do levantamento de vários trabalhos, que a sensibilidade da medida da TN, para a detecção da síndrome de Down, em bebês com três meses de gestação, é superestimada quando a decisão de realizar a cariotipagem fetal (padrão ouro) depende da TN.

Aproximadamente $75 \%$ dos homens norte-americanos submetem-se ao exame de próstata através do PSA (prostate-specific antigen). Entretanto, seu uso gera algumas controvérsias, pois muitos especialistas recomendam abaixar o ponto de corte tradicional do método para 4,1 ng/ml, para que seja melhorada a sensibilidade. Assim, quando um indivíduo é tido como positivo para o teste, será submetido à biópsia. O histórico familiar para a doença, a idade e o exame de toque retal também são fatores que influenciam diretamente nessa decisão. Punglia et al. (2003) publicaram um estudo em que foi realizado um rastreamento para detecção de câncer de próstata em 6691 homens norte-americanos, utilizando o PSA. Foram selecionados 705 indivíduos para serem submetidos à biópsia, e, destes, 182 realmente eram portadores da doença.

Donald e van Til (2001) publicaram um artigo em que foram calculadas a sensibilidade e a especificidade do teste 3MS (Modified Mini-Mental State Examination), para detecção 
de demência e perda cognitiva em nove mil idosos com mais de 65 anos. Os dados foram fornecidos pelo Canadian Study of Health and Aging, e os indivíduos que tinham valores menores do que 78 para o teste foram submetidos a uma avaliação clínica e neurofisiológica (padrão ouro).

A ecocardiografia, durante o exercício físico, é largamente utilizada para se verificar se um indivíduo sofre de doença coronariana. Entretanto, esse procedimento foi validado em populações específicas. Sendo assim, a decisão de submeter os indivíduos à angiografia (padrão ouro) depende, dentre outros fatores, do resultado do teste físico (ROGER et al., 1997).

Um exemplo utilizado por Zheng et al. (2005) tratou do caso de mulheres submetidas à biópsia de mama somente se o resultado da mamografia fosse positivo ou se detectada alguma saliência por meio do auto-exame, procedimento que pode resultar no viés de verificação.

Cecil et al. (1996) obtiveram a acurácia do teste SPECT Thallium-201, para diagnóstico de doença coronariana, em um estudo envolvendo 4354 indivíduos. Nem todos eles foram submetidos ao padrão ouro (angiografia), o que pode levar ao viés de verificação na estimação das medidas de desempenho do teste.

Assim, pode-se afirmar que os estudos nos quais o viés de verificação está presente são problemas em que existem informações perdidas (missing), ou seja, não se conhece o resultado real da doença dos pacientes que não foram submetidos ao padrão ouro. A seguir, serão introduzidos alguns conceitos importantes sobre a falta de dados em análises estatísticas.

\subsection{Tipos de Missing}

Todos os métodos estatísticos voltados à estimação de medidas de desempenho diagnóstico direcionam suas estratégias ao motivo que levou o pesquisador a não verificar parte da amostra. A falta de informação sobre o verdadeiro estado da doença, para os não verificados, é tratada como missing, e o mecanismo que gerou estes é a idéia central de cada método. 
A expressão MAR (missing at random, ou missing ao acaso) indica que o fato de um indivíduo ter sido submetido ou não ao padrão ouro depende do resultado do teste sob investigação e de outras características conhecidas do indivíduo (idade, sintomas, etc.), mas não do seu verdadeiro estado (desconhecido, dado que não foi verificado).

A expressão MCAR (missing completely at random, ou missing completamente ao acaso) indica que o fato do indivíduo não ter sido verificado pelo padrão ouro não depende do resultado do teste (conhecido), nem das características do indivíduo (conhecidas) e nem de seu verdadeiro estado (desconhecido). Um outro tipo de dado perdido seria o nãoignorável (NI), que difere do MCAR pelo fato do indivíduo ser verificado não depender do resultado do teste, nem das características individuais (não conhecidas) e nem do verdadeiro estado (desconhecido).

Em complemento, quando a situação não envolve missing ao acaso, utiliza-se a expressão MNAR (missing not at random). A adequação de cada método estatístico que busca evitar o efeito do viés de verificação depende da compatibilidade de seus pressupostos com a forma em que os dados foram coletados. Não se pode esperar, por exemplo, que um método estatístico que pressupõe que a não-verificação ocorra a partir de um mecanismo MCAR traga resultados satisfatórios em um estudo em que indivíduos com resultados positivos ao teste sob investigação tenham maior probabilidade de ser submetido ao padrão ouro.

\subsection{Métodos Estatísticos nos Estudos em que o Viés de Verificação está Presente}

Begg e Greenes (1983) desenvolveram um método baseado no Teorema de Bayes para a estimação da sensibilidade e da especificidade, entretanto, ele tem como pressuposto que a verificação ou não-verificação de um indivíduo independe do seu real estado (doente ou não doente), ou seja, a decisão de submeter um indivíduo ao padrão ouro, dessa forma, não teria nenhuma associação com a doença. Na prática, esse é um pressuposto muitas vezes inverossímil, dado que, na maioria das vezes, os indivíduos não verificados trazem 
menor predisposição à doença. Para que esse método não traga o viés de verificação para a análise, o tipo de missing teria que ser o da perda ao acaso (MAR), isto é, a verificação pelo padrão ouro depende apenas do resultado do teste e dos fatores de risco observados. Entretanto, a decisão de verificar o indivíduo pode depender de outros fatores relacionados à doença e, conseqüentemente, à doença em si; portanto, a análise será viesada se as perdas forem tratadas como MAR.

Com o objetivo de estimar $S_{E}$ e $S_{P}$, sem a necessidade da suposição utilizada por Begg e Greenes (1983) e considerando que os dados perdidos são não ignoráveis (NI), Zhou (1993) propôs um novo método, introduzindo quatro novas quantidades, denotadas por $\lambda_{11}, \lambda_{10}, \lambda_{01}, \lambda_{00}$ que expressam as probabilidades de um indivíduo ser verificado pelo padrão ouro, em quatro situações distintas: quando o teste é positivo e ele é portador da doença, quando o teste é positivo e ele não é portador da doença, quando o teste é negativo e ele porta a doença e quando o teste é negativo e ele não porta a doença, respectivamente.

Martinez et al. (2006) apresentaram uma metodologia sob uma abordagem bayesiana em que utilizaram a mesma formulação do modelo e função de verossimilhança propostas por Zhou, apenas diferenciando os dois métodos quanto à obtenção dos parâmetros de interesse. Enquanto Zhou utiliza o método de máxima verossimilhança, os autores utilizam a estimação bayesiana.

Diante do problema da não-verificação pelo padrão ouro, algumas técnicas, que serão apresentadas na Seção 2, surgiram para que se tentasse contornar as dificuldades geradas pelo viés de verificação.

Tendo em vista a recente proposta do uso de um método bayesiano, em estudos de dados na presença do viés de verificação, torna-se necessário avaliar o seu desempenho em situações específicas, tais como: efeito do tamanho amostral, da proporção de indivíduos não verificados e da escolha das distribuições a priori.

Assim, no presente trabalho, buscou-se avaliar a eficácia do método bayesiano na estimação da sensibilidade e da especificidade, utilizando-se dados reais e também simulados.

Além disso, foi introduzida uma metodologia, sob enfoque bayesiano, para a estimação 
das medidas de desempenho de dois testes diagnósticos, na presença do viés de verificação, nesse caso, para contornar o problema gerado pelo pressuposto de independência entre $V$ e $D$, admitido por Zhou (1998). 


\section{Avaliação de Testes Diagnósticos na Presença do Viés de Verificação}

\subsection{Notação}

Sejam $\xi$ a prevalência de uma doença em uma população e $D$ o estado da doença, em que $D=1$ (ou simplesmente $D$ ) denota um indivíduo doente, e $D=0$ (ou $\bar{D}$ ), um indivíduo saudável; portanto, $\xi=P(D)$. Seja $T$ uma variável aleatória referente ao resultado do teste diagnóstico, em que $T=1$ (ou somente $T$ ) indica um resultado positivo e, $T=0$ (ou $\bar{T}$ ), indica um resultado negativo. A sensibilidade do teste diagnóstico é dada por $S_{E}=P(T=1 \mid D=1)$, e a especificidade, por $S_{P}=P(T=0 \mid D=0)$. Seja, também, $V$ uma variável aleatória referente à verificação pelo padrão ouro, em que $V=1$ (ou $V$ ) denota um indivíduo verificado, e $V=0$ (ou $\bar{V}$ ), um não verificado. Na Tabela 12 encontram-se as freqüências, quando são cruzadas as variáveis $D, T$ e $V$, considerando-se indivíduos verificados e não verificados.

Tabela 12: Frequiências absolutas para o cruzamento das variáveis $D, T$ e $V$.

\begin{tabular}{|c|c|c|c|c|}
\hline & \multicolumn{2}{|c|}{ Verificados $(V=1)$} & \multirow[t]{2}{*}{ Não verificados $(V=0)$} & \multirow[t]{2}{*}{ Total } \\
\hline & $D=1$ & $D=0$ & & \\
\hline$T=1$ & $s_{1}$ & $r_{1}$ & $u_{1}$ & $n_{1}$ \\
\hline$T=0$ & $s_{2}$ & $r_{2}$ & $u_{2}$ & $n_{2}$ \\
\hline
\end{tabular}

Diante da dificuldade gerada no cálculo das estimativas de sensibilidade e especificidade, quando existe o viés de verificação, alguns autores propuseram metodologias.

\subsection{Método de Begg e Greenes}

Considerando, em seus cálculos, apenas os dados dos pacientes verificados, Begg e Greenes (1983) propuseram estimativas para $S_{E}$ e $S_{P}$, utilizando a fórmula de Bayes. 
Assim, somente assumindo-se independência entre $D$ e $V$, as estimativas propostas podem ser consideradas não viesadas, o que não ocorre na prática. A $S_{E}$ é dada por

$$
P(T \mid D)=\frac{P(T) P(D \mid T V)}{P(T) P(D \mid T V)+P(\bar{T}) P(D \mid \bar{T} V)},
$$

e $S_{P}$ é dada por

$$
P(\bar{T} \mid \bar{D})=\frac{P(\bar{T}) P(\bar{D} \mid \bar{T} V)}{P(\bar{T}) P(\bar{D} \mid \bar{T} V)+P(T) P(\bar{D} \mid T V)}
$$

De (11) e (12), considerando a notação dada pela Tabela 12, tem-se que

$$
\widehat{S_{E}}=\frac{n_{1}\left[s_{1} /\left(s_{1}+r_{1}\right)\right]}{n_{1}\left[s_{1} /\left(s_{1}+r_{1}\right)\right]+n_{2}\left[s_{2} /\left(s_{2}+r_{2}\right)\right]}
$$

e

$$
\widehat{S_{P}}=\frac{n_{2}\left[r_{2} /\left(s_{2}+r_{2}\right)\right]}{n_{1}\left[r_{1} /\left(s_{1}+r_{1}\right)\right]+n_{2}\left[r_{2} /\left(s_{2}+r_{2}\right)\right]} .
$$

Begg e Greenes (1983) também propuseram estimadores para as variâncias de $S_{E}$ e $S_{P}$, obtidas por meio de expansão de séries de Taylor, dados por

$$
\begin{aligned}
& \widehat{\operatorname{var}}\left(\widehat{S_{E}}\right)=\left[\widehat{S_{E}}\left(1-\widehat{S_{E}}\right)\right]^{2}\left[\frac{n}{n_{1} n_{2}}+\frac{r_{1}}{s_{1}\left(s_{1}+r_{1}\right)}+\frac{r_{2}}{s_{2}\left(s_{2}+r_{2}\right)}\right] \\
& \widehat{\operatorname{var}}\left(\widehat{S_{P}}\right)=\left[\widehat{S_{P}}\left(1-\widehat{S_{P}}\right)\right]^{2}\left[\frac{n}{n_{1} n_{2}}+\frac{s_{1}}{r_{1}\left(s_{1}+r_{1}\right)}+\frac{s_{2}}{r_{2}\left(s_{2}+r_{2}\right)}\right] .
\end{aligned}
$$

Assim, intervalos de confiança $(1-\alpha) 100 \%$ para $S_{E}$ e $S_{P}$ são dados, respectivamente, por

$$
\widehat{S_{E}} \pm z_{\alpha / 2} \sqrt{\left[\widehat{S_{E}}\left(1-\widehat{S_{E}}\right)\right]^{2}\left[\frac{n}{n_{1} n_{2}}+\frac{r_{1}}{s_{1}\left(s_{1}+r_{1}\right)}+\frac{r_{2}}{s_{2}\left(s_{2}+r_{2}\right)}\right]}
$$

e

$$
\widehat{S_{P}} \pm z_{\alpha / 2} \sqrt{\left[\widehat{S_{P}}\left(1-\widehat{S_{P}}\right)\right]^{2}\left[\frac{n}{n_{1} n_{2}}+\frac{s_{1}}{r_{1}\left(s_{1}+r_{1}\right)}+\frac{s_{2}}{r_{2}\left(s_{2}+r_{2}\right)}\right]} .
$$

Uma alternativa para a obtenção desses intervalos foi apresentada por Harel e Zhou (2006), baseada na transformação logito de $S_{E}$ e $S_{P}$. Assim, para o caso de $S_{E}$, em vez de se assumir normalidade para $\left(\widehat{S_{E}}-S_{E}\right)$, pode-se sugerir que a transformação logito 
de $S_{E}$ se aproxima mais de uma distribuição normal, tal que $\operatorname{logit}\left(\widehat{S_{E}}\right)-\operatorname{logit}\left(S_{E}\right) \sim$ $N\left(0, \widehat{\operatorname{var}}\left(\operatorname{logit}\left(\widehat{S_{E}}\right)\right)\right)$. Portanto, intervalos de confiança para sensibilidade e especificidade são dados, respectivamente, por

$$
\operatorname{logit}^{-1}\left[\operatorname{logit}\left(\widehat{S_{E}}\right)-z_{\alpha / 2} \sqrt{\widehat{\operatorname{var}}\left(\operatorname{logit}\left(\widehat{S_{E}}\right)\right)} ; ; \operatorname{logit}{ }^{-1}\left[\operatorname{logit}\left(\widehat{S_{E}}\right)+z_{\alpha / 2} \sqrt{\widehat{\operatorname{var}}\left(\operatorname{logit}\left(\widehat{S_{E}}\right)\right)}\right]\right.
$$

e

$$
\operatorname{logit}^{-1}\left[\operatorname{logit}\left(\widehat{S_{P}}\right)-z_{\alpha / 2} \sqrt{\widehat{\operatorname{var}}\left(\operatorname{logit}\left(\widehat{S_{P}}\right)\right)} ; \operatorname{logit}^{-1}\left[\operatorname{logit}\left(\widehat{S_{P}}\right)+z_{\alpha / 2} \sqrt{\widehat{\operatorname{var}}\left(\operatorname{logit}\left(\widehat{S_{P}}\right)\right)}\right]\right.
$$

em que $\operatorname{logit}(\pi)=\log (\pi /(1-\pi)), z_{\alpha / 2}$ é o percentil da distribuição normal padrão e

$$
\widehat{\operatorname{var}}\left(\operatorname{logit}\left(\widehat{S_{E}}\right)\right)=\left[\frac{n}{n_{1} n_{2}}+\frac{r_{1}}{s_{1}\left(s_{1}+r_{1}\right)}+\frac{r_{2}}{s_{2}\left(s_{2}+r_{2}\right)}\right]
$$

e

$$
\widehat{\operatorname{var}}\left(\operatorname{logit}\left(\widehat{S_{P}}\right)\right)=\left[\frac{n}{n_{1} n_{2}}+\frac{s_{1}}{r_{1}\left(s_{1}+r_{1}\right)}+\frac{s_{2}}{r_{2}\left(s_{2}+r_{2}\right)}\right]
$$

Em um estudo de simulação via Monte Carlo, Harel e Zhou (2006) mostraram que a probabilidade de cobertura dos intervalos de confiança dados em (19) e (20) é mais próxima da nominal do que a obtida a partir dos IC dados em (17) e (18).

\subsection{Método de Zhou}

Considerando-se os dados da Tabela 12 como uma amostra de uma distribuição multinomial, na Tabela 13, a seguir, encontram-se as probabilidades associadas às combinações entre as variáveis $V, D$ e $T$, em que $\lambda_{11}=P(V \mid T D), \lambda_{01}=P(V \mid T \bar{D}), \lambda_{10}=P(V \mid \bar{T} D)$ e $\lambda_{00}=P(V \mid \overline{T D})$. 
Tabela 13: Probabilidades para o cruzamento das variáveis $D, T$ e $V$.

\begin{tabular}{ccccc}
\hline & \multicolumn{2}{c}{ Verificados $(V=1)$} & Não verificados $(V=0)$ & Total \\
\cline { 2 - 3 } & $D=1$ & $D=0$ & & \\
\hline$T=1$ & $\lambda_{11} S_{E} \xi$ & $\lambda_{01}\left(1-S_{P}\right)(1-\xi)$ & $\left(1-\lambda_{11}\right) S_{E} \xi+\left(1-\lambda_{01}\right)\left(1-S_{P}\right)(1-\xi)$ & $n_{1}$ \\
$T=0$ & $\lambda_{10}\left(1-S_{E}\right) \xi$ & $\lambda_{00} S_{P}(1-\xi)$ & $\left(1-\lambda_{10}\right)\left(1-S_{E}\right) \xi+\left(1-\lambda_{00}\right)(1-\xi) S_{P}$ & $n_{2}$ \\
\hline
\end{tabular}

No cálculo das probabilidades dadas na Tabela 13 , vale obsevar que $P(T=1, D=$ $1, V=1)=P(T D V)=P(D) P(V \mid T D) P(T \mid D)=\lambda_{11} S_{E} \xi$. Também, $P(T=1, V=$ $0)=P(T \bar{V})=P(D)[1-P(V \mid T D)] P(T \mid D)+[1-P(D)][1-P(V \mid T \bar{D})] P(\bar{T} \mid \bar{D})=(1-$ $\left.\lambda_{11}\right) S_{E} \xi+\left(1-\lambda_{01}\right)\left(1-S_{P}\right)(1-\xi)$. Da mesma maneira, podem-se encontrar as outras probabilidades. Do modelo de probabilidade dado na Tabela 13 e, considerando-se os dados $\mathcal{D}=\left\{\left(d_{i}, t_{i}, v_{i}\right) ; i=1, \ldots, n\right\}$, em que $d_{i}=1$ para $D$ e 0 para $\bar{D}, t_{i}=1$ para $T$ e 0 para $\bar{T}, v_{i}=1$ para $V$ e 0 para $\bar{V}$ e ainda, assumindo, para os indivíduos verificados, que $s_{1}=\sum_{i=1}^{n} t_{i} d_{i}, s_{2}=\sum_{i=1}^{n}\left(1-t_{i}\right) d_{i}, r_{1}=\sum_{i=1}^{n} t_{i}\left(1-d_{i}\right), r_{2}=\sum_{i=1}^{n}\left(1-t_{i}\right)\left(1-d_{i}\right)$ e, para os não verificados, $u_{1}=\sum_{i=1}^{n} t_{i} v_{i}, u_{2}=\sum_{i=1}^{n}\left(1-t_{i}\right)\left(1-v_{i}\right)$, a função de verossimilhança para $\boldsymbol{\theta}_{1}=\left(\lambda_{11}, \lambda_{10}, \lambda_{01}, \lambda_{00}, S_{E}, S_{P}, \xi\right)^{\prime}$ é dada por:

$$
\begin{aligned}
L\left(\boldsymbol{\theta}_{1}\right)= & \xi^{\left(s_{1}+s_{2}\right)}(1-\xi)^{\left(r_{1}+r_{2}\right)}\left(\lambda_{11} S_{E}\right)^{s_{1}}\left[\lambda_{01}\left(1-S_{P}\right)\right]^{r_{1}}\left[\lambda_{10}\left(1-S_{E}\right)\right]^{s_{2}}\left[\lambda_{00} S_{P}\right]^{r_{2}} \\
& {\left[\left(1-\lambda_{11}\right) S_{E} \xi+\left(1-\lambda_{01}\right)\left(1-S_{P}\right)(1-\xi)\right]^{u_{1}}\left[\left(1-\lambda_{10}\right)\left(1-S_{E}\right)+\left(1-\lambda_{00}\right)(1-\xi)\right]^{u_{2}} . }
\end{aligned}
$$

Tomando-se o logaritmo dessa expressão, tem-se

$$
\begin{aligned}
\log \left(L\left(\boldsymbol{\theta}_{1}\right)\right)= & \left(s_{1}+s_{2}\right) \log \xi+\left(r_{1}+r_{2}\right) \log (1-\xi)+s_{1} \log \lambda_{11}+s_{1} \log S_{E}+r_{1} \log \lambda_{01}+ \\
& +r_{1} \log \left(1-S_{P}\right)+r_{1} \log \left(1-S_{P}\right)+s_{2} \log \lambda_{10}+s_{2} \log \lambda_{10}+s_{2} \log \left(1-S_{E}\right) \\
& +r_{2} \log \lambda_{00}+r_{2} \log S_{P}+u_{1} \log \left[\left(1-\lambda_{11}\right) S_{E} \xi+\left(1-\lambda_{01}\right)\left(1-S_{P}\right)(1-\xi)\right] \\
& +u_{2} \log \left[\left(1-\lambda_{10}\right)\left(1-S_{E}\right) \xi+\left(1-\lambda_{00}\right) S_{P}(1-\xi)\right] .
\end{aligned}
$$

Aqui, para contornar um problema de identificabilidade (há mais parâmetros desconhecidos do que variáveis), na estimação de $S_{E}, S_{P}, \xi, \lambda_{11}, \lambda_{10}, \lambda_{01}$ e $\lambda_{00}$, Zhou (1993) 
admitiu as razões $k_{1}=\frac{\lambda_{11}}{\lambda_{01}}$ e $k_{2}=\frac{\lambda_{10}}{\lambda_{00}}$, com $k_{1}$ e $k_{2}$ sendo constantes conhecidas. Então,

$$
\begin{aligned}
\log \left(L\left(\boldsymbol{\theta}_{1}\right)\right)= & \left(s_{1}+s_{2}\right) \log \xi+\left(r_{1}+r_{2}\right) \log (1-\xi)+s_{1} \log S_{E}+r_{1} \log \left(1-S_{P}\right) \\
& +s_{2} \log \left(1-S_{E}\right)+r_{2} \log S_{P}+u_{1} \log \left[\left(1-\lambda_{11}\right) S_{E} \xi\right. \\
& \left.+\left(1-\frac{\lambda_{11}}{k_{1}}\right)\left(1-S_{P}\right)(1-\xi)\right]+s_{1} \log \left(\lambda_{11}\right)+r_{1} \log \left(\frac{\lambda_{11}}{k_{1}}\right) \\
& +s_{2} \log \left(\lambda_{10}\right)+r_{2} \log \left(\frac{\lambda_{10}}{k_{2}}\right)+u_{2} \log \left[\left(1-\lambda_{10}\right)\left(1-S_{E}\right) \xi\right. \\
& \left.+\left(1-\frac{\lambda_{10}}{k_{2}}\right) S_{P}(1-\xi)\right] .
\end{aligned}
$$

Após algumas simplificações, a função log-verosimilhança é dada por:

$$
\begin{aligned}
\log \left(L\left(\boldsymbol{\theta}_{1}\right)\right)= & \left(s_{1}+s_{2}\right) \log \xi+\left(r_{1}+r_{2}\right) \log (1-\xi)+s_{1} \log S_{E}+r_{1} \log \left(1-S_{P}\right) \\
& +s_{2} \log \left(1-S_{E}\right)+r_{2} \log S_{P}+\left(s_{1}+r_{1}\right) \log \lambda_{11}+\left(s_{2}+r_{2}\right) \log \lambda_{10} \\
& -\left(r_{1}+u_{1}\right) \log k_{1}-\left(r_{2}+u_{2}\right) \log k_{2}+u_{1} \log \left[k_{1}\left(1-\lambda_{11}\right) S_{E} \xi\right. \\
& \left.+\left(k_{1}-\lambda_{11}\right)\left(1-S_{P}\right)(1-\xi)\right]+u_{2} \log \left[k_{2}\left(1-\lambda_{10}\right)\left(1-S_{E}\right) \xi\right. \\
& \left.+\left(k_{2}-\lambda_{10}\right) S_{P}(1-\xi)\right] .
\end{aligned}
$$

Nota-se, agora, que existem apenas cinco parâmetros a serem estimados. Então, assumindo-se que $k_{1}$ e $k_{2}$ são constantes conhecidas, os estimadores para $S_{E}, S_{P}, \xi, \lambda_{11}$ e $\lambda_{10}$ são as soluções do sistema de equações dado a seguir.

$$
\begin{aligned}
0= & \frac{s_{1}}{S_{E}}-\frac{s_{2}}{1-S_{E}}+\frac{k_{1}\left(1-\lambda_{11}\right) \xi}{k_{1}\left(1-\lambda_{11}\right) S_{E} \xi+\left(k_{1}-\lambda_{11}\right)\left(1-S_{P}\right)(1-\xi)} u_{1} \\
& -\frac{k_{2}\left(1-\lambda_{10}\right) \xi}{k_{2}\left(1-\lambda_{10}\right)\left(1-S_{E}\right) \xi+\left(k_{1}-\lambda_{10}\right) S_{P}(1-\xi)} u_{2} ; \\
0= & -\frac{r_{1}}{1-S_{P}}+\frac{r_{2}}{S_{P}}-\frac{\left(k_{1}-\lambda_{11}\right)(1-\xi)}{k_{1}\left(1-\lambda_{11}\right) S_{E} \xi+\left(k_{1}-\lambda_{11}\right)\left(1-S_{P}\right)(1-\xi)} u_{1} \\
& +\frac{\left(k_{2}-\lambda_{10}\right)(1-\xi)}{k_{2}\left(1-\lambda_{10}\right)\left(1-S_{E}\right) \xi+\left(k_{1}-\lambda_{10}\right) S_{P}(1-\xi)} u_{2} ; \\
0 & \frac{s_{1}+s_{2}}{\xi}-\frac{r_{1}+r_{2}}{1-\xi}+\frac{k_{1}\left(1-\lambda_{11}\right) S_{E}-\left(k_{1}-\lambda_{11}\right)\left(1-S_{P}\right)}{k_{1}\left(1-\lambda_{11}\right) S_{E} \xi+\left(k_{1}-\lambda_{11}\right)\left(1-S_{P}\right)(1-\xi)} u_{1} \\
& +\frac{k_{2}\left(1-\lambda_{10}\right)\left(1-S_{E}\right)-\left(k_{2}-\lambda_{10}\right) S_{P}}{k_{2}\left(1-\lambda_{10}\right)\left(1-S_{E}\right) \xi+\left(k_{1}-\lambda_{11}\right)\left(1-S_{P}\right)(1-\xi)} u_{2} ;
\end{aligned}
$$




$$
\begin{aligned}
0 & =\frac{s_{1}+r_{1}}{\lambda_{11}}-\frac{k_{1} S_{E} \xi+\left(1-S_{P}\right)(1-\xi)}{k_{1}\left(1-\lambda_{11}\right) S_{E} \xi+\left(k_{1}-\lambda_{11}\right)\left(1-S_{P}\right)(1-\xi)} u_{1} ; \\
0 & =\frac{r_{2}+s_{2}}{\lambda_{10}}-\frac{k_{2}\left(1-S_{E}\right) \xi+S_{P}(1-\xi)}{k_{2}\left(1-\lambda_{10}\right)\left(1-S_{E}\right) \xi+\left(k_{1}-\lambda_{10}\right) S_{P}(1-\xi)} u_{2} .
\end{aligned}
$$

Essas expressões são obtidas a partir da derivação de (24) com relação a cada parâmetro de interesse.

Obter as soluções para $S_{E}, S_{P}, \xi, \lambda_{11}$ e $\lambda_{10}$, a partir desse sistema, parece difícil; então, uma aproximação, dada a seguir, permite que se obtenham expressões explícitas para os estimadores de máxima verossimilhança.

Seja \# $(A)$ o número total de elementos que satisfazem as condições em $A$. Para se estimarem $S_{E}=P(T \mid D)=\#(T D) / \#(D)$ e $S_{P}=P(\bar{T} \mid \bar{D})=\#(\overline{T D}) / \#(\bar{D})$, é necessário obter o número total de pacientes e o total de pacientes doentes, os quais apresentam resultado positivo para o padrão ouro. Então, tem-se que

$$
P(V \mid T D)=\frac{s_{1}}{\#(D T)}, P(V \mid T \bar{D})=\frac{r_{1}}{\#(\bar{D} T)}
$$

e

$$
P(V \mid \bar{T} D)=\frac{s_{2}}{\#(D \bar{T})}, P(V \mid \overline{T D})=\frac{r_{2}}{\#(\overline{D T})}
$$

Agora, sendo $k_{1}=\frac{P(V \mid T D)}{P(V \mid T \bar{D})}$, então

$$
\begin{aligned}
k_{1} & =\frac{\frac{s_{1}}{\#(T D)}}{\frac{r_{1}}{\#(\bar{D})}}=\frac{s_{1} \#(T \bar{D})}{r_{1} \#(T D)} \text { e, conseqüentemente, } \\
\#(T D) & =\frac{\#(T \bar{D}) s_{1}}{k_{1} r_{1}}=\frac{s_{1}}{k_{1} r_{1}}\left[s_{1}+r_{1}+u_{1}-\#(T D)\right] .
\end{aligned}
$$

Tem-se, assim,

$$
\begin{aligned}
k_{1} r_{1} & =s_{1}\left[\frac{s_{1}+r_{1}+u_{1}-\#(T D)}{\#(T D)}\right], \text { portanto } \\
\#(T D) & =\frac{s_{1} n_{1}}{k_{1} r_{1}+s_{1}} .
\end{aligned}
$$


Analogamente, sendo $k_{2}=\frac{P(V \mid \bar{T} D)}{P(V \mid \bar{T} D)}$, tem-se

$$
\begin{aligned}
k_{2} & =\frac{\frac{s_{2}}{\#(\bar{T} D)}}{\frac{r_{2}}{\#(\overline{T D})}}=\frac{s_{2} \#(\overline{T D})}{r_{2} \#(\bar{T} D)}, \text { o que leva à expressão } \\
\#(\bar{T} D) & =\frac{\#(\overline{T D}) s_{2}}{k_{2} r_{2}} .
\end{aligned}
$$

Assim

$$
\begin{aligned}
{\left[s_{2}+r_{2}+u_{2}-\#(\overline{T D})\right] } & =\frac{s_{2}}{k_{2} r_{2}} \#(\overline{T D}) \mathrm{e} \\
k_{2} r_{2} n_{2}-k_{2} r_{2} \#(\overline{T D}) & =s_{2} \#(\overline{T D}), \text { o que leva ao resultado } \\
\#(\overline{T D}) & =\frac{k_{2} r_{2} n_{2}}{k_{2} r_{2}+s_{2}},
\end{aligned}
$$

em que $\#(T \bar{D})=n_{1}-\#(T D), \#(\bar{T} D)=n_{2}-\#(\overline{T D}), n_{1}=s_{1}+r_{1}+u_{1}$ e $n_{2}=s_{2}+r_{2}+u_{2}$.

Portanto, $\#(D)=\frac{s_{1} n_{1}}{k_{1} r_{1}+s_{1}}+\frac{s_{2} n_{2}}{k_{2} r_{2}+s_{2}}$ e $\#(\bar{D})=\frac{k_{1} r_{1} n_{1}}{k_{1} r_{1}+s_{1}}+\frac{k_{2} r_{2} n_{2}}{k_{2} r_{2}+s_{2}}$

Agora, de uma maneira intuitiva, os estimadores de $S_{E}, S_{P}, \xi, \lambda_{11}$ e $\lambda_{10}$ são dados por:

$$
\begin{aligned}
\widehat{S_{E}} & =\frac{\left(s_{1} n_{1}\right) /\left(k_{1} r_{1}+s_{1}\right)}{\left(s_{1} n_{1}\right) /\left(k_{1} r_{1}+s_{1}\right)+\left(s_{2} n_{2}\right) /\left(k_{2} r_{2}+s_{2}\right)} ; \\
\widehat{S_{P}} & =\frac{\left(k_{2} r_{2} n_{2}\right) /\left(k_{2} r_{2}+s_{2}\right)}{\left(k_{1} r_{1} n_{1}\right) /\left(k_{1} r_{1}+s_{1}\right)+\left(k_{2} r_{2} n_{2}\right) /\left(k_{2} r_{2}+s_{2}\right)} \\
\widehat{\xi} & =\frac{\left(s_{1} n_{1}\right) /\left(k_{1} r_{1}+s_{1}\right)+\left(s_{2} n_{2}\right) /\left(k_{2} r_{2}+s_{2}\right)}{n} \\
\widehat{\lambda_{11}} & =\frac{k_{1} r_{1}+s_{1}}{n_{1}} \text { e } \widehat{\lambda_{10}}=\frac{k_{2} r_{2}+s_{2}}{n_{2}}
\end{aligned}
$$

em que $n=n_{1}+n_{2}$.

Uma prova de que esses estimadores são de máxima verossimilhança encontra-se em Zhou (1993).

Se os valores de $k_{1}$ e $k_{2}$ são conhecidos, os estimadores de máxima verossimilhança para $S_{E}, S_{P}, \xi, \lambda_{11}$ e $\lambda_{10}$ são dados por $\widehat{S_{E}}, \widehat{S_{P}}, \widehat{\xi}, \widehat{\lambda_{11}}$ e $\widehat{\lambda_{10}}$, como definido anteriormente. 
Logo,

$$
\sqrt{n}\left(\begin{array}{c}
\widehat{S_{E}}-S_{E} \\
\widehat{S_{P}}-S_{P} \\
\widehat{\xi}-\xi \\
\widehat{\lambda_{11}}-\lambda_{11} \\
\widehat{\lambda_{10}}-\lambda_{10}
\end{array}\right) \longrightarrow N_{M}\left(\left(\begin{array}{l}
0 \\
0 \\
0 \\
0 \\
0
\end{array}\right), \Sigma^{-1}\right)
$$

em que $N_{M}$ representa uma distribuição normal multivariada. Os elementos da matriz de covariância $\Sigma$, obtidos a partir da matriz de informação de Fisher, são dados por:

$$
\begin{gathered}
\sigma_{11}=\frac{\lambda_{11} \xi}{S_{E}}+\frac{\lambda_{10} \xi}{1-S_{E}}+\frac{\left(1-\lambda_{11}\right)^{2} \xi^{2}}{\left(1-\lambda_{11}\right) S_{E} \xi+\left(1-\lambda_{11} / k_{1}\right)\left(1-S_{P}\right)(1-\xi)}+ \\
+\frac{\left(1-\lambda_{10}\right)^{2} \xi^{2}}{\left(1-\lambda_{10}\right)\left(1-S_{P}\right) \xi+\left(1-\lambda_{10} / k_{2}\right) S_{P}(1-\xi)}
\end{gathered}
$$




$$
\begin{aligned}
& \sigma_{13}= \frac{\left(1-\lambda_{11} / k_{1}\right)\left(1-\lambda_{11}\right)\left(1-S_{P}\right)}{\left(1-\lambda_{10}\right) S_{E} \xi+\left(1-\lambda_{11} / k_{1}\right)\left(1-S_{P}\right)(1-\xi)}+ \\
&+\frac{\left(1-\lambda_{10} / k_{2}\right)\left(1-\lambda_{10}\right) S_{P}}{\left(1-\lambda_{10}\right)\left(1-S_{P}\right) \xi+\left(1-\lambda_{10} / k_{2}\right) S_{P}(1-\xi)} ; \\
& \sigma_{14}= \frac{\left(1-1 / k_{1}\right) \xi(1-\xi)\left(1-S_{P}\right)}{\left(1-\lambda_{11}\right) S_{E} \xi+\left(1-\lambda_{11} / k_{1}\right)\left(1-S_{P}\right)(1-\xi)} \\
& \sigma_{15}= \frac{\left(1-1 / k_{2}\right) \xi(1-\xi) S_{P}}{\left(1-\lambda_{10}\right)\left(1-S_{E}\right) \xi+\left(1-\lambda_{10} / k_{2}\right) S_{P}(1-\xi)} ; \\
& \sigma_{23}= \frac{\left(1-\lambda_{11} / k_{1}\right)\left(1-\lambda_{11}\right) S_{E}}{\left(1-\lambda_{11}\right) S_{E} \xi+\left(1-\lambda_{11} / k_{1}\right)\left(1-S_{P}\right)(1-\xi)}+ \\
&+\frac{\left(1-\lambda_{10} / k_{2}\right)\left(1-\lambda_{10}\right)\left(1-S_{E}\right)}{\left(1-\lambda_{10}\right)\left(1-S_{P}\right) \xi+\left(1-\lambda_{10} / k_{2}\right) S_{P}(1-\xi)} ; \\
& \sigma_{35}=\frac{\left(1-1 / k_{1}\right) \xi(1-\xi) S_{E}}{\left(1-\lambda_{10}\right)\left(1-S_{E}\right) \xi+\left(1-\lambda_{10} / k_{2}\right) S_{P}(1-\xi)} ; \sigma_{45}
\end{aligned}=0 .
$$

Como já foi dito, podem-se obter os estimadores de máxima verossimilhança para $S_{E}$, $S_{P}, \xi, \lambda_{11}$ e $\lambda_{10}$, considerando-se que $k_{1}$ e $k_{2}$ são conhecidos. Assumindo-se que, dentre os $u_{1}$ pacientes com resultado positivo para o teste, $s_{11}$ realmente estão doentes, e, dentre os $u_{2}$ com teste negativo, $s_{12}$ realmente estão doentes, as expressões para se encontrarem valores plausíveis para $k_{1}$ e $k_{2}$ são:

$$
k_{1}=\frac{P(V \mid T D)}{P(V \mid T \bar{D})}=\frac{P(V T D) / P(T D)}{P(V T \bar{D}) / P(T \bar{D})}=\frac{s_{1} /\left(s_{1}+s_{11}\right)}{r_{1} /\left(r_{1}+u_{1}-s_{11}\right)}=\frac{s_{1}\left(r_{1}+u_{1}-s_{11}\right)}{r_{1}\left(s_{1}+s_{11}\right)}
$$

e

$$
k_{2}=\frac{P(V \mid \bar{T} D)}{P(V \mid \overline{T D})}=\frac{P(V \bar{T} D) / P(\bar{T} D)}{P(V \overline{T D}) / P(\overline{T D})}=\frac{s_{2} /\left(s_{2}+s_{12}\right)}{r_{2} /\left(r_{2}+u_{2}-s_{12}\right)}=\frac{s_{2}\left(r_{2}+u_{2}-s_{12}\right)}{r_{2}\left(s_{2}+s_{12}\right)} \text {. }
$$


Na prática, apenas se sabe que $0 \leq s_{11} \leq u_{1}$ e $0 \leq s_{12} \leq u_{2}$. Portanto, os limites para os possíveis valores de $k_{1}$ e $k_{2}$ são dados por:

$$
\frac{s_{1}}{s_{1}+u_{1}} \leq k_{1} \leq \frac{r_{1}+u_{1}}{r_{1}} \text { e } \frac{s_{2}}{s_{2}+u_{2}} \leq k_{2} \leq \frac{r_{2}+u_{2}}{r_{2}}
$$

A partir dessas equações, nota-se que, se $u_{1}$ e $u_{2}$ são pequenos, em relação a $s_{1}$ e $s_{2}$, os valores de $k_{1}$ e $k_{2}$ são, aproximadamente, iguais a 1 . Nesse caso, os estimadores propostos por Begg e Greenes (1983) podem ser usados para se estimarem a sensibilidade e a especificidade. Entretanto, se o número de pacientes não verificados ( $u_{1}$ e $\left.u_{2}\right)$ é grande, com relação a $s_{1}$ e $s_{2}$, os limites dos possíveis valores para $k_{1}$ e $k_{2}$ também são grandes.

Harel e Zhou (2006) apresentaram uma nova metodologia em que foi utilizada a imputação múltipla para estimar a sensibilidade e a especificidade, na presença do viés de verificação. Entretanto, Hanley et al. (2007), em uma carta ao editor, apontaram um grande número de erros computacionais cometidos pelos autores, que em resposta às críticas, assumiram alguns dos erros, retificando a eficiência do método na estimação das medidas de desempenho de testes diagnósticos (ver HAREL e ZHOU, 2007).

\subsection{Exemplos}

Considerando-se os dados do Exemplo 1, foram simuladas algumas situações para indivíduos não verificados. Utilizando-se os métodos de Begg e Greenes (1983) e Harel e Zhou (2006), já apresentados, algumas simulações (com variações para as probabilidades $\lambda_{11}, \lambda_{10}, \lambda_{01}$ e $\left.\lambda_{00}\right)$ foram feitas para estimação da sensibilidade, especificidade e seus intervalos de confiança. O painel (b) da Tabela 14, por exemplo, representa a situação em que foram retirados $20 \%$ dos indivíduos de cada célula da Tabela 2 (dados completos painel $(a)$ ). A proporção retirada foi considerada como sendo de indivíduos não verificados. Assim, as freqüências, nesse caso, seriam dadas por $s_{1}=652 ; r_{1}=92 ; u_{1}=186 ; s_{2}=166$; $r_{2}=262 ; u_{2}=107$. O painel $(e)$ representa a situação em que foram retirados $50 \%$ dos indivíduos que tiveram resultado positivo para o teste e para o padrão ouro e também $50 \%$ daqueles que tiveram resultado negativo para o teste e para o padrão ouro. Assim, 
nesse caso, as freqüências seriam dadas por $s_{1}=408 ; r_{1}=115 ; u_{1}=407 ; s_{2}=208$; $r_{2}=164 ; u_{2}=163$. Esse mesmo procedimento foi aplicado para as outras simulações. Os resultados obtidos estão na Tabela 14. É importante lembrar que os erros padrão para o logit B\&G são os erros do logito do estimador, obtidos a partir das expressões (15) e (16).

Tabela 14: Estimativas de sensibilidade e especificidade - Método de Begg e Greenes.

\begin{tabular}{|c|c|c|c|c|c|c|}
\hline \multirow[t]{2}{*}{ Painel } & \multirow{2}{*}{$\begin{array}{c}\text { Resultado } \\
\text { do Teste }\end{array}$} & \multicolumn{2}{|c|}{ Verificados pelo P. O. } & \multirow{2}{*}{$\begin{array}{c}\text { Não } \\
\text { Verificados }\end{array}$} & \multirow{2}{*}{$\begin{array}{c}\text { Estimativas } \\
\text { de B\&G }(\%)\end{array}$} & \multirow{2}{*}{$\begin{array}{c}\text { Estimativas } \\
\text { Logit B\&G }(\%)\end{array}$} \\
\hline & & Positivo & Negativo & & & \\
\hline (a) $\lambda_{11}=\lambda_{00}=100 \%$ & Positivo & 815 & 115 & - & $\widehat{S_{E}}=79,67(76,90 ; 82,43)$ & $\widehat{S_{E}}=79,67(77,08 ; 82,02)$ \\
\hline$\lambda_{01}=\lambda_{10}=100 \%$ & Negativo & 208 & 327 & - & $\widehat{S_{P}}=73,98(69,23 ; 78,74)$ & $\widehat{S_{P}}=73,98(69,69 ; 77,86)$ \\
\hline (b) $\lambda_{11}=\lambda_{00}=80 \%$ & Positivo & 652 & 92 & 186 & $\widehat{S_{E}}=79,70(76,81 ; 82,60)$ & $\widehat{S_{E}}=79,70(77,04 ; 82,21)$ \\
\hline$\lambda_{01}=\lambda_{10}=80 \%$ & Negativo & 166 & 262 & 107 & $\widehat{S_{P}}=74,08(68,81 ; 79,24)$ & $\widehat{S_{P}}=74,08(69,32 ; 78,27)$ \\
\hline (c) $\lambda_{11}=\lambda_{00}=50 \%$ & Positivo & 408 & 58 & 464 & $\widehat{S_{E}}=79,70(76,34 ; 83,12)$ & $\widehat{S_{E}}=79,70(76,53 ; 82,56)$ \\
\hline$\lambda_{01}=\lambda_{10}=50 \%$ & Negativo & 104 & 164 & 267 & $\widehat{S_{P}}=73,90(67,64 ; 80,21)$ & $\widehat{S_{P}}=73,90(68,10 ; 78,93)$ \\
\hline (d) $\lambda_{11}=50 \%$ & Positivo & 408 & 115 & 407 & $\widehat{S_{E}}=77,72(74,65 ; 80,84)$ & $\widehat{S_{E}}=77,72(74,92 ; 80,36)$ \\
\hline$\lambda_{00}=\lambda_{01}=\lambda_{10}=100 \%$ & Negativo & 208 & 327 & 0 & $\widehat{S_{P}}=61,57(55,31 ; 67,75)$ & $\widehat{S_{P}}=61,57(56,63 ; 66,25)$ \\
\hline (e) $\lambda_{11}=\lambda_{00}=50 \%$ & Positivo & 408 & 115 & 407 & $\widehat{S_{E}}=70,84(67,23 ; 74,48)$ & $\widehat{S_{E}}=70,84(67,71 ; 73,76)$ \\
\hline$\lambda_{01}=\lambda_{10}=100 \%$ & Negativo & 208 & 164 & 163 & $\widehat{S_{P}}=53,62(45,92 ; 61,23)$ & $\widehat{S_{P}}=53,62(48,01 ; 59,13)$ \\
\hline (f) $\lambda_{11}=\lambda_{00}=\lambda_{01}=100 \%$ & Positivo & 815 & 115 & 0 & $\widehat{S_{E}}=86,34(83,86 ; 88,94)$ & $\widehat{S_{E}}=86,34(83,88 ; 88,52)$ \\
\hline$\lambda_{10}=50 \%$ & Negativo & 104 & 327 & 104 & $\widehat{S_{P}}=77,92(73,94 ; 82,03)$ & $\widehat{S_{P}}=77,92(74,12 ; 81,35)$ \\
\hline (g) $\lambda_{11}=\lambda_{00}=\lambda_{10}=100 \%$ & Positivo & 815 & 58 & 57 & $\widehat{S_{E}}=80,71(78,05 ; 83,34)$ & $\widehat{S_{E}}=80,71(78,24 ; 82,93)$ \\
\hline$\lambda_{01}=50 \%$ & Negativo & 208 & 327 & 0 & $\widehat{S_{P}}=84,17(80,02 ; 88,25)$ & $\widehat{S_{P}}=84,17(80,03 ; 87,54)$ \\
\hline
\end{tabular}

Nota-se que, nos painéis $(a)$ e $(b)$, os valores da sensibilidade e especificidade são praticamente iguais aos calculados quando todos os indivíduos são verificados $\left(\widehat{S_{E}}=\right.$ $79,71 \%$ e $\widehat{S_{P}}=74,02 \%$ ). Isso ocorre porque o método proposto por Begg e Greenes é eficiente quando se tem $k_{1}=k_{2}=1$, ou seja, $k_{1}=\frac{\lambda_{11}}{\lambda_{01}}$ e $k_{2}=\frac{\lambda_{10}}{\lambda_{00}}$. Pode-se verificar, também, que à medida que a proporção de indivíduos não verificados aumenta, os erros padrão das estimativas sofrem um suave aumento. Já nas situações $(d),(e),(f)$ e $(g)$, as estimativas começam a sofrer alterações, dependendo da proporção retirada dos indivíduos verificados. Esses resultados são esperados, pois agora não se tem mais que $k_{1}=k_{2}=1$, e o método de Begg e Greenes mostra-se limitado para a estimação de sensibilidade e especifidade. Utilizando-se a metodologia proposta por Zhou, as mesmas situações foram consideradas e, calculados os limites para $k_{1}$ e $k_{2}$, a partir de (32), foram obtidos os seguintes limites para a sensibilidade e para a especificidade, na Tabela 15. 
Tabela 15: Limites para sensibilidade e especificidade - Método de Zhou.

\begin{tabular}{|c|c|c|c|c|c|c|}
\hline \multirow[t]{2}{*}{ Painel } & \multirow{2}{*}{$\begin{array}{c}\text { Resultado } \\
\text { do Teste }\end{array}$} & \multicolumn{2}{|c|}{ Verificados pelo P. O. } & \multirow{2}{*}{$\begin{array}{c}\text { Não } \\
\text { Verificados }\end{array}$} & \multirow{2}{*}{$\begin{array}{c}\text { Limites } \\
\text { Método de Zhou (\%) }\end{array}$} & \multirow{2}{*}{$\begin{array}{l}\text { Estimativas } \\
\text { de B\&G }(\%)\end{array}$} \\
\hline & & Positivo & Negativo & & & \\
\hline (a) $\lambda_{11}=\lambda_{00}=100 \%$ & Positivo & 815 & 115 & - & - & $\widehat{S_{E}}=79,67(76,90 ; 82,43)$ \\
\hline$\lambda_{01}=\lambda_{10}=100 \%$ & Negativo & 208 & 327 & - & - & $\widehat{S_{P}}=73,98(69,23 ; 78,74)$ \\
\hline (b) $\lambda_{11}=\lambda_{00}=80 \%$ & Positivo & 652 & 92 & 186 & $70,53<S_{E}<83,51$ & $\widehat{S_{E}}=79,70(76,81 ; 82,60)$ \\
\hline$\lambda_{01}=\lambda_{10}=80 \%$ & Negativo & 166 & 262 & 107 & $48,57<S_{P}<80,04$ & $\widehat{S_{P}}=74,08(68,81 ; 79,24)$ \\
\hline (c) $\lambda_{11}=\lambda_{00}=50 \%$ & Positivo & 408 & 58 & 464 & $52,43<S_{E}<89,35$ & $\widehat{S_{E}}=79,70(76,34 ; 83,12)$ \\
\hline$\lambda_{01}=\lambda_{10}=50 \%$ & Negativo & 104 & 164 & 267 & $23,94<S_{P}<88,10$ & $\widehat{S_{P}}=73,90(67,64 ; 80,21)$ \\
\hline (d) $\lambda_{11}=50 \%$ & Positivo & 408 & 115 & 407 & $66,23<S_{E}<79,72$ & $\widehat{S_{E}}=77,72(74,65 ; 80,84)$ \\
\hline$\lambda_{00}=\lambda_{01}=\lambda_{10}=100 \%$ & Negativo & 208 & 327 & 0 & $38,51<S_{P}<74,02$ & $\widehat{S_{P}}=61,57(55,31 ; 67,75)$ \\
\hline (e) $\lambda_{11}=\lambda_{00}=50 \%$ & Positivo & 408 & 115 & 407 & $52,49<S_{E}<79,75$ & $\widehat{S_{E}}=70,84(67,23 ; 74,48)$ \\
\hline$\lambda_{01}=\lambda_{10}=100 \%$ & Negativo & 208 & 164 & 163 & $23,94<S_{P}<74,03$ & $\widehat{S_{P}}=53,62(45,92 ; 61,23)$ \\
\hline (f) $\lambda_{11}=\lambda_{00}=\lambda_{01}=100 \%$ & Positivo & 815 & 115 & 0 & $79,72<S_{E}<88,73$ & $\widehat{S_{E}}=86,34(83,86 ; 88,94)$ \\
\hline$\lambda_{10}=50 \%$ & Negativo & 104 & 327 & 104 & $74,02<S_{P}<78,96$ & $\widehat{S_{P}}=77,92(73,94 ; 82,03)$ \\
\hline (g) $\lambda_{11}=\lambda_{00}=\lambda_{10}=100 \%$ & Positivo & 815 & 58 & 57 & $79,78<S_{E}<80,71$ & $\widehat{S_{E}}=80,71(78,05 ; 83,34)$ \\
\hline$\lambda_{01}=50 \%$ & Negativo & 208 & 327 & 0 & $74,03<S_{P}<84,92$ & $\widehat{S_{P}}=84,17(80,02 ; 88,25)$ \\
\hline
\end{tabular}

Nota-se que, quanto maior a proporção de indivíduos não verificados, maiores são os limites para sensibilidade e especificidade (casos $(b)$ e $(c)$ ).

Agora, considerando-se os dados do Exemplo 2 (Higgins e Keller, 1973), foram supostas as mesmas situações do exemplo anterior para indivíduos não verificados. Utilizandose os métodos de Begg e Greenes (1983) e Harel e Zhou (2006), algumas simulações foram feitas para estimação da sensibilidade, especificidade e seus intervalos de confiança. Os resultados obtidos são dados na Tabela 16 (dados completos - painel $(a)$ ). Nota-se que, nos painéis $(b)$ e $(c)$, os valores da sensibilidade e especificidade são praticamente iguais aos mesmos, quando todos os indivíduos são verificados $\left(\widehat{S_{E}}=20,25 \%\right.$ e $\widehat{S_{P}}=$ 91,32\%). Isso ocorre, pois o método proposto por Begg e Greenes é eficiente quando se tem $k_{1}=k_{2}=1$, ou seja, $k_{1}=\frac{\lambda_{11}}{\lambda_{01}}$ e $k_{2}=\frac{\lambda_{10}}{\lambda_{00}}$. Verifica-se, também, que à medida que a proporção de indivíduos não verificados aumenta, os erros padrão das estimativas sofrem um suave aumento. Já nas situações $(d),(e),(f)$ e $(g)$ as estimativas começam a sofrer alterações, dependendo da proporção retirada dos indivíduos verificados. Esses resultados são esperados, pois, agora, não se tem mais que $k_{1}=k_{2}=1$, e o método de Begg e Greenes (1983) mostra-se limitado para a estimação de sensibilidade e especifidade. Utilizando-se a metodologia proposta por Zhou (1993), as mesmas situações foram consideradas, e os resultados encontrados são dados na Tabela 17. 
Tabela 16: Estimativas de sensibilidade e especificidade - Método de Begg e Greenes.

\begin{tabular}{|c|c|c|c|c|c|c|}
\hline Painel & $\begin{array}{c}\text { Resultado } \\
\text { do Teste }\end{array}$ & \multicolumn{2}{|c|}{ Verificados pelo P. O. } & $\begin{array}{c}\text { Não } \\
\text { Verificados }\end{array}$ & $\begin{array}{r}\text { Estimativas } \\
\text { de B\&G (\%) } \\
\end{array}$ & $\begin{array}{c}\text { Estimativas } \\
\text { Logit B\&G }(\%) \\
\end{array}$ \\
\hline (a) $\lambda_{11}=\lambda_{00}=100 \%$ & Positivo & 65 & 134 & - & $\widehat{S_{E}}=20,25(10,48 ; 30,02)$ & $\widehat{S_{E}}=20,25(16,21 ; 25,00)$ \\
\hline$\lambda_{01}=\lambda_{10}=100 \%$ & Negativo & 256 & 1409 & - & $\widehat{S_{P}}=91,32(89,85 ; 92,79)$ & $\widehat{S_{P}}=91,32(89,80 ; 92,62)$ \\
\hline (b) $\lambda_{11}=\lambda_{00}=80 \%$ & Positivo & 52 & 107 & 30 & $\widehat{S_{E}}=20,21(9,72 ; 30,94)$ & $\widehat{S_{E}}=20,21(15,96 ; 25,42)$ \\
\hline$\lambda_{01}=\lambda_{10}=80 \%$ & Negativo & 205 & 1127 & 333 & $\widehat{S_{P}}=91,33(89,84 ; 92,86)$ & $\widehat{S_{P}}=91,33(89,72 ; 92,74)$ \\
\hline (c) $\lambda_{11}=\lambda_{00}=50 \%$ & Positivo & 33 & 67 & 99 & $\widehat{S_{E}}=20,43(7,72 ; 33,11)$ & $\widehat{S_{E}}=20,43(15,34 ; 26,86)$ \\
\hline$\lambda_{01}=\lambda_{10}=50 \%$ & Negativo & 128 & 705 & 832 & $\widehat{S_{P}}=91,47(89,71 ; 93,02)$ & $\widehat{S_{P}}=91,47(89,63 ; 92,81)$ \\
\hline (d) $\lambda_{11}=50 \%$ & Positivo & 33 & 134 & 32 & $\widehat{S_{E}}=13,34(2,01 ; 24,62)$ & $\widehat{S_{E}}=13,34(9,72 ; 18,03)$ \\
\hline$\lambda_{00}=\lambda_{01}=\lambda_{10}=100 \%$ & Negativo & 256 & 1409 & 0 & $\widehat{S_{P}}=89,84(88,25 ; 91,46)$ & $\widehat{S_{P}}=89,84(88,21 ; 91,23)$ \\
\hline (e) $\lambda_{11}=\lambda_{00}=50 \%$ & Positivo & 33 & 134 & 32 & $\widehat{S_{E}}=8,14(0,11 ; 17,43)$ & $\widehat{S_{E}}=8,14(5,96 ; 11,27)$ \\
\hline$\lambda_{01}=\lambda_{10}=100 \%$ & Negativo & 256 & 705 & 704 & $\widehat{S_{P}}=88,43(86,64 ; 90,35)$ & $\widehat{S_{P}}=88,43(86,63 ; 90,14)$ \\
\hline (f) $\lambda_{11}=\lambda_{00}=\lambda_{01}=100 \%$ & Positivo & 65 & 134 & 0 & $\widehat{S_{E}}=31,94(20,45 ; 43,46)$ & $\widehat{S_{E}}=31,94(25,84 ; 38,71)$ \\
\hline$\lambda_{10}=50 \%$ & Negativo & 128 & 1409 & 128 & $\widetilde{S_{P}}=91,97(90,63 ; 93,34)$ & $\widehat{S_{P}}=91,97(90,54 ; 93,14)$ \\
\hline (g) $\lambda_{11}=\lambda_{00}=\lambda_{10}=100 \%$ & Positivo & 65 & 67 & 67 & $\widehat{S_{E}}=27,74(18,02 ; 37,35)$ & $\widehat{S_{E}}=27,74(22,94 ; 33,07)$ \\
\hline $\begin{aligned} & \lambda_{01}=50 \% \\
&\end{aligned}$ & Negativo & 256 & 1409 & 0 & $\widehat{S_{P}}=93,37(91,82 ; 94,87)$ & $\widehat{S_{P}}=93,37(91,83 ; 94,62)$ \\
\hline
\end{tabular}

Tabela 17: Limites para sensibilidade e especificidade - Método de Zhou.

\begin{tabular}{|c|c|c|c|c|c|c|}
\hline Painel & $\begin{array}{c}\text { Resultado } \\
\text { do Teste }\end{array}$ & $\begin{array}{l}\text { Verificados } \\
\text { Positivo } \\
\end{array}$ & $\begin{array}{l}\text { pelo P. O. } \\
\text { Negativo }\end{array}$ & $\begin{array}{c}\text { Não } \\
\text { Verificados }\end{array}$ & $\begin{array}{c}\text { Limites } \\
\text { Método de Zhou (\%) }\end{array}$ & $\begin{array}{l}\text { Estimativas } \\
\text { de B\&G }(\%)\end{array}$ \\
\hline (a) $\lambda_{11}=\lambda_{00}=100 \%$ & Positivo & 65 & 134 & - & - & $\widehat{S_{E}}=20,25(10,48 ; 30,02)$ \\
\hline$\lambda_{01}=\lambda_{10}=100 \%$ & Negativo & 256 & 1409 & - & - & $\widehat{S_{P}}=91,32(89,85 ; 92,79)$ \\
\hline (b) $\lambda_{11}=\lambda_{00}=80 \%$ & Positivo & 52 & 107 & 30 & $8,84<S_{E}<31,06$ & $\widehat{S_{E}}=20,21(9,72 ; 30,94)$ \\
\hline$\lambda_{01}=\lambda_{10}=80 \%$ & Negativo & 205 & 1127 & 333 & $88,57<S_{P}<93,26$ & $\widehat{S_{P}}=91,33(89,84 ; 92,86)$ \\
\hline (c) $\lambda_{11}=\lambda_{00}=50 \%$ & Positivo & 33 & 67 & 99 & $3,31<S_{E}<50,84$ & $\widehat{S_{E}}=20,43(7,72 ; 33,11)$ \\
\hline$\lambda_{01}=\lambda_{10}=50 \%$ & Negativo & 128 & 705 & 832 & $80,94<S_{P}<95,87$ & $\widehat{S_{P}}=91,47(89,71 ; 93,02)$ \\
\hline (d) $\lambda_{11}=50 \%$ & Positivo & 33 & 134 & 32 & $11,49<S_{E}<20,23$ & $\widehat{S_{E}}=13,34(2,01 ; 24,62)$ \\
\hline$\lambda_{00}=\lambda_{01}=\lambda_{10}=100 \%$ & Negativo & 256 & 1409 & 0 & $89,46<S_{P}<91,34$ & $\widehat{S_{P}}=89,84(88,25 ; 91,46)$ \\
\hline (e) $\lambda_{11}=\lambda_{00}=50 \%$ & Positivo & 33 & 134 & 32 & $3,32<S_{E}<20,24$ & $\widehat{S_{E}}=8,14(0,11 ; 17,43)$ \\
\hline$\lambda_{01}=\lambda_{10}=100 \%$ & Negativo & 256 & 705 & 704 & $80,94<S_{P}<91,35$ & $\widehat{S_{P}}=88,43(86,64 ; 90,35)$ \\
\hline \multirow{2}{*}{ (f) $\begin{array}{c}\lambda_{11}= \\
\lambda_{00}=\lambda_{01}=100 \% \\
\lambda_{10}=50 \%\end{array}$} & Positivo & 65 & 134 & 0 & $20,23<S_{E}<33,72$ & $\widehat{S_{E}}=31,94(20,45 ; 43,46)$ \\
\hline & Negativo & 128 & 1409 & 128 & $91,34<S_{P}<92,03$ & $\widehat{S_{P}}=91,97(90,63 ; 93,34)$ \\
\hline \multirow{2}{*}{$\begin{array}{c}\text { (g) } \lambda_{11}=\lambda_{00}=\lambda_{10}=100 \% \\
\lambda_{01}=50 \%\end{array}$} & Positivo & 65 & 67 & 67 & $20,21<S_{E}<34,06$ & $\widehat{S_{E}}=27,74(18,02 ; 37,35)$ \\
\hline & Negativo & 256 & 1409 & 0 & $91,35<S_{P}<95,51$ & $\widehat{S_{P}}=93,37(91,82 ; 94,87)$ \\
\hline
\end{tabular}

Considerando-se os dados do Exemplo 4 (MASUR e MONTEIRO, 1983) e utilizandose os métodos de Begg e Greenes (1983) e Harel e Zhou (2006), algumas simulações foram feitas para estimação da sensibilidade, especificidade e seus intervalos de confiança. As mesmas suposições de indivíduos não verificados, dadas nos dois exemplos anteriores, foram utilizadas. Os resultados obtidos estão na Tabela 18. 
Tabela 18: Estimativas de sensibilidade e especificidade - Método de Begg e Greenes.

\begin{tabular}{|c|c|c|c|c|c|c|}
\hline Painel & $\begin{array}{l}\text { Resultado } \\
\text { do Teste }\end{array}$ & \multicolumn{2}{|c|}{ Verificados pelo P. O. } & $\begin{array}{c}\text { Não } \\
\text { Verificados }\end{array}$ & $\begin{array}{l}\text { Estimativas } \\
\text { de B\&G }(\%)\end{array}$ & $\begin{array}{c}\text { Estimativas } \\
\text { Logit B\&G (\%) }\end{array}$ \\
\hline (a) $\lambda_{11}=\lambda_{00}=100 \%$ & Positivo & 60 & 8 & - & $\widehat{S_{E}}=88,24(80,08 ; 96,39)$ & $\widehat{S_{E}}=88,24(78,20 ; 94,01)$ \\
\hline$\lambda_{01}=\lambda_{10}=100 \%$ & Negativo & 8 & 38 & - & $\widehat{S_{P}}=82,61(70,56 ; 94,66)$ & $\widehat{S_{P}}=82,61(68,91 ; 91,06)$ \\
\hline (b) $\lambda_{11}=\lambda_{00}=80 \%$ & Positivo & 48 & 6 & 14 & $\widehat{S_{E}}=87,31(78,12 ; 96,54)$ & $\widehat{S_{E}}=87,31(76,03 ; 93,75)$ \\
\hline$\lambda_{01}=\lambda_{10}=80 \%$ & Negativo & 6 & 30 & 10 & $\widehat{S_{P}}=81,12(67,32 ; 94,87)$ & $\widehat{S_{P}}=81,12(65,74 ; 90,58)$ \\
\hline (c) $\lambda_{11}=\lambda_{00}=50 \%$ & Positivo & 30 & 4 & 34 & $\widehat{S_{E}}=88,25(77,56 ; 99,01)$ & $\widehat{S_{E}}=88,25(73,94 ; 95,27)$ \\
\hline$\lambda_{01}=\lambda_{10}=50 \%$ & Negativo & 4 & 19 & 23 & $\widehat{S_{P}}=82,67(66,64 ; 98,67)$ & $\widehat{S_{P}}=82,67(63,38 ; 92,98)$ \\
\hline (d) $\lambda_{11}=50 \%$ & Positivo & 30 & 8 & 30 & $\widehat{S_{E}}=87,04(77,94 ; 96,12)$ & $\widehat{S_{E}}=87,04(76,07 ; 93,47)$ \\
\hline$\lambda_{00}=\lambda_{01}=\lambda_{10}=100 \%$ & Negativo & 8 & 38 & 0 & $\widehat{S_{P}}=72,67(55,61 ; 89,73)$ & $\widehat{S_{P}}=72,67(56,17 ; 84,72)$ \\
\hline (e) $\lambda_{11}=\lambda_{00}=50 \%$ & Positivo & 30 & 8 & 30 & $\widehat{S_{E}}=79,84(66,97 ; 92,68)$ & $\widehat{S_{E}}=79,84(68,96 ; 88,94)$ \\
\hline$\lambda_{01}=\lambda_{10}=100 \%$ & Negativo & 8 & 19 & 19 & $\widehat{S_{P}}=69,37(49,97 ; 88,83)$ & $\widehat{S_{P}}=69,37(51,47 ; 82,97)$ \\
\hline (f) $\lambda_{11}=\lambda_{00}=\lambda_{01}=100 \%$ & Positivo & 60 & 8 & 0 & $\widehat{S_{E}}=93,28(86,62 ; 99,87)$ & $\widehat{S_{E}}=93,28(83,32 ; 97,49)$ \\
\hline$\lambda_{10}=50 \%$ & Negativo & 4 & 38 & 4 & $\widehat{S_{P}}=83,94(72,76 ; 95,14)$ & $\widehat{S_{P}}=83,94(70,94 ; 91,73)$ \\
\hline (g) $\lambda_{11}=\lambda_{00}=\lambda_{10}=100 \%$ & Positivo & 60 & 4 & 4 & $\widehat{S_{E}}=88,91(81,17 ; 96,69)$ & $\widehat{S_{E}}=88,91(79,37 ; 94,36)$ \\
\hline$\lambda_{01}=50 \%$ & Negativo & 8 & 38 & 0 & $\widehat{S_{P}}=89,92(80,12 ; 99,87)$ & $\widehat{S_{P}}=89,92(76,23 ; 96,27)$ \\
\hline
\end{tabular}

Aqui, também, se nota que, nos painéis $(b)$ e $(c)$, os valores da sensibilidade e especificidade são praticamente iguais aos obtidos, quando todos os indivíduos são verificados $\left(\widehat{S_{E}}=88,21 \%\right.$ e $\left.\widehat{S_{P}}=82,63 \%\right)$. Isso ocorre, pois o método proposto por Begg e Greenes é eficiente quando se tem $k_{1}=k_{2}=1$, ou seja, $k_{1}=\frac{\lambda_{11}}{\lambda_{01}}$ e $k_{2}=\frac{\lambda_{10}}{\lambda_{00}}$. Verifica-se que à medida que a proporção de indivíduos não verificados aumenta, os erros padrão das estimativas sofrem um suave aumento. Já nas situações $(d),(e),(f)$ e $(g)$, as estimativas começam a sofrer alterações, dependendo da proporção retirada dos indivíduos verificados. Esses resultados são esperados, pois, agora, não se tem mais que $k_{1}=k_{2}=1$, e o método de Begg e Greenes (1983) mostra-se limitado para a estimação de sensibilidade e especifidade. Utilizando-se a metodologia proposta por Zhou (1993), as mesmas situações foram consideradas, e os resultados encontrados estão na Tabela 19.

Os estimadores de $S_{E}$ e $S_{P}$ propostos por Begg e Greenes (1983) são largamente utilizados por pesquisadores da área médica, entretanto essa metodologia assume que a seleção do paciente para verificação depende apenas do resultado do teste e não do estado real da doença, o que pode não ser um fato que reflete a realidade. Já os estimadores de máxima verossimilhança propostos por Zhou (1993) são uma alternativa para os introduzidos por Begg e Greenes, mas eles são baseados na escolha de $k_{1}=\frac{\lambda_{11}}{\lambda_{01}}$ e $k_{2}=\frac{\lambda_{10}}{\lambda_{00}}$, que, quando escolhidos de maneira equivocada, podem resultar em estimativas ruins para $S_{E}$ e $S_{P}$. Essa é uma grande limitação do método, além de ele apenas fornecer limites e não estimativas pontuais. 
Tabela 19: Limites para sensibilidade e especificidade - Método de Zhou.

\begin{tabular}{|c|c|c|c|c|c|c|}
\hline \multirow[t]{2}{*}{ Painel } & \multirow{2}{*}{$\begin{array}{c}\text { Resultado } \\
\text { do Teste }\end{array}$} & \multicolumn{2}{|c|}{ Verificados pelo P. O. } & \multirow{2}{*}{$\begin{array}{c}\text { Não } \\
\text { Verificados }\end{array}$} & \multirow{2}{*}{$\begin{array}{c}\text { Limites } \\
\text { Método de Zhou (\%) }\end{array}$} & \multirow{2}{*}{$\begin{array}{l}\text { Estimativas } \\
\text { de B\&G }(\%)\end{array}$} \\
\hline & & Positivo & Negativo & & & \\
\hline (a) $\lambda_{11}=\lambda_{00}=100 \%$ & Positivo & 60 & 8 & - & - & $\widehat{S_{E}}=88,24(80,08 ; 96,39)$ \\
\hline$\lambda_{01}=\lambda_{10}=100 \%$ & Negativo & 8 & 38 & - & - & $\widehat{S_{P}}=82,61(70,56 ; 94,66)$ \\
\hline (b) $\lambda_{11}=\lambda_{00}=80 \%$ & Positivo & 48 & 6 & 14 & $76,23<S_{E}<89,64$ & $\widehat{S_{E}}=87,31(78,12 ; 96,54)$ \\
\hline$\lambda_{01}=\lambda_{10}=80 \%$ & Negativo & 6 & 30 & 10 & $61,25<S_{P}<84,42$ & $\widehat{S_{P}}=81,12(67,32 ; 94,87)$ \\
\hline (c) $\lambda_{11}=\lambda_{00}=50 \%$ & Positivo & 30 & 4 & 34 & $52,67<S_{E}<94,12$ & $\widehat{S_{E}}=88,25(77,56 ; 99,01)$ \\
\hline$\lambda_{01}=\lambda_{10}=50 \%$ & Negativo & 4 & 19 & 23 & $33,32<S_{P}<91,36$ & $\widehat{S_{P}}=82,67(66,64 ; 98,67)$ \\
\hline (d) $\lambda_{11}=50 \%$ & Positivo & 30 & 8 & 30 & $78,96<S_{E}<88,25$ & $\widehat{S_{E}}=87,04(77,94 ; 96,12)$ \\
\hline$\lambda_{00}=\lambda_{01}=\lambda_{10}=100 \%$ & Negativo & 8 & 38 & 0 & $50,02<S_{P}<82,62$ & $\widehat{S_{P}}=72,67(55,61 ; 89,73)$ \\
\hline (e) $\lambda_{11}=\lambda_{00}=50 \%$ & Positivo & 30 & 8 & 30 & $52,67<S_{E}<88,25$ & $\widehat{S_{E}}=79,84(66,97 ; 92,68)$ \\
\hline$\lambda_{01}=\lambda_{10}=100 \%$ & Negativo & 8 & 19 & 19 & $33,32<S_{P}<82,62$ & $\widehat{S_{P}}=69,37(49,97 ; 88,83)$ \\
\hline \multirow{2}{*}{ (f) $\begin{array}{c}\lambda_{11}= \\
\lambda_{00}=\lambda_{01}=100 \% \\
\lambda_{10}=50 \%\end{array}$} & Positivo & 60 & 8 & 0 & $88,25<S_{E}<93,86$ & $\widehat{S_{E}}=93,28(86,62 ; 99,87)$ \\
\hline & Negativo & 4 & 38 & 4 & $82,67<S_{P}<84,06$ & $\widehat{S_{P}}=83,94(72,76 ; 95,14)$ \\
\hline \multirow{2}{*}{ 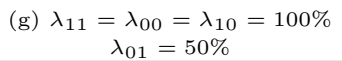 } & Positivo & 60 & 4 & 4 & $88,25<S_{E}<88,97$ & $\widehat{S_{E}}=88,91(81,17 ; 96,69)$ \\
\hline & Negativo & 8 & 38 & 0 & $82,67<S_{P}<90,51$ & $\widehat{S_{P}}=89,92(80,12 ; 99,87)$ \\
\hline
\end{tabular}

\subsection{Método Bayesiano}

Martinez et al. (2006) apresentaram uma metodologia sob o enfoque bayesiano para a estimação de $\lambda_{11}, \lambda_{10}, \lambda_{01}, \lambda_{00}, S_{E}, S_{P}$ e $\xi$. Assumindo-se independência a priori entre esses parâmetros, considerem-se as seguintes densidades a priori:

$$
\begin{aligned}
\lambda_{i j} & \sim \operatorname{Beta}\left(\alpha_{i j}, \beta_{i j}\right) \\
S_{E} & \sim \operatorname{Beta}\left(\alpha_{S_{E}}, \beta_{S_{E}}\right) \\
S_{P} & \sim \operatorname{Beta}\left(\alpha_{S_{P}}, \beta_{S_{P}}\right) \\
\xi & \sim \operatorname{Beta}\left(\alpha_{\xi}, \beta_{\xi}\right),
\end{aligned}
$$

em que $\alpha_{S_{E}}, \beta_{S_{E}}, \alpha_{S_{P}}, \beta_{S_{P}}, \alpha_{\xi}, \beta_{\xi}, \alpha_{i j}$ e $\beta_{i j}(i=0,1 ; j=0,1)$ são hiperparâmetros conhecidos, e $\operatorname{Beta}(a, b)$ denota uma distribuição Beta dada por $\pi(x) \propto x^{a-1}(1-x)^{b-1}$, $0<x<1$, com média $a /(a+b)$ e variância $(a b) /\left[(a+b)^{2}(a+b+1)\right]$. Para se obter melhor desempenho do algoritmo do amostrador de Gibbs, foram introduzidas variáveis latentes, dadas por $D_{i}^{*}=\left(D_{i 1}^{*}, D_{i 2}^{*}\right)($ ACHCAR et al., 2005):

(a) $D_{i 1}^{*}$, dado $V_{i}=0$ e $T_{i}=1$, é uma variável aleatória com distribuição de Bernoulli com probabilidade de sucesso dada por

$$
h_{1 i}=\frac{\xi\left(1-\lambda_{11}\right) S_{E}}{\xi\left(1-\lambda_{11}\right) S_{E}+\left(1-\lambda_{01}\right)\left(1-S_{P}\right)(1-\xi)} .
$$


Observe-se que $h_{1 i}=P\left(D_{i 1}^{*} \mid \bar{V}_{i} T_{i}\right)$. Portanto, a distribuição conjunta para $D_{i 1}^{*}, i=$ $1, \ldots, n$, é dada por

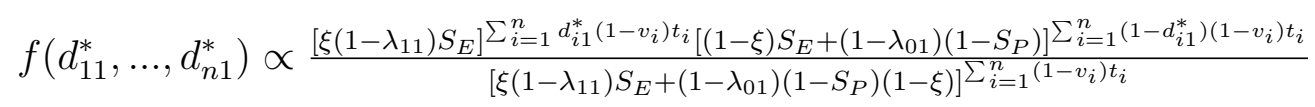

(b) $D_{i 2}^{*}$, dado $V_{i}=0$ e $T_{i}=1$, é uma variável aleatória com distribuição de Bernoulli com probabilidade de sucesso dada por

$$
h_{2 i}=\frac{\left(1-\lambda_{10}\right)\left(1-S_{E}\right) \xi}{\left(1-\lambda_{10}\right)\left(1-S_{E}\right) \xi+\left(1-\lambda_{00}\right)(1-\xi) S_{P}} .
$$

Observe-se que $h_{2 i}=P\left(D_{i 2}^{*} \mid \bar{V}_{i} \bar{T}_{i}\right)$. Portanto, a distribuição conjunta para $D_{i 2}^{*}, i=$ $1, \ldots, n$, é dada por

$$
f\left(d_{12}^{*}, \ldots, d_{n 2}^{*}\right) \propto \frac{\left[\left(1-\lambda_{10}\right)\left(1-S_{E}\right) \xi\right]^{\sum_{i=1}^{n} d_{i 2}^{*}\left(1-v_{i}\right)\left(1-t_{i}\right)}\left[\left(1-\lambda_{00}\right)(1-\xi) S_{P}\right]^{\sum_{i=1}^{n}\left(1-d_{i 2}^{*}\right)\left(1-v_{i}\right)\left(1-t_{i}\right)}}{\left[\left(1-\lambda_{10}\right)\left(1-S_{E}\right) \xi+\left(1-\lambda_{00}\right)(1-\xi) S_{P}\right]^{\sum_{i=1}^{n}\left(1-v_{i}\right)\left(1-t_{i}\right)}}
$$

Combinando-se (23), (33), (35) e (37), a distribuição a posteriori conjunta para $\boldsymbol{\theta}_{1}=$ $\left(\lambda_{11}, \lambda_{10}, \lambda_{01}, \lambda_{00}, S_{E}, S_{P}, \xi\right)^{\prime}$ é dada por

$$
\begin{aligned}
\pi\left(\boldsymbol{\theta}_{1}, d^{*} \mid \mathcal{D}\right)= & \xi^{\alpha_{\xi}+G_{1}-1}(1-\xi)^{\beta_{\xi}+G_{2}-1} S_{E}^{\alpha_{S_{E}}+G_{3}-1}\left(1-S_{E}\right)^{\beta_{S_{E}}+G_{4}-1} \\
& \times S_{P}^{\alpha_{S_{P}}+G_{5}-1}\left(1-S_{P}\right)^{\beta_{S_{P}}+G_{6}-1} \\
& \times \lambda_{01}^{\alpha_{21}+G_{7}-1}\left(1-\lambda_{01}\right)^{\beta_{21}+G_{8}-1} \lambda_{10}^{\alpha_{12}+G_{9}-1}\left(1-\lambda_{10}\right)^{\beta_{12}+G_{10}-1} \\
& \times \lambda_{00}^{\alpha_{22}+G_{11}-1}\left(1-\lambda_{00}\right)^{\beta_{22}+G_{12}-1} \lambda_{11}^{\alpha_{11}+G_{13}-1}\left(1-\lambda_{11}\right)^{\beta_{11}+G_{14}-1}
\end{aligned}
$$


em que

$$
\begin{aligned}
G_{1} & =\sum_{i=1}^{n} d_{i} v_{i}+\sum_{j=1}^{2} \sum_{i=1}^{n} d_{i j}^{*}\left(1-v_{i}\right), \\
G_{2} & =\sum_{i=1}^{n}\left(1-d_{i}\right)+\sum_{j=1}^{2} \sum_{i=1}^{n}\left(1-d_{i j}^{*}\right)\left(1-v_{i}\right), \\
G_{3} & =\sum_{i=1}^{n} d_{i} t_{i} v_{i}+\sum_{i=1}^{n} d_{i 1}^{*} t_{i}\left(1-v_{i}\right), \\
G_{4} & =\sum_{i=1}^{n} d_{i}\left(1-t_{i}\right) v_{i}+\sum_{i=1}^{n} d_{i 2}^{*}\left(1-t_{i}\right)\left(1-v_{i}\right), \\
G_{5} & =\sum_{i=1}^{n}\left(1-d_{i}\right)\left(1-t_{i}\right) v_{i}+\sum_{i=1}^{n} d_{i 2}^{*}\left(1-t_{i}\right)\left(1-v_{i}\right), \\
G_{6} & =\sum_{i=1}^{n}\left(1-d_{i}\right) t_{i} v_{i}+\sum_{i=1}^{n}\left(1-d_{i 1}^{*}\right) t_{i}\left(1-v_{i}\right), \\
G_{14} & =\sum_{i=1}^{n} d_{i 1}^{*} t_{i}\left(1-v_{i}\right) . \\
G_{13} & =\sum_{i=1}^{n}\left(1-d_{i}\right) t_{i} v_{i}, \\
G_{11} & =\sum_{i=1}^{n}\left(1-d_{i}\right)\left(1-t_{i}\right) v_{i}, \\
G_{10} & =\sum_{i=1}^{n}\left(1-d_{i 1}^{*}\right) t_{i}\left(1-v_{i}\right), \\
G_{9} & =\sum_{i=1}^{n} d_{i}\left(1-t_{i}\right) v_{i}, \\
G_{i 2}\left(1-t_{i}\right)\left(1-v_{i}\right), & \\
G_{i} & \\
G_{i} &
\end{aligned}
$$


As distribuições condicionais para o algoritmo do amostrador de Gibbs são dadas por

$$
\begin{aligned}
& \pi\left(\xi \mid \lambda_{11}, \lambda_{10}, \lambda_{01}, \lambda_{00}, S_{E}, S_{P}, \mathbf{d}^{*}, \mathcal{D}\right) \propto \xi^{\alpha_{\xi}+G_{1}-1}(1-\xi)^{\beta_{\xi}+G_{2}-1} \\
& \pi\left(S_{E} \mid \lambda_{11}, \lambda_{10}, \lambda_{01}, \lambda_{00}, S_{P}, \xi, \mathbf{d}^{*}, \mathcal{D}\right) \propto S_{E}^{\alpha_{S_{E}}+G_{3}-1}\left(1-S_{E}\right)^{\beta_{S_{E}}+G_{4}-1} \\
& \pi\left(S_{P} \mid \lambda_{11}, \lambda_{10}, \lambda_{01}, \lambda_{00}, S_{E}, \xi, \mathbf{d}^{*}, \mathcal{D}\right) \propto S_{P}^{\alpha_{S P}+G_{5}-1}\left(1-S_{P}\right)^{\beta_{S_{P}}+G_{6}-1} \\
& \pi\left(\lambda_{11} \mid \lambda_{10}, \lambda_{01}, \lambda_{00}, S_{E}, S_{P}, \xi, \mathbf{d}^{*}, \mathcal{D}\right) \propto \lambda_{11}^{\alpha_{11}+G_{13}-1}\left(1-\lambda_{11}\right)^{\beta_{11}+G_{14}-1} \\
& \pi\left(\lambda_{10} \mid \lambda_{11}, \lambda_{01}, \lambda_{00}, S_{E}, S_{P}, \xi, \mathbf{d}^{*}, \mathcal{D}\right) \propto \lambda_{10}^{\alpha_{12}+G_{9}-1}\left(1-\lambda_{10}\right)^{\beta_{12}+G_{10}-1} \\
& \pi\left(\lambda_{01} \mid \lambda_{11}, \lambda_{10}, \lambda_{00}, S_{E}, S_{P}, \xi, \mathbf{d}^{*}, \mathcal{D}\right) \propto \lambda_{01}^{\alpha_{21}+G_{7}-1}\left(1-\lambda_{01}\right)^{\beta_{21}+G_{8}-1} \\
& \pi\left(\lambda_{00} \mid \lambda_{11}, \lambda_{10}, \lambda_{01}, S_{E}, S_{P}, \xi, \mathbf{d}^{*}, \mathcal{D}\right) \propto \lambda_{00}^{\alpha_{22}+G_{11}-1}\left(1-\lambda_{00}\right)^{\beta_{22}+G_{12}-1}
\end{aligned}
$$

Portanto,

$$
\begin{aligned}
& \pi\left(\xi \mid \lambda_{11}, \lambda_{12}, \lambda_{21}, \lambda_{22}, S_{E}, S_{P}, \mathbf{d}^{*}, \mathcal{D}\right) \sim \operatorname{Beta}\left(\alpha_{\xi}+G_{1}, \beta_{\xi}+G_{2}\right) \\
& \pi\left(S_{E} \mid \lambda_{11}, \lambda_{12}, \lambda_{21}, \lambda_{22}, S_{P}, \xi, \mathbf{d}^{*}, \mathcal{D}\right) \sim \operatorname{Beta}\left(\alpha_{S_{E}}+G_{3}, \beta_{S_{E}}+G_{4}\right) \\
& \pi\left(S_{P} \mid \lambda_{11}, \lambda_{12}, \lambda_{21}, \lambda_{22}, S_{E}, \xi, \mathbf{d}^{*}, \mathcal{D}\right) \sim \operatorname{Beta}\left(\alpha_{S P}+G_{5}, \beta_{S_{P}}+G_{6}\right) \\
& \pi\left(\lambda_{11} \mid \lambda_{12}, \lambda_{21}, \lambda_{22}, S_{E}, S_{P}, \xi, \mathbf{d}^{*}, \mathcal{D}\right) \sim \operatorname{Beta}\left(\alpha_{11}+G_{13}, \beta_{11}+G_{14}\right) \\
& \pi\left(\lambda_{12} \mid \lambda_{11}, \lambda_{21}, \lambda_{22}, S_{E}, S_{P}, \xi, \mathbf{d}^{*}, \mathcal{D}\right) \sim \operatorname{Beta}\left(\alpha_{12}+G_{9}, \beta_{12}+G_{10}\right) \\
& \pi\left(\lambda_{21} \mid \lambda_{11}, \lambda_{12}, \lambda_{22}, S_{E}, S_{P}, \xi, \mathbf{d}^{*}, \mathcal{D}\right) \sim \operatorname{Beta}\left(\alpha_{21}+G_{7}, \beta_{21}+G_{8}\right) \\
& \pi\left(\lambda_{22} \mid \lambda_{11}, \lambda_{12}, \lambda_{21}, S_{E}, S_{P}, \xi, \mathbf{d}^{*}, \mathcal{D}\right) \sim \operatorname{Beta}\left(\alpha_{22}+G_{11}, \beta_{22}+G_{12}\right)
\end{aligned}
$$

Os passos para a geração das amostras da distribuição a posteriori conjunta, utilizandose o amostrador de Gibbs, são:

1) Propor valores iniciais para $\boldsymbol{\theta}_{1}^{(0)}=\left(\lambda_{11}^{(0)}, \lambda_{10}^{(0)}, \lambda_{01}^{(0)}, \lambda_{00}^{(0)}, S_{E}^{(0)}, S_{P}^{(0)}, \xi^{(0)}\right)$;

2) gerar uma amostra de $D_{k 1}^{*}=\left(D_{k 1}^{*}, \bar{D}_{k 1}^{*}\right), k=1, \ldots, n$, da distribuição de Bernoulli condicional, com probabilidade de sucesso (34) dado $T_{i}=1$ e $V_{i}=0$;

3) gerar uma amostra de $D_{k 2}^{*}=\left(D_{k 2}^{*}, \bar{D}_{k 2}^{*}\right), k=1, \ldots, n$, da distribuição de Bernoulli condicional, com probabilidade de sucesso (36) dado $T_{i}=0$ e $V_{i}=0$;

4) gerar amostras dos parâmetros de $\boldsymbol{\theta}_{1}$ a partir de suas respectivas distribuições 
condicionais;

5) voltar ao passo 2 até se obter convergência.

Considere-se a inclusão de um vetor de covariáveis $\mathbf{X}_{i}=\left(X_{0 i}, X_{1 i}, \ldots, X_{L i}\right)^{\prime}$, assumindose links logitos para $\xi, S_{E}, S_{P}, \lambda_{11}, \lambda_{01}, \lambda_{10}$ e $\lambda_{00}$ dados por

$$
v_{l i}=\exp \left(\sum_{j=0}^{L} \beta_{l j} x_{j i}\right)\left[1+\exp \left(\sum_{j=0}^{L} \beta_{l j} x_{j i}\right)\right]^{-1},
$$

para $l=1,2, \ldots, 7 ; X_{0 i}=1 ; v_{1 i}=\xi, v_{2 i}=S_{E}, v_{3 i}=S_{P}, v_{4 i}=\lambda_{11}, v_{5 i}=\lambda_{01}, v_{6 i}=\lambda_{10} \mathrm{e}$ $v_{7 i}=\lambda_{00}(i=1, \ldots, n)$. Assim, tem-se um vetor de parâmetros, dado por $\boldsymbol{\theta}_{2}=\left(\beta_{1}, \beta_{2}, \ldots\right.$, $\left.\beta_{7}\right)^{\prime}$, em que $\beta_{1}=\left(\beta_{10}, \beta_{11}, \ldots, \beta_{1 L}\right)^{\prime}, \beta_{2}=\left(\beta_{20}, \beta_{21}, \ldots, \beta_{2 L}\right)^{\prime}, \ldots, \beta_{7}=\left(\beta_{70}, \beta_{71}, \ldots, \beta_{7 L}\right)^{\prime}$. Considere-se independência a priori entre os parâmetros e densidades a priori para $\beta_{l j}$, dadas por:

$$
\beta_{l j} \sim N\left(a_{l j}, b_{l j}^{2}\right)
$$

com $a_{l j}$ e $b_{l j}$ conhecidos; $l=1,2, \ldots, 7$ e $j=0,1, \ldots, L$.

Combinando-se (35), (37), (40) e (23), a distribuição conjunta a posteriori para $\boldsymbol{\theta}_{2}=$ $\left(\beta_{1}, \beta_{2}, \ldots, \beta_{7}\right)^{\prime}$ e $\mathbf{d}^{*}$ é dada por

$$
\begin{aligned}
& \pi\left(\boldsymbol{\theta}_{2}, d^{*} \mid \mathcal{D}\right) \propto \pi\left(\boldsymbol{\theta}_{2}\right)\left[\prod_{i=1}^{n} \lambda_{00_{i}}^{r_{i}^{(001)}}\left(1-\lambda_{00_{i}}\right)^{s_{2 i}^{(000)}}\right]\left[\prod_{i=1}^{n} \lambda_{10_{i}}^{r_{i}^{(101)}}\left(1-\lambda_{10_{i}}\right)^{s_{2 i}^{(100)}}\right] \times \\
& \times\left[\prod_{i=1}^{n} \lambda_{0 i_{i}}^{r_{i}^{(011)}}\left(1-\lambda_{01_{i}}\right)^{s_{1 i}^{(010)}}\right]\left[\prod_{i=1}^{n} \lambda_{1 i_{i}}^{r_{i}^{(111)}}\left(1-\lambda_{11_{i}}\right)^{s_{1 i}^{(110)}}\right] \times \\
& \times\left[\prod_{i=1}^{n} S_{P_{i}}^{r_{i}^{(001)}+s_{2 i}^{(000)}}\left(1-S_{P_{i}}\right)^{r_{i}^{(011)}+s_{1 i}^{(010)}}\right] \times \\
& \times\left[\prod_{i=1}^{n} S_{E_{i}}^{r_{i}^{(111)}+s_{1 i}^{(110)}}\left(1-S_{E_{i}}\right)^{r_{i}^{(101)}+s_{2 i}^{(100)}}\right] \times
\end{aligned}
$$

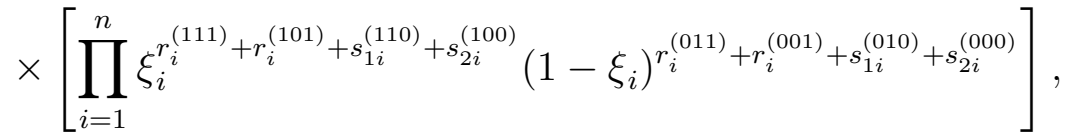

em que $\pi\left(\boldsymbol{\theta}_{2}\right)$ é a distribuição conjunta a priori para $\boldsymbol{\theta}_{2}, r_{i}^{(111)}=d_{i} t_{i} v_{i}, r_{i}^{(011)}=\left(1-d_{i}\right) t_{i} v_{i}$, $r_{i}^{(101)}=d_{i}\left(1-t_{i}\right) v_{i}, r_{i}^{(001)}=\left(1-d_{i}\right)\left(1-t_{i}\right) v_{i}, s_{1 i}^{(110)}=d_{i 1}^{*} t_{i}\left(1-v_{i}\right), s_{1 i}^{(010)}=\left(1-d_{i 2}^{*}\right) t_{i}\left(1-v_{i}\right)$, 
$s_{2 i}^{(100)}=d_{i 2}^{*}\left(1-t_{i}\right)\left(1-v_{i}\right), s_{2 i}^{(000)}=\left(1-d_{i 2}^{*}\right)\left(1-t_{i}\right)\left(1-v_{i}\right), i=1, \ldots, n$. Claramente, nota-se que as distribuições condicionais não possuem uma forma conhecida, então o algoritmo Metropolis - Hastings deve ser utilizado para simular as amostras a partir da distribuição conjunta a posteriori para $\boldsymbol{\theta}_{2}=\left(\beta_{1}, \beta_{2}, \ldots, \beta_{7}\right)^{\prime}$.

\subsubsection{Especificação das Distribuições a priori}

As informações a priori são representadas pela distribuição beta, cuja função de densidade é dada por

$$
f(\theta)=\frac{1}{B\left(\alpha_{\theta} ; \beta_{\theta}\right)} \theta^{\alpha_{\theta}-1}\left(1-\theta^{\beta_{\theta}-1}\right)
$$

se $0 \leq \theta \leq 1$, e $f(\theta)=0$, caso contrário; considerando $\alpha_{\theta}>0$ e $\beta_{\theta}>0$. A média e o desvio padrão de uma variável aleatória que possui distribuição beta, são, respectivamente, dadas por

$$
\frac{\alpha_{\theta}}{\alpha_{\theta}+\beta_{\theta}} \quad \text { e } \quad \sqrt{\frac{\alpha_{\theta} \beta_{\theta}}{\left(\alpha_{\theta}+\beta_{\theta}\right)^{2}\left(\alpha_{\theta}+\beta_{\theta}+1\right)}} \text {. }
$$

Suponha-se que, com base na literatura ou na opinião de especialistas, é conhecido que $\theta$ é um possível valor entre $\Lambda_{1}$ e $\Lambda_{2}$. Uma maneira de se especificar os hiperparâmetros da distribuição a priori é igualar o ponto médio entre $\Lambda_{1}$ e $\Lambda_{2}$ à média da distribuição beta. Por sua vez, o desvio padrão da distribuição beta pode ser assumido como equivalente a um quarto da amplitude do intervalo $\left(\Lambda_{1}, \Lambda_{2}\right)$ (ver BROWNE, 2001 e JOSEPH et al., 1995). Assim, os hiperparâmetros são especificados por

$$
\alpha_{\theta}=\frac{2\left[2\left(\Lambda_{1}+\Lambda_{2}\right)-\left(\Lambda_{1}+\Lambda_{2}\right)^{2}\right]}{\left(\Lambda_{2}+\Lambda_{1}\right)^{2}}-\frac{\left(\Lambda_{1}+\Lambda_{2}\right)}{2} \quad \text { e } \quad \beta_{\theta}=\frac{\alpha_{\theta}\left(2-\Lambda_{1}-\Lambda_{2}\right)}{\Lambda_{1}+\Lambda_{2}}
$$

Outra possibilidade, quando se dispõe, a priori, de uma estimativa "pontual", $m$, para $\theta$, além do intervalo $\left(\Lambda_{1}, \Lambda_{2}\right)$, é igualar a média da distribuição beta a $m$ e, ainda assumindo que o desvio padrão é equivalente a um quarto da amplitude $\Lambda_{1}-\Lambda_{2}$, os hiperparâmetros 
$\alpha_{\theta}$ e $\beta_{\theta}$ podem ser especificados como

$$
\alpha_{\theta}=\frac{16 m^{2}(1-m)}{\left(\Lambda_{2}+\Lambda_{1}\right)^{2}}-m \quad \text { e } \quad \beta_{\theta}=\frac{\alpha_{\theta}(1-m)}{m}
$$

\subsection{Exemplos}

Utilizando-se os dados dos exemplos ilustrados nas Tabelas 10 e 11 (DRUM e CHRISTACAPOULOS, 1972; SANTOS et al., 2003) foram realizadas algumas simulações, a partir da metodologia bayesiana. Para isso, foi escrito um programa na linguagem $R$, que é um software de livre acesso (http://www.r-project.org/). Considerando-se distribuições a priori não informativas (ver BOX e TIAO, 1992) e informativas, obtidas por meio dos estimadores de $S_{E}$ e $S_{P}$, introduzidos por Begg e Greenes (1983), foram geradas 90000 amostras, das quais as primeiras 5000 foram descartadas para que as inferências estivessem livres dos efeitos dos valores iniciais. Também foram consideradas apenas as $150^{a}, 300^{a}$, $450^{a}, \ldots$ iterações, resultando em 850 amostras de Gibbs. A convergência do algoritmo foi verificada a partir de gráficos temporais das amostras geradas e utilizando-se técnicas usuais existentes na literatura (GELMAN e RUBIN, 1992). Os resultados obtidos para os dados da Tabela 10 estão na Tabela 20.

Tabela 20: Sumários a posteriori e intervalos de credibilidade 95\% (ICr 95\%): (a) resultados obtidos a partir de distribuições a priori não informativas; (b) resultados obtidos a partir de distribuições a priori informativas para $S_{E}$ e $S_{P}$ ).

\begin{tabular}{|c|c|c|c|c|c|c|}
\hline & \multirow{3}{*}{ Parâmetro } & \multirow{3}{*}{$\begin{array}{c}\text { Distribuiçãa } \\
\text { a Priori }\end{array}$} & \multicolumn{2}{|c|}{ Sumários a Posteriori } & \multirow{2}{*}{\multicolumn{2}{|c|}{ ICr $95 \%$}} \\
\hline & & & Média & Desvio Padrão & & \\
\hline & & & & & LI & LS \\
\hline \multirow{7}{*}{$(a)$} & $\xi$ & $B(0.5,0.5)$ & 0,652 & 0,124 & 0,413 & $\overline{0,862}$ \\
\hline & $S_{E}$ & $B(0.5,0.5)$ & 0,788 & 0,104 & 0,596 & 0,937 \\
\hline & $S_{P}$ & $B(0.5,0.5)$ & 0,576 & 0,174 & 0,241 & 0,857 \\
\hline & $\lambda_{11}$ & $B(0.5,0.5)$ & 0,722 & 0,142 & 0,554 & 0,998 \\
\hline & $\lambda_{10}$ & $B(0.5,0.5)$ & 0,440 & 0,284 & 0,132 & 0,996 \\
\hline & $\lambda_{01}$ & $B(0.5,0.5)$ & 0,480 & 0,292 & 0,139 & 0,998 \\
\hline & $\lambda_{00}$ & $B(0.5,0.5)$ & 0,523 & 0,255 & 0,241 & 0,997 \\
\hline \multirow{7}{*}{ (b) } & $\xi$ & $B(0.5,0.5)$ & 0,500 & 0,067 & 0,401 & 0,654 \\
\hline & $S_{E}$ & $B(169.3,34.7)$ & 0,834 & 0,026 & 0,779 & 0,880 \\
\hline & $S_{P}$ & $B(73.3,25.8)$ & 0,544 & 0,024 & 0,497 & 0,591 \\
\hline & $\lambda_{11}$ & $B(0.5,0.5)$ & 0,846 & 0,082 & 0,692 & 0,996 \\
\hline & $\lambda_{10}$ & $B(0.5,0.5)$ & 0,644 & 0,195 & 0,312 & 0,994 \\
\hline & $\lambda_{01}$ & $B(0.5,0.5)$ & 0,190 & 0,052 & 0,113 & 0,319 \\
\hline & $\lambda_{00}$ & $B(0.5,0.5)$ & 0,319 & 0,053 & 0,234 & 0,442 \\
\hline
\end{tabular}

Os parâmetros $\alpha$ e $\beta$ da distribuição a priori informativa, beta, denotada por $B(\alpha, \beta)$, foram obtidos das estimativas de $S_{E}$ e $S_{P}$ e dos erros padrão, a partir do método de Begg 
e Greenes (1983). Assim, nesse caso, tem-se que uma distribuição beta com parâmetros 169,3 e 34,7 tem média igual a 0,836 e erro padrão igual a 0,027.

Nota-se que, quando utilizadas distribuições a priori informativas (painel (b)), os resultados de $S_{E}$ e $S_{P}$ ficam relativamente próximos aos obtidos pelo método de Begg e Greenes $\left(\widehat{S_{E}}=83,61 \% ; \widehat{S_{P}}=73,06 \%\right)$. Quando aplicado o método de Zhou (1993), encontram-se os limites $58,03 \%<S_{E}<93,63 \%$ para a sensibilidade e $21,47 \%<S_{P}<85,06 \%$ para a especificidade.

Os resultados obtidos para os dados da Tabela 11 estão na Tabela 21.

Tabela 21: Sumários a posteriori e intervalos de credibilidade 95\% (ICr 95\%): (a) resultados obtidos a partir de distribuições a priori não informativas; (b) resultados obtidos a partir de distribuições a priori informativas para $S_{E}$ e $S_{P}$ ).

\begin{tabular}{|c|c|c|c|c|c|c|}
\hline & \multirow{3}{*}{ Parâmetro } & \multirow{3}{*}{$\begin{array}{c}\text { Distribuição } \\
\text { a Priori }\end{array}$} & \multicolumn{2}{|c|}{ Sumários a Posteriori } & \multirow{2}{*}{\multicolumn{2}{|c|}{ ICr $95 \%$}} \\
\hline & & & Média & Desvio Padrão & & \\
\hline & & & & & LI & LS \\
\hline \multirow{7}{*}{ (a) } & $\xi$ & $B(0.5,0.5)$ & 0,255 & 0,059 & 0,151 & 0,377 \\
\hline & $S_{E}$ & $B(0.5,0.5)$ & 0,702 & 0,135 & 0,442 & 0,933 \\
\hline & $S_{P}$ & $B(0.5,0.5)$ & 0,500 & 0,055 & 0,392 & 0,607 \\
\hline & $\lambda_{11}$ & $B(0.5,0.5)$ & 0,907 & 0,084 & 0,705 & 0,999 \\
\hline & $\lambda_{10}$ & $B(0.5,0.5)$ & 0,489 & 0,280 & 0,095 & 0,997 \\
\hline & $\lambda_{01}$ & $B(0.5,0.5)$ & 0,947 & 0,046 & 0,938 & 0,999 \\
\hline & $\lambda_{00}$ & $B(0.5,0.5)$ & 0,855 & 0,099 & 0,664 & 0,999 \\
\hline \multirow{7}{*}{ (b) } & $\xi$ & $B(0.5,0.5)$ & 0,239 & 0,054 & 0,144 & 0,350 \\
\hline & $S_{E}$ & $B(7.2,1.6)$ & 0,772 & 0,103 & 0,551 & 0,935 \\
\hline & $S_{P}^{L}$ & $B(2.8,2.4)$ & 0,502 & 0,052 & 0,401 & 0,602 \\
\hline & $\lambda_{11}$ & $B(0.5,0.5)$ & 0,904 & 0,086 & 0,694 & 0,999 \\
\hline & $\lambda_{10}$ & $B(0.5,0.5)$ & 0,581 & 0,283 & 0,113 & 0,999 \\
\hline & $\lambda_{01}$ & $B(0.5,0.5)$ & 0,948 & 0,045 & 0,841 & 0,999 \\
\hline & $\lambda_{00}$ & $B(0.5,0.5)$ & 0,818 & 0,091 & 0,652 & 0,997 \\
\hline
\end{tabular}

Considerando-se que as estimativas de Begg e Greenes (1983) para sensibilidade e especificidade são dadas, respectivamente, por $\widehat{S_{E}}=82,31 \%$ e $\widehat{S_{P}}=57,15 \%$, pode-se verificar que os resultados obtidos pelo método bayesiano empírico (painel $(b)$ ) são muito próximos aos do método B\&G. Os limites de $S_{E}$ e $S_{P}$, propostos por Zhou, são dados, respectivamente por $54,87 \%<S_{E}<87,04 \%$ e $50,01 \%<S_{P}<59,04 \%$.

\subsection{Simulações}

Utilizando-se os dados completos dos Exemplos 1 (WEINER et al., 1979), 2 (HIGGINS e KELLER, 1973) e 7 (BEGG e MCNEIL, 1988) da Seção 1.4, foram realizadas simulações via método bayesiano, supondo-se diferentes porcentagens de indivíduos verificados pelo padrão ouro (P. O.), considerando-se distribuições a priori informativas e não informativas 
para sensibilidade e especificidade. Para cada conjunto de dados, inicialmente, foram retirados $10 \%$ de cada célula da tabela completa, depois 20\%, 30\% e 40\%, para se verificar como se comporta o método bayesiano na estimação da sensibilidade e da especificidade. Também foram consideradas distribuições a priori não informativas e informativas. Estas últimas foram encontradas por meio de simulações, quando retiradas amostras aleatórias de 10\%,20\%,30\% e 40\% do banco completo. Assim, quando retirada uma amostra de 20\%, a informação a priori foi utilizada para se estimarem os parâmetros encontrados a partir dos $80 \%$ restantes. Os resultados foram comparados aos obtidos pelo método de Begg e Greenes (1983).

\subsubsection{Diferentes Distribuições a priori}

Para os dados do Exemplo 1 (WEINER et al., 1979), foram feitas algumas simulações, considerando-se diferentes probabilidades $-\lambda_{11}, \lambda_{10}, \lambda_{01}$ e $\lambda_{00}-$ para os indivíduos verificados, mas sempre atendendo o pressuposto utilizado por Begg e Greenes (1983). Inicialmente, foram consideradas distribuições a priori não informativas. Já em um segundo momento, foram retiradas amostras aleatórias de 10\%, 20\%,30\% e 40\% do banco completo, a partir das quais foram calculadas distribuições a priori informativas e feitas novas simulações com os dados restantes. Para isso, foi escrito um programa na linguagem R. Foram geradas 90000 amostras, das quais as primeiras 5000 foram descartadas para que as inferências estivessem livres dos efeitos dos valores iniciais. Também foram consideradas apenas as $150^{a}, 300^{a}, 450^{a}, \ldots$ iterações, resultando em 850 amostras de Gibbs. A convergência do algoritmo foi verificada a partir de gráficos temporais das amostras geradas e utilizando-se técnicas usuais existentes na literatura (GELMAN e RUBIN, 1992) (ver Apêndices A e B). Os resultados encontram-se nas Tabelas 22, 23, 24, 25 e 26. 
Tabela 22: Resultados das simulações para cada porcentagem de verificação - dados de WEINER et al. (1979) - distribuições a priori não informativas.

\begin{tabular}{|c|c|c|c|c|c|c|c|}
\hline \multicolumn{2}{|r|}{ Painel } & $\begin{array}{c}\text { Resultado do } \\
\text { Teste }\end{array}$ & \multicolumn{2}{|c|}{ Verificados pelo P.O. } & $\begin{array}{c}\text { Não } \\
\text { Verificados }\end{array}$ & $\begin{array}{c}\text { Estimativas } \\
\text { de B\&G (\%) } \\
\end{array}$ & $\begin{array}{c}\text { Estimativas } \\
\text { Bayesianas (\%) }\end{array}$ \\
\hline \multirow[t]{2}{*}{ (a) } & $\lambda=100 \%$ & Positivo & 815 & 115 & - & $\widehat{S_{E}}=79,67(76,90 ; 82,43)$ & - \\
\hline & & Negativo & 208 & 327 & - & $\widehat{S_{P}}=73,98(69,23 ; 78,74)$ & - \\
\hline \multirow[t]{2}{*}{ (b) } & $\lambda=90 \%$ & Positivo & 734 & 104 & 92 & $\widehat{S_{E}}=79,66(76,82 ; 82,50)$ & $\widehat{S_{E}}=78,52(74,38 ; 82,42)$ \\
\hline & & Negativo & 187 & 294 & 54 & $\widehat{S_{P}}=73,91(68,96 ; 78,86)$ & $\widehat{S_{P}}=69,09(58,99 ; 78,02)$ \\
\hline \multirow[t]{2}{*}{ (c) } & $\lambda=80 \%$ & Positivo & 652 & 92 & 186 & $\widehat{S_{E}}=79,71(76,77 ; 82,64)$ & $\widehat{S_{E}}=77,26(70,56 ; 83,43)$ \\
\hline & & Negativo & 166 & 262 & 107 & $\widehat{S_{P}}=74,01(68,84 ; 79,19)$ & $\widehat{S_{P}}=64,78(49,28 ; 79,60)$ \\
\hline \multirow[t]{2}{*}{ (d) } & $\lambda=70 \%$ & Positivo & 571 & 81 & 278 & $\widehat{S_{E}}=79,63(76,58 ; 82,69)$ & $\widehat{S_{E}}=76,70(66,19 ; 85,39)$ \\
\hline & & Negativo & 146 & 229 & 160 & $\widehat{S_{P}}=73,87(68,40 ; 79,34)$ & $\widehat{S_{P}}=61,97(40,84 ; 82,02)$ \\
\hline \multirow[t]{2}{*}{ (e) } & $\lambda=60 \%$ & Positivo & 489 & 69 & 372 & $\widehat{S_{E}}=79,64(76,44 ; 82,84)$ & $\widehat{S_{E}}=75,20(60,19 ; 87,04)$ \\
\hline & & Negativo & 125 & 196 & 214 & $\widehat{S_{P}}=73,96(68,13 ; 78,79)$ & $\widehat{S_{P}}=59,73(32,30 ; 84,88)$ \\
\hline \multirow[t]{2}{*}{ (f) } & $\lambda=50 \%$ & Positivo & 408 & 58 & 464 & $\widehat{S_{E}}=79,65(76,25 ; 83,05)$ & $\widehat{S_{E}}=74,85(55,19 ; 89,19)$ \\
\hline & & Negativo & 104 & 164 & 268 & $\widehat{S_{P}}=73,92(67,64 ; 80,19)$ & $\widehat{S_{P}}=60,87(26,97 ; 87,68)$ \\
\hline \multirow[t]{2}{*}{ (g) } & $\lambda=40 \%$ & Positivo & 326 & 46 & 558 & $\widehat{S_{E}}=79,71(76,03 ; 83,38)$ & $\widehat{S_{E}}=73,38(48,85 ; 90,73)$ \\
\hline & & Negativo & 83 & 131 & 321 & $\widehat{S_{P}}=74,01(67,09 ; 80,93)$ & $\widehat{S_{P}}=55,92(21,12 ; 89,32)$ \\
\hline \multirow[t]{2}{*}{ (h) } & $\lambda=30 \%$ & Positivo & 245 & 35 & 650 & $\widehat{S_{E}}=79,70(75,59 ; 83,80)$ & $\widehat{S_{E}}=73,36(43,37 ; 93,20)$ \\
\hline & & Negativo & 62 & 98 & 375 & $\widehat{S_{P}}=73,81(65,94 ; 81,69)$ & $\widehat{S_{P}}=58,38(16,67 ; 92,24)$ \\
\hline \multirow{2}{*}{\multicolumn{2}{|c|}{ (i) $\lambda=20 \%$}} & Positivo & 163 & 23 & 744 & $\widehat{S_{E}}=79,51(74,69 ; 84,34)$ & $\widehat{S_{E}}=70,89(28,82 ; 95,30)$ \\
\hline & & Negativo & 42 & 65 & 428 & $\widehat{S_{P}}=73,86(64,31 ; 83,42)$ & $\widehat{S_{P}}=54,73(10,17 ; 94,92)$ \\
\hline \multirow{2}{*}{\multicolumn{2}{|c|}{ (j) $\lambda=10 \%$}} & Positivo & 82 & 12 & 836 & $\widehat{S_{E}}=79,59(73,05 ; 86,13)$ & $\widehat{S_{E}}=67,67(16,90 ; 97,53)$ \\
\hline & & Negativo & 21 & 33 & 481 & $\widehat{S_{P}}=73,36(60,14 ; 86,58)$ & $\widehat{S_{P}}=53,02(0,05 ; 97,30)$ \\
\hline
\end{tabular}

Tabela 23: Resultados das simulações para cada porcentagem de verificação - dados de WEINER et al. (1979) - disribuições a priori informativas (amostra aleatória de 10\%).

\begin{tabular}{|c|c|c|c|c|c|c|c|c|}
\hline \multicolumn{2}{|r|}{ Painel } & $\begin{array}{c}\text { Distribuição } \\
\text { a Priori }\end{array}$ & $\begin{array}{c}\text { Resultado } \\
\text { do Teste }\end{array}$ & \multicolumn{2}{|c|}{ Verificados pelo P.O. } & $\begin{array}{c}\text { Não } \\
\text { Verificados }\end{array}$ & $\begin{array}{c}\text { Estimativas } \\
\text { de B\&G }(\%)\end{array}$ & $\begin{array}{c}\text { Estimativas } \\
\text { Bayesianas (\%) }\end{array}$ \\
\hline & $\lambda=100 \%$ & - & Positivo & 815 & 115 & - & $\widehat{S_{E}}=79,67(76,90 ; 82,43)$ & - \\
\hline & & - & Negativo & 208 & 327 & - & $\widehat{S_{P}}=73,98(69,23 ; 78,74)$ & - \\
\hline \multirow[t]{2}{*}{ (b) } & $\lambda=90 \%$ & $S_{E} \sim B(24,6 ; 5,8)$ & Positivo & 659 & 96 & 77 & $\widehat{S_{E}}=78,94(75,89 ; 82,00)$ & $\widehat{S_{E}}=78,12(73,92 ; 82,00)$ \\
\hline & & $S_{P} \sim B(6,9 ; 3,4)$ & Negativo & 173 & 262 & 52 & $\widehat{S_{P}}=73,49(68,25 ; 78,74)$ & $\widehat{S_{P}}=68,91(59,91 ; 77,53)$ \\
\hline \multirow[t]{2}{*}{ (c) } & $\lambda=80 \%$ & $S_{E} \sim B(19,7 ; 5,2)$ & Positivo & 581 & 84 & 163 & $\widehat{S_{E}}=79,10(75,95 ; 82,24)$ & $\widehat{S_{E}}=77,56(71,03 ; 83,37)$ \\
\hline & & $S_{P} \sim B(4,4 ; 2,8)$ & Negativo & 153 & 240 & 98 & $\widehat{S_{P}}=74,14(68,75 ; 79,53)$ & $\widehat{S_{P}}=66,45(51,58 ; 79,47)$ \\
\hline \multirow[t]{2}{*}{ (d) } & $\lambda=70 \%$ & $S_{E} \sim B(9,6 ; 4,5)$ & Positivo & 525 & 65 & 255 & $\widehat{S_{E}}=80,08(76,93 ; 83,23)$ & $\widehat{S_{E}}=75,59(66,79 ; 84,50)$ \\
\hline & & $S_{P} \sim B(5,7 ; 4,1)$ & Negativo & 131 & 201 & 142 & $\widehat{S_{P}}=75,51(69,76 ; 81,25)$ & $\widehat{S_{P}}=60,83(40,62 ; 81,52)$ \\
\hline \multirow[t]{2}{*}{ (e) } & $\lambda=60 \%$ & $S_{E} \sim B(8,1 ; 4,2)$ & Positivo & 441 & 63 & 337 & $\widehat{S_{E}}=80,21(76,87 ; 83,55)$ & $\widehat{S_{E}}=74,13(62,54 ; 85,69)$ \\
\hline & & $S_{P} \sim B(2,9 ; 2,2)$ & Negativo & 109 & 178 & 191 & $\widehat{S_{P}}=73,82(67,70 ; 79,95)$ & $\widehat{S_{P}}=58,31(35,09 ; 82,32)$ \\
\hline \multirow[t]{2}{*}{ (f) } & $\lambda=50 \%$ & $S_{E} \sim B(6,8 ; 3,1)$ & Positivo & 362 & 56 & 425 & $\widehat{S_{E}}=79,38(75,80 ; 82,97)$ & $\widehat{S_{E}}=73,23(57,89 ; 86,93)$ \\
\hline & & $S_{P} \sim B(2,6 ; 1,9)$ & Negativo & 96 & 145 & 235 & $\widehat{S_{P}}=71,72(64,83 ; 78,60)$ & $\widehat{S_{P}}=56,22(28,71 ; 83,42)$ \\
\hline \multirow[t]{2}{*}{ (g) } & $\lambda=40 \%$ & $S_{E} \sim B(5,1 ; 2,4)$ & Positivo & 293 & 39 & 501 & $\widehat{S_{E}}=78,63(74,74 ; 82,51)$ & $\widehat{S_{E}}=71,67(52,74 ; 88,40)$ \\
\hline & & $S_{P} \sim B(2,5 ; 2,1)$ & Negativo & 81 & 116 & 289 & $\widehat{S_{P}}=74,52(67,12 ; 81,92)$ & $\widehat{S_{P}}=56,00(25,92 ; 85,86)$ \\
\hline \multirow[t]{2}{*}{ (h) } & $\lambda=30 \%$ & $S_{E} \sim B(2,9 ; 2,1)$ & Positivo & 220 & 31 & 583 & $\widehat{S_{E}}=80,08(75,04 ; 84,41)$ & $\widehat{S_{E}}=66,68(41,42 ; 88,42)$ \\
\hline & & $S_{P} \sim B(1,7 ; 1,6)$ & Negativo & 54 & 90 & 341 & $\widehat{S_{P}}=74,64(66,52 ; 82,75)$ & $\widehat{S_{P}}=51,88(16,21 ; 88,47)$ \\
\hline \multirow[t]{2}{*}{ (i) } & $\lambda=20 \%$ & $S_{E} \sim B(2,0 ; 1,7)$ & Positivo & 152 & 18 & 669 & $\widehat{S_{E}}=80,05(75,05 ; 85,06)$ & $\widehat{S_{E}}=67,56(34,13 ; 92,35)$ \\
\hline & & $S_{P} \sim B(1,9 ; 1,9)$ & Negativo & 37 & 58 & 385 & $\widehat{S_{P}}=76,74(66,98 ; 86,49)$ & $\widehat{S_{P}}=49,34(12,37 ; 87,91)$ \\
\hline \multirow[t]{2}{*}{ (j) } & $\lambda=10 \%$ & $S_{E} \sim B(2,0 ; 1,7)$ & Positivo & 72 & 10 & 748 & $\widehat{S_{E}}=78,68(71,91 ; 85,45)$ & $\widehat{S_{E}}=64,25(27,74 ; 93,20)$ \\
\hline & & $S_{P} \sim B(1,5 ; 1,5)$ & Negativo & 21 & 31 & 437 & $\widetilde{S_{P}}=74,23(60,19 ; 88,27)$ & $\overrightarrow{S_{P}}=46,24(9,35 ; 88,97)$ \\
\hline
\end{tabular}


Tabela 24: Resultados das simulações para cada porcentagem de verificação - dados de WEINER et al. (1979) - distribuições a priori informativas (amostra aleatória de 20\%).

\begin{tabular}{|c|c|c|c|c|c|c|c|c|}
\hline & Painel & $\begin{array}{l}\text { Distribuição } \\
\text { a Priori }\end{array}$ & $\begin{array}{l}\text { Resultado } \\
\text { do Teste }\end{array}$ & $\begin{array}{l}\text { Verificados } \\
\text { Positivo } \\
\end{array}$ & $\begin{array}{l}\text { pelo P.O. } \\
\text { Negativo }\end{array}$ & $\begin{array}{c}\text { Não } \\
\text { Verificados }\end{array}$ & $\begin{array}{c}\text { Estimativas } \\
\text { de B\&G }(\%) \\
\end{array}$ & $\begin{array}{c}\text { Estimativas } \\
\text { Bayesianas (\%) }\end{array}$ \\
\hline \multirow{2}{*}{\multicolumn{2}{|c|}{ (a) $\lambda=100 \%$}} & - & Positivo & 815 & 115 & - & $\widehat{S_{E}}=79,67(76,90 ; 82,43)$ & - \\
\hline & & - & Negativo & 208 & 327 & - & $\widehat{S_{P}}=73,98(69,23 ; 78,74)$ & - \\
\hline \multirow{2}{*}{\multicolumn{2}{|c|}{ (b) $\lambda=90 \%$}} & $S_{E} \sim B(37,1 ; 11,1)$ & Positivo & 580 & 85 & 76 & $\widehat{S_{E}}=79,31(76,10 ; 82,52)$ & $\widehat{S_{E}}=77,99(73,74 ; 81,96)$ \\
\hline & & $S_{P} \sim B(15,6 ; 5,4)$ & Negativo & 151 & 235 & 45 & $\widehat{S_{P}}=73,48(67,91 ; 79,04)$ & $\widehat{S_{P}}=68,00(58,93 ; 76,36)$ \\
\hline \multirow{2}{*}{\multicolumn{2}{|c|}{ (c) $\lambda=80 \%$}} & $S_{E} \sim B(28,0 ; 9,3)$ & Positivo & 518 & 72 & 148 & $\widehat{S_{E}}=79,86(76,58 ; 83,14)$ & $\widehat{S_{E}}=76,87(70,52 ; 82,86)$ \\
\hline & & $S_{P} \sim B(6,8 ; 4,5)$ & Negativo & 131 & 217 & 86 & $\widehat{S_{P}}=75,03(69,41 ; 80,65)$ & $\widehat{S_{P}}=65,25(50,56 ; 80,08)$ \\
\hline \multirow{2}{*}{\multicolumn{2}{|c|}{ (d) $\lambda=70 \%$}} & $S_{E} \sim B(16,6 ; 5,5)$ & Positivo & 462 & 66 & 226 & $\widehat{S_{E}}=79,48(76,08 ; 82,87)$ & $\widehat{S_{E}}=76,41(68,26 ; 84,19)$ \\
\hline & & $S_{P} \sim B(6,9 ; 4,0)$ & Negativo & 119 & 173 & 126 & $\widehat{S_{P}}=72,43(66,04 ; 78,83)$ & $\widehat{S_{P}}=62,15(42,74 ; 79,37)$ \\
\hline \multirow{2}{*}{\multicolumn{2}{|c|}{ (e) $\lambda=60 \%$}} & $S_{E} \sim B(11,6 ; 5,4)$ & Positivo & 393 & 53 & 306 & $\widehat{S_{E}}=80,66(77,19 ; 84,12)$ & $\widehat{S_{E}}=73,84(62,80 ; 84,39)$ \\
\hline & & $S_{P} \sim B(3,7 ; 2,8)$ & Negativo & 98 & 161 & 161 & $\widehat{S_{P}}=74,50(68,00 ; 81,00)$ & $\widehat{S_{P}}=55,29(32,71 ; 80,77)$ \\
\hline \multirow{2}{*}{\multicolumn{2}{|c|}{ (f) $\lambda=50 \%$}} & $S_{E} \sim B(8,0 ; 3,6)$ & Positivo & 321 & 48 & 371 & $\widehat{S_{E}}=79,15(75,29 ; 83,00)$ & $\widehat{S_{E}}=72,16(57,27 ; 86,47)$ \\
\hline & & $S_{P} \sim B(2,9 ; 2,3)$ & Negativo & 85 & 132 & 216 & $\widehat{S_{P}}=73,24(66,17 ; 80,30)$ & $\widehat{S_{P}}=57,53(29,37 ; 82,35)$ \\
\hline \multirow{2}{*}{\multicolumn{2}{|c|}{ (g) $\lambda=40 \%$}} & $S_{E} \sim B(5,9 ; 3,1)$ & Positivo & 263 & 37 & 436 & $\widehat{S_{E}}=79,78(75,72 ; 83,85)$ & $\widehat{S_{E}}=71,59(51,67 ; 87,84)$ \\
\hline & & $S_{P} \sim B(1,9 ; 1,8)$ & Negativo & 69 & 115 & 252 & $\widehat{S_{P}}=75,01(67,59 ; 82,43)$ & $\widehat{S_{P}}=52,36(25,55 ; 86,13)$ \\
\hline \multirow{2}{*}{\multicolumn{2}{|c|}{ (h) $\lambda=30 \%$}} & $S_{E} \sim B(4,4 ; 2,8)$ & Positivo & 200 & 23 & 518 & $\widehat{S_{E}}=80,60(76,14 ; 85,05)$ & $\widehat{S_{E}}=69,13(47,09 ; 88,16)$ \\
\hline & & $S_{P} \sim B(2,0 ; 2,1)$ & Negativo & 49 & 83 & 299 & $S_{P}=78,00(69,73 ; 86,26)$ & $S_{P}=55,08(20,94 ; 88,03)$ \\
\hline \multirow{2}{*}{\multicolumn{2}{|c|}{ (i) $\lambda=20 \%$}} & $S_{E} \sim B(2,6 ; 2,0)$ & Positivo & 132 & 23 & 604 & $\widehat{S_{E}}=79,73(74,26 ; 85,20)$ & $\widehat{S_{E}}=67,18(33,02 ; 92,51)$ \\
\hline & & $S_{P} \sim B(2,0 ; 1,7)$ & Negativo & 32 & 49 & 335 & $\widehat{S_{P}}=69,08(57,96 ; 80,21)$ & $\widehat{S_{P}}=44,56(10,94 ; 86,11)$ \\
\hline \multirow{2}{*}{\multicolumn{2}{|c|}{ (j) $\lambda=10 \%$}} & $S_{E} \sim B(2,3 ; 1,8)$ & Positivo & 61 & 12 & 664 & $\widehat{S_{E}}=76,91(68,96 ; 84,87)$ & $\widehat{S_{E}}=64,79(35,04 ; 87,03)$ \\
\hline & & $S_{P} \sim B(1,7 ; 1,6)$ & Negativo & 17 & 23 & 395 & $\widehat{S_{P}}=67,37(51,47 ; 83,27)$ & $\widehat{S_{P}}=50,75(10,08 ; 88,58)$ \\
\hline
\end{tabular}

Tabela 25: Resultados das simulações para cada porcentagem de verificação - dados de WEINER et al. (1979) - distribuições a priori informativas (amostra aleatória de 30\%).

\begin{tabular}{|c|c|c|c|c|c|c|c|c|}
\hline & Painel & $\begin{array}{l}\text { Distribuição } \\
\text { a Priori }\end{array}$ & $\begin{array}{l}\text { Resultado } \\
\text { do Teste }\end{array}$ & $\begin{array}{l}\text { Verificado } \\
\text { Positivo } \\
\end{array}$ & $\begin{array}{l}\text { pelo P.O. } \\
\text { Negativo }\end{array}$ & $\begin{array}{c}\text { Não } \\
\text { Verificados }\end{array}$ & $\begin{array}{c}\text { Estimativas } \\
\text { de B\&G }(\%) \\
\end{array}$ & $\begin{array}{c}\text { Estimativas } \\
\text { Bayesianas (\%) }\end{array}$ \\
\hline \multirow{2}{*}{\multicolumn{2}{|c|}{ (a) $\lambda=100 \%$}} & - & Positivo & 815 & 115 & - & $\widehat{S_{E}}=79,67(76,90 ; 82,43)$ & - \\
\hline & & - & Negativo & 208 & 327 & - & $\widehat{S_{P}}=73,98(69,23 ; 78,74)$ & - \\
\hline \multirow{2}{*}{\multicolumn{2}{|c|}{ (b) $\lambda=90 \%$}} & $S_{E} \sim B(51,6 ; 13,7)$ & Positivo & 504 & 71 & 67 & $\widehat{S_{E}}=79,09(75,64 ; 82,54)$ & $\widehat{S_{E}}=78,15(74,00 ; 82,03)$ \\
\hline & & $S_{P} \sim B(15,6 ; 8,3)$ & Negativo & 134 & 211 & 38 & $\widehat{S_{P}}=74,71(68,91 ; 80,52)$ & $\widehat{S_{P}}=69,76(60,07 ; 78,22)$ \\
\hline \multirow{2}{*}{\multicolumn{2}{|c|}{ (c) $\lambda=80 \%$}} & $S_{E} \sim B(37,1 ; 11,1)$ & Positivo & 449 & 66 & 126 & $\widehat{S_{E}}=79,62(76,06 ; 83,17)$ & $\widehat{S_{E}}=77,15(70,89 ; 83,28)$ \\
\hline & & $S_{P} \sim B(7,9 ; 5,3)$ & Negativo & 114 & 192 & 78 & $\widehat{S_{P}}=74,57(68,59 ; 80,56)$ & $\widehat{S_{P}}=64,83(50,69 ; 79,25)$ \\
\hline \multirow{2}{*}{\multicolumn{2}{|c|}{ (d) $\lambda=70 \%$}} & $S_{E} \sim B(27,1 ; 8,1)$ & Positivo & 392 & 55 & 190 & $\widehat{S_{E}}=78,72(74,99 ; 82,46)$ & $\widehat{S_{E}}=75,65(66,75 ; 83,63)$ \\
\hline & & $S_{P} \sim B(4,9 ; 3,7)$ & Negativo & 107 & 168 & 113 & $\widehat{S_{P}}=75,15(68,83 ; 81,47)$ & $\widehat{S_{P}}=62,84(44,29 ; 81,21)$ \\
\hline \multirow{2}{*}{\multicolumn{2}{|c|}{ (e) $\lambda=60 \%$}} & $S_{E} \sim B(13,8 ; 5,4)$ & Positivo & 337 & 52 & 254 & $\widehat{S_{E}}=80,15(76,27 ; 84,02)$ & $\widehat{S_{E}}=74,40(63,65 ; 85,97)$ \\
\hline & & $S_{P} \sim B(4,3 ; 3,2)$ & Negativo & 82 & 145 & 155 & $\widehat{S_{P}}=73,95(67,24 ; 80,66)$ & $\widehat{S_{P}}=60,46(36,62 ; 81,91)$ \\
\hline \multirow{2}{*}{\multicolumn{2}{|c|}{ (f) $\lambda=50 \%$}} & $S_{E} \sim B(9,5 ; 4,1)$ & Positivo & 277 & 39 & 337 & $\widehat{S_{E}}=79,67(75,64 ; 83,71)$ & $\widehat{S_{E}}=72,97(58,46 ; 85,57)$ \\
\hline & & $S_{P} \sim B(3,7 ; 2,9)$ & Negativo & 74 & 115 & 184 & $\widehat{S_{P}}=73,80(66,14 ; 81,45)$ & $\widehat{S_{P}}=56,09(30,79 ; 82,29)$ \\
\hline \multirow{2}{*}{\multicolumn{2}{|c|}{ (g) $\lambda=40 \%$}} & $S_{E} \sim B(6,9 ; 3,6)$ & Positivo & 226 & 30 & 403 & $\widehat{S_{E}}=79,84(75,53 ; 84,15)$ & $\widehat{S_{E}}=71,33(52,32 ; 87,28)$ \\
\hline & & $S_{P} \sim B(3,2 ; 2,7)$ & Negativo & 59 & 88 & 219 & $\widehat{S_{P}}=73,94(65,35 ; 82,53)$ & $\widehat{S_{P}}=49,77(24,12 ; 81,70)$ \\
\hline \multirow{2}{*}{\multicolumn{2}{|c|}{ (h) $\lambda=30 \%$}} & $S_{E} \sim B(3,8 ; 2,6)$ & Positivo & 177 & 22 & 467 & $\widehat{S_{E}}=81,35(76,72 ; 85,98)$ & $\widehat{S_{E}}=70,38(47,63 ; 88,31)$ \\
\hline & & $S_{P} \sim B(2,2 ; 2,0)$ & Negativo & 42 & 69 & 248 & $\widehat{S_{P}}=75,19(65,74 ; 84,64)$ & $\widehat{S_{P}}=54,69(18,71 ; 88,80)$ \\
\hline \multirow{2}{*}{\multicolumn{2}{|c|}{ (i) $\lambda=20 \%$}} & $S_{E} \sim B(3,0 ; 2,1)$ & Positivo & 115 & 18 & 522 & $\widehat{S_{E}}=81,00(75,37 ; 86,64)$ & $\widehat{S_{E}}=67,87(38,07 ; 90,28)$ \\
\hline & & $S_{P} \sim B(1,7 ; 1,6)$ & Negativo & 28 & 50 & 292 & $\widehat{S_{P}}=72,79(61,70 ; 83,89)$ & $\widehat{S_{P}}=51,24(17,38 ; 87,57)$ \\
\hline \multirow{2}{*}{\multicolumn{2}{|c|}{ (j) $\lambda=10 \%$}} & $S_{E} \sim B(2,3 ; 1,9)$ & Positivo & 54 & 9 & 596 & $\widehat{S_{E}}=80,05(72,39 ; 87,71)$ & $\widehat{S_{E}}=66,28(32,23 ; 92,69)$ \\
\hline & & $S_{P} \sim B(1,5 ; 1,5)$ & Negativo & 15 & 24 & 327 & $\widehat{S_{P}}=70,52(54,03 ; 87,01)$ & $\widehat{S_{P}}=49,09(9,08 ; 89,77)$ \\
\hline
\end{tabular}


Tabela 26: Resultados das simulações para cada porcentagem de verificação - dados de WEINER et al. (1979) - distribuições a priori informativas (amostra aleatória de 40\%).

\begin{tabular}{|c|c|c|c|c|c|c|c|}
\hline Painel & $\begin{array}{l}\text { Distribuição } \\
\text { a Priori } \\
\end{array}$ & $\begin{array}{l}\text { Resultado } \\
\text { do Teste }\end{array}$ & $\begin{array}{l}\text { Verificados } \\
\text { Positivo } \\
\end{array}$ & $\begin{array}{l}\text { pelo P.O. } \\
\text { Negativo } \\
\end{array}$ & $\begin{array}{c}\text { Não } \\
\text { Verificados } \\
\end{array}$ & $\begin{array}{c}\text { Estimativas } \\
\text { de B\&G }(\%) \\
\end{array}$ & $\begin{array}{c}\text { Estimativas } \\
\text { Bayesianas (\%) }\end{array}$ \\
\hline \multirow[t]{2}{*}{ (a) $\lambda=100 \%$} & - & Positivo & 815 & 115 & - & $\widehat{S_{E}}=79,67(76,90 ; 82,43)$ & - \\
\hline & - & Negativo & 208 & 327 & - & $\widehat{S_{P}}=73,98(69,23 ; 78,74)$ & - \\
\hline \multirow[t]{2}{*}{ (b) $\lambda=90 \%$} & $S_{E} \sim B(55,5 ; 18,5)$ & Positivo & 460 & 65 & 57 & $\widehat{S_{E}}=79,55(75,76 ; 83,37)$ & $\widehat{S_{E}}=77,99(74,11 ; 81,88)$ \\
\hline & $S_{P} \sim B(22,0 ; 8,5)$ & Negativo & 104 & 160 & 33 & $\widehat{S_{P}}=71,18(64,29 ; 78,09)$ & $\widehat{S_{P}}=68,17(58,64 ; 76,57)$ \\
\hline \multirow[t]{2}{*}{ (c) $\lambda=80 \%$} & $S_{E} \sim B(37,8 ; 11,9)$ & Positivo & 385 & 55 & 112 & $\widehat{S_{E}}=79,07(75,24 ; 82,90)$ & $\widehat{S_{E}}=76,32(70,28 ; 82,08)$ \\
\hline & $S_{P} \sim B(11,5 ; 6,8)$ & Negativo & 104 & 162 & 61 & $\widehat{S_{P}}=74,29(67,65 ; 80,89)$ & $\widehat{S_{P}}=64,86(51,64 ; 79,17)$ \\
\hline \multirow[t]{2}{*}{ (d) $\lambda=70 \%$} & $S_{E} \sim B(28,0 ; 9,3)$ & Positivo & 338 & 53 & 173 & $\widehat{S_{E}}=79,28(75,31 ; 83,25)$ & $\widehat{S_{E}}=76,35(68,12 ; 84,76)$ \\
\hline & $S_{P} \sim B(6,9 ; 4,0)$ & Negativo & 89 & 131 & 95 & $\widehat{S_{P}}=71,04(63,58 ; 78,51)$ & $\widehat{S_{P}}=62,88(43,13 ; 80,55)$ \\
\hline \multirow[t]{2}{*}{ (e) $\lambda=60 \%$} & $S_{E} \sim B(21,8 ; 8,1)$ & Positivo & 270 & 46 & 220 & $\widehat{S_{E}}=80,56(76,32 ; 84,81)$ & $\widehat{S_{E}}=73,32(60,85 ; 84,05)$ \\
\hline & $S_{P} \sim B(3,6 ; 3,1)$ & Negativo & 67 & 141 & 135 & $\widehat{S_{P}}=74,87(68,05 ; 81,70)$ & $\widehat{S_{P}}=57,61(38,01 ; 78,83)$ \\
\hline \multirow[t]{2}{*}{ (f) $\lambda=50 \%$} & $S_{E} \sim B(8,1 ; 4,4)$ & Positivo & 254 & 41 & 281 & $\widehat{S_{E}}=82,02(77,75 ; 86,29)$ & $\widehat{S_{E}}=72,75(57,98 ; 86,03)$ \\
\hline & $S_{P} \sim B(3,3 ; 2,5)$ & Negativo & 52 & 96 & 156 & $\widehat{S_{P}}=70,39(61,94 ; 78,83)$ & $\widehat{S_{P}}=51,34(25,44 ; 78,26)$ \\
\hline \multirow[t]{2}{*}{ (g) $\lambda=40 \%$} & $S_{E} \sim B(6,9 ; 3,6)$ & Positivo & 199 & 25 & 328 & $\widehat{S_{E}}=79,74(74,97 ; 84,51)$ & $\widehat{S_{E}}=70,07(53,11 ; 87,07)$ \\
\hline & $S_{P} \sim B(2,8 ; 2,4)$ & Negativo & 48 & 80 & 199 & $\widehat{S_{P}}=76,75(68,27 ; 85,23)$ & $\widehat{S_{P}}=54,00(26,23 ; 84,16)$ \\
\hline \multirow[t]{2}{*}{ (h) $\lambda=30 \%$} & $S_{E} \sim B(5,1 ; 2,8)$ & Positivo & 97 & 8 & 449 & $\widehat{S_{E}}=78,61(72,22 ; 84,99)$ & $\widehat{S_{E}}=68,49(48,26 ; 87,19)$ \\
\hline & $S_{P} \sim B(2,2 ; 1,9)$ & Negativo & 24 & 32 & 269 & $\widehat{S_{P}}=81,48(69,49 ; 93,46)$ & $\widehat{S_{P}}=58,12(20,14 ; 91,65)$ \\
\hline \multirow[t]{2}{*}{ (i) $\lambda=20 \%$} & $S_{E} \sim B(3,4 ; 2,3)$ & Positivo & 85 & 23 & 425 & $\widehat{S_{E}}=79,26(74,41 ; 84,12)$ & $\widehat{S_{E}}=66,74(41,00 ; 88,99)$ \\
\hline & $S_{P} \sim B(2,1 ; 2,0)$ & Negativo & 30 & 65 & 389 & $\widehat{S_{P}}=73,55(63,87 ; 83,23)$ & $\widehat{S_{P}}=46,78(16,07 ; 84,08)$ \\
\hline \multirow[t]{2}{*}{ (j) $\lambda=10 \%$} & $S_{E} \sim B(2,0 ; 1,7)$ & Positivo & 53 & 6 & 497 & $\widehat{S_{E}}=78,30(70,32 ; 86,27)$ & $\widehat{S_{E}}=65,20(24,84 ; 92,80)$ \\
\hline & $S_{P} \sim B(1,7 ; 1,7)$ & Negativo & 15 & 20 & 288 & $\widehat{S_{P}}=76,55(59,68 ; 93,42)$ & $\widehat{S_{P}}=47,47(10,72 ; 91,79)$ \\
\hline
\end{tabular}

Para os dados do Exemplo 2 (HIGGINS e KELLER, 1973), foram feitas algumas simulações considerando diferentes probabilidades $-\lambda_{11}, \lambda_{10}, \lambda_{01}$ e $\lambda_{00}-$ para os indivíduos verificados, mas sempre atendendo o pressuposto utilizado por Begg e Greenes (1983). A partir do banco completo, as proporções foram sendo diminuídas, para que se pudesse avaliar o quanto o método bayesiano é eficiente na estimação da sensibilidade e da especificidade dos testes diagnósticos. Inicialmente, foram consideradas distribuições a priori não informativas. Já em um segundo momento, foram retiradas amostras aleatórias de $10 \%, 20 \%, 30 \%$ e $40 \%$ do banco completo, a partir das quais foram calculadas distribuições a priori informativas e feitas novas simulações com os dados restantes. Os resultados encontram-se nas Tabelas 27, 28, 29, 30 e 31. 
Tabela 27: Resultados das simulações para cada porcentagem de verificação - dados de HIGGINS e KELLER (1973) - distribuições a priori não informativas.

\begin{tabular}{|c|c|c|c|c|c|c|c|}
\hline \multirow{2}{*}{\multicolumn{2}{|c|}{ Painel }} & \multirow{2}{*}{$\begin{array}{c}\text { Resultado } \\
\text { do Teste }\end{array}$} & \multicolumn{2}{|c|}{ Verificados pelo P.O. } & \multirow{2}{*}{$\begin{array}{c}\text { Não } \\
\text { Verificados } \\
\end{array}$} & \multirow{2}{*}{$\begin{array}{r}\text { Estimativas } \\
\text { de B\&G }(\%) \\
\end{array}$} & \multirow{2}{*}{$\begin{array}{c}\text { Estimativas } \\
\text { Bayesianas (\%) } \\
\end{array}$} \\
\hline & & & Positivo & Negativo & & & \\
\hline \multirow[t]{2}{*}{ (a) } & $\lambda=100 \%$ & Positivo & 65 & 134 & - & $\widehat{S_{E}}=20,25(10,48 ; 30,01)$ & - \\
\hline & & Negativo & 256 & 1409 & - & $\widehat{S_{P}}=91,32(89,85 ; 92,79)$ & - \\
\hline \multirow[t]{2}{*}{ (b) } & $\lambda=90 \%$ & Positivo & 58 & 121 & 30 & $\widehat{S_{E}}=20,94(10,74 ; 31,15)$ & $\widehat{S_{E}}=19,69(12,28 ; 29,16)$ \\
\hline & & Negativo & 230 & 1268 & 167 & $\widehat{S_{P}}=90,89(89,35 ; 92,43)$ & $\widehat{S_{P}}=90,83(88,68 ; 92,75)$ \\
\hline \multirow[t]{2}{*}{ (c) } & $\lambda=80 \%$ & Positivo & 52 & 107 & 40 & $\widehat{S_{E}}=20,25(0,10 ; 30,85)$ & $\widehat{S_{E}}=18,88(0,09 ; 31,25)$ \\
\hline & & Negativo & 205 & 1127 & 333 & $\widehat{S_{P}}=91,32(89,79 ; 92,85)$ & $\widehat{S_{P}}=91,17(88,23 ; 93,58)$ \\
\hline \multirow[t]{2}{*}{ (d) } & $\lambda=70 \%$ & Positivo & 46 & 94 & 59 & $\widehat{S_{E}}=20,36(0,09 ; 31,48)$ & $\widehat{S_{E}}=18,79(0,07 ; 36,53)$ \\
\hline & & Negativo & 179 & 986 & 500 & $\widehat{S_{P}}=91,34(89,78 ; 92,90)$ & $\widehat{S_{P}}=91,00(86,76 ; 94,26)$ \\
\hline \multirow[t]{2}{*}{ (e) } & $\lambda=60 \%$ & Positivo & 39 & 80 & 80 & $\widehat{S_{E}}=20,26(0,08 ; 32,11)$ & $\widehat{S_{E}}=19,16(0,06 ; 40,57)$ \\
\hline & & Negativo & 154 & 845 & 666 & $\widehat{S_{P}}=91,32(89,71 ; 92,94)$ & $\widehat{S_{P}}=90,24(83,91 ; 94,97)$ \\
\hline \multirow[t]{2}{*}{ (f) } & $\lambda=50 \%$ & Positivo & 33 & 67 & 99 & $\widehat{S_{E}}=20,43(0,08 ; 33,14)$ & $\widehat{S_{E}}=20,24(0,04 ; 47,79)$ \\
\hline & & Negativo & 128 & 705 & 832 & $\widehat{S_{P}}=91,36(89,68 ; 93,04)$ & $\overline{S_{P}}=91,45(81,62 ; 95,74)$ \\
\hline \multirow[t]{2}{*}{ (g) } & $\lambda=40 \%$ & Positivo & 26 & 54 & 119 & $\widehat{S_{E}}=20,23(0,06 ; 34,28)$ & $\widehat{S_{E}}=20,89(0,03 ; 57,35)$ \\
\hline & & Negativo & 102 & 564 & 999 & $\widehat{S_{P}}=91,30(89,52 ; 93,08)$ & $\widehat{S_{P}}=89,85(78,07 ; 96,65)$ \\
\hline \multirow[t]{2}{*}{ (h) } & $\lambda=30 \%$ & Positivo & 20 & 40 & 139 & $\widehat{S_{E}}=20,55(0,05 ; 36,33)$ & $\widehat{S_{E}}=21,56(0,04 ; 65,24)$ \\
\hline & & Negativo & 77 & 423 & 1165 & $\widehat{S_{P}}=91,39(89,46 ; 93,32)$ & $\widehat{S_{P}}=90,91(71,80 ; 97,24)$ \\
\hline \multirow[t]{2}{*}{ (i) } & $\lambda=20 \%$ & Positivo & 13 & 27 & 159 & $\widehat{S_{E}}=20,23(0,02 ; 39,38)$ & $\widehat{S_{E}}=20,34(0,03 ; 70,58)$ \\
\hline & & Negativo & 51 & 282 & 1332 & $\widehat{S_{P}}=91,30(89,10 ; 93,50)$ & $\widehat{S_{P}}=89,67(62,97 ; 98,48)$ \\
\hline \multirow{2}{*}{\multicolumn{2}{|c|}{ (j) $\lambda=10 \%$}} & Positivo & 7 & 13 & 179 & $\widehat{S_{E}}=21,18(0,01 ; 46,90)$ & $\widehat{S_{E}}=20,41(0,02 ; 76,05)$ \\
\hline & & Negativo & 26 & 141 & 1498 & $\widehat{S_{P}}=91,57(88,67 ; 94,47)$ & $\widehat{S_{P}}=88,57(52,29 ; 99,19)$ \\
\hline
\end{tabular}

Tabela 28: Resultados das simulações para cada porcentagem de verificação - dados de HIGGINS e KELLER (1973) - distribuições a priori informativas (amostra aleatória de $10 \%)$.

\begin{tabular}{|c|c|c|c|c|c|c|c|c|}
\hline & Painel & $\begin{array}{c}\text { Distribuição } \\
\text { a Priori }\end{array}$ & $\begin{array}{c}\text { Resultado } \\
\text { do Teste }\end{array}$ & $\begin{array}{l}\text { Verificados } \\
\text { Positivo }\end{array}$ & $\frac{\text { pelo P.O. }}{\text { Negativo }}$ & $\begin{array}{c}\text { Não } \\
\text { Verificados }\end{array}$ & $\begin{array}{c}\text { Estimativas } \\
\text { de B\&G (\%) }\end{array}$ & $\begin{array}{c}\text { Estimativas } \\
\text { Bayesianas (\%) }\end{array}$ \\
\hline \multirow{2}{*}{\multicolumn{2}{|c|}{ (a) $\lambda=100 \%$}} & - & Positivo & 65 & 134 & - & $\widehat{S_{E}}=20,25(10,48 ; 30,01)$ & - \\
\hline & & - & Negativo & 256 & 1409 & - & $\widehat{S_{P}}=91,32(89,85 ; 92,79)$ & - \\
\hline \multirow[t]{2}{*}{ (b) } & $\lambda=90 \%$ & $S_{E} \sim B(3,0 ; 12,0)$ & Positivo & 56 & 107 & 27 & $\widehat{S_{E}}=22,37(11,74 ; 33,01)$ & $\widehat{S_{E}}=20,17(12,21 ; 29,26)$ \\
\hline & & $S_{P} \sim B(60,6 ; 9,1)$ & Negativo & 203 & 1139 & 155 & $\widehat{S_{P}}=91,06(89,45 ; 92,67)$ & $\widehat{S_{P}}=90,73(88,47 ; 92,53)$ \\
\hline \multirow[t]{2}{*}{ (c) } & $\lambda=80 \%$ & $S_{E} \sim B(3,0 ; 9,0)$ & Positivo & 48 & 98 & 36 & $\widehat{S_{E}}=21,07(9,86 ; 32,28)$ & $\widehat{S_{E}}=17,37(9,77 ; 29,31)$ \\
\hline & & $S_{P} \sim B(34,0 ; 4,2)$ & Negativo & 178 & 1010 & 308 & $\widehat{S_{P}}=91,23(89,62 ; 92,85)$ & $\widehat{S_{P}}=90,48(87,90 ; 92,89)$ \\
\hline \multirow[t]{2}{*}{ (d) } & $\lambda=70 \%$ & $S_{E} \sim B(2,5 ; 4,5)$ & Positivo & 41 & 80 & 52 & $\widehat{S_{E}}=19,31(7,64 ; 30,98)$ & $\widehat{S_{E}}=17,60(6,41 ; 32,38)$ \\
\hline & & $S_{P} \sim B(30,5 ; 5,8)$ & Negativo & 161 & 889 & 455 & $\widehat{S_{P}}=92,20(90,64 ; 93,76)$ & $\widehat{S_{P}}=91,50(87,70 ; 94,35)$ \\
\hline \multirow[t]{2}{*}{ (e) } & $\lambda=60 \%$ & $S_{E} \sim B(2,6 ; 6,7)$ & Positivo & 36 & 72 & 75 & $\widehat{S_{E}}=21,13(8,64 ; 33,62)$ & $\widehat{S_{E}}=19,47(6,36 ; 39,46)$ \\
\hline & & $S_{P} \sim B(27,9 ; 4,5)$ & Negativo & 136 & 757 & 602 & $\widehat{S_{P}}=91,22(89,50 ; 92,94)$ & $\widehat{S_{P}}=90,54(85,48 ; 94,73)$ \\
\hline \multirow[t]{2}{*}{ (f) } & $\lambda=50 \%$ & $S_{E} \sim B(2,3 ; 3,9)$ & Positivo & 28 & 64 & 84 & $\widehat{S_{E}}=19,07(5,43 ; 32,71)$ & $\widehat{S_{E}}=22,59(5,52 ; 47,63)$ \\
\hline & & $S_{P} \sim B(21,3 ; 3,8)$ & Negativo & 112 & 628 & 762 & $\widehat{S_{P}}=91,23(89,49 ; 92,98)$ & $\widehat{S_{P}}=91,26(86,02 ; 95,38)$ \\
\hline \multirow[t]{2}{*}{ (g) } & $\lambda=40 \%$ & $S_{E} \sim B(1,7 ; 2,0)$ & Positivo & 24 & 50 & 101 & $\widehat{S_{E}}=19,27(4,78 ; 33,75)$ & $\widehat{S_{E}}=20,11(3,55 ; 50,00)$ \\
\hline & & $S_{P} \sim B(14,6 ; 6,4)$ & Negativo & 94 & 500 & 909 & $\widehat{S_{P}}=91,45(89,61 ; 93,29)$ & $\widehat{S_{P}}=90,35(80,45 ; 96,10)$ \\
\hline \multirow[t]{2}{*}{ (h) } & $\lambda=30 \%$ & $S_{E} \sim B(1,9 ; 3,0)$ & Positivo & 16 & 38 & 131 & $\widehat{S_{E}}=21,47(4,97 ; 37,97)$ & $\widehat{S_{E}}=24,09(4,96 ; 55,86)$ \\
\hline & & $S_{P} \sim B(17,5 ; 3,6)$ & Negativo & 68 & 374 & 1048 & $\widehat{S_{P}}=90,96(88,88 ; 93,04)$ & $\widehat{S_{P}}=89,94(80,57 ; 96,08)$ \\
\hline \multirow[t]{2}{*}{ (i) } & $\lambda=20 \%$ & $S_{E} \sim B(1,8 ; 2,3)$ & Positivo & 13 & 26 & 147 & $\widehat{S_{E}}=21,83(1,95 ; 41,71)$ & $\widehat{S_{E}}=23,71(4,03 ; 64,87)$ \\
\hline & & $S_{P} \sim B(10,5 ; 3,1)$ & Negativo & 43 & 246 & 1203 & $\widehat{S_{P}}=91,10(88,78 ; 93,43)$ & $\widehat{S_{P}}=88,37(72,26 ; 95,40)$ \\
\hline \multirow[t]{2}{*}{ (j) } & $\lambda=10 \%$ & $S_{E} \sim B(1,6 ; 1,7)$ & Positivo & 7 & 11 & 171 & $\widehat{S_{E}}=23,46(1,24 ; 47,32)$ & $\widehat{S_{E}}=26,83(2,60 ; 70,13)$ \\
\hline & & $S_{P} \sim B(5,1 ; 2,3)$ & Negativo & 24 & 125 & 1340 & $\widehat{S_{P}}=91,53(88,26 ; 94,81)$ & $\widehat{S_{P}}=87,00(59,98 ; 95,21)$ \\
\hline
\end{tabular}


Tabela 29: Resultados das simulações para cada porcentagem de verificação - dados de HIGGINS e KELLER (1973) - distribuições a priori informativas (amostra aleatória de $20 \%)$.

\begin{tabular}{|c|c|c|c|c|c|c|c|c|}
\hline \multicolumn{2}{|r|}{ Painel } & $\begin{array}{l}\text { Distribuição } \\
\text { a Priori }\end{array}$ & $\begin{array}{l}\text { Resultado } \\
\text { do Teste }\end{array}$ & \multicolumn{2}{|c|}{ Verificados pelo P.O. } & $\begin{array}{c}\text { Não } \\
\text { Verificados }\end{array}$ & $\begin{array}{r}\text { Estimativas } \\
\text { de B\&G }(\%)\end{array}$ & $\begin{array}{c}\text { Estimativas } \\
\text { Bayesianas (\%) }\end{array}$ \\
\hline & $\lambda=100 \%$ & - & Positivo & 65 & 134 & - & $\widehat{S_{E}}=20,25(10,48 ; 30,01)$ & - \\
\hline & & - & Negativo & 256 & 1409 & - & $\widehat{S_{P}}=91,32(89,85 ; 92,79)$ & - \\
\hline \multirow[t]{2}{*}{ (b) } & $\lambda=90 \%$ & $S_{E} \sim B(3,6 ; 9,4)$ & Positivo & 46 & 102 & 26 & $\widehat{S_{E}}=20,23(8,92 ; 31,55)$ & $\widehat{S_{E}}=19,93(12,81 ; 27,81)$ \\
\hline & & $S_{P} \sim B(81,9 ; 8,1)$ & Negativo & 191 & 996 & 138 & $\widehat{S_{P}}=90,26(88,48 ; 92,05)$ & $\widehat{S_{P}}=90,47(88,14 ; 92,56)$ \\
\hline \multirow[t]{2}{*}{ (c) } & $\lambda=80 \%$ & $S_{E} \sim B(3,6 ; 10,9)$ & Positivo & 45 & 91 & 30 & $\widehat{S_{E}}=21,35(9,67 ; 33,03)$ & $\widehat{S_{E}}=20,52(10,00 ; 30,89)$ \\
\hline & & $S_{P} \sim B(81,9 ; 8,1)$ & Negativo & 162 & 899 & 264 & $\widehat{S_{P}}=91,00(89,27 ; 92,73)$ & $\widehat{S_{P}}=91,05(88,34 ; 93,08)$ \\
\hline \multirow[t]{2}{*}{ (d) } & $\lambda=70 \%$ & $S_{E} \sim B(3,1 ; 6,8)$ & Positivo & 36 & 76 & 45 & $\widehat{S_{E}}=19,67(7,21 ; 32,13)$ & $\widehat{S_{E}}=19,10(7,36 ; 34,09)$ \\
\hline & & $S_{P} \sim B(57,2 ; 7,8)$ & Negativo & 144 & 788 & 402 & $\widehat{S_{P}}=91,37(89,63 ; 93,11)$ & $\widehat{S_{P}}=91,00(87,54 ; 93,92)$ \\
\hline \multirow[t]{2}{*}{ (e) } & $\lambda=60 \%$ & $S_{E} \sim B(2,9 ; 6,8)$ & Positivo & 32 & 63 & 65 & $\widehat{S_{E}}=20,51(7,40 ; 33,62)$ & $\widehat{S_{E}}=19,72(7,03 ; 37,65)$ \\
\hline & & $S_{P} \sim B(38,5 ; 5,8)$ & Negativo & 124 & 666 & 541 & $\widehat{S_{P}}=91,36(89,55 ; 93,17)$ & $\widehat{S_{P}}=90,92(86,52 ; 93,46)$ \\
\hline \multirow[t]{2}{*}{ (f) } & $\lambda=50 \%$ & $S_{E} \sim B(2,4 ; 4,5)$ & Positivo & 28 & 53 & 77 & $\widehat{S_{E}}=21,02(7,09 ; 34,95)$ & $\widehat{S_{E}}=21,00(6,03 ; 44,12)$ \\
\hline & & $S_{P} \sim B(22,2 ; 4,2)$ & Negativo & 105 & 577 & 651 & $\widehat{S_{P}}=91,60(89,75 ; 93,46)$ & $\widehat{S_{P}}=90,98(84,93 ; 95,25)$ \\
\hline \multirow[t]{2}{*}{ (g) } & $\lambda=40 \%$ & $S_{E} \sim B(2,2 ; 3,4)$ & Positivo & 20 & 43 & 93 & $\widehat{S_{E}}=19,46(3,64 ; 35,28)$ & $\widehat{S_{E}}=21,15(5,17 ; 51,97)$ \\
\hline & & $S_{P} \sim B(18,1 ; 4,0)$ & Negativo & 82 & 452 & 801 & $\widehat{S_{P}}=91,39(89,42 ; 93,36)$ & $\widehat{S_{P}}=90,76(83,44 ; 95,85)$ \\
\hline \multirow[t]{2}{*}{ (h) } & $\lambda=30 \%$ & $S_{E} \sim B(2,1 ; 3,0)$ & Positivo & 16 & 33 & 110 & $\widehat{S_{E}}=20,72(2,95 ; 38,48)$ & $\widehat{S_{E}}=22,60(3,16 ; 53,48)$ \\
\hline & & $S_{P} \sim B(10,3 ; 2,9)$ & Negativo & 61 & 348 & 923 & $\widehat{S_{P}}=91,37(89,24 ; 93,50)$ & $\widehat{S_{P}}=90,53(80,99 ; 96,45)$ \\
\hline \multirow[t]{2}{*}{ (i) } & $\lambda=20 \%$ & $S_{E} \sim B(1,7 ; 2,0)$ & Positivo & 11 & 20 & 125 & $\widehat{S_{E}}=21,36(1,34 ; 42,37)$ & $\widehat{S_{E}}=21,46(2,87 ; 64,21)$ \\
\hline & & $S_{P} \sim B(9,0 ; 3,0)$ & Negativo & 40 & 222 & 1073 & $\widehat{S_{P}}=91,83(89,37 ; 94,26)$ & $\widehat{S_{P}}=89,50(75,51 ; 96,09)$ \\
\hline \multirow[t]{2}{*}{ (j) } & $\lambda=10 \%$ & $S_{E} \sim B(1,6 ; 1,7)$ & Positivo & 5 & 10 & 141 & $\widehat{S_{E}}=19,52(0,59 ; 48,98)$ & $\widehat{S_{E}}=22,39(2,08 ; 73,23)$ \\
\hline & & $S_{P} \sim B(3,9 ; 2,3)$ & Negativo & 22 & 115 & 1198 & $\widehat{S_{P}}=91,51(88,25 ; 94,77)$ & $\widehat{S_{P}}=88,97(55,01 ; 96,75)$ \\
\hline
\end{tabular}

Tabela 30: Resultados das simulações para cada porcentagem de verificação - dados de HIGGINS e KELLER (1973) - distribuições a priori informativas (amostra aleatória de $30 \%)$.

\begin{tabular}{|c|c|c|c|c|c|c|c|c|}
\hline & Painel & $\begin{array}{c}\text { Distribuição } \\
\text { a Priori }\end{array}$ & $\begin{array}{c}\text { Resultado } \\
\text { do Teste }\end{array}$ & $\begin{array}{l}\text { Verificados } \\
\text { Positivo } \\
\end{array}$ & $\begin{array}{l}\text { pelo P.O. } \\
\text { Negativo }\end{array}$ & $\begin{array}{c}\text { Não } \\
\text { Verificados }\end{array}$ & $\begin{array}{c}\text { Estimativas } \\
\text { de B\&G (\%) }\end{array}$ & $\begin{array}{c}\text { Estimativas } \\
\text { Bayesianas (\%) }\end{array}$ \\
\hline & $\lambda=100 \%$ & - & Positivo & 65 & 134 & - & $\widehat{S_{E}}=20,25(10,48 ; 30,01)$ & - \\
\hline & & - & Negativo & 256 & 1409 & - & $\widehat{S_{P}}=91,32(89,85 ; 92,79)$ & - \\
\hline \multirow[t]{2}{*}{ (b) } & $\lambda=90 \%$ & $S_{E} \sim B(4,4 ; 13,3)$ & Positivo & 40 & 83 & 20 & $\widehat{S_{E}}=20,17(8,04 ; 32,29)$ & $\widehat{S_{E}}=19,33(12,64 ; 27,85)$ \\
\hline & & $S_{P} \sim B(95,9 ; 11,9)$ & Negativo & 166 & 888 & 115 & $\widehat{S_{P}}=91,08(89,25 ; 92,90)$ & $\widehat{S_{P}}=90,89(88,82 ; 92,94)$ \\
\hline \multirow[t]{2}{*}{ (c) } & $\lambda=80 \%$ & $S_{E} \sim B(4,1 ; 13,1)$ & Positivo & 37 & 74 & 31 & $\widehat{S_{E}}=20,14(8,02 ; 32,45)$ & $\widehat{S_{E}}=19,34(12,03 ; 27,53)$ \\
\hline & & $S_{P} \sim B(89,1 ; 9,9)$ & Negativo & 140 & 781 & 242 & $\widehat{S_{P}}=91,15(89,91 ; 32,21)$ & $\widehat{S_{P}}=90,90(88,72 ; 92,78)$ \\
\hline \multirow[t]{2}{*}{ (d) } & $\lambda=70 \%$ & $S_{E} \sim B(3,4 ; 9,3)$ & Positivo & 35 & 66 & 34 & $\widehat{S_{E}}=20,93(7,90 ; 33,96)$ & $\widehat{S_{E}}=20,00(8,91 ; 35,12)$ \\
\hline & & $S_{P} \sim B(57,2 ; 7,8)$ & Negativo & 124 & 697 & 349 & $\widehat{S_{P}}=91,84(90,05 ; 93,64)$ & $\widehat{S_{P}}=91,52(88,56 ; 94,00)$ \\
\hline \multirow[t]{2}{*}{ (e) } & $\lambda=60 \%$ & $S_{E} \sim B(3,2 ; 9,2)$ & Positivo & 29 & 61 & 60 & $\widehat{S_{E}}=22,11(7,87 ; 36,35)$ & $\widehat{S_{E}}=21,86(8,86 ; 39,71)$ \\
\hline & & $S_{P} \sim B(49,7 ; 5,5)$ & Negativo & 101 & 584 & 470 & $\widehat{S_{P}}=90,64(88,64 ; 92,64)$ & $\widehat{S_{P}}=90,72(86,53 ; 94,09)$ \\
\hline \multirow[t]{2}{*}{ (f) } & $\lambda=50 \%$ & $S_{E} \sim B(2,5 ; 5,1)$ & Positivo & 22 & 47 & 67 & $\widehat{S_{E}}=20,40(4,80 ; 36,00)$ & $\widehat{S_{E}}=19,43(5,20 ; 43,30)$ \\
\hline & & $S_{P} \sim B(29,3 ; 5,2)$ & Negativo & 87 & 514 & 568 & $\widehat{S_{P}}=91,52(89,55 ; 93,49)$ & $\widehat{S_{P}}=90,65(84,33 ; 94,92)$ \\
\hline \multirow[t]{2}{*}{ (g) } & $\lambda=40 \%$ & $S_{E} \sim B(2,3 ; 4,5)$ & Positivo & 19 & 38 & 78 & $\widehat{S_{E}}=21,46(4,53 ; 38,40)$ & $\widehat{S_{E}}=21,90(5,98 ; 48,23)$ \\
\hline & & $S_{P} \sim B(18,1 ; 4,0)$ & Negativo & 66 & 403 & 701 & $\widehat{S_{P}}=91,78(89,75 ; 93,82)$ & $\widehat{S_{P}}=90,92(83,03 ; 95,35)$ \\
\hline \multirow[t]{2}{*}{ (h) } & $\lambda=30 \%$ & $S_{E} \sim B(2,1 ; 3,0)$ & Positivo & 14 & 26 & 98 & $\widehat{S_{E}}=22,08(3,01 ; 41,16)$ & $\widehat{S_{E}}=21,50(3,77 ; 58,09)$ \\
\hline & & $S_{P} \sim B(10,3 ; 2,9)$ & Negativo & 53 & 310 & 804 & $\widehat{S_{P}}=91,74(89,44 ; 94,04)$ & $\widehat{S_{P}}=89,92(78,51 ; 96,80)$ \\
\hline \multirow[t]{2}{*}{ (i) } & $\lambda=20 \%$ & $S_{E} \sim B(1,8 ; 2,3)$ & Positivo & 11 & 21 & 118 & $\widehat{S_{E}}=22,13(1,20 ; 45,06)$ & $\widehat{S_{E}}=22,48(3,17 ; 66,25)$ \\
\hline & & $S_{P} \sim B(8,9 ; 2,8)$ & Negativo & 35 & 201 & 919 & $\widehat{S_{P}}=90,90(88,23 ; 93,57)$ & $\widehat{S_{P}}=89,08(76,17 ; 96,54)$ \\
\hline \multirow[t]{2}{*}{ (j) } & $\lambda=10 \%$ & $S_{E} \sim B(2,1 ; 2,5)$ & Positivo & 4 & 9 & 131 & $\widehat{S_{E}}=20,23(0,08 ; 54,02)$ & $\widehat{S_{E}}=21,78(5,79 ; 71,83)$ \\
\hline & & $S_{P} \sim B(7,7 ; 2,7)$ & Negativo & 17 & 96 & 1048 & $\widehat{S_{P}}=90,82(87,24 ; 94,40)$ & $\widehat{S_{P}}=89,27(75,27 ; 94,92)$ \\
\hline
\end{tabular}


Tabela 31: Resultados das simulações para cada porcentagem de verificação - dados de HIGGINS e KELLER (1973) - distribuições a priori informativas (amostra aleatória de $40 \%)$.

\begin{tabular}{|c|c|c|c|c|c|c|c|c|}
\hline & Painel & $\begin{array}{c}\text { Distribuição } \\
\text { a Priori }\end{array}$ & $\begin{array}{c}\text { Resultado } \\
\text { do Teste }\end{array}$ & $\begin{array}{l}\text { Verificados } \\
\text { Positivo } \\
\end{array}$ & $\begin{array}{l}\text { pelo P.O. } \\
\text { Negativo }\end{array}$ & $\begin{array}{c}\text { Não } \\
\text { Verificados }\end{array}$ & $\begin{array}{l}\text { Estimativas } \\
\text { de B\&G }(\%)\end{array}$ & $\begin{array}{c}\text { Estimativas } \\
\text { Bayesianas (\%) }\end{array}$ \\
\hline & $\lambda=100 \%$ & - & Positivo & 65 & 134 & - & $\widehat{S_{E}}=20,25(10,48 ; 30,01)$ & - \\
\hline & & - & Negativo & 256 & 1409 & - & $\widehat{S_{P}}=91,32(89,85 ; 92,79)$ & - \\
\hline \multirow[t]{2}{*}{ (b) } & $\lambda=90 \%$ & $S_{E} \sim B(5,2 ; 19,7)$ & Positivo & 40 & 71 & 19 & $\widehat{S_{E}}=22,02(10,35 ; 35,70)$ & $\widehat{S_{E}}=20,74(13,61 ; 29,17)$ \\
\hline & & $S_{P} \sim B(201,6 ; 22,4)$ & Negativo & 141 & 754 & 99 & $\widehat{S_{P}}=90,97(88,97 ; 92,96)$ & $\widehat{S_{P}}=90,59(88,63 ; 92,39)$ \\
\hline \multirow[t]{2}{*}{ (c) } & $\lambda=80 \%$ & $S_{E} \sim B(4,7 ; 13,5)$ & Positivo & 30 & 61 & 28 & $\widehat{S_{E}}=19,32(5,76 ; 32,88)$ & $\widehat{S_{E}}=19,11(10,43 ; 29,98)$ \\
\hline & & $S_{P} \sim B(95,9 ; 11,9)$ & Negativo & 133 & 678 & 188 & $\widehat{S_{P}}=91,28(89,28 ; 93,28)$ & $\widehat{S_{P}}=91,26(88,34 ; 94,00)$ \\
\hline \multirow[t]{2}{*}{ (d) } & $\lambda=70 \%$ & $S_{E} \sim B(3,9 ; 9,4)$ & Positivo & 22 & 57 & 38 & $\widehat{S_{E}}=19,09(2,07 ; 48,17)$ & $\widehat{S_{E}}=20,01 \quad(9,73 ; 33,86)$ \\
\hline & & $S_{P} \sim B(89,1 ; 9,9)$ & Negativo & 110 & 587 & 304 & $\widehat{S_{P}}=90,90(88,83 ; 92,96)$ & $\widehat{S_{P}}=91,30(88,32 ; 93,90)$ \\
\hline \multirow[t]{2}{*}{ (e) } & $\lambda=60 \%$ & $S_{E} \sim B(3,6 ; 10,9)$ & Positivo & 28 & 46 & 54 & $\widehat{S_{E}}=22,32(10,42 ; 32,79)$ & $\widehat{S_{E}}=23,94(13,53 ; 43,31)$ \\
\hline & & $S_{P} \sim B(60,6 ; 9,1)$ & Negativo & 85 & 506 & 99 & $\widehat{S_{P}}=88,13(85,22 ; 91,04)$ & $\widehat{S_{P}}=88,31(83,06 ; 92,04)$ \\
\hline \multirow[t]{2}{*}{ (f) } & $\lambda=50 \%$ & $S_{E} \sim B(3,7 ; 6,9)$ & Positivo & 19 & 41 & 58 & $\widehat{S_{E}}=21,32(12,10 ; 35,43)$ & $\widehat{S_{E}}=22,57(11,08 ; 39,13)$ \\
\hline & & $S_{P} \sim B(42,5 ; 7,5)$ & Negativo & 75 & 427 & 78 & $\widehat{S_{P}}=90,95(84,50 ; 93,68)$ & $\widehat{S_{P}}=88,37(80,98 ; 95,76)$ \\
\hline \multirow[t]{2}{*}{$(\mathrm{g})$} & $\lambda=40 \%$ & $S_{E} \sim B(2,5 ; 4,5)$ & Positivo & 12 & 31 & 69 & $\widehat{S_{E}}=18,00(5,26 ; 33 ; 17)$ & $\widehat{S_{E}}=22,33(5,75 ; 49,18)$ \\
\hline & & $S_{P} \sim B(23,1 ; 4,7)$ & Negativo & 57 & 334 & 615 & $\widehat{S_{P}}=91,41(89,17 ; 93,65)$ & $\widehat{S_{P}}=91,21(85,88 ; 95,82)$ \\
\hline \multirow[t]{2}{*}{ (h) } & $\lambda=30 \%$ & $S_{E} \sim B(1,9 ; 2,6)$ & Positivo & 11 & 26 & 64 & $\widehat{S_{E}}=17,10(2,32 ; 33,48)$ & $\widehat{S_{E}}=19,52(3,18 ; 49,23)$ \\
\hline & & $S_{P} \sim B(12,6 ; 3,6)$ & Negativo & 48 & 259 & 710 & $\widehat{S_{P}}=92,35(90,18 ; 94,54)$ & $\widehat{S_{P}}=91,11(80,00 ; 96,14)$ \\
\hline \multirow[t]{2}{*}{ (i) } & $\lambda=20 \%$ & $S_{E} \sim B(2,1 ; 3,0)$ & Positivo & 7 & 15 & 94 & $\widehat{S_{E}}=22,25(1,48 ; 41,23)$ & $\widehat{S_{E}}=23,12(4,64 ; 56,78)$ \\
\hline & & $S_{P} \sim B(9,0 ; 3,0)$ & Negativo & 26 & 176 & 800 & $\widehat{S_{P}}=91,69(88,92 ; 94,43)$ & $\widehat{S_{P}}=90,19(79,21 ; 95,49)$ \\
\hline \multirow[t]{2}{*}{$(\mathrm{j})$} & $\lambda=10 \%$ & $S_{E} \sim B(1,6 ; 1,7)$ & Positivo & 6 & 5 & 105 & $\widehat{S_{E}}=23,35(0,56 ; 46,28)$ & $\widehat{S_{E}}=22,68(3,98 ; 58,87)$ \\
\hline & & $S_{P} \sim B(4,5 ; 2,2)$ & Negativo & 20 & 82 & 900 & $\widehat{S_{P}}=91,85(88,79 ; 95,13)$ & $\widehat{S_{P}}=90,62(80,67 ; 97,19)$ \\
\hline
\end{tabular}

Para os dados do Exemplo 7 (BEGG e MCNEIL, 1988), foram feitas algumas simulações considerando diferentes probabilidades $-\lambda_{11}, \lambda_{10}, \lambda_{01}$ e $\lambda_{00}-$ para os indivíduos verificados, mas sempre atendendo o pressuposto utilizado por Begg e Greenes (1983). A partir do banco completo, as proporções foram sendo diminuídas, para que se pudesse avaliar o quanto o método bayesiano é eficiente na estimação da sensibilidade e da especificidade dos testes diagnósticos. Inicialmente, foram consideradas distribuições a priori não informativas. Já em um segundo momento, foram retiradas amostras aleatórias de $10 \%, 20 \%, 30 \%$ e $40 \%$ do banco completo, a partir das quais foram calculadas distribuições a priori informativas e feitas novas simulações com os dados restantes. Os resultados encontram-se nas Tabelas 32, 33, 34, 35 e 36 . 
Tabela 32: Resultados das simulações para cada porcentagem de verificação - dados de BEGG e MCNEIL (1988) - distribuições a priori não informativas.

\begin{tabular}{|c|c|c|c|c|c|c|c|}
\hline \multirow{2}{*}{\multicolumn{2}{|c|}{ Painel }} & \multirow{2}{*}{$\begin{array}{l}\text { Resultado } \\
\text { do Teste }\end{array}$} & \multicolumn{2}{|c|}{ Verificados pelo P.O. } & \multirow{2}{*}{$\begin{array}{c}\text { Não } \\
\text { Verificados }\end{array}$} & \multirow{2}{*}{$\begin{array}{r}\text { Estimativas } \\
\text { de B\&G }(\%) \\
\end{array}$} & \multirow{2}{*}{$\begin{array}{c}\text { Estimativas } \\
\text { Bayesianas (\%) }\end{array}$} \\
\hline & & & Positivo & Negativo & & & \\
\hline \multirow[t]{2}{*}{ (a) } & $\lambda=100 \%$ & Positivo & 302 & 80 & - & $\widehat{S_{E}}=62,79(57,33 ; 68,24)$ & - \\
\hline & & Negativo & 179 & 372 & - & $\widehat{S_{P}}=82,30(78,42 ; 86,17)$ & - \\
\hline \multirow[t]{2}{*}{ (b) } & $\lambda=90 \%$ & Positivo & 272 & 72 & 38 & $\widehat{S_{E}}=62,81(57,21 ; 68,41)$ & $\widehat{S_{E}}=60,72(54,16 ; 67,45)$ \\
\hline & & Negativo & 161 & 335 & 55 & $\widehat{S_{P}}=82,32(78,29 ; 86,34)$ & $\widehat{S_{P}}=80,00(73,85 ; 85,53)$ \\
\hline \multirow[t]{2}{*}{ (c) } & $\lambda=80 \%$ & Positivo & 242 & 64 & 76 & $\widehat{S_{E}}=62,84(57,06 ; 68,62)$ & $\widehat{S_{E}}=59,52(48,70 ; 69,92)$ \\
\hline & & Negativo & 143 & 298 & 110 & $\widehat{S_{P}}=82,33(78,13 ; 86,53)$ & $\widehat{S_{P}}=78,12(67,70 ; 87,04)$ \\
\hline \multirow[t]{2}{*}{ (d) } & $\lambda=70 \%$ & Positivo & 211 & 56 & 115 & $\widehat{S_{E}}=62,79(56,78 ; 68,80)$ & $\widehat{S_{E}}=57,72(42,80 ; 72,50)$ \\
\hline & & Negativo & 125 & 260 & 166 & $\widehat{S_{P}}=82,28(77,85 ; 86,71)$ & $\widehat{S_{P}}=76,29(61,38 ; 88,59)$ \\
\hline \multirow[t]{2}{*}{ (e) } & $\lambda=60 \%$ & Positivo & 181 & 48 & 153 & $\widehat{S_{E}}=62,83(56,52 ; 69,13)$ & $\widehat{S_{E}}=55,91(37,54 ; 75,82)$ \\
\hline & & Negativo & 107 & 223 & 221 & $\widehat{S_{P}}=82,30(77,60 ; 87,01)$ & $\widehat{S_{P}}=74,45(55,89 ; 89,83)$ \\
\hline \multirow[t]{2}{*}{ (f) } & $\lambda=50 \%$ & Positivo & 151 & 40 & 191 & $\widehat{S_{E}}=62,70(56,02 ; 69,38)$ & $\widehat{S_{E}}=54,67(31,52 ; 78,26)$ \\
\hline & & Negativo & 90 & 186 & 275 & $\widehat{S_{P}}=82,27(77,20 ; 87,35)$ & $\widehat{S_{P}}=72,71(48,34 ; 91,94)$ \\
\hline \multirow[t]{2}{*}{ (g) } & $\lambda=40 \%$ & Positivo & 121 & 32 & 229 & $\widehat{S_{E}}=62,73(55,52 ; 69,64)$ & $\widehat{S_{E}}=53,94(25,17 ; 81,92)$ \\
\hline & & Negativo & 72 & 149 & 330 & $\widehat{S_{P}}=82,30(76,73 ; 87,87)$ & $\widehat{S_{P}}=70,38(39,30 ; 93,33)$ \\
\hline \multirow[t]{2}{*}{ (h) } & $\lambda=30 \%$ & Positivo & 91 & 24 & 267 & $\widehat{S_{E}}=62,78(54,76 ; 70,79)$ & $\widehat{S_{E}}=52,23(17,46 ; 85,76)$ \\
\hline & & Negativo & 54 & 112 & 385 & $\widehat{S_{P}}=82,34(76,03 ; 88,65)$ & $\widehat{S_{P}}=67,27(30,89 ; 95,56)$ \\
\hline \multirow[t]{2}{*}{ (i) } & $\lambda=20 \%$ & Positivo & 60 & 16 & 306 & $\widehat{S_{E}}=62,58(53,11 ; 72,05)$ & $\widehat{S_{E}}=51,40(12,38 ; 89,98)$ \\
\hline & & Negativo & 36 & 74 & 441 & $\widehat{S_{P}}=82,17(74,53 ; 89,81)$ & $\widehat{S_{P}}=65,98(21,53 ; 96,53)$ \\
\hline \multirow{2}{*}{\multicolumn{2}{|c|}{ (j) $\lambda=10 \%$}} & Positivo & 60 & 8 & 344 & $\widehat{S_{E}}=62,57(49,76 ; 75,40)$ & $\widehat{S_{E}}=54,10(0,07 ; 94,61)$ \\
\hline & & Negativo & 18 & 37 & 496 & $\widehat{S_{P}}=82,17(71,57 ; 92,77)$ & $\widehat{S_{P}}=64,77(12,17 ; 98,26)$ \\
\hline
\end{tabular}

Tabela 33: Resultados das simulações para cada porcentagem de verificação - dados de BEGG e MCNEIL (1988) - distribuições a priori informativas (amostra aleatória de 10\%).

\begin{tabular}{|c|c|c|c|c|c|c|c|c|}
\hline & Painel & $\begin{array}{l}\text { Distribuição } \\
\text { a Priori }\end{array}$ & $\begin{array}{c}\text { Resultado } \\
\text { do Teste }\end{array}$ & $\begin{array}{l}\text { Verificad } \\
\text { Positivo } \\
\end{array}$ & $\begin{array}{l}\text { pelo P.O. } \\
\text { Negativo }\end{array}$ & $\begin{array}{c}\text { Não } \\
\text { Verificados }\end{array}$ & $\begin{array}{c}\text { Estimativas } \\
\text { de B\&G }(\%) \\
\end{array}$ & $\begin{array}{c}\text { Estimativas } \\
\text { Bayesianas (\%) }\end{array}$ \\
\hline \multirow[t]{2}{*}{ (a) } & $\lambda=100 \%$ & - & Positivo & 302 & 80 & - & $\widehat{S_{E}}=62,79(57,33 ; 68,24)$ & - \\
\hline & & - & Negativo & 179 & 372 & - & $\widehat{S_{P}}=82,30(78,42 ; 86,17)$ & - \\
\hline \multirow[t]{2}{*}{ (b) } & $\lambda=90 \%$ & $S_{E} \sim B(7,3 ; 6,5)$ & Positivo & 248 & 67 & 36 & $\widehat{S_{E}}=63,65(57,82 ; 69,48)$ & $\widehat{S_{E}}=61,40(54,37 ; 68,16)$ \\
\hline & & $S_{P} \sim B(15,0 ; 3,8)$ & Negativo & 142 & 298 & 49 & $\widehat{S_{P}}=81,60(77,27 ; 85,94)$ & $\widehat{S_{P}}=79,27(73,12 ; 85,16)$ \\
\hline \multirow[t]{2}{*}{ (c) } & $\lambda=80 \%$ & $S_{E} \sim B(6,6 ; 5,0)$ & Positivo & 216 & 60 & 66 & $\widehat{S_{E}}=62,87(56,72 ; 69,02)$ & $\widehat{S_{E}}=58,36(48,76 ; 68,58)$ \\
\hline & & $S_{P} \sim B(7,9 ; 3,1)$ & Negativo & 126 & 271 & 101 & $\widehat{S_{P}}=82,05(77,65 ; 86,46)$ & $\widehat{S_{P}}=77,45(69,11 ; 86,18)$ \\
\hline \multirow[t]{2}{*}{ (d) } & $\lambda=70 \%$ & $S_{E} \sim B(4,2 ; 3,4)$ & Positivo & 191 & 49 & 105 & $\widehat{S_{E}}=62,73(56,43 ; 69,04)$ & $\widehat{S_{E}}=56,80(43,56 ; 70,66)$ \\
\hline & & $S_{P} \sim B(7,9 ; 3,2)$ & Negativo & 114 & 232 & 149 & $\widehat{S_{P}}=82,49(77,81 ; 87,18)$ & $\widehat{S_{P}}=74,93(60,53 ; 87,31)$ \\
\hline \multirow[t]{2}{*}{ (e) } & $\lambda=60 \%$ & $S_{E} \sim B(3,9 ; 3,8)$ & Positivo & 164 & 43 & 141 & $\widehat{S_{E}}=65,11(58,55 ; 71,66)$ & $\widehat{S_{E}}=53,64(36,56 ; 74,62)$ \\
\hline & & $S_{P} \sim B(3,9 ; 2,5)$ & Negativo & 88 & 205 & 199 & $\widehat{S_{P}}=82,64(77,77 ; 87,51)$ & $\widehat{S_{P}}=70,15(53,79 ; 87,07)$ \\
\hline \multirow[t]{2}{*}{ (f) } & $\lambda=50 \%$ & $S_{E} \sim B(3,2 ; 2,6)$ & Positivo & 132 & 34 & 174 & $\widehat{S_{E}}=62,80(55,77 ; 69,82)$ & $\widehat{S_{E}}=51,93(30,47 ; 74,42)$ \\
\hline & & $S_{P} \sim B(2,9 ; 2,3)$ & Negativo & 82 & 174 & 244 & $\widehat{S_{P}}=82,99(77,72 ; 88,27)$ & $\widehat{S_{P}}=69,69(48,54 ; 89,00)$ \\
\hline \multirow[t]{2}{*}{ (g) } & $\lambda=40 \%$ & $S_{E} \sim B(2,8 ; 2,4)$ & Positivo & 106 & 27 & 209 & $\widehat{S_{E}}=63,43(55,91 ; 70,96)$ & $\widehat{S_{E}}=50,05(26,33 ; 77,57)$ \\
\hline & & $S_{P} \sim B(2,2 ; 1,9)$ & Negativo & 65 & 141 & 292 & $\widehat{S_{P}}=83,08(77,29 ; 88,87)$ & $\widehat{S_{P}}=67,73(41,23 ; 90,23)$ \\
\hline \multirow[t]{2}{*}{ (h) } & $\lambda=30 \%$ & $S_{E} \sim B(1,9 ; 1,8)$ & Positivo & 83 & 21 & 239 & $\widehat{S_{E}}=62,46(53,97 ; 70,94)$ & $\widehat{S_{E}}=49,98(22,29 ; 80,30)$ \\
\hline & & $S_{P} \sim B(2,6 ; 1,9)$ & Negativo & 48 & 97 & 352 & $\widehat{S_{P}}=82,76(76,14 ; 89,38)$ & $\widehat{S_{P}}=66,25(34,03 ; 92,56)$ \\
\hline \multirow[t]{2}{*}{ (i) } & $\lambda=20 \%$ & $S_{E} \sim B(1,9 ; 1,9)$ & Positivo & 52 & 15 & 272 & $\widehat{S_{E}}=63,48(53,07 ; 73,89)$ & $\widehat{S_{E}}=47,85(18,30 ; 81,98)$ \\
\hline & & $S_{P} \sim B(2,0 ; 1,7)$ & Negativo & 29 & 67 & 405 & $\widehat{S_{P}}=82,17(74,33 ; 90,00)$ & $\widehat{S_{P}}=62,89(26,71 ; 92,15)$ \\
\hline \multirow[t]{2}{*}{ (j) } & $\lambda=10 \%$ & $S_{E} \sim B(1,5 ; 1,5)$ & Positivo & 27 & 8 & 312 & $\widehat{S_{E}}=62,86(49,54 ; 76,19)$ & $\widehat{S_{E}}=47,35(12,02 ; 87,59)$ \\
\hline & & $S_{P} \sim B(1,5 ; 1,5)$ & Negativo & 17 & 36 & 440 & $\widetilde{S_{P}}=80,85(69,64 ; 92,06)$ & $\widehat{S_{P}}=61,01(17,75 ; 94,50)$ \\
\hline
\end{tabular}


Tabela 34: Resultados das simulações para cada porcentagem de verificação - dados de BEGG e MCNEIL (1988) - distribuições a priori informativas (amostra aleatória de 20\%).

\begin{tabular}{|c|c|c|c|c|c|c|c|c|}
\hline & Painel & $\begin{array}{c}\text { Distribuição } \\
\text { a Priori }\end{array}$ & $\begin{array}{c}\text { Resultado } \\
\text { do Teste }\end{array}$ & $\begin{array}{l}\text { Verificados } \\
\text { Positivo } \\
\end{array}$ & $\begin{array}{l}\text { pelo P.O. } \\
\text { Negativo }\end{array}$ & $\begin{array}{c}\text { Não } \\
\text { Verificados }\end{array}$ & $\begin{array}{l}\text { Estimativas } \\
\text { de B\&G }(\%)\end{array}$ & $\begin{array}{c}\text { Estimativas } \\
\text { Bayesianas (\%) }\end{array}$ \\
\hline (a) & $\lambda=100 \%$ & $\begin{array}{l}- \\
-\end{array}$ & $\begin{array}{l}\text { Positivo } \\
\text { Negativo }\end{array}$ & $\begin{array}{l}302 \\
179\end{array}$ & $\begin{array}{c}80 \\
372\end{array}$ & $\begin{array}{l}- \\
-\end{array}$ & $\begin{array}{l}\widehat{S_{E}}=62,79(57,33 ; 68,24) \\
\widehat{S_{P}}=82,30(78,42 ; 86,17)\end{array}$ & $\begin{array}{l}- \\
-\end{array}$ \\
\hline (b) & $\lambda=90 \%$ & $\begin{array}{l}S_{E} \sim B(13,9 ; 8,9) \\
S_{P} \sim B(20,5 ; 6,1)\end{array}$ & $\begin{array}{l}\text { Positivo } \\
\text { Negativo }\end{array}$ & $\begin{array}{l}216 \\
130\end{array}$ & $\begin{array}{c}58 \\
275\end{array}$ & $\begin{array}{l}30 \\
37\end{array}$ & $\begin{aligned} \widehat{S_{E}} & =62,81(56,55 ; 69,08) \\
\widehat{S_{P}} & =82,34(77,87 ; 86,82)\end{aligned}$ & $\begin{array}{l}\widehat{\widehat{S_{E}}}=61,04(54,70 ; 67,18) \\
\widehat{S_{P}}=80,04(74,53 ; 85,50)\end{array}$ \\
\hline (c) & $\lambda=80 \%$ & $\begin{array}{l}S_{E} \sim B(9,5 ; 5,8) \\
S_{P} \sim B(9,5 ; 4,1)\end{array}$ & $\begin{array}{l}\text { Positivo } \\
\text { Negativo }\end{array}$ & $\begin{array}{l}189 \\
118\end{array}$ & $\begin{array}{c}50 \\
245\end{array}$ & $\begin{array}{l}60 \\
84\end{array}$ & $\begin{array}{l}\widehat{S_{E}}=61,94(55,40 ; 68,48) \\
\widehat{S_{P}}=82,83(78,21 ; 87,44)\end{array}$ & $\begin{array}{l}\widehat{S_{E}}=58,82(48,96 ; 69,15) \\
\widehat{S_{P}}=78,33 \quad(69,32 ; 86,96)\end{array}$ \\
\hline (d) & $\lambda=70 \%$ & $\begin{array}{l}S_{E} \sim B(7,5 ; 6,1) \\
S_{P} \sim B(7,8 ; 2,9)\end{array}$ & $\begin{array}{l}\text { Positivo } \\
\text { Negativo }\end{array}$ & $\begin{array}{c}169 \\
94\end{array}$ & $\begin{array}{c}49 \\
209\end{array}$ & $\begin{array}{c}88 \\
137\end{array}$ & $\begin{array}{l}\widehat{S_{E}}=63,48(56,68 ; 70,27) \\
\widehat{S_{P}}=81,52(76,58 ; 86,47)\end{array}$ & $\begin{array}{l}\widehat{S_{E}}=57,88(44,72 ; 72,56) \\
\widehat{S_{P}}=75,93(63,25 ; 86,66)\end{array}$ \\
\hline (e) & $\lambda=60 \%$ & $\begin{array}{l}S_{E} \sim B(4,8 ; 3,8) \\
S_{P} \sim B(6,8 ; 2,9)\end{array}$ & $\begin{array}{l}\text { Positivo } \\
\text { Negativo }\end{array}$ & $\begin{array}{c}140 \\
85\end{array}$ & $\begin{array}{c}41 \\
178\end{array}$ & $\begin{array}{l}130 \\
172\end{array}$ & $\begin{array}{l}\widehat{S_{E}}=61,68(54,26 ; 69,09) \\
\widehat{S_{P}}=81,63(76,29 ; 86,96)\end{array}$ & $\begin{array}{l}\widehat{S_{E}}=55,95(40,82 ; 70,31) \\
\widehat{S_{P}}=74,67 \quad(60,52 ; 87,20)\end{array}$ \\
\hline (f) & $\lambda=50 \%$ & $\begin{array}{l}S_{E} \sim B(4,6 ; 4,1) \\
S_{P} \sim B(5,1 ; 2,8)\end{array}$ & $\begin{array}{l}\text { Positivo } \\
\text { Negativo }\end{array}$ & $\begin{array}{c}119 \\
70\end{array}$ & $\begin{array}{c}30 \\
148\end{array}$ & $\begin{array}{l}159 \\
220\end{array}$ & $\begin{array}{l}\widehat{S_{E}}=63,62(56,23 ; 71,02) \\
\widehat{S_{P}}=82,74(77,04 ; 88,44)\end{array}$ & $\begin{aligned} \widehat{S_{E}} & =53,90(33,52 ; 75,83) \\
\widehat{S_{P}} & =69,78(47,41 ; 90,13)\end{aligned}$ \\
\hline (g) & $\lambda=40 \%$ & $\begin{array}{l}S_{E} \sim B(2,8 ; 2,5) \\
S_{P} \sim B(2,6 ; 2,0)\end{array}$ & $\begin{array}{l}\text { Positivo } \\
\text { Negativo }\end{array}$ & $\begin{array}{l}97 \\
59\end{array}$ & $\begin{array}{c}26 \\
129\end{array}$ & $\begin{array}{l}183 \\
252\end{array}$ & $\begin{array}{l}\widehat{S_{E}}=63,60(55,69 ; 71,52) \\
\widehat{S_{P}}=82,36(76,21 ; 88,50)\end{array}$ & $\begin{array}{l}\widehat{S_{E}}=51,55(28,33 ; 79,20) \\
\widehat{S_{P}}=69,46(44,61 ; 91,34)\end{array}$ \\
\hline$(\mathrm{h})$ & $\lambda=30 \%$ & $\begin{array}{l}S_{E} \sim B(2,1 ; 2,1) \\
S_{P} \sim B(3,0 ; 2,0)\end{array}$ & $\begin{array}{l}\text { Positivo } \\
\text { Negativo }\end{array}$ & $\begin{array}{l}77 \\
44\end{array}$ & $\begin{array}{l}20 \\
87\end{array}$ & $\begin{array}{l}214 \\
304\end{array}$ & $\begin{array}{l}\widehat{S_{E}}=62,82(54,01 ; 71,63) \\
\widehat{S_{P}}=81,84(74,70 ; 88,97)\end{array}$ & $\begin{array}{l}\widehat{S_{E}}=49,64(23,07 ; 79,34) \\
\widehat{S_{P}}=64,78(36,13 ; 90,56)\end{array}$ \\
\hline (i) & $\lambda=20 \%$ & $\begin{array}{l}S_{E} \sim B(2,2 ; 2,0) \\
S_{P} \sim B(2,0 ; 1,7)\end{array}$ & $\begin{array}{l}\text { Positivo } \\
\text { Negativo }\end{array}$ & $\begin{array}{l}47 \\
29\end{array}$ & $\begin{array}{l}12 \\
59\end{array}$ & $\begin{array}{l}233 \\
366\end{array}$ & $\begin{array}{l}\widehat{S_{E}}=60,86(49,97 ; 71,74) \\
\widehat{S_{P}}=83,67(75,52 ; 91,83)\end{array}$ & $\begin{aligned} \widehat{S_{E}} & =48,60(17,71 ; 80,24) \\
\widehat{S_{P}} & =64,36(32,37 ; 91,98)\end{aligned}$ \\
\hline (j) & $\lambda=10 \%$ & $\begin{array}{l}S_{E} \sim B(2,0 ; 1,7) \\
S_{P} \sim B(1,8 ; 1,5)\end{array}$ & $\begin{array}{l}\text { Positivo } \\
\text { Negativo }\end{array}$ & $\begin{array}{l}23 \\
16 \\
\end{array}$ & $\begin{array}{c}16 \\
8\end{array}$ & $\begin{array}{l}276 \\
397 \\
\end{array}$ & $\begin{array}{l}\widehat{S_{E}}=61,78(45,62 ; 73,12) \\
\widehat{S_{P}}=82,63(74,59 ; 90,47)\end{array}$ & $\begin{aligned} \widehat{S_{E}} & =48,49(20,58 ; 88,28) \\
\widehat{S_{P}} & =55,25(15,95 ; 89,82)\end{aligned}$ \\
\hline
\end{tabular}

Tabela 35: Resultados das simulações para cada porcentagem de verificação - dados de BEGG e MCNEIL (1988) - distribuições a priori informativas (amostra aleatória de 30\%).

\begin{tabular}{|c|c|c|c|c|c|c|c|c|}
\hline \multicolumn{2}{|r|}{ Painel } & $\begin{array}{l}\text { Distribuição } \\
\text { a Priori }\end{array}$ & $\begin{array}{l}\text { Resultado } \\
\text { do Teste }\end{array}$ & \multicolumn{2}{|c|}{ Verificados pelo P.O. } & $\begin{array}{c}\text { Não } \\
\text { Verificados }\end{array}$ & $\begin{array}{l}\text { Estimativas } \\
\text { de B\&G }(\%)\end{array}$ & $\begin{array}{c}\text { Estimativas } \\
\text { Bayesianas (\%) }\end{array}$ \\
\hline & $\lambda=100 \%$ & - & Positivo & 302 & 80 & - & $\widehat{S_{E}}=62,79(57,33 ; 68,24)$ & - \\
\hline & & - & Negativo & 179 & 372 & - & $\widehat{S_{P}}=82,30(78,42 ; 86,17)$ & - \\
\hline \multirow[t]{2}{*}{ (b) } & $\lambda=90 \%$ & $S_{E} \sim B(53,9 ; 44,1)$ & Positivo & 193 & 50 & 29 & $\widehat{S_{E}}=64,14(57,58 ; 70,70)$ & $\widehat{S_{E}}=60,00(54,42 ; 65,36)$ \\
\hline & & $S_{P} \sim B(31,7 ; 6,5)$ & Negativo & 110 & 237 & 34 & $\widehat{S_{P}}=82,30(77,47 ; 87,13)$ & $\widehat{S_{P}}=79,86(73,56 ; 85,73)$ \\
\hline \multirow[t]{2}{*}{ (c) } & $\lambda=80 \%$ & $S_{E} \sim B(21,5 ; 15,6)$ & Positivo & 198 & 52 & 24 & $\widehat{S_{E}}=64,18(57,61 ; 70,75)$ & $\widehat{S_{E}}=61,58(55,13 ; 68,03)$ \\
\hline & & $S_{P} \sim B(26,0 ; 6,9)$ & Negativo & 108 & 230 & 41 & $\widehat{S_{P}}=81,90(77,04 ; 86,76)$ & $\widehat{S_{P}}=79,35(73,61 ; 84,66)$ \\
\hline \multirow[t]{2}{*}{ (d) } & $\lambda=70 \%$ & $S_{E} \sim B(7,9 ; 5,3)$ & Positivo & 143 & 42 & 79 & $\widehat{S_{E}}=60,97(53,62 ; 68,33)$ & $\widehat{S_{E}}=56,61(43,27 ; 69,52)$ \\
\hline & & $S_{P} \sim B(11,2 ; 4,1)$ & Negativo & 92 & 182 & 115 & $\widehat{S_{P}}=81,17(75,75 ; 86,59)$ & $\widehat{S_{P}}=75,23(63,40 ; 86,23)$ \\
\hline \multirow[t]{2}{*}{ (e) } & $\lambda=60 \%$ & $S_{E} \sim B(5,4 ; 4,6)$ & Positivo & 131 & 35 & 106 & $\widehat{S_{E}}=64,08(56,64 ; 71,52)$ & $\widehat{S_{E}}=55,52(39,62 ; 73,56)$ \\
\hline & & $S_{P} \sim B(6,8 ; 2,9)$ & Negativo & 72 & 156 & 153 & $\widehat{S_{P}}=81,97(76,35 ; 87,59)$ & $\widehat{S_{P}}=73,12(56,78 ; 87,87)$ \\
\hline \multirow[t]{2}{*}{ (f) } & $\lambda=50 \%$ & $S_{E} \sim B(4,7 ; 4,0)$ & Positivo & 104 & 26 & 136 & $\widehat{S_{E}}=62,13(54,07 ; 70,19)$ & $\widehat{S_{E}}=52,69(33,58 ; 74,37)$ \\
\hline & & $S_{P} \sim B(5,9 ; 3,2)$ & Negativo & 62 & 123 & 202 & $\widehat{S_{P}}=82,87(76,77 ; 88,97)$ & $\widehat{S_{P}}=70,94(51,87 ; 90,19)$ \\
\hline \multirow[t]{2}{*}{ (g) } & $\lambda=40 \%$ & $S_{E} \sim B(3,7 ; 3,0)$ & Positivo & 81 & 25 & 155 & $\widehat{S_{E}}=61,65(52,70 ; 70,60)$ & $\widehat{S_{E}}=51,15(29,29 ; 75,76)$ \\
\hline & & $S_{P} \sim B(3,9 ; 2,5)$ & Negativo & 50 & 108 & 234 & $\widehat{S_{P}}=81,32(74,72 ; 87,91)$ & $\widehat{S_{P}}=68,74(46,13 ; 89,84)$ \\
\hline \multirow[t]{2}{*}{ (h) } & $\lambda=30 \%$ & $S_{E} \sim B(2,3 ; 2,4)$ & Positivo & 67 & 19 & 199 & $\widehat{S_{E}}=63,38(55,15 ; 73,63)$ & $\widehat{S_{E}}=52,18(24,08 ; 80,66)$ \\
\hline & & $S_{P} \sim B(3,4 ; 2,3)$ & Negativo & 33 & 80 & 255 & $\widehat{S_{P}}=80,53(72,81 ; 88,26)$ & $\widehat{S_{P}}=63,84(38,65 ; 89,76)$ \\
\hline \multirow[t]{2}{*}{ (i) } & $\lambda=20 \%$ & $S_{E} \sim B(1,9 ; 1,8)$ & Positivo & 42 & 10 & 215 & $\widehat{S_{E}}=60,81(49,67 ; 71,96)$ & $\widehat{S_{E}}=49,26(22,47 ; 82,55)$ \\
\hline & & $S_{P} \sim B(2,6 ; 2,0)$ & Negativo & 27 & 48 & 308 & $\widehat{S_{P}}=82,79(73,35 ; 92,23)$ & $\widehat{S_{P}}=65,47(33,81 ; 92,30)$ \\
\hline \multirow[t]{2}{*}{ (j) } & $\lambda=10 \%$ & $S_{E} \sim B(1,7 ; 1,7)$ & Positivo & 22 & 6 & 246 & $\widehat{S_{E}}=62,36(46,86 ; 77,86)$ & $\widehat{S_{E}}=48,02(18,06 ; 85,23)$ \\
\hline & & $S_{P} \sim B(2,0 ; 1,7)$ & Negativo & 12 & 23 & 344 & $\widehat{S_{P}}=80,92(67,80 ; 94,04)$ & $\widehat{S_{P}}=62,07(21,96 ; 92,92)$ \\
\hline
\end{tabular}


Tabela 36: Resultados das simulações para cada porcentagem de verificação - dados de BEGG e MCNEIL (1988) - distribuições a priori informativas (amostra aleatória de 40\%).

\begin{tabular}{|c|c|c|c|c|c|c|c|}
\hline Painel & $\begin{array}{l}\text { Distribuição } \\
\text { a Priori } \\
\end{array}$ & $\begin{array}{l}\text { Resultado } \\
\text { do Teste }\end{array}$ & $\begin{array}{l}\text { Verificados } \\
\text { Positivo } \\
\end{array}$ & $\begin{array}{l}\text { pelo P.O. } \\
\text { Negativo }\end{array}$ & $\begin{array}{c}\text { Não } \\
\text { Verificados } \\
\end{array}$ & $\begin{array}{c}\text { Estimativas } \\
\text { de B\&G }(\%) \\
\end{array}$ & $\begin{array}{c}\text { Estimativas } \\
\text { Bayesianas (\%) }\end{array}$ \\
\hline \multirow[t]{2}{*}{ (a) $\lambda=100 \%$} & - & Positivo & 302 & 80 & - & $\widehat{S_{E}}=62,79(57,33 ; 68,24)$ & - \\
\hline & - & Negativo & 179 & 372 & - & $\widehat{S_{P}}=82,30(78,42 ; 86,17)$ & - \\
\hline \multirow[t]{2}{*}{ (b) $\lambda=90 \%$} & $S_{E} \sim B(21,0 ; 16,5)$ & Positivo & 160 & 50 & 30 & $\widehat{S_{E}}=63,38(56,43 ; 70,34)$ & $\widehat{S_{E}}=63,15(56,08 ; 70,12)$ \\
\hline & $S_{P} \sim B(27,5 ; 8,7)$ & Negativo & 90 & 210 & 39 & $\widehat{S_{P}}=80,49(75,12 ; 86,05)$ & $\widehat{S_{P}}=79,57(72,46 ; 85,89)$ \\
\hline \multirow[t]{2}{*}{ (c) $\lambda=80 \%$} & $S_{E} \sim B(17,3 ; 11,1)$ & Positivo & 138 & 41 & 41 & $\widehat{S_{E}}=61,16(53,42 ; 68,91)$ & $\widehat{S_{E}}=58,51(48,74 ; 67,46)$ \\
\hline & $S_{P} \sim B(16,6 ; 5,5)$ & Negativo & 88 & 198 & 64 & $\widehat{S_{P}}=82,78(77,67 ; 87,90)$ & $\widehat{S_{P}}=78,63(70,43 ; 86,23)$ \\
\hline \multirow[t]{2}{*}{ (d) $\lambda=70 \%$} & $S_{E} \sim B(9,2 ; 6,7)$ & Positivo & 129 & 34 & 69 & $\widehat{S_{E}}=61,88(54,18 ; 69,58)$ & $\widehat{S_{E}}=57,69(45,64 ; 70,04)$ \\
\hline & $S_{P} \sim B(13,5 ; 4,7)$ & Negativo & 80 & 152 & 96 & $\widehat{S_{P}}=81,62(75,71 ; 87,52)$ & $\widehat{S_{P}}=75,50(62,85 ; 86,76)$ \\
\hline \multirow[t]{2}{*}{ (e) $\lambda=60 \%$} & $S_{E} \sim B(7,7 ; 5,8)$ & Positivo & 106 & 24 & 94 & $\widehat{S_{E}}=63,50(55,31 ; 71,69)$ & $\widehat{S_{E}}=55,00(39,40 ; 72,41)$ \\
\hline & $S_{P} \sim B(8,1 ; 3,8)$ & Negativo & 60 & 132 & 144 & $\widehat{S_{P}}=83,82(78,08 ; 89,85)$ & $\widehat{S_{P}}=73,94(58,75 ; 89,31)$ \\
\hline \multirow[t]{2}{*}{ (f) $\lambda=50 \%$} & $S_{E} \sim B(4,2 ; 3,3)$ & Positivo & 96 & 30 & 100 & $\widehat{S_{E}}=57,89(49,05 ; 66,73)$ & $\widehat{S_{E}}=53,10(34,86 ; 73,09)$ \\
\hline & $S_{P} \sim B(5,9 ; 2,9)$ & Negativo & 63 & 105 & 166 & $\widehat{S_{P}}=79,51(72,67 ; 86,34)$ & $\widehat{S_{P}}=71,72 \quad(53,05 ; 87,95)$ \\
\hline \multirow{2}{*}{ (g) $\lambda=40 \%$} & $S_{E} \sim B(3,2 ; 2,7)$ & Positivo & 72 & 19 & 133 & $\widehat{S_{E}}=62,52(53,11 ; 71,93)$ & $\widehat{S_{E}}=51,99(27,72 ; 78,12)$ \\
\hline & $S_{P} \sim B(3,9 ; 2,4)$ & Negativo & 43 & 93 & 200 & $\widehat{S_{P}}=83,09(76,16 ; 90,00)$ & $\widehat{S_{P}}=70,12(45,58 ; 90,39)$ \\
\hline \multirow[t]{2}{*}{ (h) $\lambda=30 \%$} & $S_{E} \sim B(2,7 ; 2,5)$ & Positivo & 53 & 10 & 169 & $\widehat{S_{E}}=65,52(55,66 ; 75,38)$ & $\widehat{S_{E}}=50,72(22,80 ; 80,19)$ \\
\hline & $S_{P} \sim B(3,8 ; 2,6)$ & Negativo & 31 & 68 & 229 & $\widehat{S_{P}}=85,95(78,04 ; 93,86)$ & $\widehat{S_{P}}=65,11(39,21 ; 90,40)$ \\
\hline \multirow[t]{2}{*}{ (i) $\lambda=20 \%$} & $S_{E} \sim B(2,0 ; 2,1)$ & Positivo & 40 & 11 & 188 & $\widehat{S_{E}}=66,69(55,20 ; 78,18)$ & $\widehat{S_{E}}=47,19(19,48 ; 81,87)$ \\
\hline & $S_{P} \sim B(2,2 ; 1,9)$ & Negativo & 21 & 51 & 249 & $\widehat{S_{P}}=81,52(72,02 ; 91,02)$ & $\widehat{S_{P}}=59,67(28,77 ; 88,04)$ \\
\hline \multirow[t]{2}{*}{ (j) $\lambda=10 \%$} & $S_{E} \sim B(1,7 ; 1,6)$ & Positivo & 18 & 5 & 209 & $\widehat{S_{E}}=62,42(45,95 ; 78,88)$ & $\widehat{S_{E}}=46,58(13,77 ; 87,52)$ \\
\hline & $S_{P} \sim B(1,8 ; 1,6)$ & Negativo & 11 & 22 & 295 & $\widehat{S_{P}}=81,26(67,25 ; 95,27)$ & $\widehat{S_{P}}=60,86(17,27 ; 92,88)$ \\
\hline
\end{tabular}

\subsubsection{Diferentes Probabilidades de Verificação dos Indivíduos}

Considerando-se os dados dos mesmos exemplos já utilizados anteriormente, simulações foram realizadas. Agora, o pressuposto de Begg e Greenes (1983) não foi atendido. Foram propostos diferentes valores para as probabilidades de verificação dos indivíduos e comparados os resultados do método bayesiano com os obtidos a partir da metodologia de Begg e Greenes (1983).

Para os dados do Exemplo 1 (WEINER et al., 1979), os resultados são dados nas Tabelas 37, 38, 39 e 40 .

Já para o os dados do Exemplo 2 (HIGGINS e KELLER, 1973), os resultados são dados nas Tabelas 41, 42, 43 e 44.

Para os dados do Exemplo 7 (BEGG e MCNEIL, 1988), os resultados são dados nas Tabelas 45, 46, 47 e 48. 
Tabela 37: Resultados das simulações para cada porcentagem de verificação - dados de WEINER et al. (1979) - distribuições a priori não informativas (diferentes probabilidades de verificação).

\begin{tabular}{|c|c|c|c|c|c|c|}
\hline Painel & $\begin{array}{l}\text { Resultado } \\
\text { do Teste }\end{array}$ & \multicolumn{2}{|c|}{ Verificados pelo P. O. } & $\begin{array}{c}\text { Não } \\
\text { Verificados }\end{array}$ & $\begin{array}{c}\text { Estimativas } \\
\text { de B\&G }(\%)\end{array}$ & $\begin{array}{c}\text { Estimativas } \\
\text { Bayesianas (\%) }\end{array}$ \\
\hline$\lambda_{01}=\lambda_{10}=100 \%$ & Negativo & 208 & 327 & - & $\widehat{S_{P}}=73,98(69,23 ; 78,74)$ & - \\
\hline$\lambda_{01}=\lambda_{10}=90 \%$ & Negativo & 187 & 327 & 21 & $\widehat{S_{P}}=76,38(71,84 ; 80,92)$ & $\widehat{S_{P}}=75,60(71,52 ; 79,92)$ \\
\hline (c) $\lambda_{11}=\lambda_{00}=100 \%$ & Positivo & 815 & 92 & 23 & $\widehat{S_{E}}=82,27(79,62 ; 84,92)$ & $\widehat{S_{E}}=81,58(78,42 ; 84,62)$ \\
\hline$\lambda_{01}=\lambda_{10}=70 \%$ & Negativo & 146 & 327 & 62 & $\widehat{S_{P}}=81,48(77,45 ; 85,50)$ & $\widehat{S_{P}}=78,47(72,70 ; 83,81)$ \\
\hline (e) $\lambda_{11}=\lambda_{00}=100 \%$ & Positivo & 815 & 69 & 46 & $\widehat{S_{E}}=85,28(82,78 ; 87,79)$ & $\widehat{S_{E}}=83,73(78,37 ; 88,25)$ \\
\hline$\lambda_{01}=\lambda_{10}=60 \%$ & Negativo & 125 & 327 & 83 & $\widehat{S_{P}}=84,21(80,49 ; 87,93)$ & $\widehat{S_{P}}=80,40(73,23 ; 86,72)$ \\
\hline (f) $\lambda_{11}=\lambda_{00}=100 \%$ & Positivo & 815 & 58 & 57 & $\widehat{S_{E}}=87,06(84,65 ; 89,46)$ & $\widehat{S_{E}}=84,84(79,32 ; 90,00)$ \\
\hline (h) $\lambda_{11}=\lambda_{00}=100 \%$ & Positivo & 815 & 34 & 81 & $\widehat{S_{E}}=91,28(89,18 ; 93,38)$ & $\widehat{S_{E}}=87,31(79,46 ; 93,99)$ \\
\hline$\lambda_{01}=\lambda_{10}=30 \%$ & Negativo & 62 & 327 & 146 & $\widehat{S_{P}}=92,35(89,79 ; 94,92)$ & $\widetilde{S_{P}}=85,65(74,82 ; 93,64)$ \\
\hline (i) $\lambda_{11}=\lambda_{00}=100 \%$ & Positivo & 815 & 23 & 92 & $\widehat{S_{E}}=93,69(91,84 ; 95,54)$ & $\widehat{S_{E}}=88,94(79,49 ; 95,88)$ \\
\hline$\lambda_{01}=\lambda_{10}=20 \%$ & Negativo & 42 & 327 & 166 & $\widehat{S_{P}}=94,89(92,81 ; 96,97)$ & $\widehat{S_{P}}=88,20(75,51 ; 96,06)$ \\
\hline (j) $\lambda_{11}=\lambda_{00}=100 \%$ & Positivo & 815 & 11 & 104 & $\widehat{S_{E}}=96,60(95,17 ; 98,03)$ & $\widehat{S_{E}}=90,48(79,79 ; 98,01)$ \\
\hline$\lambda_{01}=\lambda_{10}=10 \%$ & Negativo & 21 & 327 & 187 & $\widehat{S_{P}}=97,60(96,18,99,01)$ & $\widehat{S_{P}}=90,40(75,77 ; 98,22)$ \\
\hline
\end{tabular}

Tabela 38: Resultados das simulações para cada porcentagem de verificação - dados de WEINER et al. (1979) - distribuições a priori não informativas (diferentes probabilidades de verificação).

\begin{tabular}{|c|c|c|c|c|c|c|}
\hline Painel & $\begin{array}{l}\text { Resultado } \\
\text { do Teste }\end{array}$ & $\begin{array}{l}\text { Verificado } \\
\text { Positivo } \\
\end{array}$ & $\frac{\text { pelo P. O. }}{\text { Negativo }}$ & $\begin{array}{c}\text { Não } \\
\text { Verificados }\end{array}$ & $\begin{array}{c}\text { Estimativas } \\
\text { de B\&G }(\%)\end{array}$ & $\begin{array}{c}\text { Estimativas } \\
\text { Bayesianas (\%) }\end{array}$ \\
\hline$\lambda_{01}=\lambda_{10}=100 \%$ & Negativo & 208 & 327 & - & $\widehat{S_{P}}=73,98(69,23 ; 78,74)$ & - \\
\hline$\lambda_{01}=\lambda_{10}=90 \%$ & Negativo & 187 & 262 & 86 & $\widehat{S_{P}}=70,93(65,50 ; 76,37)$ & $\widehat{S_{P}}=63,54(49,11 ; 77,04)$ \\
\hline (c) $\lambda_{11}=\lambda_{00}=80 \%$ & Positivo & 652 & 92 & 186 & $\widehat{S_{E}}=79,71(76,77 ; 82,64)$ & $\widehat{S_{E}}=77,23(69,82 ; 83,66)$ \\
\hline$\lambda_{01}=\lambda_{10}=70 \%$ & Negativo & 146 & 262 & 127 & $\widehat{S_{P}}=76,97(72,08 ; 81,87)$ & $\widehat{S_{P}}=67,85(50,29 ; 83,38)$ \\
\hline (e) $\lambda_{11}=\lambda_{00}=80 \%$ & Positivo & 652 & 69 & 207 & $\widehat{S_{E}}=82,92(80,11 ; 85,74)$ & $\widehat{S_{E}}=79,66(71,28 ; 87,47)$ \\
\hline$\lambda_{01}=\lambda_{10}=60 \%$ & Negativo & 125 & 262 & 148 & $\widehat{S_{P}}=80,31(75,76 ; 84,86)$ & $\widehat{S_{P}}=69,24(50,65 ; 85,96)$ \\
\hline (f) $\lambda_{11}=\lambda_{00}=80 \%$ & Positivo & 652 & 58 & 220 & $\widehat{S_{E}}=84,89(82,17 ; 87,61)$ & $\widehat{S_{E}}=81,20(71,30 ; 89,48)$ \\
\hline (h) $\lambda_{11}=\lambda_{00}=80 \%$ & Positivo & 652 & 34 & 232 & $\widehat{S_{E}}=89,62(87,18 ; 92,06)$ & $\widehat{S_{E}}=83,69(71,60 ; 93,67)$ \\
\hline$\lambda_{01}=\lambda_{10}=30 \%$ & Negativo & 62 & 262 & 190 & $\widehat{S_{P}}=90,37(87,18 ; 93,56)$ & $\widehat{S_{P}}=76,12(52,10 ; 93,38)$ \\
\hline (i) $\lambda_{11}=\lambda_{00}=80 \%$ & Positivo & 652 & 23 & 255 & $\widehat{S_{E}}=92,39(90,20 ; 94,59)$ & $\widehat{S_{E}}=85,52(72,75 ; 95,62)$ \\
\hline$\lambda_{01}=\lambda_{10}=20 \%$ & Negativo & 42 & 262 & 231 & $\widehat{S_{P}}=93,57(90,97 ; 96,17)$ & $\widetilde{S_{P}}=79,08(53,76 ; 95,19)$ \\
\hline (j) $\lambda_{11}=\lambda_{00}=80 \%$ & Positivo & 652 & 11 & 267 & $\widehat{S_{E}}=95,84(94,11 ; 97,57)$ & $\widehat{S_{E}}=87,97(72,40 ; 97,96)$ \\
\hline$\lambda_{01}=\lambda_{10}=10 \%$ & Negativo & 21 & 262 & 252 & $\widehat{S_{P}}=96,98(95,20 ; 98,75)$ & $\widehat{S_{P}}=82,75(52,27 ; 97,93)$ \\
\hline
\end{tabular}


Tabela 39: Resultados das simulações para cada porcentagem de verificação - dados de WEINER et al. (1979) - distribuições a priori não informativas (diferentes probabilidades de verificação).

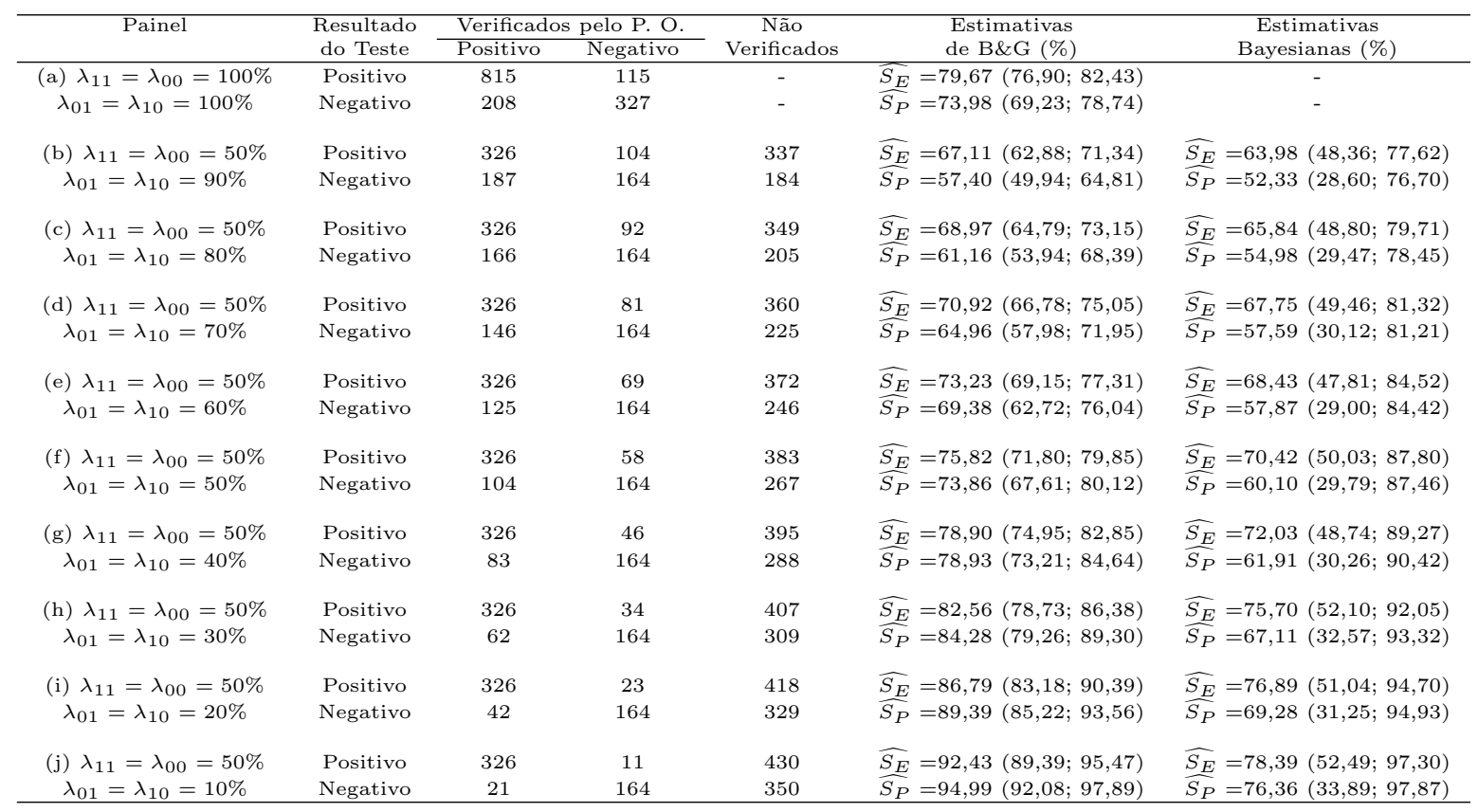

Tabela 40: Resultados das simulações para cada porcentagem de verificação - dados de WEINER et al. (1979) - distribuições a priori não informativas (diferentes probabilidades de verificação).

\begin{tabular}{|c|c|c|c|c|c|c|}
\hline Painel & $\begin{array}{l}\text { Resultado } \\
\text { do Teste }\end{array}$ & $\begin{array}{l}\text { Verificado } \\
\text { Positivo } \\
\end{array}$ & $\frac{\text { pelo P. O. }}{\text { Negativo }}$ & $\begin{array}{c}\text { Não } \\
\text { Verificados }\end{array}$ & $\begin{array}{c}\text { Estimativas } \\
\text { de B\&G }(\%)\end{array}$ & $\begin{array}{c}\text { Estimativas } \\
\text { Bayesianas (\%) }\end{array}$ \\
\hline$\lambda_{01}=\lambda_{10}=100 \%$ & Negativo & 208 & 327 & - & $\widehat{S_{P}}=73,98(69,23 ; 78,74)$ & - \\
\hline$\lambda_{01}=\lambda_{10}=10 \%$ & Negativo & 21 & 294 & 220 & $\widehat{S_{P}}=97,32(95,75 ; 98,90)$ & $\widehat{S_{P}}=86,51(64,04 ; 98,20)$ \\
\hline (c) $\lambda_{11}=\lambda_{00}=80 \%$ & Positivo & 652 & 23 & 255 & $\widehat{S_{E}}=92,39(90,20 ; 94,59)$ & $\widehat{S_{E}}=85,46(71,76 ; 95,64)$ \\
\hline$\lambda_{01}=\lambda_{10}=30 \%$ & Negativo & 62 & 229 & 244 & $\widehat{S_{P}}=88,69(85,02 ; 92,35)$ & $\widehat{S_{P}}=68,65(40,14 ; 92,86)$ \\
\hline (e) $\lambda_{11}=\lambda_{00}=60 \%$ & Positivo & 489 & 46 & 395 & $\widehat{S_{E}}=84,22(81,17 ; 87,28)$ & $\widehat{S_{E}}=77,36(61,34 ; 91,20)$ \\
\hline$\lambda_{01}=\lambda_{10}=40 \%$ & Negativo & 83 & 196 & 256 & $\widehat{S_{P}}=82,46(77,59 ; 87,33)$ & $\widehat{S_{P}}=62,56(33,43 ; 89,67)$ \\
\hline (f) $\lambda_{11}=\lambda_{00}=50 \%$ & Positivo & 408 & 58 & 464 & $\widehat{S_{E}}=79,68(76,29 ; 83,08)$ & $\widehat{S_{E}}=73,63(53,39 ; 89,18)$ \\
\hline (h) $\lambda_{11}=\lambda_{00}=30 \%$ & Positivo & 245 & 81 & 604 & $\widehat{S_{E}}=67,62(63,44 ; 71,79)$ & $\widehat{S_{E}}=62,94(38,47 ; 83,56)$ \\
\hline$\lambda_{01}=\lambda_{10}=70 \%$ & Negativo & 146 & 98 & 291 & $\widehat{S_{P}}=47,64(38,02 ; 57,27)$ & $\widehat{S_{P}}=43,76(14,58 ; 81,08)$ \\
\hline (i) $\lambda_{11}=\lambda_{00}=20 \%$ & Positivo & 163 & 92 & 675 & $\widehat{S_{E}}=60,73(55,76 ; 65,69)$ & $\widehat{S_{E}}=56,35(26,64 ; 82,10)$ \\
\hline$\lambda_{01}=\lambda_{10}=80 \%$ & Negativo & 166 & 65 & 304 & $\widehat{S_{P}}=30,97(20,07 ; 41,87)$ & $\widetilde{S_{P}}=35,12(8,07 ; 77,41)$ \\
\hline (j) $\lambda_{11}=\lambda_{00}=10 \%$ & Positivo & 82 & 104 & 744 & $\widehat{S_{E}}=47,41(40,12 ; 54,71)$ & $\widehat{S_{E}}=47,50(14,88 ; 80,26)$ \\
\hline$\lambda_{01}=\lambda_{10}=90 \%$ & Negativo & 187 & 33 & 315 & $\widehat{S_{P}}=13,37(2,10 ; 24,64)$ & $\widehat{S_{P}}=29,03(3,44 ; 73,84)$ \\
\hline
\end{tabular}


Tabela 41: Resultados das simulações para cada porcentagem de verificação - dados de HIGGINS e KELLER (1973) - distribuições a priori não informativas (diferentes probabilidades de verificação).

\begin{tabular}{|c|c|c|c|c|c|c|}
\hline Painel & $\begin{array}{l}\text { Resultado } \\
\text { do Teste }\end{array}$ & $\begin{array}{l}\text { Verificads } \\
\text { Positivo } \\
\end{array}$ & Verificados pelo P. O. & $\begin{array}{c}\text { Não } \\
\text { Verificados }\end{array}$ & $\begin{array}{r}\text { Estimativas } \\
\text { de B\&G }(\%)\end{array}$ & $\begin{array}{c}\text { Estimativas } \\
\text { Bayesianas (\%) }\end{array}$ \\
\hline$\lambda_{01}=\lambda_{10}=100 \%$ & Negativo & 256 & 1409 & - & $\widehat{S_{P}}=91,32(89,85 ; 92,79)$ & - \\
\hline$\lambda_{01}=\lambda_{10}=90 \%$ & Negativo & 230 & 1409 & 26 & $\widehat{S_{P}}=91,71(90,26 ; 93,15)$ & $\widehat{S_{P}}=91,75(90,17 ; 93,15)$ \\
\hline (c) $\lambda_{11}=\lambda_{00}=100 \%$ & Positivo & 65 & 107 & 27 & $\widehat{S_{E}}=26,23(15,90 ; 36,56)$ & $\widehat{S_{E}}=25,37(18,85 ; 32,75)$ \\
\hline$\lambda_{01}=\lambda_{10}=70 \%$ & Negativo & 179 & 1409 & 77 & $\widehat{S_{P}}=92,62(91,22 ; 94,02)$ & $\widehat{S_{P}}=92,64(90,56 ; 94,66)$ \\
\hline (e) $\lambda_{11}=\lambda_{00}=100 \%$ & Positivo & 65 & 80 & 54 & $\widehat{S_{E}}=35,22(24,58 ; 45,87)$ & $\widehat{S_{E}}=31,25(19,94 ; 44,29)$ \\
\hline$\lambda_{01}=\lambda_{10}=60 \%$ & Negativo & 154 & 1409 & 102 & $\widehat{S_{P}}=93,18(91,81 ; 94,55)$ & $\widehat{S_{P}}=93,12(90,58 ; 95,32)$ \\
\hline (f) $\lambda_{11}=\lambda_{00}=100 \%$ & Positivo & 65 & 67 & 67 & $\widehat{S_{E}}=41,41(30,80 ; 52,01)$ & $\widehat{S_{E}}=34,31(20,01 ; 50,52)$ \\
\hline (h) $\lambda_{11}=\lambda_{00}=100 \%$ & Positivo & 65 & 40 & 94 & $\widehat{S_{E}}=58,81(49,26 ; 68,36)$ & $\widehat{S_{E}}=45,67(22,98 ; 67,17)$ \\
\hline$\lambda_{01}=\lambda_{10}=30 \%$ & Negativo & 77 & 1409 & 179 & $\widehat{S_{P}}=95,41 \quad(94,14 ; 96,69)$ & $\widetilde{S_{P}}=94,72(91,34 ; 97,73)$ \\
\hline (i) $\lambda_{11}=\lambda_{00}=100 \%$ & Positivo & 65 & 27 & 107 & $\widehat{S_{E}}=70,74(62,51 ; 78,96)$ & $\widehat{S_{E}}=50,89(22,18 ; 76,97)$ \\
\hline$\lambda_{01}=\lambda_{10}=20 \%$ & Negativo & 51 & 1409 & 205 & $\widetilde{S_{P}}=96,49(95,29 ; 97,70)$ & $\widehat{S_{P}}=94,89(91,35 ; 98,41)$ \\
\hline (j) $\lambda_{11}=\lambda_{00}=100 \%$ & Positivo & 65 & 13 & 121 & $\widehat{S_{E}}=84,61(78,66 ; 90,56)$ & $\widehat{S_{E}}=61,50(25,65 ; 88,34)$ \\
\hline$\lambda_{01}=\lambda_{10}=10 \%$ & Negativo & 26 & 1409 & 230 & $\widehat{S_{P}}=98,01 \quad(96,99 ; 99,03)$ & $\widehat{S_{P}}=95,87(91,55 ; 99,39)$ \\
\hline
\end{tabular}

Tabela 42: Resultados das simulações para cada porcentagem de verificação - dados de HIGGINS e KELLER (1973) - distribuições a priori não informativas (diferentes probabilidades de verificação).

\begin{tabular}{|c|c|c|c|c|c|c|}
\hline Painel & $\begin{array}{l}\text { Resultado } \\
\text { do Teste }\end{array}$ & \multicolumn{2}{|c|}{ Verificados pelo P. O. } & $\begin{array}{c}\text { Não } \\
\text { Verificados }\end{array}$ & $\begin{array}{c}\text { Estimativas } \\
\text { de B\&G }(\%)\end{array}$ & $\begin{array}{c}\text { Estimativas } \\
\text { Bayesianas (\%) }\end{array}$ \\
\hline$\lambda_{01}=\lambda_{10}=100 \%$ & Negativo & 256 & 1409 & - & $\widehat{S_{P}}=91,32(89,85 ; 92,79)$ & - \\
\hline$\lambda_{01}=\lambda_{10}=90 \%$ & Negativo & 230 & 1127 & 308 & $\widehat{S_{P}}=90,86(89,30 ; 92,41)$ & $\widehat{S_{P}}=90,64(88,18 ; 92,79)$ \\
\hline (c) $\lambda_{11}=\lambda_{00}=80 \%$ & Positivo & 52 & 107 & 40 & $\widehat{S_{E}}=20,25(9,65 ; 30,85)$ & $\widehat{S_{E}}=18,06(8,71 ; 31,39)$ \\
\hline$\lambda_{01}=\lambda_{10}=70 \%$ & Negativo & 179 & 1127 & 359 & $\widehat{S_{P}}=91,81 \quad(90,31 ; 93,31)$ & $\widehat{S_{P}}=91,56(88,36 ; 94,32)$ \\
\hline (e) $\lambda_{11}=\lambda_{00}=80 \%$ & Positivo & 52 & 80 & 67 & $\widehat{S_{E}}=28,14(16,81 ; 39,47)$ & $\widehat{S_{E}}=23,93(9,44 ; 42,66)$ \\
\hline$\lambda_{01}=\lambda_{10}=60 \%$ & Negativo & 154 & 1127 & 384 & $\widehat{S_{P}}=92,39(90,91 ; 93,87)$ & $\widehat{S_{P}}=92,14(88,73 ; 95,19)$ \\
\hline (f) $\lambda_{11}=\lambda_{00}=80 \%$ & Positivo & 52 & 67 & 80 & $\widehat{S_{E}}=33,87(22,31 ; 45,42)$ & $\widehat{S_{E}}=27,30(8,74 ; 50,22)$ \\
\hline (h) $\lambda_{11}=\lambda_{00}=80 \%$ & Positivo & 52 & 40 & 107 & $\widehat{S_{E}}=51,36(40,32 ; 62,42)$ & $\widehat{S_{E}}=36,73(10,06 ; 66,14)$ \\
\hline$\lambda_{01}=\lambda_{10}=30 \%$ & Negativo & 77 & 1127 & 461 & $\widehat{S_{P}}=94,74(93,32 ; 96,15)$ & $\widehat{S_{P}}=93,84(88,86 ; 97,61)$ \\
\hline (i) $\lambda_{11}=\lambda_{00}=80 \%$ & Positivo & 52 & 27 & 120 & $\widehat{S_{E}}=64,50(54,67 ; 74,33)$ & $\widehat{S_{E}}=41,53(11,43 ; 75,79)$ \\
\hline$\lambda_{01}=\lambda_{10}=20 \%$ & Negativo & 51 & 1127 & 487 & $\widehat{S_{P}}=95,91(94,54 ; 97,27)$ & $\widehat{S_{P}}=94,49(89,46 ; 98,41)$ \\
\hline (j) $\lambda_{11}=\lambda_{00}=80 \%$ & Positivo & 52 & 13 & 134 & $\widehat{S_{E}}=80,92(73,62 ; 88,22)$ & $\widehat{S_{E}}=55,25(12,71 ; 88,20)$ \\
\hline$\lambda_{01}=\lambda_{10}=10 \%$ & Negativo & 26 & 1127 & 512 & $\widehat{S_{P}}=97,61(96,41 ; 98,81)$ & $\widehat{S_{P}}=95,61(90,05 ; 99,32)$ \\
\hline
\end{tabular}


Tabela 43: Resultados das simulações para cada porcentagem de verificação - dados de HIGGINS e KELLER (1973) - distribuições a priori não informativas (diferentes probabilidades de verificação).

\begin{tabular}{|c|c|c|c|c|c|c|}
\hline Painel & $\begin{array}{l}\text { Resultado } \\
\text { do Teste }\end{array}$ & $\begin{array}{l}\text { Verificado } \\
\text { Positivo } \\
\end{array}$ & Verificados pelo P. O. & $\begin{array}{c}\text { Não } \\
\text { Verificados }\end{array}$ & $\begin{array}{c}\text { Estimativas } \\
\text { de B\&G }(\%)\end{array}$ & $\begin{array}{c}\text { Estimativas } \\
\text { Bayesianas (\%) }\end{array}$ \\
\hline$\lambda_{01}=\lambda_{10}=100 \%$ & Negativo & 256 & 1409 & - & $\widehat{S_{P}}=91,32(89,85 ; 92,79)$ & - \\
\hline$\lambda_{01}=\lambda_{10}=90 \%$ & Negativo & 230 & 705 & 730 & $\widehat{S_{P}}=88,92(87,12 ; 90,73)$ & $\widehat{S_{P}}=87,71(81,09 ; 92,18)$ \\
\hline (c) $\lambda_{11}=\lambda_{00}=50 \%$ & Positivo & 33 & 107 & 59 & $\widehat{S_{E}}=11,11(0,06 ; 21,60)$ & $\widehat{S_{E}}=12,20(3,40 ; 28,99)$ \\
\hline$\lambda_{01}=\lambda_{10}=70 \%$ & Negativo & 179 & 705 & 781 & $\widehat{S_{P}}=90,02(88,29 ; 91,74)$ & $\widehat{S_{P}}=88,62(81,05 ; 93,87)$ \\
\hline (e) $\lambda_{11}=\lambda_{00}=50 \%$ & Positivo & 33 & 88 & 88 & $\widehat{S_{E}}=16,43(4,49 ; 28,38)$ & $\widehat{S_{E}}=17,88(3,58 ; 41,65)$ \\
\hline$\lambda_{01}=\lambda_{10}=60 \%$ & Negativo & 154 & 705 & 806 & $\widehat{S_{P}}=90,57(88,86 ; 92,28)$ & $\widehat{S_{P}}=89,98(81,22 ; 94,88)$ \\
\hline (f) $\lambda_{11}=\lambda_{00}=50 \%$ & Positivo & 33 & 67 & 99 & $\widehat{S_{E}}=20,42(7,71 ; 33,14)$ & $\widehat{S_{E}}=20,84(3,89 ; 48,20)$ \\
\hline (h) $\lambda_{11}=\lambda_{00}=50 \%$ & Positivo & 33 & 40 & 126 & $\widehat{S_{E}}=35,43(21,55 ; 49,31)$ & $\widehat{S_{E}}=23,37(3,85 ; 63,31)$ \\
\hline$\lambda_{01}=\lambda_{10}=30 \%$ & Negativo & 77 & 705 & 883 & $\widehat{S_{P}}=93,23(91,55 ; 94,90)$ & $\widehat{S_{P}}=91,22(81,47 ; 97,30)$ \\
\hline (i) $\lambda_{11}=\lambda_{00}=50 \%$ & Positivo & 33 & 27 & 139 & $\widehat{S_{E}}=49,35(35,83 ; 62,87)$ & $\widehat{S_{E}}=36,54(4,93 ; 76,94)$ \\
\hline$\lambda_{01}=\lambda_{10}=20 \%$ & Negativo & 51 & 705 & 909 & $\widehat{S_{P}}=94,55(92,86 ; 96,23)$ & $\widehat{S_{P}}=92,77(82,75 ; 98,46)$ \\
\hline (j) $\lambda_{11}=\lambda_{00}=50 \%$ & Positivo & 33 & 13 & 153 & $\widehat{S_{E}}=70,68(59,74 ; 81,62)$ & $\widehat{S_{E}}=47,60(6,54 ; 87,02)$ \\
\hline$\lambda_{01}=\lambda_{10}=10 \%$ & Negativo & 26 & 705 & 934 & $\widehat{S_{P}}=96,62(95,01 ; 98,22)$ & $\widehat{S_{P}}=94,15(86,20 ; 99,24)$ \\
\hline
\end{tabular}

Tabela 44: Resultados das simulações para cada porcentagem de verificação - dados de HIGGINS e KELLER (1973) - distribuições a priori não informativas (diferentes probabilidades de verificação).

\begin{tabular}{|c|c|c|c|c|c|c|}
\hline Painel & $\begin{array}{c}\text { Resultado } \\
\text { do Teste }\end{array}$ & \multicolumn{2}{|c|}{ Verificados pelo P. O. } & $\begin{array}{c}\text { Não } \\
\text { Verificados }\end{array}$ & $\begin{array}{c}\text { Estimativas } \\
\text { de B\&G (\%) }\end{array}$ & $\begin{array}{c}\text { Estimativas } \\
\text { Bayesianas (\%) }\end{array}$ \\
\hline$\lambda_{01}=\lambda_{10}=100 \%$ & Negativo & 256 & 1409 & - & $\widehat{S_{P}}=91,32(89,85 ; 92,79)$ & - \\
\hline$\lambda_{01}=\lambda_{10}=10 \%$ & Negativo & 26 & 1268 & 371 & $\widehat{S_{P}}=97,82(96,71 ; 98,92)$ & $\widehat{S_{P}}=95,27(90,87 ; 99,31)$ \\
\hline (c) $\lambda_{11}=\lambda_{00}=80 \%$ & Positivo & 52 & 27 & 120 & $\widehat{S_{E}}=64,50(54,67 ; 74,33)$ & $\widehat{S_{E}}=43,90(11,10 ; 77,42)$ \\
\hline$\lambda_{01}=\lambda_{10}=30 \%$ & Negativo & 77 & 986 & 602 & $\widehat{S_{P}}=94,35(92,86 ; 95,83)$ & $\widehat{S_{P}}=93,43(87,78 ; 97,58)$ \\
\hline (e) $\lambda_{11}=\lambda_{00}=60 \%$ & Positivo & 39 & 54 & 106 & $\widehat{S_{E}}=31,75(18,86 ; 44,65)$ & $\widehat{S_{E}}=25,84(5,54 ; 57,33)$ \\
\hline$\lambda_{01}=\lambda_{10}=40 \%$ & Negativo & 102 & 845 & 718 & $\widehat{S_{P}}=92,78(91,20 ; 94,37)$ & $\widehat{S_{P}}=92,03(84,54 ; 96,56)$ \\
\hline (f) $\lambda_{11}=\lambda_{00}=50 \%$ & Positivo & 33 & 67 & 99 & $\widehat{S_{E}}=20,43(7,71 ; 33,14)$ & $\widehat{S_{E}}=19,67(4,25 ; 47,47)$ \\
\hline (h) $\lambda_{11}=\lambda_{00}=30 \%$ & Positivo & 20 & 94 & 85 & $\widehat{S_{E}}=6,58(0,03 ; 13,18)$ & $\widehat{S_{E}}=13,45(1,98 ; 35,22)$ \\
\hline$\lambda_{01}=\lambda_{10}=70 \%$ & Negativo & 179 & 423 & 1063 & $\widehat{S_{P}}=87,70(85,66 ; 89,74)$ & $\widehat{S_{P}}=87,97(75,21 ; 93,98)$ \\
\hline (i) $\lambda_{11}=\lambda_{00}=20 \%$ & Positivo & 13 & 107 & 79 & $\widehat{S_{E}}=2,98(0,01 ; 6,05)$ & $\widehat{S_{E}}=6,93(0,08 ; 25,18)$ \\
\hline$\lambda_{01}=\lambda_{10}=80 \%$ & Negativo & 205 & 282 & 1178 & $\widehat{S_{P}}=84,45(81,93 ; 86,98)$ & $\widehat{S_{P}}=79,61 \quad(59,66 ; 92,43)$ \\
\hline (j) $\lambda_{11}=\lambda_{00}=10 \%$ & Positivo & 7 & 121 & 71 & $\widehat{S_{E}}=1,04(0,01 ; 3,16)$ & $\widehat{S_{E}}=5,18(0,05 ; 20,89)$ \\
\hline$\lambda_{01}=\lambda_{10}=90 \%$ & Negativo & 230 & 141 & 1294 & $\widehat{S_{P}}=77,08(73,05 ; 81,12)$ & $\widehat{S_{P}}=74,29(44,49 ; 91,46)$ \\
\hline
\end{tabular}


Tabela 45: Resultados das simulações para cada porcentagem de verificação - dados de BEGG e MCNEIL (1988) - distribuições a priori não informativas (diferentes probabilidades de verificação).

\begin{tabular}{|c|c|c|c|c|c|c|}
\hline Painel & $\begin{array}{l}\text { Resultado } \\
\text { do Teste }\end{array}$ & \multicolumn{2}{|c|}{ Verificados pelo P. O. } & $\begin{array}{c}\text { Não } \\
\text { Verificados } \\
\end{array}$ & $\begin{array}{r}\text { Estimativas } \\
\text { de B\&G }(\%) \\
\end{array}$ & $\begin{array}{c}\text { Estimativas } \\
\text { Bayesianas (\%) }\end{array}$ \\
\hline$\lambda_{01}=\lambda_{10}=100 \%$ & Negativo & 179 & 372 & - & $\widehat{S_{P}}=82,30(78,42 ; 86,17)$ & - \\
\hline$\lambda_{01}=\lambda_{10}=90 \%$ & Negativo & 161 & 372 & 18 & $\widehat{S_{P}}=83,95(80,25 ; 87,65)$ & $\widehat{S_{P}}=83,28(79,34 ; 86,50)$ \\
\hline (c) $\lambda_{11}=\lambda_{00}=100 \%$ & Positivo & 302 & 64 & 16 & $\widehat{S_{E}}=67,32(62,05 ; 72,59)$ & $\widehat{S_{E}}=66,06(60,80 ; 71,42)$ \\
\hline$\lambda_{01}=\lambda_{10}=70 \%$ & Negativo & 125 & 372 & 54 & $\widehat{S_{P}}=87,34(84,05 ; 90,64)$ & $\widehat{S_{P}}=85,63(80,68 ; 89,93)$ \\
\hline (e) $\lambda_{11}=\lambda_{00}=100 \%$ & Positivo & 302 & 48 & 32 & $\widehat{S_{E}}=72,81(67,80 ; 77,82)$ & $\widehat{S_{E}}=69,63(61,47 ; 77,06)$ \\
\hline$\lambda_{01}=\lambda_{10}=60 \%$ & Negativo & 107 & 372 & 72 & $\widehat{S_{P}}=89,09(86,03 ; 92,15)$ & $\widehat{S_{P}}=86,75(82,20 ; 91,36)$ \\
\hline (f) $\lambda_{11}=\lambda_{00}=100 \%$ & Positivo & 302 & 40 & 40 & $\widehat{S_{E}}=75,86(71,03 ; 80,70)$ & $\widehat{S_{E}}=71,22(62,16 ; 80,24)$ \\
\hline (h) $\lambda_{11}=\lambda_{00}=100 \%$ & Positivo & 302 & 24 & 56 & $\widehat{S_{E}}=83,52(79,25 ; 87,79)$ & $\widehat{S_{E}}=75,82(61,69 ; 88,17)$ \\
\hline$\lambda_{01}=\lambda_{10}=30 \%$ & Negativo & 54 & 372 & 125 & $\widehat{S_{P}}=94,48(92,26 ; 96,69)$ & $\widehat{S_{P}}=90,24(82,51 ; 95,82)$ \\
\hline (i) $\lambda_{11}=\lambda_{00}=100 \%$ & Positivo & 302 & 16 & 64 & $\widehat{S_{E}}=88,18(84,42 ; 91,95)$ & $\widehat{S_{E}}=79,53(64,24 ; 92,26)$ \\
\hline$\lambda_{01}=\lambda_{10}=20 \%$ & Negativo & 36 & 372 & 143 & $\widehat{S_{P}}=96,32(94,52 ; 98,11)$ & $\widehat{S_{P}}=91,34(82,37 ; 97,32)$ \\
\hline (j) $\lambda_{11}=\lambda_{00}=100 \%$ & Positivo & 302 & 8 & 72 & $\widehat{S_{E}}=93,60(90,69 ; 96,51)$ & $\widehat{S_{E}}=82,33(64,33 ; 95,82)$ \\
\hline$\lambda_{01}=\lambda_{10}=10 \%$ & Negativo & 18 & 372 & 161 & $\widehat{S_{P}}=98,16(96,89 ; 99,43)$ & $\widehat{S_{P}}=93,19(83,39 ; 98,80)$ \\
\hline
\end{tabular}

Tabela 46: Resultados das simulações para cada porcentagem de verificação - dados de BEGG e MCNEIL (1988) - distribuições a priori não informativas (diferentes probabilidades de verificação).

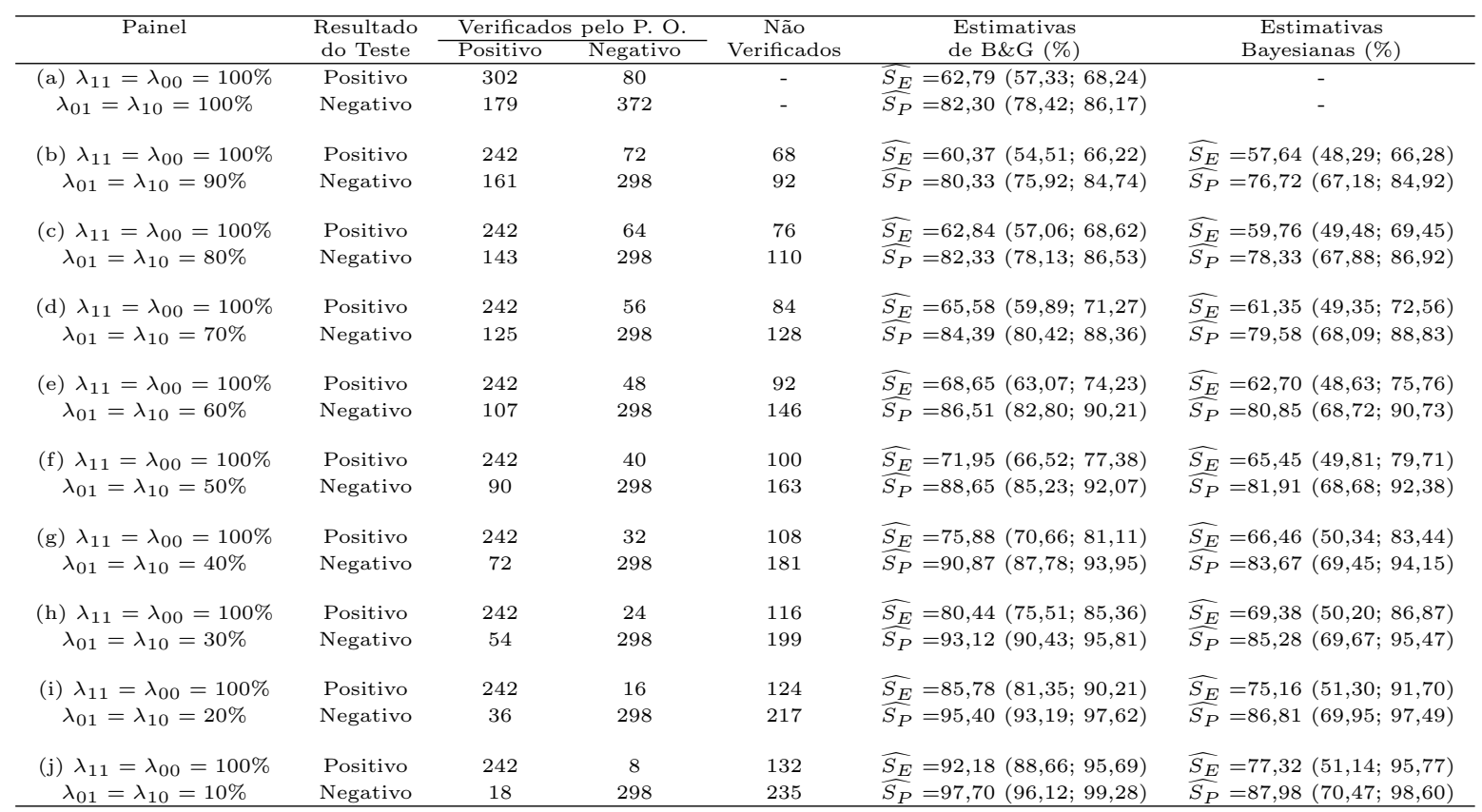


Tabela 47: Resultados das simulações para cada porcentagem de verificação - dados de BEGG e MCNEIL (1988) - distribuições a priori não informativas (diferentes probabilidades de verificação).

\begin{tabular}{|c|c|c|c|c|c|c|}
\hline Painel & $\begin{array}{c}\text { Resultado } \\
\text { do Teste }\end{array}$ & \multicolumn{2}{|c|}{ Verificados pelo P. O. } & $\begin{array}{c}\text { Não } \\
\text { Verificados }\end{array}$ & $\begin{array}{c}\text { Estimativas } \\
\text { de B\&G }(\%)\end{array}$ & $\begin{array}{c}\text { Estimativas } \\
\text { Bayesianas (\%) }\end{array}$ \\
\hline$\lambda_{01}=\lambda_{10}=100 \%$ & Negativo & 179 & 372 & - & $\widehat{S_{P}}=82,30(78,42 ; 86,17)$ & - \\
\hline$\lambda_{01}=\lambda_{10}=90 \%$ & Negativo & 161 & 186 & 204 & $\widehat{S_{P}}=70,54(63,34 ; 76,74)$ & $\widehat{S_{P}}=65,94(45,21 ; 84,11)$ \\
\hline (c) $\lambda_{11}=\lambda_{00}=100 \%$ & Positivo & 151 & 64 & 167 & $\widehat{S_{E}}=52,83(46,00 ; 59,67)$ & $\widehat{S_{E}}=50,19(31,03 ; 69,31)$ \\
\hline$\lambda_{01}=\lambda_{10}=70 \%$ & Negativo & 125 & 186 & 240 & $\widehat{S_{P}}=76,13(70,41 ; 81,85)$ & $\widehat{S_{P}}=70,42(46,68 ; 87,87)$ \\
\hline (e) $\lambda_{11}=\lambda_{00}=100 \%$ & Positivo & 151 & 48 & 183 & $\widehat{S_{E}}=62,27(55,81 ; 68,72)$ & $\widehat{S_{E}}=57,21(35,79 ; 74,73)$ \\
\hline$\lambda_{01}=\lambda_{10}=60 \%$ & Negativo & 107 & 186 & 188 & $\widehat{S_{P}}=76,82(70,85 ; 82,79)$ & $\widehat{S_{P}}=69,55(45,80 ; 87,95)$ \\
\hline (f) $\lambda_{11}=\lambda_{00}=100 \%$ & Positivo & 151 & 40 & 191 & $\widehat{S_{E}}=62,27(55,81 ; 68,72)$ & $\widehat{S_{E}}=55,07(36,41 ; 74,47)$ \\
\hline (h) $\lambda_{11}=\lambda_{00}=100 \%$ & Positivo & 151 & 24 & 207 & $\widehat{S_{E}}=72,67(66,25 ; 79,08)$ & $\widehat{S_{E}}=61,93(32,30 ; 87,17)$ \\
\hline$\lambda_{01}=\lambda_{10}=30 \%$ & Negativo & 54 & 186 & 311 & $\widehat{S_{P}}=89,07(84,95 ; 93,19)$ & $\widehat{S_{P}}=77,17(48,21 ; 95,54)$ \\
\hline (i) $\lambda_{11}=\lambda_{00}=100 \%$ & Positivo & 151 & 16 & 215 & $\widehat{S_{E}}=79,45(73,40 ; 85,49)$ & $\widehat{S_{E}}=63,46(32,06 ; 90,21)$ \\
\hline$\lambda_{01}=\lambda_{10}=20 \%$ & Negativo & 36 & 186 & 329 & $\widehat{S_{P}}=92,65(89,21 ; 96,10)$ & $\widehat{S_{P}}=78,26(47,63 ; 97,14)$ \\
\hline (j) $\lambda_{11}=\lambda_{00}=100 \%$ & Positivo & 151 & 8 & 223 & $\widehat{S_{E}}=88,18(83,06 ; 93,30)$ & $\widehat{S_{E}}=71,56(39,09 ; 95,80)$ \\
\hline$\lambda_{01}=\lambda_{10}=10 \%$ & Negativo & 18 & 186 & 347 & $\widehat{S_{P}}=96,32(93,82 ; 98,81)$ & $\widehat{S_{P}}=84,97(55,24 ; 98,59)$ \\
\hline
\end{tabular}

Tabela 48: Resultados das simulações para cada porcentagem de verificação - dados de BEGG e MCNEIL (1988) - distribuições a priori não informativas (diferentes probabilidades de verificação).

\begin{tabular}{|c|c|c|c|c|c|c|}
\hline Painel & $\begin{array}{l}\text { Resultado } \\
\text { do Teste }\end{array}$ & \multicolumn{2}{|c|}{ Verificados pelo P. O. } & $\begin{array}{c}\text { Não } \\
\text { Verificados }\end{array}$ & $\begin{array}{c}\text { Estimativas } \\
\text { de B\&G (\%) }\end{array}$ & $\begin{array}{c}\text { Estimativas } \\
\text { Bayesianas (\%) }\end{array}$ \\
\hline$\lambda_{01}=\lambda_{10}=100 \%$ & Negativo & 179 & 372 & - & $\widehat{S_{P}}=82,30(78,42 ; 86,17)$ & - \\
\hline$\lambda_{01}=\lambda_{10}=10 \%$ & Negativo & 18 & 335 & 198 & $\widehat{S_{P}}=97,96(96,55 ; 99,36)$ & $\widehat{S_{P}}=91,70(78,17 ; 98,68)$ \\
\hline (c) $\lambda_{11}=\lambda_{00}=80 \%$ & Positivo & 242 & 16 & 124 & $\widehat{S_{E}}=85,78(81,35 ; 90,21)$ & $\widehat{S_{E}}=73,36(51,68 ; 91,40)$ \\
\hline$\lambda_{01}=\lambda_{10}=30 \%$ & Negativo & 54 & 260 & 237 & $\widehat{S_{P}}=92,12(89,07 ; 95,18)$ & $\widehat{S_{P}}=81,69(62,17 ; 95,51)$ \\
\hline (e) $\lambda_{11}=\lambda_{00}=60 \%$ & Positivo & 181 & 32 & 169 & $\widehat{S_{E}}=70,71(64,65 ; 76,77)$ & $\widehat{S_{E}}=62,92(37,15 ; 83,20)$ \\
\hline$\lambda_{01}=\lambda_{10}=40 \%$ & Negativo & 72 & 223 & 256 & $\widehat{S_{P}}=87,89(83,90 ; 91,88)$ & $\widehat{S_{P}}=78,75(54,98 ; 93,83)$ \\
\hline (f) $\lambda_{11}=\lambda_{00}=50 \%$ & Positivo & 151 & 40 & 191 & $\widehat{S_{E}}=62,70(56,02 ; 69,38)$ & $\widehat{S_{E}}=54,15(30,15 ; 77,87)$ \\
\hline (h) $\lambda_{11}=\lambda_{00}=30 \%$ & Positivo & 91 & 56 & 235 & $\widehat{S_{E}}=44,86(36,80 ; 52,92)$ & $\widehat{S_{E}}=46,13(20,15 ; 72,66)$ \\
\hline$\lambda_{01}=\lambda_{10}=70 \%$ & Negativo & 125 & 112 & 314 & $\widehat{S_{P}}=64,15(56,15 ; 72,15)$ & $\widehat{S_{P}}=64,15(33,37 ; 88,30)$ \\
\hline (i) $\lambda_{11}=\lambda_{00}=20 \%$ & Positivo & 60 & 64 & 258 & $\widehat{S_{E}}=33,73(24,37 ; 43,10)$ & $\widehat{S_{E}}=34,49(11,30 ; 66,73)$ \\
\hline$\lambda_{01}=\lambda_{10}=80 \%$ & Negativo & 143 & 74 & 334 & $\widehat{S_{P}}=48,80(38,66 ; 58,93)$ & $\widehat{S_{P}}=50,17(19,63 ; 85,04)$ \\
\hline (j) $\lambda_{11}=\lambda_{00}=10 \%$ & Positivo & 30 & 72 & 280 & $\widehat{S_{E}}=20,05(8,08 ; 32,02)$ & $\widehat{S_{E}}=30,42(5,96 ; 64,01)$ \\
\hline$\lambda_{01}=\lambda_{10}=90 \%$ & Negativo & 161 & 37 & 353 & $\widehat{S_{P}}=27,63(14,61 ; 40,65)$ & $\widehat{S_{P}}=43,81(11,28 ; 82,85)$ \\
\hline
\end{tabular}


Para as simulações, foram escolhidos três exemplos nos quais os valores da sensibilidade e da especificidade dos testes em estudo são bem discrepantes. Isso foi feito para que se pudesse avaliar o efeito do tamanho amostral, na estimação desses parâmetros de interesse, quando o viés de verificação está presente. Assim, foram escolhidas diferentes distribuições a priori não informativas e informativas, retiradas a partir dos próprios bancos de dados.

Nota-se que a metodologia bayesiana não foi capaz de estimar a sensiblidade e a especificidade de maneira que os valores fossem próximos aos valores reais (banco completo). Mesmo com a escolha das distribuições a priori informativas, o método se comportou de forma insatisfatória. Esta metodologia poderia ter um desempenho melhor, talvez, se a escolha das distribuições a priori fossem obtidas a partir de um especialista da área, já que as obtidas a partir da própria amostra não forneceram resultados diferentes daqueles obtidos a partir das distribuições a priori não informativas. 


\section{Avaliação de Dois Testes Diagnós- ticos na Presença do Viés de Veri-} ficação

Planejamentos de duas fases são utilizados com freqüência, principalmente em estudos epidemiológicos (HAND, 1987). Esse tipo de procedimento é constituído de uma primeira fase, em que uma grande amostra é avaliada por um instrumento de rastreamento de alguma característica para que sejam selecionados indivíduos para uma segunda etapa. Pode ser um estágio que exija um investimento maior e envolva técnicas mais invasivas para detectar algum sintoma de interesse. Pode ser, também, uma fase em que se deseja obter maiores informações sobre os participantes do estudo, para então selecionar-se um grupo que será submetido à verificação através de um exame mais detalhado, o padrão ouro (ZHOU, 1998).

Surge, portanto, a necessidade de se comparar a acurácia dos dois testes utilizados no rastreamento, no que diz respeito a discriminarem indivíduos com e sem uma certa doença. Se os resultados desses testes são do tipo "positivo" ou "negativo", a acurácia pode ser avaliada através dos valores de sensibilidade e especificidade de cada um deles. Se todos os indivíduos forem submetidos à verificação esse objetivo poderia ser alcançado através da aplicação de um teste de Mc Nemar (AGRESTI, 2002), já que os resultados dos dois testes são correlacionados. Quando a informação da verificação não está disponível, por alguns motivos já citados anteriormente, o teste de Mc Nemar é aplicado descartando-se os indivíduos não verificados, o que pode levar ao viés de verificação. Métodos para se avaliar a acurácia de um único teste diagnóstico já foram discutidas na Seção 2. Zhou (1998) propôs uma metodologia para que a acurácia de dois testes diagnósticos seja avaliada quando há indivíduos não verificados pelo padrão ouro, admitindo-se que a verificação independe do estado da doença, o que não é observado na prática. Para contornar esse 
problema, será introduzido um modelo bayesiano, que reconhece dependência entre $V$ e $D$, considerando os parâmetros $\lambda_{111}, \lambda_{101}, \lambda_{011}, \lambda_{001}, \lambda_{110}, \lambda_{100}, \lambda_{010}, \lambda_{000}$.

\subsection{Método de Zhou}

Sejam $T_{1}$ e $T_{2}$ variáveis aleatórias representando os resultados dos testes 1 e 2 , respectivamente, onde $T_{k}=1$ denota um resultado positivo para o teste $k$ e $T_{k}=0$ um resultado negativo $(k=1,2)$. Considere $D$ uma variável aleatória representando o verdadeiro estado de um indivíduo, sendo que $D=1$ se o indivíduo é portador da doença e $D=0$ se o indivíduo é saudável. Seja, também, $V$ uma variável aleatória tal que $V=1$ indica que o indivíduo foi verificado pelo padrão ouro e $V=0$ indica que ele não foi verificado.

O modelo proposto por Zhou (1998), afim de se comparar o desempenho de dois testes diagnósticos na presença do viés de verificação, tem como pressuposto a seguinte relação

$$
P\left(V \mid T_{1}, T_{2}, D\right)=P\left(V \mid T_{1}, T_{2}\right),
$$

ou seja, assume-se que a probabilidade de um indivíduo ser verificado pelo padrão ouro depende apenas dos resultados dos testes e não do verdadeiro estado (positivo ou negativo). Esse é o mesmo pressuposto assumido por Begg e Greenes (1983).

Zhou (1998) define as probabilidades condicionais

$$
\psi_{i j}=P\left(D=1 \mid T_{1}=i, T_{2}=j\right)
$$

para $i=0,1$ e $j=0,1$, e as probabilidades

$$
\eta_{i j}=P\left(T_{1}=i, T_{2}=j\right)
$$

para $i=0,1$ e $j=0,1$, em que $\sum_{i=0}^{1} \sum_{j=0}^{1} \eta_{i j}=1$. Sejam os vetores $\boldsymbol{\psi}=\left(\psi_{00}, \psi_{01}, \psi_{10}, \psi_{11}\right)^{\prime}$, $\boldsymbol{\eta}^{\prime}=\left(\eta_{00}, \eta_{01}, \eta_{10}\right)$ e $\eta_{11}=1-\eta_{00}-\eta_{01}-\eta_{10}$. Tem-se que 


$$
\sum_{i=0}^{1} \sum_{j=0}^{1} \psi_{i j} \eta_{i j}=\sum_{i=0}^{1} \sum_{j=0}^{1} P\left(D=1 \mid T_{1}=i, T_{2}=j\right) P\left(T_{1}=i, T_{2}=j\right)=P(D=1)
$$

ou seja, $P(D=1)=\xi$, que é a prevalência da doença.

Tem-se ainda que

$$
\begin{aligned}
P(D & \left.=1, T_{1}=1\right)=P\left(D=1, T_{1}=1, T_{2}=1\right)+P\left(D=1, T_{1}=1, T_{2}=0\right) \\
& =P\left(D=1 \mid T_{1}=1, T_{2}=1\right) P\left(T_{1}=1, T_{2}=1\right)+ \\
+P(D & \left.=1 \mid T_{1}=1, T_{2}=0\right) P\left(T_{1}=1, T_{2}=0\right) \\
& =\psi_{11} \eta_{11}+\psi_{10} \eta_{10}=\sum_{j=1}^{n} \psi_{1 j} \eta_{1 j} .
\end{aligned}
$$

Analogamente,

$$
\begin{aligned}
P(D & \left.=1, T_{2}=1\right)=P\left(D=1, T_{1}=1, T_{2}=1\right)+P\left(D=1, T_{1}=0, T_{2}=1\right) \\
& =P\left(D=1 \mid T_{1}=1, T_{2}=1\right) P\left(T_{1}=1, T_{2}=1\right)+ \\
+P(D & \left.=1 \mid T_{1}=0, T_{2}=1\right) P\left(T_{1}=0, T_{2}=1\right) \\
& =\psi_{11} \eta_{11}+\psi_{01} \eta_{01}=\sum_{i=0}^{1} \psi_{i 1} \eta_{i 1} .
\end{aligned}
$$

Assim, combinando-se as expressões (49) e (50), tem-se que a sensibilidade do teste 1 é dada por

$$
S_{E 1}=P\left(T_{1}=1 \mid D=1\right)=\frac{P\left(T_{1}=1, D=1\right)}{P(D=1)}=\frac{\sum_{j=0}^{1} \psi_{1 j} \eta_{1 j}}{\xi},
$$

e, das expressões (49) e (51), tem-se que a sensibilidade do teste 2 é dada por 


$$
S_{E 2}=P\left(T_{2}=1 \mid D=1\right)=\frac{P\left(T_{2}=1, D=1\right)}{P(D=1)}=\frac{\sum_{i=0}^{1} \psi_{i 1} \eta_{i 1}}{\xi} .
$$

Agora, considerando que

$$
\begin{aligned}
P(D & \left.=0, T_{1}=0\right)=P\left(D=0, T_{1}=0, T_{2}=0\right)+P\left(D=0, T_{1}=0, T_{2}=1\right) \\
& =P\left(D=0 \mid T_{1}=0, T_{2}=0\right) P\left(T_{1}=0, T_{2}=0\right)+P\left(D=0 \mid T_{1}=0, T_{2}=1\right) P\left(T_{1}=0, T_{2}=1\right) \\
& =\left(1-\psi_{00}\right) \eta_{00}+\left(1-\psi_{01}\right) \eta_{01}=\sum_{j=0}^{1}\left(1-\psi_{0 j}\right) \eta_{0 j} .
\end{aligned}
$$

Analogamente,

$$
\begin{aligned}
P(D & \left.=0, T_{2}=0\right)=P\left(D=0, T_{1}=0, T_{2}=0\right)+P\left(D=0, T_{1}=1, T_{2}=0\right) \\
& =P\left(D=0 \mid T_{1}=0, T_{2}=0\right) P\left(T_{1}=0, T_{2}=0\right)+P\left(D=0 \mid T_{1}=1, T_{2}=0\right) P\left(T_{1}=1, T_{2}=0\right) \\
& =\left(1-\psi_{00}\right) \eta_{00}+\left(1-\psi_{10}\right) \eta_{10}=\sum_{i=0}^{1}\left(1-\psi_{i 0}\right) \eta_{i 0} .
\end{aligned}
$$

Assim, as expressões para as especificidades dos testes 1 e 2 são dadas, respectivamente, por

$$
S_{P 1}=P\left(T_{1}=0 \mid D=0\right)=\frac{P\left(T_{1}=0, D=0\right)}{P(D=0)}=\frac{\sum_{j=0}^{1}\left(1-\psi_{0 j}\right) \eta_{0 j}}{1-\xi}
$$

e

$$
S_{P 2}=P\left(T_{2}=0 \mid D=0\right)=\frac{P\left(T_{2}=0, D=0\right)}{P(D=0)}=\frac{\sum_{i=0}^{1}\left(1-\psi_{i 0}\right) \eta_{i 0}}{1-\xi} .
$$

A Tabela 49 sumariza as combinações entre os resultados dos testes 1 e 2, de acordo com o verdadeiro status da doença $(D)$ e o da verificação pelo padrão ouro $(V)$.

Note-se que $n=n_{11}+n_{10}+n_{01}+n_{00}$, que é o tamanho amostral. Admitindo-se o 
Tabela 49: Combinações de resultados dos testes 1 e 2, de acordo com $D$ e $V$.

\begin{tabular}{cccccc}
\hline & \multicolumn{2}{c}{$T_{1}=1$} & & \multicolumn{2}{c}{$T_{1}=0$} \\
\cline { 2 - 3 } \cline { 5 - 6 } & $T_{2}=1$ & $T_{2}=0$ & & $T_{2}=1$ & $T_{2}=0$ \\
\hline$V=1, D=1$ & $s_{11}$ & $s_{10}$ & & $s_{01}$ & $s_{00}$ \\
$V=1, D=0$ & $r_{11}$ & $r_{10}$ & & $r_{01}$ & $r_{00}$ \\
$V=0$ & $u_{11}$ & $u_{10}$ & & $u_{01}$ & $u_{00}$ \\
Total & $n_{11}$ & $n_{10}$ & & $n_{01}$ & $n_{00}$ \\
\hline
\end{tabular}

pressuposto de que a verificação do indivíduo independe do estado real da doença, Zhou (1998) definiu a função de verossimilhança para $(\boldsymbol{\psi}, \boldsymbol{\eta})$ como

$$
\begin{aligned}
L(\boldsymbol{\psi}, \boldsymbol{\eta})= & \prod_{i=0}^{1} \prod_{j=0}^{1}\left[P\left(D=1, T_{1}=i, T_{2}=j, V=1\right)\right]^{s_{i j}} \\
& \times \prod_{i=0}^{1} \prod_{j=0}^{1}\left[P\left(D=0, T_{1}=i, T_{2}=j, V=1\right)\right]^{r_{i j}} \\
& \times \prod_{i=0}^{1} \prod_{j=0}^{1}\left[P\left(T_{1}=i, T_{2}=j, V=0\right)\right]^{u_{i j}} .
\end{aligned}
$$

Já que há independência entre $D$ e $V$, tem-se que

$$
\begin{aligned}
L(\boldsymbol{\psi}, \boldsymbol{\eta})= & \prod_{i=0}^{1} \prod_{j=0}^{1}\left[P\left(D=1 \mid T_{1}=i, T_{2}=j\right) P\left(T_{1}=i, T_{2}=j\right)\right]^{s_{i j}} \\
& \times \prod_{i=0}^{1} \prod_{j=0}^{1}\left[P\left(D=0 \mid T_{1}=i, T_{2}=j\right) P\left(T_{1}=i, T_{2}=j\right)\right]^{r_{i j}} \\
& \times \prod_{i=0}^{1} \prod_{j=0}^{1}\left[P\left(T_{1}=i, T_{2}=j\right)\right]^{u_{i j}} .
\end{aligned}
$$

Utilizando a notação introduzida anteriormente, tem-se que

$$
L(\boldsymbol{\psi}, \boldsymbol{\eta})=\prod_{i=0}^{1} \prod_{j=0}^{1}\left[\psi_{i j} \eta_{i j}\right]^{s_{i j}} \times \prod_{i=0}^{1} \prod_{j=0}^{1}\left[\left(1-\psi_{i j}\right) \eta_{i j}\right]^{r_{i j}} \times \prod_{i=0}^{1} \prod_{j=0}^{1}\left[\eta_{i j}\right]^{u_{i j}}
$$

e, aplicando-se o logaritmo, chega-se em 


$$
\begin{aligned}
\ln L(\boldsymbol{\psi}, \boldsymbol{\eta}) & =l(\boldsymbol{\psi}, \boldsymbol{\eta})=\sum_{i=0}^{1} \sum_{j=0}^{1}\left[s_{i j} \ln \psi_{i j}+s_{i j} \ln \eta_{i j}+r_{i j} \ln \left(1-\psi_{i j}\right)+r_{i j} \ln \eta_{i j}+u_{i j} \ln \eta_{i j}\right] \\
& =\sum_{i=0}^{1} \sum_{j=0}^{1}\left[s_{i j} \ln \psi_{i j}+r_{i j} \ln \left(1-\psi_{i j}\right)\right]+\sum_{i=0}^{1} \sum_{j=0}^{1}\left[\left(s_{i j}+r_{i j}+u_{i j}\right) \ln \eta_{i j}\right] .
\end{aligned}
$$

Como $s_{i j}+r_{i j}+u_{i j}=n_{i j}$, tem-se que

$$
l(\boldsymbol{\psi}, \boldsymbol{\eta})=\sum_{i=0}^{1} \sum_{j=0}^{1}\left[s_{i j} \ln \psi_{i j}+r_{i j} \ln \left(1-\psi_{i j}\right)\right]+\sum_{i=0}^{1} \sum_{j=0}^{1}\left[n_{i j} \ln \eta_{i j}\right] .
$$

Os estimadores de máxima verossimilhança de $\psi_{i j}$ são encontrados a partir da expressão

$$
\frac{\partial \ln L(\boldsymbol{\psi}, \boldsymbol{\eta})}{\partial \psi_{i j}}=\frac{s_{i j}}{\psi_{i j}}-\frac{r_{i j}}{1-\psi_{i j}}=0
$$

Portanto,

$$
\widehat{\psi}_{i j}=\frac{s_{i j}}{s_{i j}+r_{i j}}
$$

Lembrando-se que $\eta_{00}>0, \eta_{01}>0, \eta_{10}>0$ e $\eta_{11}=1-\eta_{00}-\eta_{01}-\eta_{10}$, pode-se considerar que o termo

$$
\ell_{\eta}=\sum_{i=0}^{1} \sum_{j=0}^{1}\left[n_{i j} \ln \eta_{i j}\right]
$$

é a função de verossimilhança de uma distribuição multinomial. Portanto, os estimadores $\widehat{\eta}_{i j}$ de máxima verossimilhança de $\eta_{i j}$ são dados por

$$
\widehat{\eta}_{i j}=\frac{n_{i j}}{n}
$$

Considerando $\ell_{\psi}=\sum_{i=0}^{1} \sum_{j=0}^{1}\left[s_{i j} \ln \psi_{i j}+r_{i j} \ln \left(1-\psi_{i j}\right)\right]$, tem-se que $\ln L(\boldsymbol{\psi}, \boldsymbol{\eta})=$ $\ell_{\psi}+\ell_{\eta}$. Das expressões (49), (64) e (65), tem-se que o estimador $\widehat{\xi}$ da prevalência $\xi$ é dado por 


$$
\widehat{\xi}=\sum_{i=0}^{1} \sum_{j=0}^{1} \widehat{\psi}_{i j} \widehat{\eta}_{i j}=\sum_{i=0}^{1} \sum_{j=0}^{1} \frac{s_{i j}}{s_{i j}+r_{i j}} \frac{n_{i j}}{n} .
$$

Assim, os estimadores de máxima verossimilhança para $S_{E 1}$ e $S_{E 2}$ são dados, respectivamente, por

$$
\widehat{S}_{E 1}=\frac{\sum_{j=0}^{1} \widehat{\psi}_{1 j} \widehat{\eta}_{1 j}}{\widehat{\xi}}=\frac{\sum_{j=0}^{1} \frac{s_{1 j} n_{1 j}}{s_{1 j}+r_{1 j}}}{\sum_{i=0}^{1} \sum_{j=0}^{1} \frac{s_{i j} n_{i j}}{s_{i j}+r_{i j}}}
$$

e

$$
\widehat{S}_{E 2}=\frac{\sum_{i=0}^{1} \widehat{\psi}_{i 1} \widehat{\eta}_{i 1}}{\widehat{\xi}}=\frac{\sum_{i=0}^{1} \frac{s_{i 1} n i_{1}}{s_{i 1}+r_{i 1}}}{\sum_{i=0}^{1} \sum_{j=0}^{1} \frac{s_{i j} n_{i j}}{s_{i j}+r_{i j}}} .
$$

Por sua vez, os estimadores de máxima verossimilhança para $S_{P 1}$ e $S_{P 2}$ são dados, respectivamente, por

$$
\widehat{S}_{P 1}=\frac{\sum_{j=0}^{1}\left(1-\widehat{\psi}_{0 j}\right) \widehat{\eta}_{0 j}}{1-\widehat{\xi}}=\frac{\sum_{j=0}^{1} \frac{r_{0 j} n_{0 j}}{s_{0 j}+r_{0 j}}}{\sum_{i=0}^{1} \sum_{j=0}^{1} \frac{r_{i j} n_{i j}}{s_{i j}+r_{i j}}}
$$

e

$$
\widehat{S}_{P 2}=\frac{\sum_{i=0}^{1}\left(1-\widehat{\psi}_{i 0}\right) \widehat{\eta}_{i 0}}{1-\widehat{\xi}}=\frac{\sum_{i=0}^{1} \frac{r_{i 0} n_{i 0}}{s_{i 0}+r_{i 0}}}{\sum_{i=0}^{1} \sum_{j=0}^{1} \frac{r_{i j} n_{i j}}{s_{i j}+r_{i j}}}
$$

Considerando as razões de verossimilhanças $R V_{1}=S_{E 1} /\left(1-S_{P 1}\right)$ e $R V_{1}=S_{E 2} /(1-$ $S_{P 2}$ ), ou seja, o quociente entre a probabilidade de cada um dos dois testes classificar corretamente como positivo um indivíduo de fato portador da doença e a probabilidade do mesmo teste classificar erroneamente como positivo um indivíduo não doente, Nofuentes e del Castillo (2005) propuseram estimativas de máxima verossimilhança para estas medidas e um teste de hipóteses assintótico para compará-las. 


\subsection{Método Bayesiano (testes independentes)}

A seguir, será introduzido um método bayesiano para estimar-se as medidas de desempenho de dois testes diagnósticos na situação em que o viés de verificação está presente. Aqui, será admitido que o pressuposto de que $P\left(V \mid T_{1}, T_{2}, D\right)=P\left(V \mid T_{1}, T_{2}\right)$ é inválido, ou seja, a verificação depende do verdadeiro estado da doença. Assim, serão adicionados ao modelo os parâmetros $\lambda_{111}, \lambda_{101}, \lambda_{011}, \lambda_{001}, \lambda_{110}, \lambda_{100}, \lambda_{010}, \lambda_{000}$, definidos por

$$
\begin{aligned}
& \lambda_{111}=P\left(V \mid T_{1}=1, T_{2}=1, D=1\right) \\
& \lambda_{101}=P\left(V \mid T_{1}=1, T_{2}=0, D=1\right) \\
& \lambda_{011}=P\left(V \mid T_{1}=0, T_{2}=1, D=1\right) \\
& \lambda_{001}=P\left(V \mid T_{1}=0, T_{2}=0, D=1\right) \\
& \lambda_{110}=P\left(V \mid T_{1}=1, T_{2}=1, D=0\right) \\
& \lambda_{100}=P\left(V \mid T_{1}=1, T_{2}=0, D=0\right) \\
& \lambda_{010}=P\left(V \mid T_{1}=0, T_{2}=1, D=0\right) \\
& \lambda_{000}=P\left(V \mid T_{1}=0, T_{2}=0, D=0\right) .
\end{aligned}
$$

Também serão abordadas duas situações: uma primeira, em que assumir-se-á independência entre entre os testes $T_{1}$ e $T_{2}$ e outra em que há a dependência entre $T_{1}$ e $T_{2}$. Brenner (1996), Torrance - Rynard e Walter (1997) e Vacek (1985) discutem de uma maneira mais ampla a dependência entre testes diagnósticos.

Considerando-se que $T_{1}$ e $T_{2}$ são independentes, a Tabela 50 exibe todas as possíveis combinações entre as variáveis $V, D, T_{1}$ e $T_{2}$ e as suas contribuições à verossimilhança.

Na Tabela $50, u_{111}, u_{110}, u_{101}, u_{100}, u_{011}, u_{010}, u_{001}$ e $u_{000}$ são quantidades desconhecidas, tais que $u_{11}=u_{111}+u_{110}, u_{10}=u_{101}+u_{100}, u_{01}=u_{011}+u_{010}$ e $u_{00}=u_{001}+u_{000}$, sendo que $u_{11}, u_{10}, u_{01}$ e $u_{00}$ são conhecidos (ver Tabela 49). Nota-se que 
Tabela 50: Contribuições de todas as possíveis combinações entre as variáveis $V, D, T_{1}$ e $T_{2}$ à verossimilhança.

\begin{tabular}{cccccc}
\hline$T_{1}$ & $T_{2}$ & $D$ & $V$ & Freqüência & Contribuição à Verossimilhança \\
\hline 1 & 1 & 1 & 1 & $s_{11}$ & $\xi \lambda_{111} S_{E 1} S_{E 2}$ \\
1 & 0 & 1 & 1 & $s_{10}$ & $\xi \lambda_{101} S_{E 1}\left(1-S_{E 2}\right)$ \\
0 & 1 & 1 & 1 & $s_{01}$ & $\xi \lambda_{011}\left(1-S_{E 1}\right) S_{E 2}$ \\
0 & 0 & 1 & 1 & $s_{00}$ & $\xi \lambda_{001}\left(1-S_{E 1}\right)\left(1-S_{E 2}\right)$ \\
1 & 1 & 0 & 1 & $r_{11}$ & $(1-\xi) \lambda_{110}\left(1-S_{P 1}\right)\left(1-S_{P 2}\right)$ \\
1 & 0 & 0 & 1 & $r_{10}$ & $(1-\xi) \lambda_{100}\left(1-S_{P 1}\right) S_{P 2}$ \\
0 & 1 & 0 & 1 & $r_{01}$ & $(1-\xi) \lambda_{010} S_{P 1}\left(1-S_{P 2}\right)$ \\
0 & 0 & 0 & 1 & $r_{00}$ & $(1-\xi) \lambda_{000} S_{P 1} S_{P 2}$ \\
1 & 1 & 1 & 0 & {$\left[u_{111}\right]$} & $\xi\left(1-\lambda_{111}\right) S_{P 1} S_{P 2}$ \\
1 & 0 & 1 & 0 & {$\left[u_{101}\right]$} & $\xi\left(1-\lambda_{101}\right) S_{P 1}\left(1-S_{P 2}\right)$ \\
0 & 1 & 1 & 0 & {$\left[u_{011}\right]$} & $\xi\left(1-\lambda_{011}\right)\left(1-S_{P 1}\right) S_{P 2}$ \\
0 & 0 & 1 & 0 & {$\left[u_{001}\right]$} & $\xi\left(1-\lambda_{001}\right)\left(1-S_{P 1}\right)\left(1-S_{P 2}\right)$ \\
1 & 1 & 0 & 0 & {$\left[u_{110}\right]$} & $(1-\xi)\left(1-\lambda_{110}\right)\left(1-S_{P 1}\right)\left(1-S_{P 2}\right)$ \\
1 & 0 & 0 & 0 & {$\left[u_{100}\right]$} & $(1-\xi)\left(1-\lambda_{100}\right)\left(1-S_{P 1}\right) S_{P 2}$ \\
0 & 1 & 0 & 0 & {$\left[u_{010}\right]$} & $(1-\xi)\left(1-\lambda_{010}\right) S_{P 1}\left(1-S_{P 2}\right)$ \\
0 & 0 & 0 & 0 & {$\left[u_{000}\right]$} & $(1-\xi)\left(1-\lambda_{000}\right) S_{P 1} S_{P 2}$ \\
\hline
\end{tabular}

$P\left(T_{1}=1, T_{2}=1, D=1, V=1\right)=P(D=1) P\left(V=1 \mid T_{1}=1, T_{2}=1, D=1\right) P\left(T_{1}=1, T_{2}=1, D=1\right)$.

Se $T_{1}$ e $T_{2}$ são independentes, $P\left(T_{1}=1, T_{2}=1 \mid D=1\right)=P\left(T_{1}=1 \mid D=1\right) P\left(T_{2}=\right.$ $1 \mid D=1)=S_{E 1} S_{E 2}$, e $P\left(T_{1}=1, T_{2}=1, D=1, V=1\right)=\xi \lambda_{111} S_{E 1} S_{E 2}$. De forma análoga, pode-se obter as demais contribuições à verossimilhança exibidas na Tabela 50, dadas por $P\left(T_{1}=i, T_{2}=j, D=d, V=v\right)$, em que $i=0,1, j=0,1, d=0,1$, e $v=0,1$. A função de verossimilhança para $\boldsymbol{\theta}=\left(\xi, S_{E 1}, S_{E 2}, S_{P 1}, S_{P 2}, \lambda_{111}, \lambda_{101}, \lambda_{011}, \lambda_{001}, \lambda_{110}\right.$, $\left.\lambda_{100}, \lambda_{010}, \lambda_{000}\right)^{\prime}$ é dada por

$$
\begin{aligned}
L(\boldsymbol{\theta})= & \xi^{\Sigma_{i=0}^{1} \Sigma_{j=0}^{1}\left(s_{i j}+u_{i j 1}\right)}(1-\xi)^{\Sigma_{i=0}^{1} \Sigma_{j=0}^{1}\left(r_{i j}+u_{i j 0}\right)} S_{E 1}^{s_{11}+s_{10}+u_{111}+u_{101}}\left(1-S_{E 1}^{s_{01}+s_{00}+u_{011}+u_{001}}\right) \times \\
& S_{E 2}^{s_{11}+s_{10}+u_{111}+u_{011}}\left(1-S_{E 2}^{s_{10}+s_{00}+u_{101}+u_{001}}\right) S_{P 1}^{r_{01}+r_{00}+u_{010}+u_{000}}\left(1-S_{P 1}^{r_{11}+r_{10}+u_{110}+u_{100}}\right) \times \\
& S_{P 2}^{r_{10}+r_{00}+u_{100}+u_{000}}\left(1-S_{P 2}^{r_{11}+r_{01}+u_{110}+u_{010}}\right) \prod_{i-0}^{1} \prod_{j-0}^{1} \lambda_{i j 1}^{s_{i j}}\left(1-\lambda_{i j 1}\right)^{u_{i j 1}} \lambda_{i j 0}^{r_{i j}}\left(1-\lambda_{i j 0}\right)^{u_{i j 0}} .
\end{aligned}
$$


Sejam $Y_{1}, Y_{2}, Y_{3}$ e $Y_{4}$ variáveis referentes ao número de indivíduos doentes nãoverificados, sendo $Y_{1}$ especificicamente associada ao número de indivíduos com resultados positivos para ambos os testes; $Y_{2}$ associada ao número de indivíduos com resultado positivo para o teste 1 e negativo para o teste $2 ; Y_{3}$ associada ao número de indivíduos com resultado negativo para o teste 1 e positivo para o teste 2 ; e $Y_{4}$ associada ao número indivíduos com resultados negativos para ambos os testes. Essas variáveis, dentro da terminologia introduzida por Tanner e Wong (1987), são definidas como variáveis latentes, desde que exista a possibilidade de reproduzi-las através de suas distribuições de probabilidade, apesar de não serem observáveis. A partir da fórmula de Bayes, verifica-se que as distribuições condicionais de $Y_{1}, Y_{2}, Y_{3}$ e $Y_{4}$ são dadas por:

$$
\begin{gathered}
Y_{1} \quad \mid \quad u_{11}, \boldsymbol{\theta} \sim \text { Binomial }\left(u_{11 ;} \frac{\xi\left(1-\lambda_{111}\right) S_{E 1} S_{E 2}}{\xi\left(1-\lambda_{111}\right) S_{E 1} S_{E 2}+(1-\xi)\left(1-\lambda_{110}\right)\left(1-S_{P 1}\right)\left(1-S_{P 2}\right)}\right), \\
Y_{2} \quad \mid \quad u_{10}, \boldsymbol{\theta} \sim \operatorname{Binomial}\left(u_{10 ;} ; \frac{\xi\left(1-\lambda_{101}\right) S_{E 1}\left(1-S_{E 2}\right)}{\xi\left(1-\lambda_{101}\right) S_{E 1}\left(1-S_{E 2}\right)+(1-\xi)\left(1-\lambda_{100}\right)\left(1-S_{P 1}\right) S_{P 2}}\right), \\
Y_{3} \quad \mid \quad u_{01}, \boldsymbol{\theta} \sim \text { Binomial }\left(u_{01} ; \frac{\xi\left(1-\lambda_{011}\right)\left(1-S_{E 1}\right) S_{E 2}}{\xi\left(1-\lambda_{011}\right)\left(1-S_{E 1}\right) S_{E 2}+(1-\xi)\left(1-\lambda_{010}\right) S_{P 1}\left(1-S_{P 2}\right)}\right), \\
Y_{4} \quad \mid \quad u_{00}, \boldsymbol{\theta} \sim \text { Binomial }\left(u_{00} ; \frac{\xi\left(1-\lambda_{001}\right)\left(1-S_{E 1}\right)\left(1-S_{E 2}\right)}{\xi\left(1-\lambda_{001}\right)\left(1-S_{E 1}\right)\left(1-S_{E 2}\right)+(1-\xi)\left(1-\lambda_{000}\right) S_{P 1} S_{P 2}}\right) .
\end{gathered}
$$

Na análise bayesiana assume-se que as densidades a priori para todos os parâmetros do vetor $\boldsymbol{\theta}$ possuem distribuição possuem distribuição Beta, em que $\alpha_{\theta}$ e $\beta_{\theta}$ são hiperparâmetros (conhecidos) da distribuição de $\boldsymbol{\theta}$. Seja $\mathbf{Z}=\left(Y_{1}, Y_{2}, Y_{3}, Y_{4}\right)^{\prime}$ um vetor de variáveis não-observáveis. Combinando-se a função de verossimilhança com as densidades a priori, as distribuições condicionais para o algoritmo de amostradores de Gibbs (SMITH e ROBERTS, 1993) são dadas por (74) e por:

$$
\begin{gathered}
\xi \quad \mathbf{Z}, \mathbf{Y}, \alpha_{\xi}, \beta_{\xi} \sim \operatorname{Beta}\left(\sum_{i=0}^{1} \sum_{j=0}^{1} s_{i j}+\sum_{k=1}^{4} Y_{K}+\alpha_{\xi} ; \sum_{i=0}^{1} \sum_{j=0}^{1}\left(r_{i j}+u_{i j}\right)-\sum_{k=1}^{4} Y_{K}+\beta_{\xi}\right), \\
S_{E 1} \mid \mathbf{Z}, \mathbf{Y}, \alpha_{S_{E 1}}, \beta_{S_{E 1}} \sim \operatorname{Beta}\left(s_{11}+s_{10}+Y_{1}+Y_{2}+\alpha_{S_{E 1}} ; s_{01}+s_{00}+Y_{3}+Y_{4}+\beta_{S_{E 1}}\right), \\
S_{E 2} \mid \mathbf{Z}, \mathbf{Y}, \alpha_{S_{E 2}}, \beta_{S_{E 2}} \sim \operatorname{Beta}\left(s_{11}+s_{01}+Y_{1}+Y 3+\alpha_{S_{E 2}} ; s_{10}+s_{00}+Y_{2}+Y_{4}+\beta_{S 2}\right), \\
S_{P 1} \mid \mathbf{Z}, \mathbf{Y}, \alpha_{S_{P 1}}, \beta_{S_{P 1}} \sim \operatorname{Beta}\left(r_{01}+r_{00}+u_{01}+u_{00}-Y_{3}-Y_{4}+\alpha_{S_{P 1}} ;\right. \\
\left.r_{11}+r_{10}+u_{11}+u_{10}-Y_{1}-Y_{2}+\beta_{S_{P 1}}\right),
\end{gathered}
$$




$$
\begin{array}{l|l}
S_{P 2} \mid \mathbf{Z}, \mathbf{Y}, \alpha_{S_{P 2}}, \beta_{S_{P 2}} \sim \operatorname{Beta}\left(r_{10}+r_{00}+u_{10}+u_{00}-Y_{2}-Y_{4}+\alpha_{S_{P 2}} ;\right. & \left.r_{11}+r_{01}+u_{11}+u_{01}-Y_{1}-Y_{3}+\beta_{S_{P 2}}\right), \\
\lambda_{111} \mid \mathbf{Z}, \mathbf{Y}, \alpha_{\lambda_{111}}, \beta_{\lambda_{111}} \sim \operatorname{Beta}\left(s_{11}+\alpha_{\lambda_{111}} ; Y_{1}+\beta_{\lambda_{111}}\right), \\
\lambda_{101} \mid \mathbf{Z}, \mathbf{Y}, \alpha_{\lambda_{101}}, \beta_{\lambda_{101}} \sim \operatorname{Beta}\left(s_{10}+\alpha_{\lambda_{101}} ; Y_{2}+\beta_{\lambda_{101}}\right), \\
\lambda_{011} \mid \mathbf{Z}, \mathbf{Y}, \alpha_{\lambda_{011}}, \beta_{\lambda_{011}} \sim \operatorname{Beta}\left(s_{01}+\alpha_{\lambda_{011}} ; Y_{3}+\beta_{\lambda_{011}}\right), \\
\lambda_{001} \mid \mathbf{Z}, \mathbf{Y}, \alpha_{\lambda_{001}}, \beta_{\lambda_{001}} \sim \operatorname{Beta}\left(s_{00}+\alpha_{\lambda_{001}} ; Y_{4}+\beta_{\lambda_{001}}\right), \\
\lambda_{110} \mid \mathbf{Z}, \mathbf{Y}, \alpha_{\lambda_{110}}, \beta_{\lambda_{110}} \sim \operatorname{Beta}\left(r_{11}+\alpha_{\lambda_{110}} ; u_{11}-Y_{1}+\beta_{\lambda_{110}}\right), \\
\lambda_{100} \mid \mathbf{Z}, \mathbf{Y}, \alpha_{\lambda_{100}}, \beta_{\lambda_{100}} \sim \operatorname{Beta}\left(r_{10}+\alpha_{\lambda_{100}} ; u_{10}-Y_{2}+\beta_{\lambda_{100}}\right), \\
\lambda_{010} \mid \mathbf{Z}, \mathbf{Y}, \alpha_{\lambda_{010}}, \beta_{\lambda_{010}} \sim \operatorname{Beta}\left(r_{01}+\alpha_{\lambda_{010}} ; u_{01}-Y_{3}+\beta_{\lambda_{010}}\right), \\
\lambda_{000} \mid \mathbf{Z}, \mathbf{Y}, \alpha_{\lambda_{000}}, \beta_{\lambda_{000}} \sim \operatorname{Beta}\left(r_{00}+\alpha_{\lambda_{000}} ; u_{00}-Y_{4}+\beta_{\lambda_{000}}\right) .
\end{array}
$$

O primeiro passo do algoritmo consiste em gerar aleatoriamente $Y_{1}, Y_{2}, Y_{3}$ e $Y_{4}$ a partir de (74), dando valores arbitrários para os parâmetros do vetor $\boldsymbol{\theta}$. A seguir, utilizando-se os valores obtidos para $Y_{1}, Y_{2}, Y_{3}$ e $Y_{4}$, observações de $\xi, S_{E 1}, S_{E 2}, S_{P 1}, S_{P 2}, \lambda_{111}, \lambda_{101}, \lambda_{011}$, $\lambda_{001}, \lambda_{110}, \lambda_{100}, \lambda_{010}, \lambda_{000}$ são geradas aleatoriamente conforme suas respectivas distribuições condicionais (75). Os valores, assim obtidos, são usados para gerar, aleatoriamente, novos valores de $Y_{1}, Y_{2}, Y_{3}$ e $Y_{4}$, que, por sua vez, são utilizados para gerar novos valores de $\xi, S_{E 1}, S_{E 2}, S_{P 1}, S_{P 2}, \lambda_{111}, \lambda_{101}, \lambda_{011}, \lambda_{001}, \lambda_{110}, \lambda_{100}, \lambda_{010}, \lambda_{000}$, e este ciclo repete-se por um número grande de vezes até que haja convergência. As amostras aleatórias geradas são usadas para se estimar as densidades marginais a posteriori de cada parâmetro de $\boldsymbol{\theta}$ e das variáveis $Y_{1}, Y_{2}, Y_{3}$ e $Y_{4}$.

Um exemplo real de aplicação da metodologia proposta é o caso apresentado por Hall et al. (1996), o qual trata de um estudo epidemiológico sobre demência para investigar fatores de risco para a doença de Alzheimer. Um dos objetivos do estudo é comparar a acurácia de um novo teste diagnóstico com a de um teste padrão de rastreamento. $\mathrm{O}$ diagnóstico de demência é estabelecido através de uma avaliação clínica que envolve exames neurológicos, uma bateria de testes neuropsicológicos, testes de laboratório, tomografia computadorizada e uma entrevista detalhada. Pelo fato de haver tempo escasso e os custos 
serem altos, nem todos os indivíduos rastreados foram avaliados clinicamente. A Tabela 51 mostra as freqüências obtidas.

Tabela 51: Dados do estudo de HALL et al. (1996) sobre o diagnóstico da doença de Alzheimer.

\begin{tabular}{cccccc}
\hline & \multicolumn{2}{c}{$T_{1}=1$} & & \multicolumn{2}{c}{$T_{1}=0$} \\
\cline { 2 - 3 } \cline { 5 - 6 } & $T_{2}=1$ & $T_{2}=0$ & & $T_{2}=1$ & $T_{2}=0$ \\
\hline$V=1, D=1$ & 38 & 5 & & 3 & 1 \\
$V=1, D=0$ & 35 & 29 & & 25 & 89 \\
$V=0$ & 31 & 17 & & 117 & 1105 \\
Total & 104 & 51 & & 145 & 1185 \\
\hline
\end{tabular}

Como outro exemplo, pode-se citar o estudo conduzido por Ratnam et al. (2000), que objetivou comparar os desempenhos do teste de HPV $\left(T_{1}\right)$ e do exame convencional de Papanicolaou $\left(T_{2}\right)$ na detecção do câncer cervical em mulheres com idade entre 18 e 69 anos, residentes em uma localidade do Canadá. Convencionou-se classificar o resultado do teste de HPV como positivo quando detectados tipos virais definidos como de alto risco, e classificar o resultado do exame de Papanicolaou como positivo, quando observadas lesões intraepiteliais de baixo grau ou mais graves. O padrão ouro foi definido pelo resultado da colposcopia ou histologia. Os resultados do estudo aparecem na Tabela 52, em que observa-se que 19,4\% das 2098 mulheres amostradas foram verificadas pelo padrão ouro.

Tabela 52: Dados do estudo de RATNAM et al. (2000) sobre o rastreamento do câncer cervical.

\begin{tabular}{cccccc}
\hline & \multicolumn{2}{c}{$T_{1}=1$} & & \multicolumn{2}{c}{$T_{1}=0$} \\
\cline { 2 - 3 } \cline { 5 - 6 } & $T_{2}=1$ & $T_{2}=0$ & & $T_{2}=1$ & $T_{2}=0$ \\
\hline$V=1, D=1$ & 34 & 71 & & 14 & 50 \\
$V=1, D=0$ & 23 & 58 & & 15 & 143 \\
$V=0$ & 4 & 37 & & 2 & 1647 \\
Total & 61 & 166 & & 31 & 1840 \\
\hline
\end{tabular}

\subsubsection{Simulações}

Considerando os dados do estudo de Hall et al. (1996), algumas simulações foram feitas, afim de se avaliar o desempenho do método bayesiano na estimação dos parâmetros de performance de testes diagnósticos. Utilizando o software WinBugs, versão 1.4 
(SPIEGELHALTER et al, 2001), foram geradas 205 mil amostras, das quais as primeiras 5000 foram descartadas para que as inferências estivessem livres dos efeitos dos valores iniciais. Também foram consideradas apenas as $15^{a}, 30^{a}, 45^{a}, \ldots$ iterações, resultando em, aproximadamente, 13 mil amostras de Gibbs. A convergência do algoritmo foi verificada a partir de gráficos temporais das amostras geradas e utilizando-se técnicas usuais existentes na literatura (GELMAN e RUBIN, 1992). Estimativas de máxima verossimilhança e os intervalos de confiança $95 \%$ para a sensibilidade do teste 1 e do teste 2 são, respectivamente, $68,1 \%(44,1 \% ; 92,2 \%)$ e $77,0 \%(53,6 \% ; 100,0 \%)$ e, para a especificidade são iguais a $93,4 \%(92,0 \% ; 95,0 \%)$ e $87,2 \%(85,2 \% ; 89,3 \%)$, respectivamente. A prevalência de de demência é estimada em 6,1\%, numa amostra de 1485 indivíduos. Na Tabela 53, foram consideradas distribuições a priori, Beta $(0,5 ; 0,5)$, não informativas para todos os parâmetros do vetor $\boldsymbol{\theta}$. Os resultados são dados por:

Tabela 53: Resultados a posteriori para os dados de HALL et al. (1996) - distribuições a priori não informativas.

\begin{tabular}{|c|c|c|c|c|c|}
\hline \multirow[b]{2}{*}{ Parâmetro } & \multicolumn{3}{|c|}{ Informações a priori } & \multicolumn{2}{|c|}{ Informações a posteriori } \\
\hline & Distribuições & Média (\%) & $\mathrm{DP}^{(a)}(\%)$ & Média (\%) & ICr $95 \%$ \\
\hline$\xi$ & $\operatorname{Beta}(0,5 ; 0,5)$ & 50,00 & 35,30 & 84,79 & $(82,39 ; 87,01)$ \\
\hline$S_{E 1}$ & $\operatorname{Beta}(0,5 ; 0,5)$ & 50,00 & 35,30 & 4,75 & $(3,65 ; 6,00)$ \\
\hline$S_{E 2}$ & $\operatorname{Beta}(0,5 ; 0,5)$ & 50,00 & 35,30 & 10,70 & $(8,56 ; 12,98)$ \\
\hline$S_{P 1}$ & $\operatorname{Beta}(0,5 ; 0,5)$ & 50,00 & 35,30 & 57,47 & $(49,69 ; 64,89)$ \\
\hline$S_{P 2}$ & $\operatorname{Beta}(0,5 ; 0,5)$ & 50,00 & 35,30 & 49,21 & $(41,49 ; 57,18)$ \\
\hline$\lambda_{111}$ & $\operatorname{Beta}(0,5 ; 0,5)$ & 50,00 & 35,30 & 98,38 & $(91,91 ; 100,00)$ \\
\hline$\lambda_{011}$ & $\operatorname{Beta}(0,5 ; 0,5)$ & 50,00 & 35,30 & 3,65 & $(0,87 ; 8,42)$ \\
\hline$\lambda_{101}$ & $\operatorname{Beta}(0,5 ; 0,5)$ & 50,00 & 35,30 & 24,86 & $(9,62 ; 44,84)$ \\
\hline$\lambda_{001}$ & $\operatorname{Beta}(0,5 ; 0,5)$ & 50,00 & 35,30 & 0,13 & $(0,01 ; 0,42)$ \\
\hline$\lambda_{110}$ & $\operatorname{Beta}(0,5 ; 0,5)$ & 50,00 & 35,30 & 53,10 & $(41,11 ; 64,90)$ \\
\hline$\lambda_{010}$ & $\operatorname{Beta}(0,5 ; 0,5)$ & 50,00 & 35,30 & 53,39 & $(31,65 ; 85,78)$ \\
\hline$\lambda_{100}$ & $\operatorname{Beta}(0,5 ; 0,5)$ & 50,00 & 35,30 & 96,03 & $(82,34 ; 99,99)$ \\
\hline$\lambda_{000}$ & $\operatorname{Beta}(0,5 ; 0,5)$ & 50,00 & 35,30 & 97,00 & $(85,90 ; 100,00)$ \\
\hline
\end{tabular}

${ }^{(a)}$ desvio padrão

Na Tabela 54 foram consideradas distribuições a priori, Beta $(0,5 ; 0,5)$, não informativas para todos os parâmetros $\lambda_{i j d}(i=0,1 ; j=0,1 ; d=0,1)$, e informativas para $S_{E 1}$, $S_{E 2}, S_{P 1}, S_{P 2}$ e $\xi$ 
Tabela 54: Resultados a posteriori para os dados de HALL et al. (1996).

\begin{tabular}{|c|c|c|c|c|c|}
\hline \multirow[b]{2}{*}{ Parâmetro } & \multicolumn{3}{|c|}{ Informações a priori } & \multicolumn{2}{|c|}{ Informações a posteriori } \\
\hline & Distribuições & Média (\%) & $\mathrm{DP}^{(a)}(\%)$ & Média (\%) & ICr $95 \%$ \\
\hline$\xi$ & $\operatorname{Beta}(1,85 ; 7,40)$ & 20,00 & 12,50 & 5,75 & $(4,49 ; 7,27)$ \\
\hline$S_{E 1}$ & $\operatorname{Beta}(8,25 ; 2,75)$ & 75,00 & 12,50 & 87,85 & $(74,76 ; 95,75)$ \\
\hline$S_{E 2}$ & $\operatorname{Beta}(8,25 ; 2,75)$ & 75,00 & 12,50 & 89,09 & $(80,81 ; 95,09)$ \\
\hline$S_{P 1}$ & $\operatorname{Beta}(8,25 ; 2,75)$ & 75,00 & 12,50 & 94,14 & $(92,84 ; 95,34)$ \\
\hline$S_{P 2}$ & $\operatorname{Beta}(10,00 ; 1,00)$ & 90,90 & 8,29 & 87,71 & $(85,87 ; 89,48)$ \\
\hline$\lambda_{111}$ & $\operatorname{Beta}(0,5 ; 0,5)$ & 50,00 & 35,30 & 55,25 & $(43,65 ; 66,68)$ \\
\hline$\lambda_{011}$ & $\operatorname{Beta}(0,5 ; 0,5)$ & 50,00 & 35,30 & 52,47 & $(9,66 ; 99,67)$ \\
\hline$\lambda_{101}$ & $\operatorname{Beta}(0,5 ; 0,5)$ & 50,00 & 35,30 & 80,11 & $(34,12 ; 99,96)$ \\
\hline$\lambda_{001}$ & $\operatorname{Beta}(0,5 ; 0,5)$ & 50,00 & 35,30 & 66,31 & $(8,42 ; 99,92)$ \\
\hline$\lambda_{110}$ & $\operatorname{Beta}(0,5 ; 0,5)$ & 50,00 & 35,30 & 98,04 & $(90,43 ; 100,00)$ \\
\hline$\lambda_{010}$ & $\operatorname{Beta}(0,5 ; 0,5)$ & 50,00 & 35,30 & 18,48 & $(12,36 ; 25,65)$ \\
\hline$\lambda_{100}$ & $\operatorname{Beta}(0,5 ; 0,5)$ & 50,00 & 35,30 & 64,60 & $(49,52 ; 79,01)$ \\
\hline$\lambda_{000}$ & $\operatorname{Beta}(0,5 ; 0,5)$ & 50,00 & 35,30 & 6,71 & $(5,35 ; 8,21)$ \\
\hline
\end{tabular}

(a) desvio padrão

Na Tabela 55 foram consideradas distribuições a priori informativas $S_{E 1}, S_{E 2}, S_{P 1}$, $S_{P 2}, \xi$ e $\lambda_{i j d}(i=0,1 ; j=0,1 ; d=0,1)$.

Tabela 55: Resultados a posteriori para os dados de HALL et al. (1996).

\begin{tabular}{|c|c|c|c|c|c|}
\hline \multirow[b]{2}{*}{ Parâmetro } & \multicolumn{3}{|c|}{ Informações a priori } & \multicolumn{2}{|c|}{ Informações a posteriori } \\
\hline & Distribuições & Média (\%) & $\mathrm{DP}^{(a)}(\%)$ & Média (\%) & ICr $95 \%$ \\
\hline$\xi$ & $\operatorname{Beta}(1,85 ; 7,40)$ & 20,00 & 12,50 & 5,88 & $(5,84 ; 7,37)$ \\
\hline$S_{E 1}$ & $\operatorname{Beta}(8,25 ; 2,75)$ & 75,00 & 12,50 & 86,66 & $(74,21 ; 94,88)$ \\
\hline$S_{E 2}$ & $\operatorname{Beta}(8,25 ; 2,75)$ & 75,00 & 12,50 & 87,55 & $(78,57 ; 94,23)$ \\
\hline$S_{P 1}$ & $\operatorname{Beta}(8,25 ; 2,75)$ & 75,00 & 12,50 & 94,18 & $(92,88 ; 95,37)$ \\
\hline$S_{P 2}$ & $\operatorname{Beta}(10,00 ; 1,00)$ & 90,90 & 8,29 & 87,70 & $(85,85 ; 89,47)$ \\
\hline$\lambda_{111}$ & $\operatorname{Beta}(8,10 ; 2,00)$ & 80,0 & 12,50 & 58,83 & $(47,68 ; 69,48)$ \\
\hline$\lambda_{011}$ & $\operatorname{Beta}(2,6 ; 2,6)$ & 50,00 & 20,00 & 45,41 & $(14,81 ; 82,90)$ \\
\hline$\lambda_{101}$ & $\operatorname{Beta}(2,6 ; 2,6)$ & 50,00 & 20,00 & 62,36 & $(30,48 ; 90,70)$ \\
\hline$\lambda_{001}$ & $\operatorname{Beta}(2,00 ; 8,10)$ & 20,00 & 12,50 & 24,76 & $(5,83 ; 51,60)$ \\
\hline$\lambda_{110}$ & $\operatorname{Beta}(8,10 ; 2,00)$ & 80,0 & 12,50 & 94,21 & $(84,91 ; 99,24)$ \\
\hline$\lambda_{010}$ & $\operatorname{Beta}(2,6 ; 2,6)$ & 50,00 & 20,00 & 19,44 & $(13,29 ; 26,48)$ \\
\hline$\lambda_{100}$ & $\operatorname{Beta}(2,6 ; 2,6)$ & 50,00 & 20,00 & 64,53 & $(50,14 ; 78,57)$ \\
\hline$\lambda_{000}$ & $\operatorname{Beta}(2,00 ; 8,10)$ & 20,00 & 12,50 & 6,79 & $(5,44 ; 8,31)$ \\
\hline
\end{tabular}

(a) desvio padrão

Na Tabela 56 foram consideradas distribuições a priori, Beta $(0,5 ; 0,5)$, não informativas para todos os parâmetros $\lambda_{i j d}(i=0,1 ; j=0,1 ; d=0,1)$ e $\xi$, e informativas para $S_{E 1}, S_{E 2}, S_{P 1}$ e $S_{P 2}$. 
Tabela 56: Resultados a posteriori para os dados de HALL et al. (1996).

\begin{tabular}{|c|c|c|c|c|c|}
\hline \multirow[b]{2}{*}{ Parâmetro } & \multicolumn{3}{|c|}{ Informações a priori } & \multicolumn{2}{|c|}{ Informações a posteriori } \\
\hline & Distribuições & Média (\%) & $\mathrm{DP}^{(a)}(\%)$ & Média (\%) & ICr $95 \%$ \\
\hline$\xi$ & $\operatorname{Beta}(0,5 ; 0,5)$ & 50,00 & 35,30 & 5,65 & $(4,40 ; 7,13)$ \\
\hline$S_{E 1}$ & $\operatorname{Beta}(8,25 ; 2,75)$ & 75,00 & 12,50 & 88,19 & $(75,68 ; 95,91)$ \\
\hline$S_{E 2}$ & $\operatorname{Beta}(8,25 ; 2,75)$ & 75,00 & 12,50 & 89,14 & $(80,76 ; 95,04)$ \\
\hline$S_{P 1}$ & $\operatorname{Beta}(8,25 ; 2,75)$ & 75,00 & 12,50 & 94,14 & $(92,81 ; 95,34)$ \\
\hline$S_{P 2}$ & $\operatorname{Beta}(10,00 ; 1,00)$ & 90,90 & 8,29 & 87,69 & $(85,79 ; 89,46)$ \\
\hline$\lambda_{111}$ & $\operatorname{Beta}(0,5 ; 0,5)$ & 50,00 & 35,30 & 55,22 & $(43,72 ; 66,70)$ \\
\hline$\lambda_{011}$ & $\operatorname{Beta}(0,5 ; 0,5)$ & 50,00 & 35,30 & 53,78 & $(9,91 ; 99,60)$ \\
\hline$\lambda_{101}$ & $\operatorname{Beta}(0,5 ; 0,5)$ & 50,00 & 35,30 & 19,15 & $(33,45 ; 99,97)$ \\
\hline$\lambda_{001}$ & $\operatorname{Beta}(0,5 ; 0,5)$ & 50,00 & 35,30 & 67,16 & $(9,18 ; 99,92)$ \\
\hline$\lambda_{110}$ & $\operatorname{Beta}(0,5 ; 0,5)$ & 50,00 & 35,30 & 98,06 & $(90,41 ; 100,00)$ \\
\hline$\lambda_{010}$ & $\operatorname{Beta}(0,5 ; 0,5)$ & 50,00 & 35,30 & 18,32 & $(12,34 ; 25,26)$ \\
\hline$\lambda_{100}$ & $\operatorname{Beta}(0,5 ; 0,5)$ & 50,00 & 35,30 & 64,59 & $(49,84 ; 79,03)$ \\
\hline$\lambda_{000}$ & $\operatorname{Beta}(0,5 ; 0,5)$ & 50,00 & 35,30 & 6,71 & $(5,37 ; 8,20)$ \\
\hline
\end{tabular}

(a) desvio padrão

Na Tabela 57 foram consideradas distribuições a priori, Beta $(0,5 ; 0,5)$, não informativas para $S_{E 1}, S_{E 2}, S_{P 1}, S_{P 2}$ e $\xi$, e informativas para $\lambda_{i j d}(i=0,1 ; j=0,1 ; d=0$, 1).

Tabela 57: Resultados a posteriori para os dados de HALL et al. (1996).

\begin{tabular}{|c|c|c|c|c|c|}
\hline \multirow[b]{2}{*}{ Parâmetro } & \multicolumn{3}{|c|}{ Informações a priori } & \multicolumn{2}{|c|}{ Informações a posteriori } \\
\hline & Distribuições & Média (\%) & $\mathrm{DP}^{(a)}(\%)$ & Média (\%) & ICr $95 \%$ \\
\hline$\xi$ & $\operatorname{Beta}(0,5 ; 0,5)$ & 50,00 & 35,30 & 82,47 & $(79,18 ; 85,26)$ \\
\hline$S_{E 1}$ & $\operatorname{Beta}(0,5 ; 0,5)$ & 50,00 & 35,30 & 4,72 & $(3,54 ; 6,03)$ \\
\hline$S_{E 2}$ & $\operatorname{Beta}(0,5 ; 0,5)$ & 50,00 & 35,30 & 10,44 & $(8,28 ; 12,69)$ \\
\hline$S_{P 1}$ & $\operatorname{Beta}(0,5 ; 0,5)$ & 50,00 & 35,30 & 62,22 & $(54,34 ; 69,72)$ \\
\hline$S_{P 2}$ & $\operatorname{Beta}(0,5 ; 0,5)$ & 50,00 & 35,30 & 53,26 & $(45,42 ; 61,18)$ \\
\hline$\lambda_{111}$ & $\operatorname{Beta}(8,10 ; 2,00)$ & 80,00 & 12,50 & 94,92 & $(86,24 ; 99,35)$ \\
\hline$\lambda_{011}$ & $\operatorname{Beta}(2,6 ; 2,6)$ & 50,00 & 20,00 & 6,03 & $(5,66 ; 12,06)$ \\
\hline$\lambda_{101}$ & $\operatorname{Beta}(2,6 ; 2,6)$ & 50,00 & 20,00 & 31,83 & $(14,94 ; 52,90)$ \\
\hline$\lambda_{001}$ & $\operatorname{Beta}(2,00 ; 8,10)$ & 20,00 & 12,50 & 0,28 & $(0,06 ; 0,68)$ \\
\hline$\lambda_{110}$ & $\operatorname{Beta}(8,10 ; 2,00)$ & 80,00 & 12,50 & 57,07 & $(45,71 ; 68,12)$ \\
\hline$\lambda_{010}$ & $\operatorname{Beta}(2,6 ; 2,6)$ & 50,00 & 20,00 & 46,31 & $(29,14 ; 68,42)$ \\
\hline$\lambda_{100}$ & $\operatorname{Beta}(2,6 ; 2,6)$ & 50,00 & 20,00 & 84,89 & $(67,71 ; 96,70)$ \\
\hline$\lambda_{000}$ & $\operatorname{Beta}(2,00 ; 8,10)$ & 20,00 & 12,50 & 70,19 & $(53,39 ; 84,92)$ \\
\hline
\end{tabular}

(a) desvio padrão

Observando que dentre os 1485 indivíduos amostrados, 215 foram verificados, foram especificadas as probabilidades de verificação, iguais e constantes, dadas por $\lambda_{111}=\lambda_{101}=$ $\lambda_{011}=\lambda_{001}=\lambda_{110}=\lambda_{100}=\lambda_{010}=\lambda_{000}=215 / 1485=14,5 \%$. Foram consideradas distribuições a priori não informativas para $S_{E 1}, S_{E 2}, S_{P 1}, S_{P 2}$ e $\xi$. Os resultados estão 
na Tabela 58

Tabela 58: Resultados a posteriori para os dados de HALL et al. (1996).

\begin{tabular}{cllllll}
\hline & \multicolumn{2}{l}{ Informações $a$ priori } & & \multicolumn{2}{l}{ Informações a posteriori } \\
\cline { 2 - 4 } \cline { 7 - 7 } Parâmetro & Distribuições & Média $(\%)$ & $\operatorname{DP}^{(a)}(\%)$ & & Média $(\%)$ & ICr $95 \%$ \\
\hline$\xi$ & Beta $(0,5 ; 0,5)$ & 50,00 & 35,30 & & 5,79 & \\
$S_{E 1}$ & Beta $(0,5 ; 0,5)$ & 50,00 & 35,30 & & 83,24 & $(4,44 ; 7,34)$ \\
$S_{E 2}$ & Beta $(0,5 ; 0,5)$ & 50,00 & 35,30 & & 88,80 & $(79,17 ; 94,21)$ \\
$S_{P 1}$ & Beta $(0,5 ; 0,5)$ & 50,00 & 35,30 & & 93,98 & $(92,64 ; 95,38)$ \\
$S_{P 2}$ & Beta $(0,5 ; 0,5)$ & 50,00 & 35,30 & & 87,63 & $(85,75 ; 89,42)$ \\
$\lambda_{i j d}^{(b)}$ & constante & 14,50 & - & & - & - \\
\hline
\end{tabular}

(a) desvio padrão; ${ }^{(b)} i=0,1 ; j=0,1 ; d=0,1$.

Na Tabela 59 foram consideradas distribuições a priori não informativas para $S_{E 1}$, $S_{E 2}, S_{P 1}, S_{P 2}$ e $\xi$, e ainda, valores constantes para $\lambda_{i j d}(i=0,1 ; j=0,1 ; d=0,1)$.

Tabela 59: Resultados a posteriori para os dados de HALL et al. (1996).

\begin{tabular}{cllllll}
\hline & \multicolumn{2}{l}{ Informações $a$ priori } & & \multicolumn{2}{c}{ Informações a posteriori } \\
\cline { 2 - 4 } Parâmetro & Distribuições & Média $(\%)$ & $\operatorname{DP}^{(a)}(\%)$ & & Média $(\%)$ & ICr $95 \%$ \\
\hline$\xi$ & Beta $(0,5 ; 0,5)$ & 50,00 & 35,30 & & 58,88 & $(4,51 ; 7,44)$ \\
$S_{E 1}$ & Beta $(8,25 ; 2,75)$ & 75,00 & 12,50 & & 81,51 & $(68,87 ; 91,77)$ \\
$S_{E 2}$ & Beta $(8,25 ; 2,75)$ & 75,00 & 12,50 & & 87,17 & $(78,54 ; 93,84)$ \\
$S_{P 1}$ & Beta $(8,25 ; 2,75)$ & 75,00 & 12,50 & & 93,86 & $(92,50 ; 95,09)$ \\
$S_{P 2}$ & Beta $(10,00 ; 1,00)$ & 90,90 & 8,29 & & 87,71 & $(85,87 ; 95,09)$ \\
$\lambda_{i j d}^{(b)}$ & constante & 14,50 & - & & - & - \\
\hline
\end{tabular}

${ }^{(a)}$ desvio padrão; ${ }^{(b)} i=0,1 ; j=0,1 ; d=0,1$.

Agora, considerando os dados apresentados por Ratnam et al. (2000), foram elaboradas algumas simulações. Estimativas de máxima verossimilhança e os intervalos de confiança $95 \%$ para a sensibilidade do teste de HPV e do exame de Papanicolaou são, respectivamente, $20,6 \%(15,7 \% ; 25,5 \%)$ e $8,3 \%(5,7 \% ; 10,9 \%)$ e, para a especificidade são iguais a $93,3 \%(91,9 \% ; 94,7 \%)$ e $97,3 \%(96,4 \% ; 98,1 \%)$, respectivamente. A prevalência de câncer cervical é estimada em 29,5\% entre as mulheres que atenderam aos critérios de inclusão no estudo. Foram consideradas as mesmas situações do exemplo de Hall et al. (1996). Na Tabela 60, foram consideradas distribuições a priori, Beta $(0,5 ; 0,5)$, não informativas para todos os parâmetros do vetor $\boldsymbol{\theta}$. 
Tabela 60: Resultados a posteriori para os dados de RATNAM et al. (2000) - distribuições a priori não informativas.

\begin{tabular}{|c|c|c|c|c|c|}
\hline \multirow[b]{2}{*}{ Parâmetro } & \multicolumn{3}{|c|}{ Informações a priori } & \multicolumn{2}{|c|}{ Informações a posteriori } \\
\hline & Distribuições & Média (\%) & $\mathrm{DP}^{(a)}(\%)$ & Média (\%) & ICr $95 \%$ \\
\hline$\xi$ & $\operatorname{Beta}(0,5 ; 0,5)$ & 50,00 & 35,30 & 87,83 & $(85,93 ; 89,40)$ \\
\hline$S_{E 1}$ & $\operatorname{Beta}(0,5 ; 0,5)$ & 50,00 & 35,30 & 7,50 & $(6,18 ; 8,84)$ \\
\hline$S_{E 2}$ & $\operatorname{Beta}(0,5 ; 0,5)$ & 50,00 & 35,30 & 2,72 & $(2,02 ; 3,52)$ \\
\hline$S_{P 1}$ & $\operatorname{Beta}(0,5 ; 0,5)$ & 50,00 & 35,30 & 64,93 & $(57,90 ; 71,69)$ \\
\hline$S_{P 2}$ & $\operatorname{Beta}(0,5 ; 0,5)$ & 50,00 & 35,30 & 83,15 & $(78,08 ; 87,77)$ \\
\hline$\lambda_{111}$ & $\operatorname{Beta}(0,5 ; 0,5)$ & 50,00 & 35,30 & 98,15 & $(90,88 ; 100,00)$ \\
\hline$\lambda_{011}$ & $\operatorname{Beta}(0,5 ; 0,5)$ & 50,00 & 35,30 & 88,72 & $(67,95 ; 99,92)$ \\
\hline$\lambda_{101}$ & $\operatorname{Beta}(0,5 ; 0,5)$ & 50,00 & 35,30 & 68,56 & $(57,85 ; 81,95)$ \\
\hline$\lambda_{001}$ & $\operatorname{Beta}(0,5 ; 0,5)$ & 50,00 & 35,30 & 2,98 & $(2,22 ; 3,84)$ \\
\hline$\lambda_{110}$ & $\operatorname{Beta}(0,5 ; 0,5)$ & 50,00 & 35,30 & 84,40 & $(69,04 ; 95,38)$ \\
\hline$\lambda_{010}$ & $\operatorname{Beta}(0,5 ; 0,5)$ & 50,00 & 35,30 & 93,54 & $(74,65 ; 99,99)$ \\
\hline$\lambda_{100}$ & $\operatorname{Beta}(0,5 ; 0,5)$ & 50,00 & 35,30 & 92,96 & $(72,54 ; 99,99)$ \\
\hline$\lambda_{000}$ & $\operatorname{Beta}(0,5 ; 0,5)$ & 50,00 & 35,30 & 95,35 & $(78,64 ; 100,00)$ \\
\hline
\end{tabular}

(a) desvio padrão

Na Tabela 61 foram consideradas distribuições a priori, Beta $(0,5 ; 0,5)$, não informativas para todos os parâmetros $\lambda_{i j d}(i=0,1 ; j=0,1 ; d=0,1)$, e informativas para $S_{E 1}$, $S_{E 2}, S_{P 1}, S_{P 2}$ e $\xi$

Tabela 61: Resultados a posteriori para os dados de RATNAM et al. (2000).

\begin{tabular}{|c|c|c|c|c|c|}
\hline \multirow[b]{2}{*}{ Parâmetro } & \multicolumn{3}{|c|}{ Informações a priori } & \multicolumn{2}{|c|}{ Informações a posteriori } \\
\hline & Distribuições & Média (\%) & $\mathrm{DP}^{(a)}(\%)$ & Média (\%) & ICr $95 \%$ \\
\hline$\xi$ & $\operatorname{Beta}(2,75 ; 8,25)$ & 25,00 & 12,50 & 8,86 & $(7,49 ; 10,72)$ \\
\hline$S_{E 1}$ & $\operatorname{Beta}(1,85 ; 7,40)$ & 20,00 & 12,50 & 58,91 & $(49,35 ; 67,00)$ \\
\hline$S_{E 2}$ & $\operatorname{Beta}(1,85 ; 7,40)$ & 20,00 & 12,50 & 28,19 & $(21,34 ; 35,30)$ \\
\hline$S_{P 1}$ & $\operatorname{Beta}(7,40 ; 1,85)$ & 80,00 & 12,50 & 93,91 & $(92,76 ; 95,04)$ \\
\hline$S_{P 2}$ & $\operatorname{Beta}(7,40 ; 1,85)$ & 80,00 & 12,50 & 97,85 & $(97,15 ; 98,46)$ \\
\hline$\lambda_{111}$ & $\operatorname{Beta}(0,5 ; 0,5)$ & 50,00 & 35,30 & 88,74 & $(77,06 ; 96,63)$ \\
\hline$\lambda_{011}$ & $\operatorname{Beta}(0,5 ; 0,5)$ & 50,00 & 35,30 & 92,89 & $(72,83 ; 99,99)$ \\
\hline$\lambda_{101}$ & $\operatorname{Beta}(0,5 ; 0,5)$ & 50,00 & 35,30 & 95,73 & $(81,42 ; 100,00)$ \\
\hline$\lambda_{001}$ & $\operatorname{Beta}(0,5 ; 0,5)$ & 50,00 & 35,30 & 88,02 & $(56,80 ; 99,98)$ \\
\hline$\lambda_{110}$ & $\operatorname{Beta}(0,5 ; 0,5)$ & 50,00 & 35,30 & 97,55 & $(88,12 ; 100,00)$ \\
\hline$\lambda_{010}$ & $\operatorname{Beta}(0,5 ; 0,5)$ & 50,00 & 35,30 & 89,53 & $(69,83 ; 99,92)$ \\
\hline$\lambda_{100}$ & $\operatorname{Beta}(0,5 ; 0,5)$ & 50,00 & 35,30 & 63,07 & $(52,20 ; 75,52)$ \\
\hline$\lambda_{000}$ & $\operatorname{Beta}(0,5 ; 0,5)$ & 50,00 & 35,30 & 8,04 & $(6,84 ; 9,35)$ \\
\hline
\end{tabular}

(a) desvio padrão

Na Tabela 62 foram consideradas distribuições a priori informativas $S_{E 1}, S_{E 2}, S_{P 1}$, $S_{P 2}, \xi$ e $\lambda_{i j d}(i=0,1 ; j=0,1 ; d=0,1)$. 
Tabela 62: Resultados a posteriori para os dados de RATNAM et al. (2000).

\begin{tabular}{|c|c|c|c|c|c|}
\hline \multirow[b]{2}{*}{ Parâmetro } & \multicolumn{3}{|c|}{ Informações a priori } & \multicolumn{2}{|c|}{ Informações a posteriori } \\
\hline & Distribuições & Média (\%) & $\mathrm{DP}^{(a)}(\%)$ & Média (\%) & ICr $95 \%$ \\
\hline$\xi$ & $\operatorname{Beta}(2,75 ; 8,25)$ & 25,00 & 12,50 & 11,02 & $(8,99 ; 13,60)$ \\
\hline$S_{E 1}$ & $\operatorname{Beta}(1,85 ; 7,40)$ & 20,00 & 12,50 & 51,00 & $(40,93 ; 60,59)$ \\
\hline$S_{E 2}$ & $\operatorname{Beta}(1,85 ; 7,40)$ & 20,00 & 12,50 & 22,94 & $(16,83 ; 29,71)$ \\
\hline$S_{P 1}$ & $\operatorname{Beta}(7,40 ; 1,85)$ & 80,00 & 12,50 & 94,17 & $(92,91 ; 95,36)$ \\
\hline$S_{P 2}$ & $\operatorname{Beta}(7,40 ; 1,85)$ & 80,00 & 12,50 & 97,80 & $(97,09 ; 98,43)$ \\
\hline$\lambda_{111}$ & $\operatorname{Beta}(8,10 ; 2,00)$ & 80,00 & 12,50 & 87,93 & $(77,22 ; 95,67)$ \\
\hline$\lambda_{011}$ & $\operatorname{Beta}(2,6 ; 2,6)$ & 50,00 & 20,00 & 83,12 & $(63,41 ; 96,38)$ \\
\hline$\lambda_{101}$ & $\operatorname{Beta}(2,6 ; 2,6)$ & 50,00 & 20,00 & 85,12 & $(73,12 ; 96,51)$ \\
\hline$\lambda_{001}$ & $\operatorname{Beta}(2,00 ; 8,10)$ & 20,00 & 12,50 & 50,67 & $(32,52 ; 70,37)$ \\
\hline$\lambda_{110}$ & $\operatorname{Beta}(8,10 ; 2,00)$ & 80,00 & 12,50 & 93,34 & $(82,18 ; 99,14)$ \\
\hline$\lambda_{010}$ & $\operatorname{Beta}(2,6 ; 2,6)$ & 50,00 & 20,00 & 82,39 & $(63,05 ; 95,87)$ \\
\hline$\lambda_{100}$ & $\operatorname{Beta}(2,6 ; 2,6)$ & 50,00 & 20,00 & 68,01 & $(55,30 ; 82,34)$ \\
\hline$\lambda_{000}$ & $\operatorname{Beta}(2,00 ; 8,10)$ & 20,00 & 12,50 & 8,27 & $(7,01 ; 9,63)$ \\
\hline
\end{tabular}

a) desvio padrão

Na Tabela 63 foram consideradas distribuições a priori, Beta $(0,5 ; 0,5)$, não informativas para todos os parâmetros $\lambda_{i j d}(i=0,1 ; j=0,1 ; d=0,1)$ e $\xi$, e informativas para $S_{E 1}, S_{E 2}, S_{P 1}$ e $S_{P 2}$.

Tabela 63: Resultados a posteriori para os dados de RATNAM et al. (2000).

\begin{tabular}{|c|c|c|c|c|c|}
\hline \multirow[b]{2}{*}{ Parâmetro } & \multicolumn{3}{|c|}{ Informações a priori } & \multicolumn{2}{|c|}{ Informações a posteriori } \\
\hline & Distribuições & Média (\%) & $\mathrm{DP}^{(a)}(\%)$ & Média (\%) & ICr $95 \%$ \\
\hline$\xi$ & $\operatorname{Beta}(0,5 ; 0,5)$ & 50,00 & 35,30 & 8,76 & $(7,39 ; 10,54)$ \\
\hline$S_{E 1}$ & $\operatorname{Beta}(1,85 ; 7,40)$ & 20,00 & 12,50 & 59,09 & $(49,79 ; 67,08)$ \\
\hline$S_{E 2}$ & $\operatorname{Beta}(1,85 ; 7,40)$ & 20,00 & 12,50 & 28,35 & $(21,59 ; 35,61)$ \\
\hline$S_{P 1}$ & $\operatorname{Beta}(7,40 ; 1,85)$ & 80,00 & 12,50 & 93,90 & $(92,76 ; 95,01)$ \\
\hline$S_{P 2}$ & $\operatorname{Beta}(7,40 ; 1,85)$ & 80,00 & 12,50 & 97,85 & $(97,17 ; 98,45)$ \\
\hline$\lambda_{111}$ & $\operatorname{Beta}(0,5 ; 0,5)$ & 50,00 & 35,30 & 88,75 & $(76,90 ; 96,71)$ \\
\hline$\lambda_{011}$ & $\operatorname{Beta}(0,5 ; 0,5)$ & 50,00 & 35,30 & 92,96 & $(72,98 ; 99,99)$ \\
\hline$\lambda_{101}$ & $\operatorname{Beta}(0,5 ; 0,5)$ & 50,00 & 35,30 & 95,92 & $(81,94 ; 99,99)$ \\
\hline$\lambda_{001}$ & $\operatorname{Beta}(0,5 ; 0,5)$ & 50,00 & 35,30 & 88,87 & $(59,24 ; 99,99)$ \\
\hline$\lambda_{110}$ & $\operatorname{Beta}(0,5 ; 0,5)$ & 50,00 & 35,30 & 97,55 & $(87,97 ; 100,00)$ \\
\hline$\lambda_{010}$ & $\operatorname{Beta}(0,5 ; 0,5)$ & 50,00 & 35,30 & 89,37 & $(69,55 ; 99,91)$ \\
\hline$\lambda_{100}$ & $\operatorname{Beta}(0,5 ; 0,5)$ & 50,00 & 35,30 & 62,83 & $(51,94 ; 74,94)$ \\
\hline$\lambda_{000}$ & $\operatorname{Beta}(0,5 ; 0,5)$ & 50,00 & 35,30 & 8,03 & $(6,83 ; 9,33)$ \\
\hline
\end{tabular}

(a) desvio padrão

Na Tabela 64 foram consideradas distribuições a priori, Beta $(0,5 ; 0,5)$, não informativas para $S_{E 1}, S_{E 2}, S_{P 1}, S_{P 2}$ e $\xi$, e informativas para $\lambda_{i j d}(i=0,1 ; j=0,1 ; d=0$, $1)$. 
Tabela 64: Resultados a posteriori para os dados de RATNAM et al. (2000).

\begin{tabular}{|c|c|c|c|c|c|}
\hline \multirow[b]{2}{*}{ Parâmetro } & \multicolumn{3}{|c|}{ Informações a priori } & \multicolumn{2}{|c|}{ Informações a posteriori } \\
\hline & Distribuições & Média (\%) & $\mathrm{DP}^{(a)}(\%)$ & Média (\%) & ICr $95 \%$ \\
\hline$\xi$ & $\operatorname{Beta}(0,5 ; 0,5)$ & 50,00 & 35,30 & 10,52 & $(8,63 ; 12,85)$ \\
\hline$S_{E 1}$ & $\operatorname{Beta}(0,5 ; 0,5)$ & 50,00 & 35,30 & 54,72 & $(44,71 ; 64,24)$ \\
\hline$S_{E 2}$ & $\operatorname{Beta}(0,5 ; 0,5)$ & 50,00 & 35,30 & 24,11 & $(17,84 ; 31,01)$ \\
\hline$S_{P 1}$ & $\operatorname{Beta}(0,5 ; 0,5)$ & 50,00 & 35,30 & 94,28 & $(93,02 ; 95,47)$ \\
\hline$S_{P 2}$ & $\operatorname{Beta}(0,5 ; 0,5)$ & 50,00 & 35,30 & 97,88 & $(97,16 ; 98,48)$ \\
\hline$\lambda_{111}$ & $\operatorname{Beta}(8,10 ; 2,00)$ & 80,00 & 12,50 & 87,90 & $(77,27 ; 95,62)$ \\
\hline$\lambda_{011}$ & $\operatorname{Beta}(2,6 ; 2,6)$ & 50,00 & 20,00 & 83,12 & $(63,33 ; 96,38)$ \\
\hline$\lambda_{101}$ & $\operatorname{Beta}(2,6 ; 2,6)$ & 50,00 & 20,00 & 84,62 & $(69,80 ; 96,32$ \\
\hline$\lambda_{001}$ & $\operatorname{Beta}(2,00 ; 8,10)$ & 20,00 & 12,50 & 55,77 & $(36,93 ; 75,03)$ \\
\hline$\lambda_{110}$ & $\operatorname{Beta}(8,10 ; 2,00)$ & 80,00 & 12,50 & 93,44 & $(82,51 ; 99,21)$ \\
\hline$\lambda_{010}$ & $\operatorname{Beta}(2,6 ; 2,6)$ & 50,00 & 20,00 & 82,29 & $(63,04 ; 95,66)$ \\
\hline$\lambda_{100}$ & $\operatorname{Beta}(2,6 ; 2,6)$ & 50,00 & 20,00 & 68,44 & $(55,53 ; 83,55)$ \\
\hline$\lambda_{000}$ & $\operatorname{Beta}(2,00 ; 8,10)$ & 20,00 & 12,50 & 8,21 & $(6,98 ; 9,53)$ \\
\hline
\end{tabular}

(a) desvio padrão

Observando que dentre os 2098 indivíduos amostrados, 408 foram verificados, foram especificadas as probabilidades de verificação, iguais e constantes, dadas por $\lambda_{111}=\lambda_{101}=$ $\lambda_{011}=\lambda_{001}=\lambda_{110}=\lambda_{100}=\lambda_{010}=\lambda_{000}=408 / 2098=19,4 \%$. Foram consideradas distribuições a priori não informativas para $S_{E 1}, S_{E 2}, S_{P 1}, S_{P 2}$ e $\xi$. Os resultados estão na Tabela 65

Tabela 65: Resultados a posteriori para os dados de RATNAM et al. (2000).

\begin{tabular}{cllllll}
\hline & \multicolumn{2}{l}{ Informações $a$ priori } & & \multicolumn{2}{l}{ Informações $a$ posteriori } \\
\cline { 2 - 4 } \cline { 7 - 7 } Parâmetro & Distribuições & Média $(\%)$ & DP $^{(a)}(\%)$ & & Média $(\%)$ & ICr $95 \%$ \\
\hline$\xi$ & Beta $(0,5 ; 0,5)$ & 50,00 & 35,30 & & 23,28 & $(18,71 ; 28,50)$ \\
$S_{E 1}$ & Beta $(0,5 ; 0,5)$ & 50,00 & 35,30 & & 26,61 & $(20,52 ; 33,61)$ \\
$S_{E 2}$ & Beta $(0,5 ; 0,5)$ & 50,00 & 35,30 & & 10,91 & $(7,76 ; 14,71)$ \\
$S_{P 1}$ & Beta $(0,5 ; 0,5)$ & 50,00 & 35,30 & & 93,86 & $(92,49 ; 95,08)$ \\
$S_{P 2}$ & Beta $(0,5 ; 0,5)$ & 50,00 & 35,30 & & 97,50 & $(96,66 ; 98,23)$ \\
$\lambda_{i j d}^{(b)}$ & constante & 19,40 & - & & - & - \\
\hline
\end{tabular}

${ }^{(a)}$ desvio padrão; ${ }^{(b)} i=0,1 ; j=0,1 ; d=0,1$.

Na Tabela 66 foram consideradas distribuições a priori não informativas para $S_{E 1}$, $S_{E 2}, S_{P 1}, S_{P 2}$ e $\xi$, e ainda, valores constantes para $\lambda_{i j d}(i=0,1 ; j=0,1 ; d=0,1)$. 
Tabela 66: Resultados a posteriori para os dados de RATNAM et al. (2000).

\begin{tabular}{|c|c|c|c|c|c|}
\hline \multirow[b]{2}{*}{ Parâmetro } & \multicolumn{3}{|c|}{ Informações a priori } & \multicolumn{2}{|c|}{ Informações a posteriori } \\
\hline & Distribuições & Média (\%) & $\operatorname{DP}^{(a)}(\%)$ & Média (\%) & ICr $95 \%$ \\
\hline$\xi$ & $\operatorname{Beta}(0,5 ; 0,5)$ & 50,00 & 35,30 & 23,52 & $(19,07 ; 28,74)$ \\
\hline$S_{E 1}$ & $\operatorname{Beta}(1,85 ; 7,40)$ & 20,00 & 12,50 & 26,13 & $(20,30 ; 32,80)$ \\
\hline$S_{E 2}$ & $\operatorname{Beta}(1,85 ; 7,40)$ & 20,00 & 12,50 & 10,88 & $(7,80 ; 14,60)$ \\
\hline$S_{P 1}$ & $\operatorname{Beta}(7,40 ; 1,85)$ & 80,00 & 12,50 & 93,77 & $(92,42 ; 94,97)$ \\
\hline$S_{P 2}$ & $\operatorname{Beta}(7,40 ; 1,85)$ & 80,00 & 12,50 & 97,43 & $(96,59 ; 98,16)$ \\
\hline$\lambda_{i j d}^{(b)}$ & constante & 19,40 & - & - & - \\
\hline
\end{tabular}

Nota-se, a partir dos resultados obtidos, que, para os dados dos dois exemplos (HALL et al., 1996 e RATNAM et al., 2000), a especificação das informações a priori são de extrema importância para o modelo, já que, quando estas não são informativas o suficiente, levam à estimação insatisfatória dos parâmetros de interesse, quando comparadas às estimativas de máxima verossimilhança. Assim, faz-se necessário o bom conhecimento e informação do profissional da área médica, sobre o teste que este utiliza, para que a metodologia leve a resultados que possam ser de utilidade para saúde pública.

A Tabela 67, a seguir, mostra as freqüências de dados simulados (completos) quando se cruza os resultados de dois testes diagnósticos $\left(T_{1}\right.$ e $\left.T_{2}\right)$ com um padrão ouro $(D)$. Observa-se uma amostra de tamanho $n=500$, em que existem 224 portadores da doença $(D=1)$ e 276 não portadores $(D=0)$. Considerando os dados completos, para o teste 1 $\left(T_{1}\right)$, tem-se uma sensibilidade estimada em $(83+71) / 224=68,8 \%$ e uma especificidade de $(39+140) / 276=64,9 \%$. Já para o teste $2\left(T_{2}\right)$, o valor da sensibilidade é estimado por $(83+17) / 224=44,6 \%$ e a especificidade é igual a $(59+140) / 276=72,1 \%$. A prevalência é estimada em $224 / 500=44,8 \%$.

Tabela 67: Freqüência do cruzamento entre $D$ e $T$ - dados completos.

\begin{tabular}{ccclcc}
\hline & \multicolumn{2}{c}{$T_{1}=1$} & & \multicolumn{2}{c}{$T_{1}=0$} \\
\cline { 2 - 3 } \cline { 5 - 6 } & $T_{2}=1$ & $T_{2}=0$ & & $T_{2}=1$ & $T_{2}=0$ \\
\hline$D=1$ & 83 & 71 & & 17 & 53 \\
$D=0$ & 38 & 59 & & 39 & 140 \\
Total & 121 & 130 & & 56 & 193 \\
\hline
\end{tabular}


A partir dos dados exibidos na Tabela 67 , suponha-se que $\lambda_{111}=\lambda_{101}=\lambda_{011}=$ $\lambda_{001}=\lambda_{110}=\lambda_{100}=\lambda_{010}=\lambda_{000}=79,8 \%$, o que significa que está-se assumindo que a probabilidade de verificação independe dos resultados dos testes $\left(T_{1}\right.$ e $\left.T_{2}\right)$ e verdadeiro estado do indivíduo $(D)$. Os dados obtidos encontram-se na Tabela 68.

Tabela 68: Freqüência do cruzamento entre $D, T$ e $V$ - dados simulados.

\begin{tabular}{cccccc}
\hline & \multicolumn{2}{c}{$T_{1}=1$} & & \multicolumn{2}{c}{$T_{1}=0$} \\
\cline { 2 - 3 } \cline { 5 - 6 } & $T_{2}=1$ & $T_{2}=0$ & & $T_{2}=1$ & $T_{2}=0$ \\
\hline$V=1, D=1$ & 66 & 57 & & 14 & 42 \\
$V=1, D=0$ & 30 & 47 & & 31 & 112 \\
$V=0$ & 25 & 26 & & 11 & 39 \\
Total & 121 & 130 & & 56 & 193 \\
\hline
\end{tabular}

Assim, as estimativas de máxima verossimilhança, bem como seus intervalos de confiança $95 \%$, propostas por Zhou (1998), para essa situação, são dadas por $\widehat{S_{E 1}}=68,8 \%$ $(62,3 \% ; 75,3 \%), \widehat{S_{E 2}}=44,8 \%(37,8 \% ; 51,8 \%), \widehat{S_{P 1}}=65,0 \%(59,0 \% ; 70,9 \%)$ e $\widehat{S_{P 2}}=$ $72,3 \%(66,7 \% ; 77,9 \%)$. A prevalência é estimada em $\widehat{\xi}=44,9 \%$. Nota-se que essas estimativas são muito próximas daquelas obtidas a partir do banco de dados completo (Tabela 67). Considerando essa situação (indivíduos verificados e não verificados), algumas simulações foram feitas, afim de se verificar a eficiência da metodologia bayesiana, introduzida, na estimação das medidas de desempenho para dois testes diagnósticos, na presença do viés de verificação. Agora, tem-se o verdadeiro valor dessas medidas, obtidas a partir do banco de dados completo, para se comparar com os valores estimados. Inicialmente, na Tabela 69, foram consideradas distribuições a priori não informativas para todos os parâmetros. Utilizando o software WinBugs, versão 1.4, foram geradas 205 mil amostras, das quais as primeiras 5000 foram descartadas para que as inferências estivessem livres dos efeitos dos valores iniciais. Também foram consideradas apenas as $15^{a}, 30^{a}, 45^{a}, \ldots$ iterações, resultando em, aproximadamente, 13 mil amostras de Gibbs. A convergência do algoritmo foi verificada a partir de gráficos temporais das amostras geradas e utilizando-se técnicas usuais existentes na literatura (GELMAN e RUBIN, 1992). 
Tabela 69: Resultados a posteriori - dados simulados - distribuições a priori não informativas.

\begin{tabular}{|c|c|c|c|c|c|}
\hline \multirow[b]{2}{*}{ Parâmetro } & \multicolumn{3}{|c|}{ Informações a priori } & \multicolumn{2}{|c|}{ Informações a posteriori } \\
\hline & Distribuições & Média (\%) & $\mathrm{DP}^{(a)}(\%)$ & Média $(\%)$ & ICr $95 \%$ \\
\hline$\xi$ & $\operatorname{Beta}(0,5 ; 0,5)$ & 50,00 & 35,30 & 44,94 & $(39,05 ; 51,04)$ \\
\hline$S_{E 1}$ & $\operatorname{Beta}(0,5 ; 0,5)$ & 50,00 & 35,30 & 70,31 & $(63,00 ; 77,13)$ \\
\hline$S_{E 2}$ & $\operatorname{Beta}(0,5 ; 0,5)$ & 50,00 & 35,30 & 49,59 & $(41,67 ; 57,41)$ \\
\hline$S_{P 1}$ & $\operatorname{Beta}(0,5 ; 0,5)$ & 50,00 & 35,30 & 66,30 & $(59,12 ; 73,47)$ \\
\hline$S_{P 2}$ & $\operatorname{Beta}(0,5 ; 0,5)$ & 50,00 & 35,30 & 76,01 & $(69,87 ; 81,42)$ \\
\hline$\lambda_{111}$ & $\operatorname{Beta}(0,5 ; 0,5)$ & 50,00 & 35,30 & 74,00 & $(63,59 ; 85,16)$ \\
\hline$\lambda_{011}$ & $\operatorname{Beta}(0,5 ; 0,5)$ & 50,00 & 35,30 & 63,50 & $(39,84 ; 95,67)$ \\
\hline$\lambda_{101}$ & $\operatorname{Beta}(0,5 ; 0,5)$ & 50,00 & 35,30 & 83,30 & $(63,25 ; 99,89)$ \\
\hline$\lambda_{001}$ & $\operatorname{Beta}(0,5 ; 0,5)$ & 50,00 & 35,30 & 94,46 & $(74,26 ; 99,99)$ \\
\hline$\lambda_{110}$ & $\operatorname{Beta}(0,5 ; 0,5)$ & 50,00 & 35,30 & 92,99 & $(68,97 ; 99,99)$ \\
\hline$\lambda_{010}$ & $\operatorname{Beta}(0,5 ; 0,5)$ & 50,00 & 35,30 & 91,39 & $(70,18 ; 99,99)$ \\
\hline$\lambda_{100}$ & $\operatorname{Beta}(0,5 ; 0,5)$ & 50,00 & 35,30 & 78,32 & $(57,73 ; 99,79)$ \\
\hline$\lambda_{000}$ & $\operatorname{Beta}(0,5 ; 0,5)$ & 50,00 & 35,30 & 75,16 & $(67,34 ; 83,03)$ \\
\hline
\end{tabular}

Observando que dentre os 500 indivíduos amostrados, 399 foram verificados, foram especificadas as probabilidades de verificação, iguais e constantes, dadas por $\lambda_{111}=\lambda_{101}=$ $\lambda_{011}=\lambda_{001}=\lambda_{110}=\lambda_{100}=\lambda_{010}=\lambda_{000}=399 / 500=79,8 \%$. Foram consideradas distribuições a priori não informativas para $S_{E 1}, S_{E 2}, S_{P 1}, S_{P 2}$ e $\xi$. Os resultados estão na Tabela 70.

Tabela 70: Resultados a posteriori - dados simulados - distribuições a priori não informativas.

\begin{tabular}{|c|c|c|c|c|c|}
\hline \multirow[b]{2}{*}{ Parâmetro } & \multicolumn{3}{|c|}{ Informações a priori } & \multicolumn{2}{|c|}{ Informações a posteriori } \\
\hline & Distribuições & Média (\%) & $\mathrm{DP}^{(a)}(\%)$ & Média (\%) & ICr $95 \%$ \\
\hline$\xi$ & $\operatorname{Beta}(0,5 ; 0,5)$ & 50,00 & 35,30 & 44,94 & $(40,22 ; 49,8)$ \\
\hline$S_{E 1}$ & $\operatorname{Beta}(0,5 ; 0,5)$ & 50,00 & 35,30 & 69,16 & $(62,57 ; 75,30)$ \\
\hline$S_{E 2}$ & $\operatorname{Beta}(0,5 ; 0,5)$ & 50,00 & 35,30 & 45,73 & $(39,04 ; 52,62)$ \\
\hline$S_{P 1}$ & $\operatorname{Beta}(0,5 ; 0,5)$ & 50,00 & 35,30 & 65,29 & $(59,25 ; 71,12)$ \\
\hline$S_{P 2}$ & $\operatorname{Beta}(0,5 ; 0,5)$ & 50,00 & 35,30 & 72,94 & $(67,38 ; 78,22)$ \\
\hline$\lambda_{i j d}^{(b)}$ & constante & 79,80 & - & - & - \\
\hline
\end{tabular}

${ }^{(a)}$ desvio padrão; ${ }^{(b)} i=0,1 ; j=0,1 ; d=0,1$.

Na Tabela 71 foram consideradas distribuições a priori não informativas para todos os parâmetros, mas foram fixados os resultados para o verdadeiro estado da doença $(D)$, reduzindo-se o número de parâmetros relacionados à verificação $\left(\lambda_{i j d}\right)$. 
Tabela 71: Resultados a posteriori - dados simulados - distribuições a priori não informativas.

\begin{tabular}{|c|c|c|c|c|c|}
\hline \multirow[b]{2}{*}{ Parâmetro } & \multicolumn{3}{|c|}{ Informações a priori } & \multicolumn{2}{|c|}{ Informações a posteriori } \\
\hline & Distribuições & Média (\%) & $\mathrm{DP}^{(a)}(\%)$ & Média (\%) & ICr $95 \%$ \\
\hline$\xi$ & $\operatorname{Beta}(0,5 ; 0,5)$ & 50,00 & 35,30 & 45,12 & $(38,46 ; 51,98)$ \\
\hline$S_{E 1}$ & $\operatorname{Beta}(0,5 ; 0,5)$ & 50,00 & 35,30 & 68,84 & $(62,06 ; 75,23)$ \\
\hline$S_{E 2}$ & $\operatorname{Beta}(0,5 ; 0,5)$ & 50,00 & 35,30 & 45,54 & $(38,73 ; 52,52)$ \\
\hline$S_{P 1}$ & $\operatorname{Beta}(0,5 ; 0,5)$ & 50,00 & 35,30 & 65,13 & $(58,91 ; 71,14)$ \\
\hline$S_{P 2}$ & $\operatorname{Beta}(0,5 ; 0,5)$ & 50,00 & 35,30 & 72,83 & $(67,04 ; 78,19)$ \\
\hline$\lambda_{i j 1}^{(b)}$ & $\operatorname{Beta}(0,5 ; 0,5)$ & 50,00 & 35,30 & 79,48 & $(69,26 ; 90,65)$ \\
\hline$\lambda_{i j 0}^{(b)}$ & $\operatorname{Beta}(0,5 ; 0,5)$ & 50,00 & 35,30 & 80,27 & $(71,60 ; 89,58)$ \\
\hline
\end{tabular}

${ }^{(a)}$ desvio padrão; ${ }^{(b)} i=0,1 ; j=0,1$.

Na Tabela 72 foram consideradas distribuições a priori não informativas para todos os parâmetros, mas fixaram-se os resultados para o teste $1\left(T_{1}\right)$, reduzindo-se o número de parâmetros relacionados à verificação $\left(\lambda_{i j d}\right)$.

Tabela 72: Resultados a posteriori - dados simulados - distribuições a priori não informativas.

\begin{tabular}{cllllll}
\hline & \multicolumn{2}{l}{ Informações a priori } & & \multicolumn{2}{l}{ Informações a posteriori } \\
\cline { 2 - 3 } Parâmetro & Distribuições & Média $(\%)$ & $\operatorname{DP}^{(a)}(\%)$ & & Média $(\%)$ & ICr $95 \%$ \\
\hline$\xi$ & Beta $(0,5 ; 0,5)$ & 50,00 & 35,30 & & 44,94 & $(40,14 ; 49,74)$ \\
$S_{E 1}$ & Beta $(0,5 ; 0,5)$ & 50,00 & 35,30 & & 69,19 & $(62,55 ; 75,40)$ \\
$S_{E 2}$ & Beta $(0,5 ; 0,5)$ & 50,00 & 35,30 & & 45,73 & $(38,80 ; 52,71)$ \\
$S_{P 1}$ & Beta $(0,5 ; 0,5)$ & 50,00 & 35,30 & & 65,30 & $(59,40 ; 71,08)$ \\
$S_{P 2}$ & Beta $(0,5 ; 0,5)$ & 50,00 & 35,30 & & 72,92 & $(67,24 ; 78,28)$ \\
$\lambda_{1 j d}^{(b)}$ & Beta $(0,5 ; 0,5)$ & 50,00 & 35,30 & & 79,58 & $(74,39 ; 84,29)$ \\
$\lambda_{0 j d}^{(b)}$ & Beta $(0,5 ; 0,5)$ & 50,00 & 35,30 & & 79,83 & $(74,62 ; 84,60)$ \\
\hline
\end{tabular}

${ }^{(a)}$ desvio padrão; ${ }^{(b)} j=0,1 ; d=0,1$.

Na Tabela 73 foram consideradas distribuições a priori não informativas para todos os parâmetros, mas fixaram-se os resultados para o teste $2\left(T_{2}\right)$, reduzindo-se o número de parâmetros relacionados à verificação $\left(\lambda_{i j d}\right)$. 
Tabela 73: Resultados a posteriori - dados simulados - distribuições a priori não informativas.

\begin{tabular}{|c|c|c|c|c|c|}
\hline \multirow[b]{2}{*}{ Parâmetro } & \multicolumn{3}{|c|}{ Informações a priori } & \multicolumn{2}{|c|}{ Informações a posteriori } \\
\hline & Distribuições & Média (\%) & $\mathrm{DP}^{(a)}(\%)$ & Média (\%) & ICr $95 \%$ \\
\hline$\xi$ & $\operatorname{Beta}(0,5 ; 0,5)$ & 50,00 & 35,30 & 44,89 & $(40,11 ; 49,76)$ \\
\hline$S_{E 1}$ & $\operatorname{Beta}(0,5 ; 0,5)$ & 50,00 & 35,30 & 69,13 & $(62,40 ; 75,39)$ \\
\hline$S_{E 2}$ & $\operatorname{Beta}(0,5 ; 0,5)$ & 50,00 & 35,30 & 45,67 & $(38,81 ; 52,66)$ \\
\hline$S_{P 1}$ & $\operatorname{Beta}(0,5 ; 0,5)$ & 50,00 & 35,30 & 65,30 & $(59,29 ; 71,20)$ \\
\hline$S_{P 2}$ & $\operatorname{Beta}(0,5 ; 0,5)$ & 50,00 & 35,30 & 72,92 & $(67,25 ; 78,33)$ \\
\hline$\lambda_{i 1 d}^{(b)}$ & $\operatorname{Beta}(0,5 ; 0,5)$ & 50,00 & 35,30 & 79,51 & $(73,27 ; 85,12)$ \\
\hline$\lambda_{i 0 d}^{(b)}$ & $\operatorname{Beta}(0,5 ; 0,5)$ & 50,00 & 35,30 & 79,78 & $(75,22 ; 83,93)$ \\
\hline
\end{tabular}

${ }^{(a)}$ desvio padrão; ${ }^{(b)} i=0,1 ; d=0,1$.

Na Tabela 74, foram consideradas distribuições a priori informativas, baseadas nas estimativas obtidas do banco de dados completo, para as medidas de desempenho de testes diagnósticos. Também foram consideradas distribuições a priori informativas para $\lambda_{i j d}(i=0,1 ; j=0,1 ; d=0,1)$, levando-se em conta que a probabilidade de se verificar um indivíduo que fornece um resultado posivito para um ou para os dois testes, seja maior do que para aquele que apresenta resultado negativo. Por outro lado, a probabilidade de se verificar um indivíduo que fornece resultados negativos para os dois testes deve ser pequena.

Tabela 74: Resultados a posteriori - dados simulados - distribuições a priori informativas.

\begin{tabular}{|c|c|c|c|c|c|}
\hline \multirow[b]{2}{*}{ Parâmetro } & \multicolumn{3}{|c|}{ Informações a priori } & \multicolumn{2}{|c|}{ Informações a posteriori } \\
\hline & Distribuições & Média (\%) & $\mathrm{DP}^{(a)}(\%)$ & Média (\%) & ICr $95 \%$ \\
\hline$\xi$ & $\operatorname{Beta}(6,7 ; 8,1)$ & 45,00 & 12,50 & 45,86 & $(40,38 ; 51,35)$ \\
\hline$S_{E 1}$ & $\operatorname{Beta}(8,7 ; 3,7)$ & 70,00 & 12,50 & 68,09 & $(61,31 ; 74,68)$ \\
\hline$S_{E 2}$ & $\operatorname{Beta}(6,7 ; 8,1)$ & 45,00 & 12,50 & 47,80 & $(40,61 ; 54,95)$ \\
\hline$S_{P 1}$ & $\operatorname{Beta}(8,8 ; 4,8)$ & 65,00 & 12,50 & 63,81 & $(57,14 ; 70,25)$ \\
\hline$S_{P 2}$ & $\operatorname{Beta}(8,7 ; 3,7)$ & 70,00 & 12,50 & 74,10 & $(68,02 ; 79,57)$ \\
\hline$\lambda_{111}$ & $\operatorname{Bet} a(8,10 ; 2,00)$ & 80,00 & 12,50 & 76,91 & $(66,64 ; 87,80)$ \\
\hline$\lambda_{011}$ & $\operatorname{Bet} a(2,6 ; 2,6)$ & 50,00 & 20,00 & 64,26 & $(42,60 ; 85,93)$ \\
\hline$\lambda_{101}$ & $\operatorname{Beta}(2,6 ; 2,6)$ & 50,00 & 20,00 & 80,17 & $(66,05 ; 93,37)$ \\
\hline$\lambda_{001}$ & $\operatorname{Beta}(2,00 ; 8,10)$ & 20,00 & 12,50 & 70,61 & $(55,18 ; 84,45)$ \\
\hline$\lambda_{110}$ & $\operatorname{Bet} a(8,10 ; 2,00)$ & 80,00 & 12,50 & 85,84 & $(67,09 ; 97,88)$ \\
\hline$\lambda_{010}$ & $\operatorname{Beta}(2,6 ; 2,6)$ & 50,00 & 20,00 & 83,63 & $(67,38 ; 95,90)$ \\
\hline$\lambda_{100}$ & $\operatorname{Beta}(2,6 ; 2,6)$ & 50,00 & 20,00 & 76,06 & $(60,61 ; 91,58)$ \\
\hline$\lambda_{000}$ & $\operatorname{Beta}(2,00 ; 8,10)$ & 20,00 & 12,50 & 75,78 & $(67,40 ; 83,97)$ \\
\hline
\end{tabular}

${ }^{(a)}$ desvio padrão

Na Tabela 75, foram consideradas distribuições a priori informativas, baseadas nas 
estimativas obtidas do banco de dados completo, para as medidas de desempenho de testes diagnósticos. Também foram consideradas distribuições a priori informativas para $\lambda_{i j d}(i=0,1 ; j=0,1 ; d=0,1)$, baseando-se no fato que foram retirados, e considerados não verificados, $20 \%$ dos indivíduos de cada célula da Tabela $67 ; \operatorname{logo}, \lambda_{i j d}=79,8 \%$.

Tabela 75: Resultados a posteriori - dados simulados - distribuições a priori informativas.

\begin{tabular}{|c|c|c|c|c|c|}
\hline \multirow[b]{2}{*}{ Parâmetro } & \multicolumn{3}{|c|}{ Informações a priori } & \multicolumn{2}{|c|}{ Informações a posteriori } \\
\hline & Distribuições & Média (\%) & $\mathrm{DP}^{(a)}(\%)$ & Média $(\%)$ & ICr $95 \%$ \\
\hline$\xi$ & $\operatorname{Beta}(6,7 ; 8,1)$ & 45,00 & 12,50 & 44,53 & $(39,20 ; 50,10)$ \\
\hline$S_{E 1}$ & $\operatorname{Beta}(8,7 ; 3,7)$ & 70,00 & 12,50 & 70,07 & $(63,17 ; 76,54)$ \\
\hline$S_{E 2}$ & $\operatorname{Beta}(6,7 ; 8,1)$ & 45,00 & 12,50 & 49,16 & $(41,85 ; 56,42)$ \\
\hline$S_{P 1}$ & $\operatorname{Beta}(8,8 ; 4,8)$ & 65,00 & 12,50 & 64,76 & $(58,27 ; 70,96)$ \\
\hline$S_{P 2}$ & $\operatorname{Beta}(8,7 ; 3,7)$ & 70,00 & 12,50 & 74,61 & $(68,69 ; 79,99)$ \\
\hline$\lambda_{111}$ & $\operatorname{Beta}(8,10 ; 2,00)$ & 80,00 & 12,50 & 76,60 & $(66,54 ; 86,75)$ \\
\hline$\lambda_{011}$ & $\operatorname{Beta}(8,10 ; 2,00)$ & 80,00 & 12,50 & 73,48 & $(53,01 ; 92,70)$ \\
\hline$\lambda_{101}$ & $\operatorname{Bet} a(8,10 ; 2,00)$ & 80,00 & 12,50 & 82,79 & $(68,01 ; 96,23)$ \\
\hline$\lambda_{001}$ & $\operatorname{Beta}(8,10 ; 2,00)$ & 80,00 & 12,50 & 89,08 & $(72,88 ; 98,52)$ \\
\hline$\lambda_{110}$ & $\operatorname{Beta}(8,10 ; 2,00)$ & 80,00 & 12,50 & 86,65 & $(68,10 ; 98,03)$ \\
\hline$\lambda_{010}$ & $\operatorname{Beta}(8,10 ; 2,00)$ & 80,00 & 12,50 & 85,51 & $(70,25 ; 97,18)$ \\
\hline$\lambda_{100}$ & $\operatorname{Beta}(8,10 ; 2,00)$ & 80,00 & 12,50 & 78,13 & $(62,70 ; 94,11)$ \\
\hline$\lambda_{000}$ & $\operatorname{Beta}(8,10 ; 2,00)$ & 80,00 & 12,50 & 76,67 & $(69,12 ; 84,29)$ \\
\hline
\end{tabular}

Observando que dentre os 500 indivíduos amostrados, 399 foram verificados, foram especificadas as probabilidades de verificação, iguais e constantes, dadas por $\lambda_{111}=\lambda_{101}=$ $\lambda_{011}=\lambda_{001}=\lambda_{110}=\lambda_{100}=\lambda_{010}=\lambda_{000}=399 / 500=79,8 \%$. Foram consideradas distribuições a priori informativas para $S_{E 1}, S_{E 2}, S_{P 1}, S_{P 2}$ e $\xi$. Os resultados estão na Tabela 76.

Tabela 76: Resultados a posteriori - dados simulados - distribuições a priori informativas.

\begin{tabular}{|c|c|c|c|c|c|}
\hline \multirow[b]{2}{*}{ Parâmetro } & \multicolumn{3}{|c|}{ Informações a priori } & \multicolumn{2}{|c|}{ Informações a posteriori } \\
\hline & Distribuições & Média (\%) & $\mathrm{DP}^{(a)}(\%)$ & Média (\%) & ICr $95 \%$ \\
\hline$\xi$ & $\operatorname{Beta}(6,7 ; 8,1)$ & 45,00 & 12,50 & 44,87 & $(40,18 ; 49,66)$ \\
\hline$S_{E 1}$ & $\operatorname{Beta}(8,7 ; 3,7)$ & 70,00 & 12,50 & 69,24 & $(62,90 ; 75,30)$ \\
\hline$S_{E 2}$ & $\operatorname{Beta}(6,7 ; 8,1)$ & 45,00 & 12,50 & 46,85 & $(40,32 ; 53,59)$ \\
\hline$S_{P 1}$ & $\operatorname{Beta}(8,8 ; 4,8)$ & 65,00 & 12,50 & 64,28 & $(58,42 ; 69,93)$ \\
\hline$S_{P 2}$ & $\operatorname{Beta}(8,7 ; 3,7)$ & 70,00 & 12,50 & 72,91 & $(67,27 ; 78,21)$ \\
\hline$\lambda_{i j d}^{(b)}$ & constante & 79,80 & - & - & - \\
\hline
\end{tabular}

${ }^{(a)}$ desvio padrão; ${ }^{(b)} i=0,1 ; j=0,1 ; d=0,1$. 
Nas Tabelas 77 e 78 foram consideradas distribuições a priori informativas para todos os parâmetros, mas foram fixados os resultados para o verdadeiro estado da doença $(D)$, reduzindo-se o número de parâmetros relacionados à verificação $\left(\lambda_{i j d}\right)$.

Tabela 77: Resultados a posteriori - dados simulados - distribuições a priori informativas.

\begin{tabular}{|c|c|c|c|c|c|}
\hline \multirow[b]{2}{*}{ Parâmetro } & \multicolumn{3}{|c|}{ Informações a priori } & \multicolumn{2}{|c|}{ Informações a posteriori } \\
\hline & Distribuições & Média (\%) & $\mathrm{DP}^{(a)}(\%)$ & Média $(\%)$ & ICr $95 \%$ \\
\hline$\xi$ & $\operatorname{Beta}(6,7 ; 8,1)$ & 45,00 & 12,50 & 42,52 & $(36,80 ; 48,38)$ \\
\hline$S_{E 1}$ & $\operatorname{Beta}(8,7 ; 3,7)$ & 70,00 & 12,50 & 69,57 & $(63,13 ; 75,70)$ \\
\hline$S_{E 2}$ & $\operatorname{Beta}(6,7 ; 8,1)$ & 45,00 & 12,50 & 47,10 & $(40,31 ; 54,06)$ \\
\hline$S_{P 1}$ & $\operatorname{Beta}(8,8 ; 4,8)$ & 65,00 & 12,50 & 63,18 & $(57,19 ; 69,15)$ \\
\hline$S_{P 2}$ & $\operatorname{Beta}(8,7 ; 3,7)$ & 70,00 & 12,50 & 72,32 & $(66,73 ; 77,53)$ \\
\hline$\lambda_{i j 1}^{(b)}$ & $\operatorname{Beta}(8,10 ; 2,00)$ & 80,00 & 12,50 & 84,33 & $(75,23 ; 93,63)$ \\
\hline$\lambda_{i j 0}^{(b)}$ & $\operatorname{Beta}(2,00 ; 8,10)$ & 20,00 & 12,50 & 74,60 & $(67,35 ; 81,77)$ \\
\hline
\end{tabular}

Tabela 78: Resultados a posteriori - dados simulados - distribuições a priori informativas.

\begin{tabular}{|c|c|c|c|c|c|}
\hline \multirow[b]{2}{*}{ Parâmetro } & \multicolumn{3}{|c|}{ Informações a priori } & \multicolumn{2}{|c|}{ Informações a posteriori } \\
\hline & Distribuições & Média (\%) & $\mathrm{DP}^{(a)}(\%)$ & Média $(\%)$ & ICr $95 \%$ \\
\hline$\xi$ & $\operatorname{Beta}(6,7 ; 8,1)$ & 45,00 & 12,50 & 43,17 & $(38,13 ; 49,34)$ \\
\hline$S_{E 1}$ & $\operatorname{Beta}(8,7 ; 3,7)$ & 70,00 & 12,50 & 69,13 & $(62,56 ; 75,44)$ \\
\hline$S_{E 2}$ & $\operatorname{Beta}(6,7 ; 8,1)$ & 45,00 & 12,50 & 46,79 & $(40,13 ; 53,38)$ \\
\hline$S_{P 1}$ & $\operatorname{Beta}(8,8 ; 4,8)$ & 65,00 & 12,50 & 64,05 & $(58,07 ; 69,92)$ \\
\hline$S_{P 2}$ & $\operatorname{Beta}(8,7 ; 3,7)$ & 70,00 & 12,50 & 72,83 & $(67,30 ; 78,08)$ \\
\hline$\lambda_{i j 1}^{(b)}$ & $\operatorname{Beta}(8,10 ; 2,00)$ & 80,00 & 12,50 & 80,24 & $(70,84 ; 89,57)$ \\
\hline$\lambda_{i j 0}^{(b)}$ & $\operatorname{Beta}(8,10 ; 2,00)$ & 80,00 & 12,50 & 79,79 & $(72,06 ; 87,76)$ \\
\hline
\end{tabular}

${ }^{(a)}$ desvio padrão; ${ }^{(b)} i=0,1 ; j=0,1$.

Nas Tabelas 79 e 80 foram consideradas distribuições a priori não informativas para todos os parâmetros, mas fixaram-se os resultados para o teste $1\left(T_{1}\right)$, reduzindo-se o número de parâmetros relacionados à verificação $\left(\lambda_{i j d}\right)$.

Nas Tabelas 81 e 82 foram consideradas distribuições a priori não informativas para todos os parâmetros, mas fixaram-se os resultados para o teste $2\left(T_{2}\right)$, reduzindo-se o número de parâmetros relacionados à verificação $\left(\lambda_{i j d}\right)$. 
Tabela 79: Resultados a posteriori - dados simulados - distribuições a priori informativas.

\begin{tabular}{|c|c|c|c|c|c|}
\hline \multirow[b]{2}{*}{ Parâmetro } & \multicolumn{3}{|c|}{ Informações a priori } & \multicolumn{2}{|c|}{ Informações a posteriori } \\
\hline & Distribuições & Média (\%) & $\mathrm{DP}^{(a)}(\%)$ & Média (\%) & ICr $95 \%$ \\
\hline$\xi$ & $\operatorname{Beta}(6,7 ; 8,1)$ & 45,00 & 12,50 & 44,86 & $(40,17 ; 49,57)$ \\
\hline$S_{E 1}$ & $\operatorname{Beta}(8,7 ; 3,7)$ & 70,00 & 12,50 & 69,28 & $(62,84 ; 75,35)$ \\
\hline$S_{E 2}$ & $\operatorname{Beta}(6,7 ; 8,1)$ & 45,00 & 12,50 & 46,91 & $(40,25 ; 53,47)$ \\
\hline$S_{P 1}$ & $\operatorname{Beta}(8,8 ; 4,8)$ & 65,00 & 12,50 & 64,23 & $(58,42 ; 69,79)$ \\
\hline$S_{P 2}$ & $\operatorname{Beta}(8,7 ; 3,7)$ & 70,00 & 12,50 & 72,98 & $(67,34 ; 78,15)$ \\
\hline$\lambda_{1 j d}^{(b)}$ & $\operatorname{Beta}(8,10 ; 2,00)$ & 80,00 & 12,50 & 79,70 & $(74,66 ; 84,33)$ \\
\hline$\lambda_{0 j d}^{(b)}$ & $\operatorname{Beta}(2,00 ; 8,10)$ & 20,00 & 12,50 & 77,58 & $(77,58 ; 82,45)$ \\
\hline
\end{tabular}

${ }^{(a)}$ desvio padrão; ${ }^{(b)} j=0,1 ; d=0,1$.

Tabela 80: Resultados a posteriori - dados simulados - distribuições a priori informativas.

\begin{tabular}{|c|c|c|c|c|c|}
\hline \multirow[b]{2}{*}{ Parâmetro } & \multicolumn{3}{|c|}{ Informações a priori } & \multicolumn{2}{|c|}{ Informações a posteriori } \\
\hline & Distribuições & Média (\%) & $\mathrm{DP}^{(a)}(\%)$ & Média (\%) & ICr $95 \%$ \\
\hline$\xi$ & $\operatorname{Beta}(6,7 ; 8,1)$ & 45,00 & 12,50 & 44,87 & $(40,18 ; 49,63)$ \\
\hline$S_{E 1}$ & $\operatorname{Beta}(8,7 ; 3,7)$ & 70,00 & 12,50 & 69,25 & $(63,01 ; 75,31)$ \\
\hline$S_{E 2}$ & $\operatorname{Beta}(6,7 ; 8,1)$ & 45,00 & 12,50 & 46,87 & $(40,31 ; 53,49)$ \\
\hline$S_{P 1}$ & $\operatorname{Beta}(8,8 ; 4,8)$ & 65,00 & 12,50 & 64,19 & $(58,37 ; 68,89)$ \\
\hline$S_{P 2}$ & $\operatorname{Beta}(8,7 ; 3,7)$ & 70,00 & 12,50 & 72,95 & $(67,28 ; 78,21)$ \\
\hline$\lambda_{1 j d}^{(b)}$ & $\operatorname{Beta}(8,10 ; 2,00)$ & 80,00 & 12,50 & 79,70 & $(74,67 ; 84,35)$ \\
\hline$\lambda_{0 j d}^{(b)}$ & $\operatorname{Beta}(8,10 ; 2,00)$ & 80,00 & 12,50 & 79,93 & $(74,76 ; 84,60)$ \\
\hline
\end{tabular}

${ }^{(a)}$ desvio padrão; ${ }^{(b)} j=0,1 ; d=0,1$.

Tabela 81: Resultados a posteriori - dados simulados - distribuições a priori informativas.

\begin{tabular}{|c|c|c|c|c|c|}
\hline \multirow[b]{2}{*}{ Parâmetro } & \multicolumn{3}{|c|}{ Informações a priori } & \multicolumn{2}{|c|}{ Informações a posteriori } \\
\hline & Distribuições & Média (\%) & $\mathrm{DP}^{(a)}(\%)$ & Média $(\%)$ & ICr $95 \%$ \\
\hline$\xi$ & $\operatorname{Beta}(6,7 ; 8,1)$ & 45,00 & 12,50 & 44,87 & $(40,18 ; 49,60)$ \\
\hline$S_{E 1}$ & $\operatorname{Beta}(8,7 ; 3,7)$ & 70,00 & 12,50 & 69,28 & $(62,82 ; 75,26)$ \\
\hline$S_{E 2}$ & $\operatorname{Beta}(6,7 ; 8,1)$ & 45,00 & 12,50 & 46,89 & $(40,15 ; 53,56)$ \\
\hline$S_{P 1}$ & $\operatorname{Beta}(8,8 ; 4,8)$ & 65,00 & 12,50 & 64,22 & $(58,35 ; 69,89)$ \\
\hline$S_{P 2}$ & $\operatorname{Beta}(8,7 ; 3,7)$ & 70,00 & 12,50 & 72,94 & $(67,48 ; 78,21)$ \\
\hline$\lambda_{i 1 d}^{(b)}$ & $\operatorname{Beta}(8,10 ; 2,00)$ & 80,00 & 12,50 & 79,73 & $(73,75 ; 85,17)$ \\
\hline$\lambda_{i 0 d}^{(b)}$ & $\operatorname{Beta}(2,00 ; 8,10)$ & 20,00 & 12,50 & 78,05 & $(73,33 ; 82,41)$ \\
\hline
\end{tabular}

${ }^{(a)}$ desvio padrão; ${ }^{(b)} i=0,1 ; d=0,1$. 
Tabela 82: Resultados a posteriori - dados simulados - distribuições a priori informativas.

\begin{tabular}{|c|c|c|c|c|c|}
\hline \multirow[b]{2}{*}{ Parâmetro } & \multicolumn{3}{|c|}{ Informações a priori } & \multicolumn{2}{|c|}{ Informações a posteriori } \\
\hline & Distribuições & Média (\%) & $\mathrm{DP}^{(a)}(\%)$ & Média (\%) & ICr $95 \%$ \\
\hline$\xi$ & $\operatorname{Beta}(6,7 ; 8,1)$ & 45,00 & 12,50 & 44,82 & $(40,13 ; 49,62)$ \\
\hline$S_{E 1}$ & $\operatorname{Beta}(8,7 ; 3,7)$ & 70,00 & 12,50 & 69,26 & $(62,91 ; 75,28)$ \\
\hline$S_{E 2}$ & $\operatorname{Beta}(6,7 ; 8,1)$ & 45,00 & 12,50 & 46,91 & $(40,36 ; 53,58)$ \\
\hline$S_{P 1}$ & $\operatorname{Beta}(8,8 ; 4,8)$ & 65,00 & 12,50 & 64,25 & $(58,40 ; 69,98)$ \\
\hline$S_{P 2}$ & $\operatorname{Beta}(8,7 ; 3,7)$ & 70,00 & 12,50 & 72,92 & $(67,35 ; 78,16)$ \\
\hline$\lambda_{i 1 d}^{(b)}$ & $\operatorname{Bet} a(8,10 ; 2,00)$ & 80,00 & 12,50 & 79,67 & $(73,73 ; 85,07)$ \\
\hline$\lambda_{i 0 d}^{(b)}$ & $\operatorname{Beta}(8,10 ; 2,00)$ & 80,00 & 12,50 & 79,91 & $(75,37 ; 84,03)$ \\
\hline
\end{tabular}

\subsection{Método Bayesiano (testes dependentes)}

Considere-se, ainda, que o pressuposto de que $P\left(V \mid T_{1}, T_{2}, D\right)=P\left(V \mid T_{1}, T_{2}\right)$ é inválido, ou seja, a verificação depende do verdadeiro estado da doença. Admitindo-se que existe dependência entre os resultados de $T_{1}$ e $T_{2}$, a covariância entre $T_{1}$ e $T_{2}$, dado $D=1$, pode ser escrita por $\psi_{D}=\operatorname{cov}\left(T_{1}, T_{2} \mid D=1\right)=P\left(T_{1}, T_{2} \mid D=1\right)-P\left(T_{1} \mid D=\right.$ 1) $P\left(T_{2} \mid D=1\right)$. Portanto, $P\left(T_{1}=1, T_{2}=1, D=1\right)=P(D=1) P\left(T_{1}=1, T_{2}=1 \mid\right.$ $D=1)=\xi\left[S_{E 1} S_{E 2}+\psi_{D}\right]\left(\right.$ ver VACEK, 1985). Assim, $P\left(T_{1}=1, T_{2}=1, V=1, D=1\right)=$ $P\left(T_{1}=1, T_{2}=1, D=1\right) P\left(V=1 \mid T_{1}=1, T_{2}=1, D=1\right)=\xi \lambda_{111}\left[S_{E 1} S_{E 2}+\psi_{D}\right]$. Seja $\psi_{N D}$ a covariância entre os testes $T_{1}$ e $T_{2}$, dado $D=0$, então as possíveis combinações entre as variáveis $V, D, T_{1}$ e $T_{2}$ e as suas contribuições à verossimilhança são dadas na Tabela 83.

Nota-se, então, que $P\left(T_{1}=1, T_{2}=1\right)$ sofre um aumento de $\psi_{D}$ quando os dois testes são correlacionados, o que não acontece quando eles são independentes. Aqui, será assumido que $\psi_{D}$ e $\psi_{N D}$ são positivos, o que é mais freqüentemente observado na prática (DENDUKURI e JOSEPH, 2001).

$\mathrm{Na}$ análise bayesiana assume-se que as densidades a priori para todos os parâmetros do vetor $\boldsymbol{\theta}=\left(\xi, S_{E 1}, S_{E 2}, S_{P 1}, S_{P 2}, \lambda_{111}, \lambda_{101}, \lambda_{011}, \lambda_{001}, \lambda_{110}, \lambda_{100}, \lambda_{010}, \lambda_{000}\right)$ possuem distribuição possuem distribuição Beta, em que $\alpha_{\theta}$ e $\beta_{\theta}$ são hiperparâmetros (conhecidos) da distribuição de $\boldsymbol{\theta}$.

Os parâmetros relacionados às covariâncias entre os testes $\left(\psi_{D}\right.$ e $\left.\psi_{N D}\right)$ possuem 
Tabela 83: Contribuições de todas as possíveis combinações entre as variáveis $V, D, T_{1}$ e $T_{2}$ à verossimilhança.

\begin{tabular}{cccccc}
\hline$T_{1}$ & $T_{2}$ & $D$ & $V$ & Freqüência & Contribuição à Verossimilhança \\
\hline 1 & 1 & 1 & 1 & $s_{11}$ & $\xi \lambda_{111}\left[S_{E 1} S_{E 2}+\psi_{D}\right]$ \\
1 & 0 & 1 & 1 & $s_{10}$ & $\xi \lambda_{101}\left[S_{E 1}\left(1-S_{E 2}\right)-\psi_{D}\right]$ \\
0 & 1 & 1 & 1 & $s_{01}$ & $\xi \lambda_{011}\left[\left(1-S_{E 1}\right) S_{E 2}-\psi_{D}\right]$ \\
0 & 0 & 1 & 1 & $s_{00}$ & $\xi \lambda_{001}\left[\left(1-S_{E 1}\right)\left(1-S_{E 2}\right)+\psi_{D}\right]$ \\
1 & 1 & 0 & 1 & $r_{11}$ & $(1-\xi) \lambda_{110}\left[\left(1-S_{P 1}\right)\left(1-S_{P 2}\right)+\psi_{N D}\right]$ \\
1 & 0 & 0 & 1 & $r_{10}$ & $(1-\xi) \lambda_{100}\left[\left(1-S_{P 1}\right) S_{P 2}-\psi_{N D}\right]$ \\
0 & 1 & 0 & 1 & $r_{01}$ & $(1-\xi) \lambda_{010}\left[S_{P 1}\left(1-S_{P 2}\right)-\psi_{N D}\right]$ \\
0 & 0 & 0 & 1 & $r_{00}$ & $(1-\xi) \lambda_{000}\left[S_{P 1} S_{P 2}+\psi_{N D}\right]$ \\
1 & 1 & 1 & 0 & {$\left[u_{111}\right]$} & $\xi\left(1-\lambda_{111}\right)\left[S_{P 1} S_{P 2}+\psi_{D}\right]$ \\
1 & 0 & 1 & 0 & {$\left[u_{101}\right]$} & $\xi\left(1-\lambda_{101}\right)\left[S_{P 1}\left(1-S_{P 2}\right)-\psi_{D}\right]$ \\
0 & 1 & 1 & 0 & {$\left[u_{011}\right]$} & $\xi\left(1-\lambda_{011}\right)\left[\left(1-S_{P 1}\right) S_{P 2}-\psi_{D}\right]$ \\
0 & 0 & 1 & 0 & {$\left[u_{001}\right]$} & $\xi\left(1-\lambda_{001}\right)\left[\left(1-S_{P 1}\right)\left(1-S_{P 2}\right)+\psi_{D}\right]$ \\
1 & 1 & 0 & 0 & {$\left[u_{110}\right]$} & $(1-\xi)\left(1-\lambda_{110}\right)\left[\left(1-S_{P 1}\right)\left(1-S_{P 2}\right)+\psi_{N D}\right]$ \\
1 & 0 & 0 & 0 & {$\left[u_{100}\right]$} & $(1-\xi)\left(1-\lambda_{100}\right)\left[\left(1-S_{P 1}\right) S_{P 2}-\psi_{N D}\right]$ \\
0 & 1 & 0 & 0 & {$\left[u_{010}\right]$} & $(1-\xi)\left(1-\lambda_{010}\right)\left[S_{P 1}\left(1-S_{P 2}\right)-\psi_{N D}\right]$ \\
0 & 0 & 0 & 0 & {$\left[u_{000}\right]$} & $(1-\xi)\left(1-\lambda_{000}\right)\left[S_{P 1} S_{P 2}+\psi_{N D}\right]$ \\
\hline
\end{tabular}

densidades a priori dadas por uma distribuição beta generalizada, denotada aqui por GBeta. Então, $\psi_{D} \sim \operatorname{GBeta}\left(\alpha_{\psi_{D}}, \beta_{\psi_{D}}\right) \operatorname{com} 0 \leq \psi_{D} \leq \min \left(S_{E 1}, S_{E 2}\right)-S_{E 1} S_{E 2}$ e $\psi_{N D} \sim \operatorname{GBeta}\left(\alpha_{\psi_{N D}}, \beta_{\psi_{N D}}\right) \operatorname{com} 0 \leq \psi_{N D} \leq \min \left(S_{P 1}, S_{P 2}\right)-S_{P 1} S_{P 2}$, em que $\min (a, b)$ é o mínimo entre $a$ e $b$, e $\alpha_{\psi_{D}}, \beta_{\psi_{D}}, \alpha_{\psi_{N D}}, \beta_{\psi_{N D}}$ são hiperparâmetros conhecidos de uma distribuição beta generalizada, que é uma beta padrão, mas com uma mudança na escala, diferente do intervalo [0, 1] (DENDUKURI e JOSEPH, 2001).

Considerando-se os dados simulados, dados na Tabela 84, simulações foram feitas para se avaliar a influência dos parâmetros de dependência entre os testes $T_{1}$ e $T_{2}$, na estimação das medidas de desempenho dos mesmos.

Tabela 84: Freqüência do cruzamento entre $D, T$ e $V$ - dados simulados.

\begin{tabular}{cccccc}
\hline & \multicolumn{2}{c}{$T_{1}=1$} & & \multicolumn{2}{c}{$T_{1}=0$} \\
\cline { 2 - 3 } \cline { 5 - 6 } & $T_{2}=1$ & $T_{2}=0$ & & $T_{2}=1$ & $T_{2}=0$ \\
\hline$V=1, D=1$ & 66 & 57 & & 14 & 42 \\
$V=1, D=0$ & 30 & 47 & & 31 & 112 \\
$V=0$ & 25 & 26 & & 11 & 39 \\
Total & 121 & 130 & & 56 & 193 \\
\hline
\end{tabular}

A partir dos dados completos (ver Tabela 67 ), para o teste $1\left(T_{1}\right)$, tem-se uma sensibilidade estimada em $(83+71) / 224=68,8 \%$ e uma especificidade de $(39+140) / 276=64,9 \%$. Já para o teste $2\left(T_{2}\right)$, o valor da sensibilidade é estimado por $(83+17) / 224=44,6 \%$ 
e a especificidade é igual a $(59+140) / 276=72,1 \%$. A prevalência é estimada em $224 / 500=44,8 \%$. Já as estimativas de máxima verossimilhança, propostas por Zhou (1998), para essa situação, são dadas por $\widehat{S_{E 1}}=68,8 \%, \widehat{S_{E 2}}=65,0 \%, \widehat{S_{P 1}}=44,8 \%$ e $\widehat{S_{P 2}}=72,3 \%$. A prevalência é estimada em $\widehat{\xi}=44,9 \%$.

\subsubsection{Simulações}

Utilizando o software WinBugs, versão 1.4, foram geradas 205 mil amostras, das quais as primeiras 5000 foram descartadas para que as inferências estivessem livres dos efeitos dos valores iniciais. Também foram consideradas apenas as $15^{a}, 30^{a}, 45^{a}, \ldots$ iterações, resultando em, aproximadamente, 13 mil amostras de Gibbs. A convergência do algoritmo foi verificada a partir de gráficos temporais das amostras geradas e utilizando-se técnicas usuais existentes na literatura (GELMAN e RUBIN, 1992). Inicialmente, foram consideradas distribuições a priori, Beta $(0,5 ; 0,5)$, não informativas para todos os parâmetros do vetor $\boldsymbol{\theta}$. Os resultados são dados por:

Tabela 85: Resultados a posteriori - dados simulados - distribuições a priori não informativas - testes dependentes.

\begin{tabular}{|c|c|c|c|c|c|}
\hline \multirow[b]{2}{*}{ Parâmetro } & \multicolumn{3}{|c|}{ Informações a priori } & \multicolumn{2}{|c|}{ Informações a posteriori } \\
\hline & Distribuições & Média $(\%)$ & $\mathrm{DP}^{(a)}(\%)$ & Média (\%) & ICr $95 \%$ \\
\hline$\xi$ & $\operatorname{Beta}(0,5 ; 0,5)$ & 50,00 & 35,30 & 45,69 & $(37,13 ; 54,70)$ \\
\hline$S_{E 1}$ & $\operatorname{Beta}(0,5 ; 0,5)$ & 50,00 & 35,30 & 65,70 & $(54,87 ; 75,92)$ \\
\hline$S_{E 2}$ & $\operatorname{Beta}(0,5 ; 0,5)$ & 50,00 & 35,30 & 43,46 & $(33,78 ; 53,50)$ \\
\hline$S_{P 1}$ & $\operatorname{Beta}(0,5 ; 0,5)$ & 50,00 & 35,30 & 62,54 & $(53,41 ; 71,68)$ \\
\hline$S_{P 2}$ & $\operatorname{Beta}(0,5 ; 0,5)$ & 50,00 & 35,30 & 71,27 & $(62,99 ; 78,96)$ \\
\hline$\lambda_{111}$ & $\operatorname{Beta}(0,5 ; 0,5)$ & 50,00 & 35,30 & 83,52 & $(65,97 ; 99,92)$ \\
\hline$\lambda_{011}$ & $\operatorname{Beta}(0,5 ; 0,5)$ & 50,00 & 35,30 & 74,47 & $(42,64 ; 99,88)$ \\
\hline$\lambda_{101}$ & $\operatorname{Beta}(0,5 ; 0,5)$ & 50,00 & 35,30 & 82,77 & $(62,75 ; 99,92)$ \\
\hline$\lambda_{001}$ & $\operatorname{Beta}(0,5 ; 0,5)$ & 50,00 & 35,30 & 73,98 & $(45,67 ; 99,92)$ \\
\hline$\lambda_{110}$ & $\operatorname{Beta}(0,5 ; 0,5)$ & 50,00 & 35,30 & 75,57 & $(47,16 ; 99,99)$ \\
\hline$\lambda_{010}$ & $\operatorname{Beta}(0,5 ; 0,5)$ & 50,00 & 35,30 & 84,36 & $(63,21 ; 99,95)$ \\
\hline$\lambda_{100}$ & $\operatorname{Beta}(0,5 ; 0,5)$ & 50,00 & 35,30 & 79,39 & $(57,27 ; 99,91)$ \\
\hline$\lambda_{000}$ & $\operatorname{Beta}(0,5 ; 0,5)$ & 50,00 & 35,30 & 84,61 & $(69,55 ; 99,92)$ \\
\hline
\end{tabular}

${ }^{(a)}$ desvio padrão

Na Tabela 86 encontram-se os resultados obtidos a partir de especificações a priori informativas, para todos os parâmetros do vetor $\boldsymbol{\theta}$. 
Tabela 86: Resultados a posteriori - dados simulados - distribuições a priori informativas - testes dependentes.

\begin{tabular}{|c|c|c|c|c|c|}
\hline \multirow[b]{2}{*}{ Parâmetro } & \multicolumn{3}{|c|}{ Informações a priori } & \multicolumn{2}{|c|}{ Informações a posteriori } \\
\hline & Distribuições & Média (\%) & $\mathrm{DP}^{(a)}(\%)$ & Média (\%) & ICr $95 \%$ \\
\hline$\xi$ & $\operatorname{Beta}(6,7 ; 8,1)$ & 45,00 & 12,50 & 45,67 & $(39,99 ; 51,42)$ \\
\hline$S_{E 1}$ & $\operatorname{Beta}(8,7 ; 3,7)$ & 70,00 & 12,50 & 66,78 & $(59,47 ; 73,72)$ \\
\hline$S_{E 2}$ & $\operatorname{Beta}(6,7 ; 8,1)$ & 45,00 & 12,50 & 45,60 & $(38,04 ; 53,16)$ \\
\hline$S_{P 1}$ & $\operatorname{Beta}(8,8 ; 4,8)$ & 65,00 & 12,50 & 62,31 & $(55,59 ; 69,01)$ \\
\hline$S_{P 2}$ & $\operatorname{Beta}(8,7 ; 3,7)$ & 70,00 & 12,50 & 72,08 & $(65,67 ; 78,08)$ \\
\hline$\lambda_{111}$ & $\operatorname{Bet} a(8,10 ; 2,00)$ & 80,00 & 12,50 & 80,22 & $(68,35 ; 93,91)$ \\
\hline$\lambda_{011}$ & $\operatorname{Beta}(2,6 ; 2,6)$ & 50,00 & 20,00 & 69,95 & $(45,81 ; 91,76)$ \\
\hline$\lambda_{101}$ & $\operatorname{Beta}(2,6 ; 2,6)$ & 50,00 & 20,00 & 80,99 & $(65,76 ; 94,86)$ \\
\hline$\lambda_{001}$ & $\operatorname{Beta}(2,00 ; 8,10)$ & 20,00 & 12,50 & 64,70 & $(49,65 ; 80,39)$ \\
\hline$\lambda_{110}$ & $\operatorname{Bet} a(8,10 ; 2,00)$ & 80,00 & 12,50 & 79,88 & $(58,77 ; 96,74)$ \\
\hline$\lambda_{010}$ & $\operatorname{Beta}(2,6 ; 2,6)$ & 50,00 & 20,00 & 79,65 & $(63,85 ; 94,29)$ \\
\hline$\lambda_{100}$ & $\operatorname{Beta}(2,6 ; 2,6)$ & 50,00 & 20,00 & 74,98 & $(59,33 ; 91,58)$ \\
\hline$\lambda_{000}$ & $\operatorname{Bet} a(2,00 ; 8,10)$ & 20,00 & 12,50 & 78,95 & $(69,92 ; 87,83)$ \\
\hline
\end{tabular}

Agora, reduzindo-se o número de parâmetros $\lambda_{i j d}$ de tal maneira que a verificação $(V)$ não seja independente do verdadeiro estado da doença $(D)$, tem-se que $\lambda_{111}=\lambda_{101}=$ $\lambda_{011}=\lambda_{001}=\lambda_{1}$ e $\lambda_{110}=\lambda_{100}=\lambda_{010}=\lambda_{000}=\lambda_{0}$. Na Tabela 87 encontram-se os resultados obtidos a partir de distribuições a priori não informativas para os parâmetros de interesse e na Tabela 88, foram consideradas distribuições a priori informativas.

Tabela 87: Resultados a posteriori - dados simulados - distribuições a priori nãoinformativas - testes dependentes.

\begin{tabular}{|c|c|c|c|c|c|}
\hline \multirow[b]{2}{*}{ Parâmetro } & \multicolumn{3}{|c|}{ Informações a priori } & \multicolumn{2}{|c|}{ Informações a posteriori } \\
\hline & Distribuições & Média (\%) & $\mathrm{DP}^{(a)}(\%)$ & Média (\%) & ICr $95 \%$ \\
\hline$\xi$ & $\operatorname{Beta}(0,5 ; 0,5)$ & 50,00 & 35,30 & 44,90 & $(40,13 ; 49,80)$ \\
\hline$S_{E 1}$ & $\operatorname{Beta}(0,5 ; 0,5)$ & 50,00 & 35,30 & 68,80 & $(62,11 ; 75,02)$ \\
\hline$S_{E 2}$ & $\operatorname{Beta}(0,5 ; 0,5)$ & 50,00 & 35,30 & 44,81 & $(38,06 ; 51,70)$ \\
\hline$S_{P 1}$ & $\operatorname{Beta}(0,5 ; 0,5)$ & 50,00 & 35,30 & 64,84 & $(58,71 ; 70,65)$ \\
\hline$S_{P 2}$ & $\operatorname{Beta}(0,5 ; 0,5)$ & 50,00 & 35,30 & 72,32 & $(66,54 ; 77,68)$ \\
\hline$\lambda_{1}$ & $\operatorname{Beta}(0,5 ; 0,5)$ & 50,00 & 35,30 & 79,62 & $(74,49 ; 84,38)$ \\
\hline$\lambda_{0}$ & $\operatorname{Beta}(0,5 ; 0,5)$ & 50,00 & 35,30 & 79,80 & $(74,53 ; 84,60)$ \\
\hline
\end{tabular}

${ }^{(a)}$ desvio padrão. 
Tabela 88: Resultados a posteriori - dados simulados - distribuições a priori informativas - testes dependentes.

\begin{tabular}{cllllll}
\hline \multirow{2}{*}{ Parâmetro } & \multicolumn{2}{l}{ Informações a priori } & & \multicolumn{2}{l}{ Informações a posteriori } \\
\cline { 2 - 3 } \cline { 7 - 7 } & Distribuições & Média $(\%)$ & $\mathrm{DP}^{(a)}(\%)$ & & Média $(\%)$ & ICr $95 \%$ \\
\hline$\xi$ & $\operatorname{Beta}(6,7 ; 8,1)$ & 45,00 & 12,50 & & 44,82 & $(40,13 ; 49,57)$ \\
$S_{E 1}$ & $\operatorname{Beta}(8,7 ; 3,7)$ & 70,00 & 12,50 & & 69,09 & $(62,72 ; 75,19)$ \\
$S_{E 2}$ & $\operatorname{Beta}(6,7 ; 8,1)$ & 45,00 & 12,50 & & 46,02 & $(39,33 ; 52,80)$ \\
$S_{P 1}$ & $\operatorname{Beta}(8,8 ; 4,8)$ & 65,00 & 12,50 & & 63,80 & $(57,98 ; 69,45)$ \\
$S_{P 2}$ & $\operatorname{Beta}(8,7 ; 3,7)$ & 70,00 & 12,50 & & 72,23 & $(66,69 ; 77,57)$ \\
$\lambda_{1}$ & $\operatorname{Beta}(8,10 ; 2,00)$ & 80,00 & 12,50 & & 79,68 & $(74,47,84,33)$ \\
$\lambda_{0}$ & $\operatorname{Beta}(2,00 ; 8,10)$ & 20,00 & 12,50 & & 77,61 & $(72,27 ; 82,44)$ \\
\hline
\end{tabular}

${ }^{(a)}$ desvio padrão.

A partir dos resultados observados nas simulações (dados simulados) e, considerandose independência e dependência entre $\left(T_{1}\right)$ e $\left(T_{2}\right)$, nota-se que a metodologia bayesiana mostrou-se um tanto robusta na estimação das medidas de desempenho dos testes diagnosticos, pois não houve grande ganho quando inseridos os parâmetros de correlação. Agora, levando-se em conta a precisão dessas medidas estimadas, ela forneceu resultados muito satisfatórios, quando comparados com os obtidos a partir do banco de dados completos. 


\section{Conclusão}

Este trabalho objetivou a avaliação da metodologia bayesiana na estimação da sensibilidade e da especificidade de testes diagnóstico na presença do viés de verificação. Inicialmente foi realizada uma revisão de literatura, apresentando alguns conceitos sobre o assunto e as metodologias propostas por alguns autores, tentando contornar esse problema, bem como suas limitações.

Após isso, foram realizadas simulações, a partir dos dados completos de três exemplo, obtidos a partir da literatura, para se verificar como a metodologia bayesiana se comportaria na estimação da sensibilidade e da especificidade. Notou-se que esta metodologia poderia ter um desempenho melhor, talvez, se a escolha das distribuições a priori fossem obtidas a partir de um especialista da área, já que as conseguidas a partir da própria amostra não forneceram resultados diferentes daqueles alcançados a partir das distribuições $a$ priori não informativas.

Também foi introduzida uma metodologia bayesiana para a estimação das medidas de desempenho de dois testes diagnósticos (dependentes ou independentes), quando o viés de verificação se faz presente e esta se mostrou eficiente, quando comparados os seus resultados com os obtidos através do método de máxima verossimilhança, se uma informação precisa, a priori, for fornecida pelo pesquisador da área médica. Já quando utilizados dados simulados, a estimação, os resultados foram satisfatórios, quando comparados com os obtidos a partir do banco de dados completo, mostrando-se robusta, no que diz respeito à inserção de parâmetros de correlação entre os testes.

Assim, conclui-se que o problema da falta de informação, em alguns estudos, continua sendo um grande desafio para os profissionais da área estatística, que buscam, cada vez mais, formas de adaptar seus modelos aos problemas encontrados nas mais diversas áreas do conhecimento. 


\section{Referências Bibliográficas}

ACHCAR, J., MARTINEZ, E. e LOUZADA-NETO, F. Binary data in the presence of covariates and misclassifications: a bayesian approach. Brazilian Journal of Probability and Statistics, v.19, p.65-84, 2005.

AGRESTI, A. Categorical Data Analysis. $2^{a}$ ed., Nova Iorque:John Wiley and Sons, 2002, 734p.

ALTMAN, D. e BLAND, J. Diagnostic tests 1: sensitivity and specificity. British Medical Journal, v.308, p.1552, 1994.

ALTMAN, D. e BLAND, J. Diagnostic tests 2: predictive values. British Medical Journal, n.309, p.102, 1994.

ALTMAN, D. e BLAND, J. Diagnostic tests 3: receiver operating characteristic. British Medical Journal, v.309, p.188-188, 1994.

BEGG, C. Biases in the assessment of diagnostic tests. Statistics in Medicine, v.6, n.4, p.411-423, 1987.

BEGG, C. e GREENES, R. Assessments of diagnostics tests when disease verification is subject to selection bias. Biometrics, v.39, p.207-215, 1983.

BEGG, C. e Mc NEIL, B. Assessment of radiologic tests: control of bias and other design considerations. Radiology, v.167, p.565-569, 1988.

BELlER, G., SMITH, T., ABELMANN, W., HABER, E. e HOOD, W. Digitalis intoxication: a prospective clinical study with serum level correlations. The New England Journal of Medicine, v.284, p.989-997, 1971.

BOX, G. e TIAO, G. Bayesian Inference in Statistical Analysis. $2^{a}$ ed., Nova Iorque: Wiley- Interscience, 608p., 1992.

BRENNER, H. How independent are multiple independent diagnostic classifications? Statistics in Medicine, v. 15, p. 1377-1386, 1996. 
BROWNE, R. H. Using the sample range as a basis for calculating sample size in power calculations. The American Statistician, v.55, n.4, 293-298, 2001.

CECIL, M., KOSINSKI, A., JONES, M., TAYLO, A., ALAZRAKI, N., PETTIGREW, R. e WEINTRAUB, W. The importance of work-up (verification) bias correction in assessing the accuracy of spect thallium-201 testing for the diagnosis of coronary artery disease. Journal of Clinical Epidemiology, v.49, n.7, p.735-742, 1996.

DENDUKURI, N. e JOSEPH, L. Bayesian approaches to modeling the conditional dependence between multiple diagnostic tests. Biometrics, v.57, n.1, p. 158-167, 2001.

DONALD, A. e VAN TIL, L. Evaluating screening tests for dementia and cognitive impairment in a heterogeneous population in the presence of verification bias. International Psychogeriatrics, v.13, n.1, p.203-214, 2001.

DRUM, D. e CHRISTACAPOULOS, J. Hepatic scientigraphy in clinical decision making. Journal of Nuclear Medicine, v.13, p.908-915, 1972.

FEINSTEIN, A. Misguided efforts and future challenges for research on diagnostic tests. Journal of Epidemiology and Community Health, v.56, p.330-332, 2002.

FLEISS, J., LEVIN, B. e PAIK, M. Statistical Methods for Rates and Proportions. $3^{a}$ ed., Nova Iorque: John Wiley and Sons, 2003, 760p.

FRANCO, L. e PASSOS, A. Fundamentos de Epidemiologia. $1^{a}$ ed., São Paulo: Editora Manole, 2005, 380p.

GELMAN, A. e RUBIN, B. D. Inference from iterative simulation using multiple sequences. Statistical Sciences, v.7, n.4, p.457-511, 1992.

GRINER, P., MAYEWSKI, R., MUSHLIN, A. e GREENLAND, P. Selection and interpretation of diagnostic tests and procedures. Annals of Internal Medicine, v.94, n.4, p.553- 600, 1981.

GUPTA, A. e ROEHRBORN, C. Verification and incorporation biases in studies assessing 
screening tests: Prostate-specific antigen as a example. Urology, v.64, p.106-111, 2004.

HALL, K. S., OGUNNIYI, A. O., HENDRICE, H. C., OSUNTOKUN, B. O., HUI, S. L., MUSICK, B., RODENBERG, C. S., UNVERZAGT, F. W., GUERJE, O. e BAIYEWU, O. A cross-cultural community based study of dementias: methods and performance of survey instrument. International Journal of Methods in Psychiatric Research, v.6, p.129-142, 1996.

HAND, D. Screening vs prevalence estimation. Applied Statistics, v.36, p.1-7, 1987.

HANLEY, J. A., DENDUKURI, N. e BEGG, C. Multiple imputation for correcting verification bias - letter to the editor. Statistics in Medicine, in press, 2007.

HANSEN, J. H., CAUDILL, S. e BOONE, J. Crisis in drug testing. The Journal of the American Medical Association, n.253, p.2382-2385, 1985.

HAREL, O. e ZHOU, X. Multiple imputation for correcting verification bias. Statistics in Medicine, v.25, p.3769-3786, 2006.

HAREL, O. e ZHOU, X. H. Rejoinder to multiple imputation for correcting verification bias - rejoinder. Statistics in Medicine, in press, 2007.

HIGGINS, M. e KELLER, J. Seven measures of ventilatory lung function: Population values and comparisson of their ability to discriminate between persons with and without chronic respiratory symptoms and disease, Tecumseh, Michigan. The American Review Respiratory Disease, v.108, p.258-272, 1973.

JOSEPH, L., GYORKOS, T. e COUPAL, L. Bayesian estimation of disease prevalence and the parameters of diagnostic tests in the absence of a gold standard. American Journal of Epidemiology, v.141, n.3, p.263-272, 1995.

KOSINSKI, A. e BARNHART, H. Accounting for nonignorable verification bias in assessment of diagnostic tests. Biometrics, v.59, p.163-171, 2003.

MARTINEZ, E., ACHCAR, J. e LOUZADA-NETO, F. Bayesian estimation of diagnostic tests accuracy for semi-latent data with covariates. Journal of Biopharmaceutical 
Statistics, v. 15, p.809-821, 2005.

MARTINEZ, E., ACHCAR, J. e LOUZADA-NETO, F. Estimators of sensitivity and specificity in the presence of verification bias: a bayesian approach. Computational Statistics and Data Analysis, v.51, p.601-611, 2006.

MASUR, J. e MONTEIRO, M. Validation of the CAGE alcoholism test in brazilian psichiatric inpatient hospital setting. Brazilian Journal of Medical and Biological Research, v.16, p.215-218, 1983.

MEDRONHO, R. A. Epidemiologia. $2^{a}$ ed., Rio de Janeiro: Editora Atheneu, 2004, $517 \mathrm{p}$.

MOL, B., LIJMER, J., VAN DER MEUlEN, J., PAJKRT, E., BILARDO, C. e BOSSUYT, P. Effect of study design on the association between nuchal translucency measurement and down syndrome. British Medical Journal, v.94, n.5, p.864-869, 1999.

NOFUENTES, J. A. R. e DEL CASTILLO, J. Comparing the likelihood ratios of two binary diagnostic tests in the presence of partial verification. Biometrical Journal, v.47, n.4, p.42-57, 2005.

PUnGliA, R., D’AMicO, A., CATALONA, W., ROEHL, K. e KUNTZ, K. Effect of verification bias on screening for prostate cancer by measurement of prostate-specific antigen. The New England Journal of Medicine, v.349, n.4, p.335-442, 2003.

RATNAM, S., FRANCO, E. L. e FERENCZY, A. Human papillomavirus testing for primary screening of cervical cancer precursors. Cancer Epidemiology Biomarkers and Prevention, v.9, p.945-951, 2000.

ROGER, V., PELlikKA, P., BELl, M., CHOW, C., BAIley, K. e SEWARD, J. Sex and test verification bias impact on the diagnostic value of exercise echocardiography. Circulation, v.95, n.2, p.405-410, 1997.

SANTOS, A., DERCHAIn, S., CALVERT, E., MARTINS, M., DUFlOTH, R. e MARTINEZ, E. Z. Performance of colpocytology with different observer review and hybrid capture II in the diagnosis of cervical intraepithelial neoplasia grade 2 and 3. Cadernos 
de Saúde Pública, v.19, p.1029-1037, 2003.

SELVIN, S. Statistical Analysis of Epidemiologic Data, $2^{a}$ ed., Nova Iorque: Oxford University Press, 1996, 467p.

SIMEL, D. L., SAMSA, G., e MATCHAR, D. Likelihood ratios with confidence: sample size estimation for diagnostic test studies. Journal of Clinical Epidemiology, v.44, p.763-770, 1991.

SMITH, A. F. M. e ROBERTS, G. Bayesian computation via the Gibbs sampler and related Markov chain Monte Carlo methods (with discussion). Journal of the Royal Statistical Society B, v.55, p.3-23, 1993.

SOX, H. C. Probability theory in the use of diagnostic test: an introduction to critical study of the literature. Annals of Internal Medicine, v.104, n.1, p.60-66, 1986.

SPIEGElHALTER, D. J., THOMAS, A., BEST, N. e LUNN, D. Win BUGS User Manual Version 1.4, Cambridge: MRC Biostatistics Unit, 2001, não paginado.

TANNER, M. A. e WONG, W. H. The calculation of posterior distributions by data augumentation. Journal of the American Statistical Association, v.82, n.398, p.528-540, 1987.

TORRANCE - RYNARD, V. L. e WALTER, S. D. Effects of dependent errors in the assessment of diagnostic test performance. Statistics in Medicine, v. 16, p. 2157-2175, 1997.

VACEK, P. M. The effect of conditional dependence on the evaluation of diagnostic tests. Biometrics, v. 41, n. 4, p. 959-968, 1985.

WEINER, D., RYAN, T., e MCCABE, C. Exercise stress testing correlations among history of angina, st-segment response and prevalence of coronary artery disease in the coronary artery surgery study (CASS). The New England Journal of Medicine, v.301, p.230-235, 1979.

YERUSHALMY, J. Statistical problems of medical diagnosis with special reference to 
x-ray techniques. Public Health Report, v.62, p.1432-1449, 1947.

ZHENG, Y., BARLOW, W. e CUTTER, G. Assessing accuracy of mammography in the presence of verification bias and intrareader correlation. Biometrics, v.61, p.259-268, 2005.

ZHOU, X. Comparing accuracies of two screening tests in a two-phase study for dementia. Applied Statistics, v.47, n.1, p.135-147, 1998.

ZHOU, X. Maximum likelihood estimators of sensitivity and specificity corrected for verification bias. Communications in Statistics: Theory and Methods, v.22, p.3177-98, 1993. 
Apêndices

\section{A Gráficos de autocorrelações}

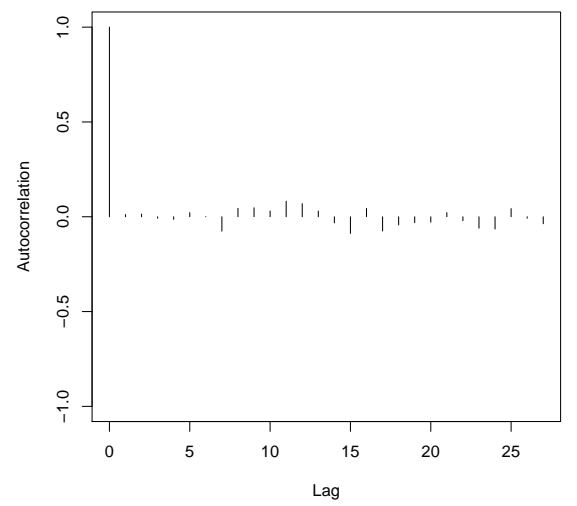

(a) sensibilidade

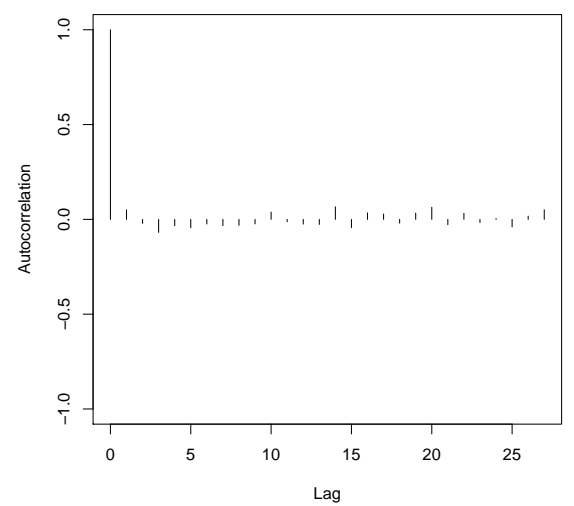

(c) sensibilidade

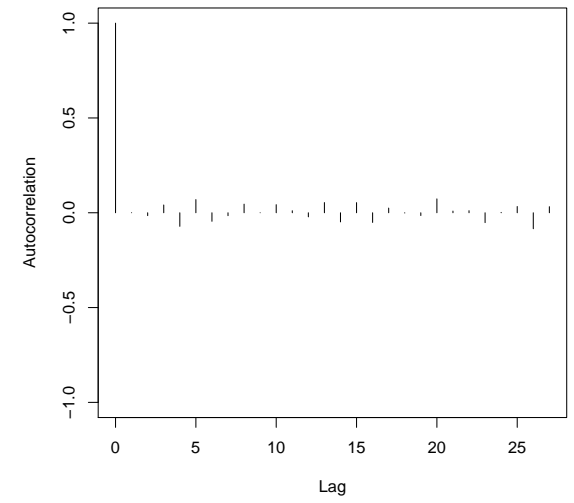

(b) especificidade

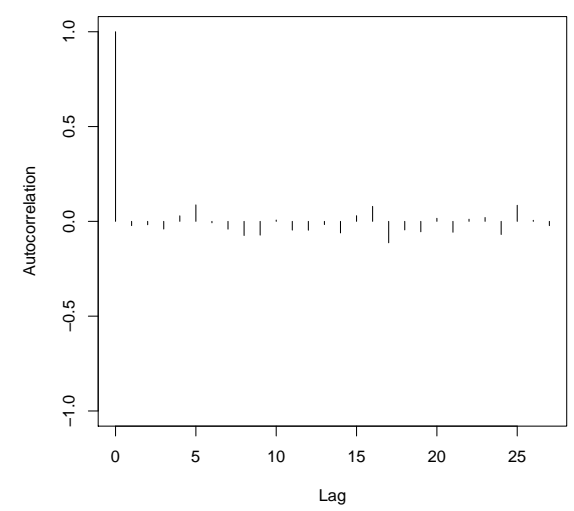

(d) especificidade

Figura 2: Gráficos de autocorrelações 


\section{B Gráficos de convergência}

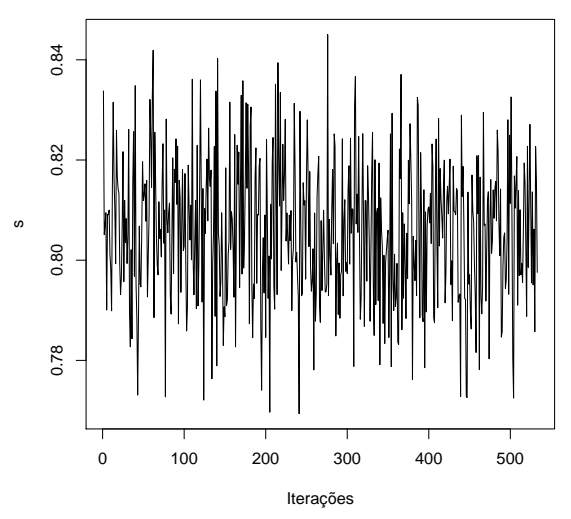

(a) sensibilidade

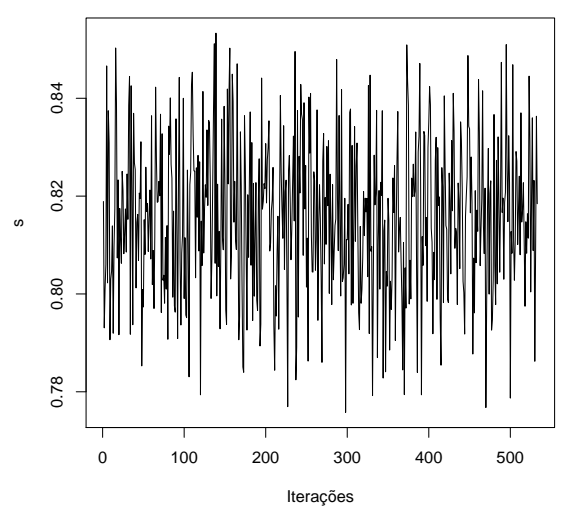

(c) sensibilidade

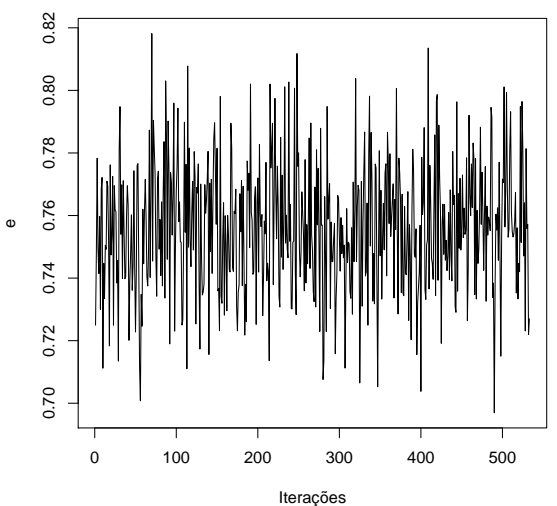

(b) especificidade

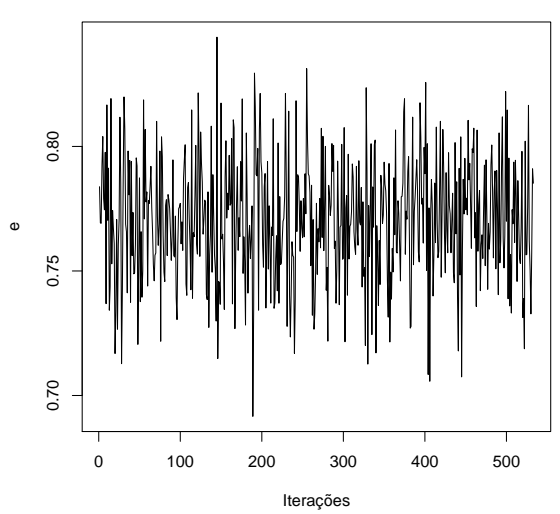

(d) especificidade

Figura 3: Gráficos de convergência 